\title{
SocialLab - Nutztierhaltung im Spiegel der Gesellschaft
}

Published online: 5 February 2018

(C) The Author(s) 2018. This article is an open access publication

\section{Erste Ergebnisse und Implikationen}

Inken Christoph-Schulz, Monika Hartmann ${ }^{2}$, Peter Kenning $^{3}$, Jörg Luy ${ }^{4}$, Marcus Mergenthaler ${ }^{5}$, Lucia Reisch $^{6}$, Jutta Roosen ${ }^{7}$, Achim Spiller ${ }^{8}$

${ }^{1}$ Thünen-Institut für Marktanalyse, Braunschweig

${ }^{2}$ Rheinische Friedrich-Wilhelms-Universität, Bonn

${ }^{3}$ Heinrich-Heine-Universität, Düsseldorf

${ }^{4}$ Privates Forschungs- und Beratungsinstitut für angewandte Ethik und Tierschutz INSTET gGmbH, Berlin

${ }^{5}$ Fachhochschule Südwestfalen, Soest

${ }^{6}$ Copenhagen Business School, Zeppelin Universität, Friedrichshafen

${ }^{7}$ Technische Universität München

${ }^{8}$ Georg-August-Universität, Göttingen

inken.christoph@thuenen.de

In Deutschland und den übrigen EU-Mitgliedstaaten hat die landwirtschaftliche Nutztierhaltung seit der Jahrtausendwende erheblich an gesellschaftlicher Akzeptanz verloren (European Commission 2005, 2016). Als Reaktion auf den Akzeptanzverlust stellte das Bundesministerium für Ernährung und Landwirtschaft (BMEL) im Juni 2017 seine „Nutztierhaltungsstrategie“ vor (BMEL 2017a) und auch hier wird die wachsende Kritik der Gesellschaft betont. Die folgenden Beiträge, die sämtlich aus dem durch die Innovationsförderung des BMEL geförderten Projekt „SocialLab - Nutztierhaltung im Spiegel der Gesellschaft“ stammen, unterstreichen diesen Punkt und verdeutlichen sowohl die Relevanz der Einbeziehung unterschiedlicher gesellschaftlicher Gruppen wie auch den notwendigen Dialog zwischen diesen Gruppen. Von Seiten der Gesellschaft sind ethische Überlegungen in Bezug auf den Umgang mit Nutztieren von Relevanz, was durch verschiedene Autoren empirisch gezeigt werden konnte (Ohl und van der Staay 2012; Spooner et al. 2014). Dabei handelt es sich keineswegs um eine auf Deutschland beschränkte Debatte (Tonsor et al. 2009; Vanhonacker et al. 2012). Bereits 2006 sahen 77\% der in einer EU-weiten Studie befragten Bürger ${ }^{1}$ die Notwendigkeit, das Wohl landwirtschaftlicher Nutztiere besser zu schützen (European Commission 2007). Dieser Anteil stieg in einer erneuten Umfrage im Jahr 2015 auf 82\% (European Commission 2016). Medienberichte über die Nutztierhaltung zeigen oftmals kritische Bedingungen für die Tiere und lösen bei einer Vielzahl von Bürgern Entsetzen und Ablehnung über die dargestellte Tierhaltung aus (Boehm et al. 2010; Thompson et al. 2011; Spiller et al. 2012). Im Fokus der öffentlichen Kritik stehen vor allem die Schweine- und Geflügelhaltung (European Commission 2005; Vanhonacker et al. 2009; Kayser et al. 2012; Wildraut et al. 2015), während die Milchviehhaltung im Vergleich durch befragte Bürger besser bewertet wird (European Commission 2005; Evans und Miele 2008; Boogaard et al. 2011). In Bezug auf die Schweinehaltung besteht weitgehender Konsens, dass das Platzangebot sowie die Bodenbeschaffenheit von zentraler Bedeutung sind (Kayser et al. 2012; Wildraut et al. 2015; Weible et al. 2016). Darüber hinaus werden die Licht- und

\footnotetext{
$\overline{1}$ Zur besseren Lesbarkeit werden im Folgenden lediglich die männlichen Bezeichnungen gewählt. Selbstverständlich sind Männer und Frauen gleichermaßen gemeint.
} 
Klimaverhältnisse, vorzugsweise mit Außenklimareizen (BMEL 2015b) sowie angebotene Spiel- und Beschäftigungsmöglichkeiten als besonders relevant betrachtet (Ermann et al. 2016). Die Geflügelhaltung wird ebenfalls häufig sehr skeptisch gesehen (Verbeke und Viaene 2000; Vanhonacker und Verbeke 2009; Heng et al. 2013). Neben hohen Besatzdichten, dem Einsatz von Antibiotika und der Größe der Bestände, ist die Tötung von männlichen Eintagsküken ein von der Gesellschaft zunehmend beachtetes Thema (Bruijnis et al. 2015).

Aber auch die Milchviehhaltung ist nicht unumstritten. Im Vordergrund der Kritik steht vor allem das unzureichende Platzangebot, die seltene Weidehaltung, der hohe Einsatz von Kraftfutter zur Optimierung der Milchleistung sowie die vermutete prophylaktische Gabe von Medikamenten (ChristophSchulz et al. 2015) und schließlich das betäubungslose Enthornen der Kälber (Gauly 2015). Dass Bürger die Milchviehhaltung jedoch durchaus ambivalent beurteilen und sowohl Vor- als auch Nachteile sehen, zeigen Boogaard et al. (2011): So wird der heutzutage hohe hygienische Standard in als „modern“ bezeichneten Betrieben durchaus befürwortet, während gleichzeitig der Wunsch nach „traditionellen“ Betrieben besteht. In Bezug auf die Rinderhaltung gibt es bisher weniger kritische Diskussionen. Bioproduktion, verbesserte Haltungsbedingungen und eine grasbasierte Fütterung wirken positiv auf die Kaufpräferenz von Rindfleisch (Risius und Hamm 2017), während über die Intensivmast bisher kaum debattiert wird. Die zunehmende Kritik hat auch ökonomische Auswirkungen auf die Branche. Fleisch und Fleischprodukte stellten zwar 2015 mit einem Anteil von 23,5\% am Gesamtumsatz die wichtigste und Milch und Milchprodukte (ohne Speiseeis) mit knapp 14\% die zweitwichtigste Produktgruppe der deutschen Ernährungsindustrie dar (BVE 2016), allerdings stagniert der Konsum seit vielen Jahren bzw. geht leicht zurück (BMEL 2015a). Die Proteste gegen die vorherrschenden Haltungsbedingungen nehmen gleichzeitig zu (Laine et al. 2017). Der Anteil der Vegetarier in der deutschen Bevölkerung ist von knapp 2\% Mitte der 2000er Jahre auf 4-5\% 10 Jahre später gestiegen (MRI 2008; Cordts et al. 2013; Mensink et al. 2016).

Schlüsselakteure - u.a. das BMEL, die Agrar- und Ernährungswirtschaft, der Lebensmitteleinzelhandel und einige NGOs - versuchen in jüngerer Zeit verstärkt, mit unterschiedlichen Maßnahmen den gesellschaftlichen Anliegen Rechnung zu tragen und in erster Linie das Tierwohl zu verbessern, um so die gesellschaftliche Akzeptanz der Nutztierhaltung zu erhöhen. Beispiele hierfür sind das im Jahr 2013 eingeführte Tierschutzlabel des Deutschen Tierschutzbundes, die in 2015 gestartete Brancheninitiative „Initiative Tierwohl“, das ebenfalls in 2015 veröffentlichte Gutachten des Wissenschaftlichen Beirats für Agrarpolitik beim Bundesministerium für Ernährung und Landwirtschaft zum Tierwohl (BMEL 2015b) oder das geplante staatliche Tierwohl-Label, dessen Kriterien im April 2017 vorgestellt wurden (BMEL 2017b). Zudem wächst die Erkenntnis, dass eine wissenschaftliche Fundierung der politischen Maßnahmen dazu beitragen könnte, mögliche Fehlentwicklungen frühzeitig zu identifizieren und evidenzbasiert zu gestalten (Oehler et al. 2013).

Vor diesem Hintergrund schlossen sich im Herbst 2012 die Autoren dieses Beitrags und Wissenschaftler aus ihren Teams zu einem Konsortium zusammen, um das Thema "Bewertung und Akzeptanz der Nutztierhaltung in Deutschland" im Rahmen der Projektgruppe „SocialLab ${ }^{2}$ strukturiert und umfassend mit unterschiedlichen, aufeinander abgestimmten Methoden zu untersuchen (ThünenInstitut 2015). Sämtliche Partner des Konsortiums arbeiten seit Jahren zu Themen der Agrarökonomik, Verbraucherforschung, Verhaltensökonomik und/ oder Tierethik und decken gemeinsam ein breites Theorien- und Methodenspektrum ab. Für jede Forschungsfrage innerhalb des Projektes wird dabei auf die Kombination mehrerer, adäquater Methoden geachtet. Der Methodenbaukasten, der im SocialLab verwendet wird, besteht aus qualitativen und quantitativen Methoden, aber auch aus experimentellen Verfahren. Zu den qualitativen Methoden zählen u. a. Gruppendiskussionen, Experten- und Tiefeninterviews. Quantitative Methoden sind z. B. durch standardisierte Befragungen und Panelanalysen vertreten. $\mathrm{Zu}$ den experimentellen Methoden gehören u. a. bildgebende Verfahren der Consumer Neuroscience und die Blickregistrierung. Mit dieser Vorgehensweise ist es möglich, im Rahmen des SocialLab Projektes die Komplexität der Nutztierhaltung, so wie sie heute in der Gesellschaft gesehen wird, adäquat zu würdigen und abzubilden. Das Projekt stellt in seiner Breite auch weltweit eine Innovation dar.

$\mathrm{Zu}$ betonen ist, dass das Ziel des SocialLab Teams ist, evidenzbasiert Parameter der Akzeptanz für eine gesellschaftlich akzeptierte und konsensfähige Nutztierhaltung $\mathrm{zu}$ erforschen und in konkrete Politikempfehlungen zu überführen. In diesem Prozess werden sowohl die Perspektive der

\footnotetext{
${ }^{2}$ www.sociallab-nutztiere.de Zugriff am 2.11.2017.
} 
Landwirtschaft und des Handels, als auch die der Verbraucher berücksichtigt, um in komplexen und langfristig wirkenden Zusammenhängen konkrete Politikgestaltungsmöglichkeiten zu erarbeiten. Die Erreichung dieses Ziels setzt eine längerfristig angelegte Forschung der Partner des Konsortiums im Verbund voraus. Damit folgt das Projekt „SocialLab“ in weiten Teilen der Strategie der Deutschen Agrarforschungsallianz (DAFA 2012).

Im Rahmen dieses „Reports“ werden der (Fach-) Öffentlichkeit erste Ergebnisse des Projektes präsentiert und Implikationen für die weitere Forschung abgeleitet. Im Folgenden werden die verschiedenen Schwerpunkte der einzelnen Beiträge kurz skizziert. Im Mittelpunkt der Beiträge von Simons et al., Kühl et al., Rovers et al. und Wildraut und Mergenthaler stehen Fragen, wie die Nutztierhaltung durch die Gesellschaft und gesellschaftliche Gruppen akzeptiert bzw. wahrgenommen wird und welche Anforderungen an die Tierhaltungsverfahren sich aus der gesellschaftlichen Diskussion ableiten lassen. Die Erkenntnisse dieser Studien bilden eine wichtige Grundlage für die folgenden Analysen, die die hier gewonnenen Erkenntnisse in ihre Arbeiten mit einbeziehen. Die Grundlagenstudie von Simons et al. zeigt, dass die Wahrnehmung der Tierhaltung durch eine Bildkonfiguration charakterisiert ist, in der das Bild einer „heilen Welt“ (,Museumslandwirtschaft“) dem einer „Schreckenswelt“ (,Massentierhaltung“) gegenübersteht. Während die „Museumslandwirtschaft" sich durch einen als fair empfundenen Deal zwischen Mensch und Tier auszeichnet, steht die „Massentierhaltung“ für einen unwürdigen Umgang mit den Tieren. Die Konfiguration ist stark durch mediale Berichte und sowohl von Sehnsüchten als auch von Schreckensphantasien und Deutungsmustern beeinflusst.

In der Arbeit von Kühl et al. werden für Milchkühe, Mastschweine und Masthähnchen vier verschiedene, für die Praxis wichtige Haltungssysteme (Stallhaltung, Außenklimastall, Stallhaltung mit Auslauf und Stallhaltung mit Weidegang im Sommer) in systematischer Form auf ihre Bewertung und Akzeptanz durch die Bürger verglichen. Die Ergebnisse verdeutlichen, dass die Gesellschaft die reine Stallhaltung für Nutztiere sehr kritisch bewertet. Für alle Tierarten zeigt sich eine klare Präferenz für Haltungssysteme, die den Tieren zumindest Außenklima ermöglichen. Dabei wird die Weidehaltung am besten bewertet. Die Ergebnisse zeigen Forschungsbedarf hinsichtlich innovativer Haltungssysteme auf, die die Anforderungen der Bürger aufgreifen.
Der Beitrag von Rovers et al. bietet Einblicke in die Wahrnehmung der landwirtschaftlichen Nutztierhaltung von Rind, Schwein und Huhn durch Landwirte und Bürger. Dabei zeigen sich tierartenspezifische, aber auch tierartenübergreifende Unterschiede, z.B. beim Einsatz von Technik im Stall. Während Landwirte die Arbeitserleichterung und positive Effekte für das Tierwohl durch eine bessere Tierversorgung und -überwachung betonen, gehen die Bürger von einem verminderten Mensch-TierKontakt aus.

Der Beitrag von Wildraut und Mergenthaler baut auf dem Beitrag von Rovers et al. insofern auf, dass die in den Gruppendiskussionen identifizierten Konflikte zur Nutztierhaltung gemeinsam mit Landwirten und Verbrauchern diskutiert wurden, um konkret zu untersuchen, wie beide Gruppen in der direkten Konfrontation zu diesen Konflikten stehen. Die Ergebnisse zeigen u.a., dass insbesondere Verbraucher, aber in Grenzen auch Landwirte bereit sind, ihre Einschätzungen zu ändern, wenn sie die Sichtweise der anderen Gruppe hören.

Der zweite Abschnitt dieses Beitrags umfasst die Arbeiten von Gier, Krampe et al., Gier et al. sowie von Groß und Roosen. Übergeordnet geht es um die Systematisierung und Untersuchung vorhandener Informationen, deren Wirkung und der Ableitung von Hinweisen für die zukünftige Gestaltung von Verbraucherinformationen. Die Arbeiten bauen auf den Erkenntnissen des ersten Arbeitspakets auf und erweitern diese u.a. um die Wirkung sachlicher und emotionaler Informationen auf Verbraucher. Über diese Erkenntnisse hinaus können beispielsweise die Ergebnisse zur Wahrnehmung wertvolle Ergänzungen bieten. Dies trägt fundamental dazu bei, das komplexe Zusammenspiel der unterschiedlichen Einflussfaktoren auf die Wahrnehmung und Akzeptanz der landwirtschaftlichen Nutztierhaltung zu durchdringen und Wege aufzuzeigen, wie diese beeinflusst werden können.

Gier, Krampe et al. befassen sich im ersten Beitrag mit den Informationen, die Verbraucher tagtäglich beim Einkauf tierischer Produkte in Bild- und Textform wahrnehmen. Zudem vergleichen sie die neuralen Wirkungen von Kommunikationsmaßnahmen in unterschiedlichen Produktionsformen (biologische, konventionelle Haltung). Die Autoren finden z.B. heraus, dass sich für biologisch-orientierte Kommunikationsmaßnahmen ein signifikant höherer durchschnittlicher Fleischwaren-Wochenumsatz pro Kunde ergibt.

Der zweite Artikel von Gier et al. befasst sich mit dem Labelling und den Möglichkeiten, aber auch 
Grenzen dieser besonderen Art der Verbraucherkommunikation. Verbraucher beschreiben in dieser Studie Labels als häufig unverständlich und die Labelvielfalt als eher lästig. Darüber hinaus wird als innovativer Ansatz ein „Verbraucherinformationssystem“ skizziert, mit dem informationslogistische Ineffizienzen reduziert werden könnten.

Der Beitrag von Groß und Roosen untersucht den Einfluss von Nachrichtentexten auf das soziale Vertrauen von Verbrauchern in Landwirte im Kontext der Nutztierhaltung. Personen, die zunächst ein geringes (hohes) Vertrauen in Landwirte haben, reagieren auf den Erhalt einer Nachricht positiv (negativ). Negative Nachrichten haben einen stärkeren Effekt als positive.

Der dritte große Abschnitt dieses Beitrages untersucht die Sichtweise spezifischer Akteure entlang der Wertschöpfungskette sowie die ökonomischen Auswirkungen von obligatorischen und/oder freiwilligen Tierschutzstandards. Die in den ersten zwei Arbeitspaketen gewonnenen Erkenntnisse werden weiter konkretisiert, indem Motive, aber auch Hemmnisse dafür untersucht werden, Produkte mit strengeren Tierschutzstandards zu produzieren, in das Sortiment aufzunehmen bzw. zu kaufen.

Wildraut und Mergenthaler untersuchen die Bereitschaft von Landwirten, derzeitige Tierhaltungsverfahren hinsichtlich mehr Tierwohl weiterzuentwickeln. Aus Sicht der Landwirte könnten die Haltungsverfahren in Deutschland weiter verbessert werden, Ideen werden allerdings aufgrund politischer und wirtschaftlicher Einschränkungen sowie persönlicher und beruflicher Vorbehalte zurückhaltend formuliert.

Der zweite Beitrag von Krampe et al. betrachtet das Meinungsbild des Lebensmitteleinzelhandels und geht explizit auf Fragen zur Listungsentscheidung, zur staatlichen Regulierung bei der Durchsetzung und Integration höherer Tierschutzstandards in die Wertschöpfungskette sowie auf den Einfluss von Labels auf die Verbraucher ein.

Roosen et al. analysieren die Präferenzen der Verbraucher bezüglich angemessener Tierwohlregulierungen für Masthühner und die Verantwortlichkeiten hierfür ebenso wie die Reaktion auf steigende Fleischpreise. Die Ergebnisse betonen die Problematik der Tötung von Eintagsküken. So werden Zweinutzungshühner im Vergleich zur Geschlechtsbestimmung im Ei präferiert. Landwirte werden als Hauptverantwortliche für das Tierwohl identifiziert.
Das vierte und abschließende Arbeitspaket ermittelt die Ansprüche der Verbraucher an die Tierhaltungsverfahren in Hinblick auf entstehende Zielkonflikte und prüft die Möglichkeiten der Umsetzbarkeit. Dabei baut es sehr stark auf den ersten drei Arbeitspaketen auf. Außerdem wird die gesellschaftliche Bewertung innovativer Tierhaltungsverfahren betrachtet.

Sonntag et al. untersuchen, wie Bürger reagieren, wenn sie mit Zielkonflikten konfrontiert werden, die zwischen verschiedenen Nachhaltigkeitszielen bestehen, wie etwa zwischen dem Tierwohl auf der einen und der Tiergesundheit oder dem Umweltschutz auf der anderen Seite. Die Autoren zeigen mit ihrer Untersuchung, dass das Wohl der Tiere dominiert. So ist ein hoher Tierwohlstatus von größerer Bedeutung als bspw. ein geringer Verbraucherpreis oder die Produktqualität. In gleichem Maße entschieden sich Bürger für das Wohl der Tiere auch zu Lasten von anderen Nachhaltigkeitszielen, wie z.B. dem Umweltschutz.

Brümmer et al. befassen sich mit dem Zweinutzungshuhn und ermitteln u.a., dass das Kükentöten zwar abgelehnt wird, die befragten Verbraucher aber nicht bereit sind, eigene Konsumgewohnheiten deutlich $\mathrm{zu}$ ändern. Die Ergebnisse dieses Beitrages wurden durch die enge Zusammenarbeit mit dem Projekt „IntegHof Geflügelhaltung neu strukturiert" gewonnen, das sich aus naturwissenschaftlicher sicht mit dem Zweinutzungshuhn befasst und an der Tierärztlichen Hochschule in Hannover koordiniert wird.

\section{Danksagung}

"SocialLab - Nutztierhaltung im Spiegel der Gesellschaft" wird aus Mitteln des Bundesministeriums für Ernährung und Landwirtschaft (BMEL) aufgrund eines Beschlusses des deutschen Bundestages gefördert. Die Projektträgerschaft erfolgt über die Bundesanstalt für Landwirtschaft und Ernährung (BLE) im Rahmen des Programms zur Innovationsförderung (FKZ: 2817202813). SocialLab Deutschland ist ein Zusammenschluss folgender Partner: Heinrich-Heine-Universität Düsseldorf, Thünen-Institut für Marktanalyse (Gesamtkoordination), Georg-August-Universität Göttingen, Rheinische Friedrich-Wilhelms-Universität Bonn, Fachhochschule Südwestfalen Soest, Technische Universität München, Privates Forschungs- und Beratungsinstitut für angewandte Ethik und Tierschutz INSTET GmbH. Darüber hinaus danken wir den Personen und Institutionen, die uns im Rahmen der 
jeweiligen Teilprojekte unterstützt haben sowie insbesondere den Mitgliedern des SocialLab-Beirats (vgl. www.sociallab-nutztiere.de).

\section{Literatur}

BMEL (2015a) Versorgung mit Fleisch nach Fleischarten. http://www.ble.de/DE/BZL/Daten-Berichte /Fleisch/fleisch.html. Abgerufen 19.10.2017

BMEL (2015b) Wege zu einer gesellschaftlich akzeptierten Nutztierhaltung. http://www.bmel.de/Sha redDocs/Downloads/Ministerium/Beiraete/Agrarp olitik/GutachtenNutztierhaltung.pdf?_blob=pub licationFile. Abgerufen 19.10.2017

BMEL (2017a) Nutztierhaltungsstrategie. Zukunftsfähige Tierhaltung in Deutschland. http://www. bmel.de/SharedDocs/Downloads/Tier/Nutztierhalt ungsstrategie.pdf?_blob=publicationFile. Abgerufen 19.10.2017

BMEL (2017b) Fragen und Antworten zum staatlichen Tierwohllabel. http://www.bmel.de/DE/Tier/Tierw ohl/_texte/Tierwohllabel-Fragen-und-Antworten. html. Abgerufen 19.10.2017

Boehm J, Kayser M, Spiller A (2010) Two sides of the same coin? Analysis of the web-based social media with regard to the image of the agri-food sector in Gemany. Int J Food Syst Dyn 3:264-278

Boogaard BK, Bock BB, Oosting SJ, Wiskerke JSC, van der Zijpp AJ (2011) Social acceptance of dairy farming: the ambivalence between the two faces of modernity. J Agric Environ Ethics 24(3): 259-282

Bruijnis MRN, Blok V, Stassen EN, Gremmen HGJ (2015) Moral "Lock-In" in responsible innovation: the ethical and social aspects of killing day-old chicks and its alternatives. J Agric Environ Ethics 28(5):939-960

BVE (2016) Jahresbericht 2015_2016. http://www.bveonline.de/presse/infothek/publikationen-jahresberi cht. Abgerufen 19.10.2017

Christoph-Schulz I, Salamon P, Weible D (2015) What is the benefit of organically reared dairy cattle? Societal perception towards conventinal and organic dairy farming. Int J Food Syst Dyn 6(3):139-146

Cordts A, Spiller A, Nitzko S, Grethe H, Duman N (2013) Fleischkonsum in Deutschland. Von unbekümmerten Fleischessern, Flexitariern und (Lebensabschnitts-) Vegetariern. FleischWirtschaft 7:59-63

DAFA (2012) Fachforum Nutztiere. http://www.dafa. de/fileadmin/dam_uploads/images/Fachforen/Bro
sch-DAFA-FFNutztiereWeb.pdf. Abgerufen 19.10 . 2017

Ermann M, Graskemper V, Spiller A (2016) Die Wirkung von geführten Stallbesichtigungen auf Bürger - eine Fallstudie auf nordwestdeutschen Schweinemastbetrieben. In: Schriften der Gesellschaft für Wirtschafts- und Sozialwissenschaften des Landbaues e.V., Band Nr. 52: Agrar- und Ernährungswirtschaft: Regional vernetzt und global erfolgreich, 45-56

Evans A, Miele M (2008) Consumers' views about farm animal welfare. Part II: European comparative report based on focus group research. Welfare quality Reports No. 5, ISSN 1749-5164

European Commission (2005) Attitudes of consumers towards the welfare of farmed animals, Special Eurobarometer 229. http://ec.europa.eu/commfro ntoffice/publicopinion/archives/ebs/ebs_229_en.pdf. Abgerufen 19.10.2017

European Commission (2007) Attitudes of EU citizens towards Animal Welfare, Special Eurobarometer 270. http://ec.europa.eu/commfrontoffice/publicop inion/archives/ebs/ebs_270_en.pdf. Abgerufen 19.10.2017

European Commission (2016) Attitudes of Europeans towards Animal Welfare, Special Eurobarometer 442. http://www.izs.it/IZS/Engine/RAServeFile.php/ f/pdf_vari_grafica_/Attitudes_of_Europeans_towar ds_Animal_Welfare.pdf. Abgerufen 19.10.2017

Gauly M (2015) Was können wir in der Milchviehhaltung besser machen? Die bayerische Milchwirtschaft im freien Wettbewerb 5(2015):8-14

Heng Y, Peterson H, Li X (2013) Consumer attitudes towards farm-animal welfare: the case of laying hens. J Agric Resour Econ 38(3):418-434

Kayser M, Schlieker K, Spiller A (2012) Die Wahrnehmung des Begriffs „Massentierhaltung“ aus Sicht der Gesellschaft. Ber über Landwirtsch 90(3):417-428

Laine M, Laine M, Vinnari E, Vinnari E (2017) The transformative potential of counter accounts: a case study of animal rights activism. AAAJ, 30(7):1481-1510

Mensink GBM, Barbosa CL, Brettschneider AK (2016) Verbreitung der vegetarischen Ernährungsweise in Deutschland. J Health Monitor 1(2):2-15

MRI (Max Rubner-Institut, Bundesforschungsinstitut für Ernährung und Lebensmittel) (Hrsg., 2008) Nationale Verzehrsstudie II. Ergebnisbericht

Oehler A, Kenning P (2013) Evidenzbasierung ermöglichen! Auf dem Weg zu einer realitätsnahen und empirisch fundierten Verbraucherpolitik. 
Stellungnahme des wissenschaftlichen Beirats Verbraucher- und Ernährungspolitik beim BMELV, September 2013 (mit Anmerkungen von: Kornelia Hagen, Christa Liedtke, Ulf Schrader)

OHL F, van der Staay FJ (2012) Animal welfare: At the interface between science and society. Vet J 192, 13-19

Risius A, Hamm U (2017) The effect of information on beef husbandry systems on consumers' preferences and willingness to pay. Meat Sci 124:9-14

Spiller A, Kayser M, Böhm J (2012) Unternehmerische Landwirtschaft zwischen Marktanforderungen und gesellschaftlichen Erwartungen in Deutschland aus Sicht der Forschung. Schriften der Gesellschaft für Wirtschafts- u. Sozialwissenschaften des Landbaues e.V. 47, 11-22

Spooner JM, Schuppli CA, Fraser D (2014) Attitudes of Canadian citizens toward farm animal welfare: a qualitative study. Livest Sci 163:150-158

Thünen-Institut (2015) Pressemitteilung, Nutztierhaltung im Spiegel der Gesellschaft. https://www. thuenen.de/de/infothek/presse/pressearchiv/presse mitteilungen-2015/nutztierhaltung-im-spiegel-dergesellschaft/. Abgerufen 19.10.2017

Tonsor GT, Olynk N, Wolf C (2009) Consumer preferences for animal welfare attribute: The case of gestation crates. J Agric Appl Econ 41(2):713-730

Thompson, PB, Appleby M, Busch L, Kalof, L, Miele, M, Norwood BF, Pajor E (2011) Values and public acceptability dimensions of sustainable egg production. Poult Sci 90:2097-2109
Vanhonacker F, Verbeke W, van Poucke E, Pieniak Z, Nijs G, Tuyttens F (2012) The concept of farm animal welfare: citizen perceptions and stakeholder opinion in flanders, Belgium. J Agric Environ Ethics 25(1):79-101

Vanhonacker F, Verbeke W, van Poucke E, Buijs S, Tuyttens F (2009) Societal concern related to stocking density, pen size and group size in farm animal production. Livest Sci 123:16-22

Vanhonacker F, Verbeke W (2009) Buying higher welfare poultry products? Profiling Flemish consumers who do and do not. Poult Sci 88(12):2702-2711

Verbeke W, Viaene J (2000) Ethical challenges for livestock production: Meeting consumer concerns about meat safety and animal welfare. J Agric Environ Ethics 12(2):141-151

Weible D, Christoph-Schulz I, Salamon P, Zander K (2016) Citizens' perception of modern pig production in Germany: a mixed-method research approach. Brit Food J 118(8):2014-2032

Wildraut C, Plesch G, Härlen I, Simons J, Hartmann M, Ziron M, Mergenthaler M (2015) Multimethodische Bewertung von Schweinehaltungsverfahren durch Verbraucher anhand von Videos aus realen Schweineställen. Landwirtschaftliche Fakultät der Universität Bonn, Schriftenreihe des Lehr- und Forschungsschwerpunktes USL, Nr. 179 


\section{Akzeptanz der Nutztierhaltung in Deutschland - Ergebnisse der psychologischen und ethischen Untersuchung von Bestimmungsfaktoren}

Johannes Simons ${ }^{1}$, Jörg Luy ${ }^{2}$, Carl Vierboom ${ }^{3}$, Ingo Härlen ${ }^{1}$, Jeanette Klink-Lehmann ${ }^{1}$ und Monika Hartmann ${ }^{1}$

${ }^{1}$ Rheinische Friedrich-Wilhelms-Universität Bonn, Institut für Lebensmittel- und Ressourcenökonomik, Professur für Marktforschung der Agrar- $u$. Ernährungswirtschaft

${ }^{2}$ Privates Forschungs- und Beratungsinstitut für angewandte Ethik und Tierschutz INSTET gGmbH, Berlin

${ }^{3}$ Wirtschaftspsychologen Vierboom \& Partner, Hennef (Sieg)

johannes.simons@ilr.uni-bonn.de

\section{Zielsetzung und Einordnung}

Die Zielsetzung der nachfolgend dargestellten Grundlagenstudie bestand in der Analyse der gesellschaftlichen Akzeptanzbildung dessen, was als derzeitige Tierhaltung in Deutschland wahrgenommen wird. Hierzu war es notwendig, die Wahrnehmung als Grundlage der Akzeptanzbildung in die Untersuchung einzubeziehen. Die Ergebnisse basieren auf Gruppendiskussionen und Tiefeninterviews, in denen die Sicht der jeweiligen Teilnehmer auf das Thema Tierhaltung im Vordergrund steht. Sie sollen zu einem besseren Verständnis der Wahrnehmung der Tierhaltung und der Akzeptanzbildung beitragen und als Interpretationsrahmen für weitere Untersuchungen dienen.

\section{Vorgehensweise}

Die Analyse erfolgte auf Basis der Morphologischen Psychologie, die auf die Erklärung der Dynamik psychischer Prozesse ausgerichtet ist. Sie konzentriert sich auf die zugrundeliegenden, unterschiedlichen, zum Teil gegensätzlichen und zueinander in einem Spannungsverhältnis stehenden Motive (Ziems 2004). Die Morphologische Psychologie geht davon aus, dass unbewusste und vorbewusste Prozesse Verhalten und Wahrnehmung steuern. In Einklang mit der psychoanalytischen Theorie soll ein umfassender Ansatz die jeweiligen psychischen Phänomene erklären (Lönneker 2011). In der Analyse konzentriert sich die Morphologische Psychologie dabei nicht auf personenorientierte Konzepte, sondern darauf, welche Wirkungen von einem spezifischen Produkt oder einer Idee ausgehen können.
Als Erhebungsmethode dienen Tiefeninterviews und Gruppendiskussionen, in denen die Teilnehmer ihre Ansichten und Erfahrungen aus dem Alltagsleben beschreiben. Aus diesen Beschreibungen lassen sich die unbewussten oder vorbewussten Zusammenhänge erkennen (Melchers und Ziems 2001).

Die Qualität der Ergebnisse hängt ab von der Fähigkeit des Forschers, die Schilderungen in den Interviews empathisch zu verstehen (Fitzek 2010). Der Ansatz nutzt explizit den Forscher und sein Empathievermögen als grundlegende Voraussetzung für das Verständnis der relevanten Zusammenhänge. Eine spezifische Schulung der Forscher ist Voraussetzung, um die verdeckten Motive und Emotionen aufdecken zu können (Reik 1983). Eine solche, der Zielsetzung dieser Untersuchung angemessene Vorgehensweise ist notwendigerweise subjektiv. Um mögliche Verzerrungen der Ergebnisse durch das emotionale Erleben der Interviewer methodisch zu kontrollieren, werden die Untersuchungen in einem Team von Forschern durchgeführt, diskutiert und ausgewertet.

Interviews und Gruppendiskussionen dauern in der Regel eineinhalb bis zwei Stunden. Dieser Zeitrahmen kann nicht genügen, um die persönliche Motivstruktur des Teilnehmers genau zu untersuchen - was auch nicht das Ziel der Untersuchung war. Er ist aber ausreichend, um unterschiedliche Facetten der Wahrnehmung der Tierhaltung zu beleuchten, z.B. unterschiedliche Bilder von der Tierhaltung und deren Akzeptanz.

\section{Stichprobe}

Für eine umfassende Analyse muss die Rekrutierung der Teilnehmer so vorgenommen werden, dass alle hypothetisch relevanten Aspekte von wenigstens einem, besser aber von mehreren Teilnehmern angesprochen werden. Als hypothetisch relevant für die Akzeptanz der Nutztierhaltung wurden regionale Einflüsse und die Grundhaltung zum Fleischverzehr eingeschätzt. Um diese Unterschiede abzudecken, wurden die Interviews in verschiedenen Regionen bzw. Städten Deutschlands durchgeführt: in den Großstädten Berlin und Bochum, in Oldenburg, einer Stadt im Zentrum der deutschen Schweine-, Geflügelund Eierproduktion, in Kempten, einer Stadt im Voralpenland in einer Ferienregion mit intensiver Milchproduktion, in Göttingen im Zentrum Deutschlands sowie Erfurt im Osten Deutschlands als zwei Regionen mit geringer Tierdichte. In Köln, einer weiteren Großstadt, wurde lediglich eine 
Gruppendiskussion veranstaltet. Zwei der Gruppendiskussionen (Berlin und Köln) sowie vier Einzelinterviews an jeweiligen Orten fanden mit Vegetariern oder Veganern statt. Diskussionen mit Vegetariern und Veganern sowie Fleischessern in einer Gruppe wurden nicht organisiert aus der Erwartung heraus, dass bei einer solchen Konstellation die Auseinandersetzung zwischen den Gruppen und nicht mehr die Wahrnehmung der Tierhaltung im Vordergrund stehen würde.

Der Gesamtumfang der Stichprobe beträgt 116 Personen $^{3}$ mit 67 Teilnehmerinnen und 49 Teilnehmern. 42 Personen waren zwischen 20 und 30 Jahren, 37 zwischen 31 und 50 und 30 zwischen 51 und 73. Sieben Personen machten keine Altersangaben.

Die Rekrutierung der Teilnehmer erfolgte durch ein kommerzielles Marktforschungsinstitut. Die Erhebung fand im Zeitraum zwischen September und Dezember 2015 statt. Vorab wurden die Teilnehmer lediglich darüber informiert, dass Landwirtschaft und Ernährung die Themen der Interviews bzw. der Diskussionen sein würden.

\section{Ergebnisse}

Die Beobachtungen in den Interviews und Gruppendiskussionen zeigen vor allem bei Fleischessern einen Unwillen, sich intensiv mit der Tierhaltung auseinanderzusetzen. Die Beschäftigung erscheint oft anstrengend. Unwillen und Anstrengung deuten darauf hin, dass diese Auseinandersetzung im Alltag gemieden wird. Bei Vegetariern und Veganern bestehen demgegenüber tendenziell konkretisierte Vorstellungen über negative Seiten der Tierhaltung, die Belebung von abstoßenden Bildern aus der Tierhaltung fällt deutlich leichter und die Auskunftsbereitschaft ist größer als bei den Fleischessern.

Die Wahrnehmung der Tierhaltung generiert sich aus Bildern und Informationen, die aus Massenmedien, Internet, sozialen Medien, persönlicher Kommunikation und eigenen Erfahrungen stammen und durch Systematisierungen und Schlussfolgerungen in einen Sinnzusammenhang gebracht werden. Persönliche Erfahrungen mit der heutigen Tierhaltung sind dabei eher die Ausnahme.

\footnotetext{
${ }^{3} 57$ Einzelinterviews, ein Interview mit 2 Personen und 7 Gruppendiskussionen (2 Gruppen mit je 9 Teilnehmern, 2 Gruppen mit je 8 Teilnehmern und weitere 3 Gruppen mit je 6 , 7 oder 10 Teilnehmern).
}

\subsection{Grundlegende Bildfiguration}

Die Analyse der Wahrnehmung von Tierhaltung ergibt eine Figuration, die aus einem schönen und geliebten Bild von Tieren und Tierhaltung auf der einen Seite sowie aus Schock- und Schreckensbildern auf der anderen Seite besteht. Im Folgenden werden diese beiden Seiten mit den Begriffen Museumslandwirtschaft und Massentierhaltung ${ }^{4}$ bezeichnet.

Museumslandwirtschaft: Die Bilder der Museumslandwirtschaft sind auf eine heile Welt der Tierhaltung und der mit der Tierhaltung befassten Personen ausgelegt. Charakteristisch ist ein wertschätzender Umgang mit den Tieren, der sich in einer als tiergerecht wahrgenommenen Haltung äußert. Halter und Tiere werden häufig als gutmütig und geduldig beschrieben, die im Sinne eines ,fairen Deals" zusammenleben und aufeinander angewiesen sind. Den Hintergrund für diese Bilder bieten Vorstellungen von Höfen aus der Vergangenheit oder von solchen Betrieben, die die Entwicklung zur modernen Landwirtschaft nicht mitgemacht haben. Traditionen, Beständigkeit, Kindheitserinnerungen oder Vorstellungen von einer glücklichen Kindheit sind wichtige Bestandteile.

Gespeist werden die Bilder der Museumslandwirtschaft durch Medienberichte, Heimatfilme und Werbung, durch eigene Sehnsüchte und Erfahrungen, durch Erlebnisse wie dem Urlaub und das Einkaufen auf dem Bauernhof oder durch die Teilnahme an traditionsreichen landwirtschaftlichen Festen. Die Museumslandwirtschaft steht aufgrund des als fair empfundenen Deals zwischen Mensch und Tier für eine akzeptierte Form der Tierhaltung. Fragen nach der umfassenden Realisierbarkeit dieser Art von Landwirtschaft oder der Vollständigkeit der Vorstellungen z.B. im Hinblick auf Arbeitsverhältnisse und Tierwohl spielen bei der Faszination, wenn überhaupt, nur eine untergeordnete Rolle. Vielmehr sind die Bilder Ausdruck des Wunsches, komplexe Zusammenhänge auf eine einfache und überschaubare Ordnung $\mathrm{zu}$ bringen. Sie sprechen die Sehnsucht nach Beständigkeit und geringer Komplexität der eigenen Lebensverhältnisse an und können damit als Gegenbilder zu einem hektisch und kompliziert erlebten Alltag dienen. Für die

\footnotetext{
${ }^{4}$ Obwohl der Begriff „Massentierhaltung“ ursprünglich von der Bundesregierung wertneutral als „Haltung großer Nutztierbestände auf begrenztem Raum in neuzeitlichen Haltungssystemen“ definiert wurde (Bundesregierung 1971, S. 9), hat sich die Rede von der „Massentierhaltung“ zum Inbegriff moralischen Fehlverhaltens in Bezug auf Tiere entwickelt.
} 
gewöhnlichen Anforderungen des Alltags ist die Museumslandwirtschaft allerdings zu begrenzt.

Massentierhaltung: Der Museumslandwirtschaft steht die Massentierhaltung gegenüber. Charakteristisch sind dunkle, enge Ställe, in denen Tiere vor sich hinvegetieren. Der Umgang mit den Tieren wird als nicht wertschätzend und als tierquälerisch wahrgenommenen. Massentierhaltung steht der Bedeutung nach vor allem für Maßlosigkeit, für die Verletzung moralischer Grundsätze und fehlende Individualität. Mit einer Fixierung auf bestimmte Größenordnungen wie der Anzahl der Tiere pro Betrieb lässt sie sich nur unzureichend charakterisieren. In diesem System, das auch die verarbeitende Industrie und z.T. den Handel mit einbezieht, wird unterstellt, dass die Tiere als seelenlose Ware behandelt werden und die Halter entweder den Tieren gegenüber gefühllos sind oder selber Opfer eines ausbeuterischen Systems wurden, in dem sie sich z.B. getrieben von der Fleischwirtschaft - gezwungenermaßen schuldig machen. Das Schreddern von Küken, Kastration, das Kupieren von Schwänzen oder das Schlachten nicht ausreichend betäubter Tiere sind Beispiele, die in diesem Zusammenhang erwähnt werden. Der Einsatz von Antibiotika wird oft als notwendige Maßnahme zur Aufrechterhaltung eines als krank erlebten Systems eingeschätzt. Bei der Beschreibung der Massentierhaltung verwischt die Grenze zwischen dem Umgang mit Tieren und dem Umgang mit Menschen. Stallanlagen werden auch als „Tier-KZ“ bezeichnet und die Behandlung der Arbeiter in den Schlachthäusern als ebenso unwürdig beschrieben wie die der Tiere. Insbesondere bei der Diskussion um den Einsatz von Antibiotika wird befürchtet, dass das als maßlos eingeschätzte System katastrophale Folgen für die menschliche Gesundheit hat. Fleisch, das auf der einen Seite „ein Stück Lebenskraft" darstellt, wird damit zum trojanischen Pferd, das die Möglichkeiten zur Krankheitsbekämpfung aushöhlt. Die Existenz eines Sektors, der sich wenig um moralische Grundsätze oder gesetzliche Regelungen zu kümmern scheint, kann darüber hinaus auch als Zeichen für die allgemeine gesellschaftliche Entwicklung und als Bedrohung für den eigenen Alltag erlebt werden. Die Massentierhaltung ist die nicht akzeptierte Form der Tierhaltung. Sie wird in nahezu allen Interviews angesprochen und bietet aufgrund fehlender Erfahrung mit der Tierhaltung Raum für ausufernde und auch faszinierende Schreckensphantasien. Sie steht mit ihrer beängstigenden Maßlosigkeit der als angenehm empfundenen Begrenzung der Museumslandwirtschaft gegenüber.

\subsection{Einordnung der Massentierhaltung in das Gesamtbild von der Tierhaltung}

Die Existenz der nicht akzeptierten Massentierhaltung wird in der Regel nicht angezweifelt, Unterschiede ergeben sich allerdings bei der Einordnung der Massentierhaltung in den Gesamtkomplex sowohl der Tierhaltung als auch des eigenen Alltags. Hierzu konnten in der Analyse vor allem zwei Ansätze identifiziert werden:

- Massentierhaltung als Skandal in einem ansonsten funktionierenden Versorgungssystem: Hierbei besteht die Vorstellung oder Hoffnung, dass größere Organisationen und Kontrollbehörden das Funktionieren des Systems überwachen, so dass Abweichungen und Störungen weitgehend durch Aufdeckung und durch die Bestrafung der Verursacher vermieden werden.

- Massentierhaltung als Normalität in einem moralisch verwerflichen System: Hierbei sind den Schreckensphantasien über den Umgang mit den Tieren und den Charakter der Menschen kaum Grenzen gesetzt. Entsprechende Äußerungen beziehen sich in der Regel auf mediale Darstellungen der Tierhaltung, die durch eigene Vermutungen, Spekulationen und Verdächtigungen angereichert und auf diese Weise weiterentwickelt werden.

\subsection{Vorstellungen zur Weiterentwicklung der Tierhaltung}

Für die Beurteilung unterschiedlicher Tierhaltungsverfahren und deren Weiterentwicklung bestehen vermeintlich klare Vorstellungen hinsichtlich der Beurteilungskategorien. Vor allem Bewegungsfreiheit, Licht, frische Luft und Beschäftigungsmöglichkeiten werden immer wieder genannt.

\subsection{Persönliche Auseinandersetzung mit der Massentierhaltung}

Die Auseinandersetzung mit den Bildern und Vorstellungen von Massentierhaltung können zu heftigen emotionalen Reaktionen und in der Folge $\mathrm{zu}$ entsprechenden Abwehrreaktionen führen. Es besteht eine starke Tendenz, nichts oder wenig wissen zu wollen, um sich emotionalen Störungen nicht aussetzen zu müssen. In der Untersuchung wurden vor allem folgende Gründe deutlich: 
- Der Genuss von Fleisch und Fleischprodukten wird durch Berichte über Massentierhaltung und die dadurch ausgelöste Konfrontation mit unappetitlichen Bildern eingeschränkt.

- Die Auseinandersetzung mit der Fleischerzeugung verdeutlicht den Verwendern von Tierprodukten eine eigene Verstrickung in ein System, das als maßlos und unmoralisch beurteilt wird.

- Die Schreckensbilder der Massentierhaltung und die in diesem Zusammenhang wahrgenommene Brutalität gegenüber lebenden Kreaturen lösen Ängste um die eigene Verwundbarkeit aus.

Während die ersten beiden Punkte vor allem für Fleischesser gelten, trifft der dritte Punkt auch auf Teilnehmer zu, die sich fleischlos ernähren. Das Gefühl der Verstrickung in ein als maßlos und unmoralisch erlebtes System und damit die Thematisierung der eigenen Maßlosigkeit wecken den Bedarf nach Begrenzungen. Im Gesamtbild von Tierhaltung erklärt sich die Attraktivität der Museumslandwirtschaft auch aus ihrer Funktion als das Gegenbild zur Massentierhaltung. Entsprechende Begrenzungswünsche kommen auch im Wunsch oder im Vorsatz zum Ausdruck, den eigenen Fleischkonsum einzuschränken. Schuldzuweisungen und eigene Ohnmachtsbekundungen können ebenfalls helfen, das Gefühl der eigenen Verstrickung abzumildern. Bei Veganern und z.T. auch Vegetariern ist dagegen eine Tendenz festzustellen, die unmoralischen und ungesunden Aspekte der Tierhaltung zu betonen und damit die eigene, freiwillige Begrenzung zu begründen und positiv herauszustellen.

In der Auseinandersetzung um die Tierhaltung werden persönliche Zielkonflikte deutlich wahrgenommen, z.B. der Zielkonflikt zwischen einer preiswerten Versorgung und Forderungen nach verbesserten Tierschutzstandards. Es besteht auch ein Bewusstsein für den Widerspruch zwischen den Einstellungen zur Tierhaltung auf der einen Seite und dem Kauf- und Konsumverhalten auf der anderen. Hierfür werden unterschiedliche Argumente angeführt, wie z.B. Budgetrestriktionen, fehlende Verfügbarkeit von Fleisch mit höheren Tierwohlstandards, Scheu und Unlust vor der Verkomplizierung alltäglicher Kaufentscheidungen, fehlendes Wissen, fehlende Wirksamkeit des eigenen Handelns, fehlendes Verantwortungsgefühl für Missstände usw. Entsprechende Erklärungen verringern zwar das Unbehagen über das eigene Verhalten; sie beseitigen es aber nicht.
Je nachdem, in welchem Zusammenhang die Tierhaltung diskutiert wird (Schuldzuweisung, Beispiel für eine unmoralische Gesellschaft, eigener Fleischkonsum) kann die Beurteilung ein und desselben Teilnehmers unterschiedlich ausfallen. Es gibt eine erhebliche intrapersonelle Variabilität der geäußerten Einstellungen und der jeweils als wichtig erachteten Aspekte. Dies weist stark darauf hin, dass die Deutungen der Bilder vom jeweiligen Diskussionsrahmen abhängen.

\section{Ethische Einordnung}

Jede Wahrnehmung einer konkreten Tierhaltung wird beeinflusst von einem im Laufe des individuellen Lebens sich entwickelnden Spannungsfeld zwischen den Vorstellungen von „guter und richtiger“ bzw. „schlechter und falscher“ Tierhaltung. Die subjektive Vorstellung davon, was "gut und richtig“ bzw. „schlecht und falsch“ ist, resultiert aus den Bildern, die sich spontan einstellen, wenn die Tierhaltung in den Kategorien Mitgefühl, Gerechtigkeit und Respekt einmal als akzeptable und einmal als inakzeptable Variante beschrieben werden soll. Mitgefühl und Gerechtigkeit sind moralische Kategorien, die Empathie (Einfühlung in die Betroffenen) voraussetzen; Respekt bildet demgegenüber eine empathiefreie moralische Kategorie, die mit Wertschätzung korreliert. Werden Menschen aufgefordert, Bilder der landwirtschaftlichen Tierhaltung zu beschreiben, die sie als akzeptierbar empfinden, dann entstehen Bilder, die einen respektvollen Umgang mit den Tieren zeigen, die kein Mitleid auslösen und sich als „fairer Deal“ zwischen Mensch und Tier verstehen lassen. Bilder einer inakzeptablen Form landwirtschaftlicher Tierhaltung sind demgegenüber charakterisiert durch einen respektlosen Umgang mit dem Tier („Ausbeutung“, „Instrumentalisierung“), durch Mitleid oder durch eine Ungerechtigkeitsempfindung.

Während die Teilnehmer dieser Untersuchung die inakzeptablen Bilder von sich aus mit dem Begriff Massentierhaltung in Verbindung brachten, wurde Museumslandwirtschaft von den Autoren verwendet, um die Bilder der akzeptierten Tierhaltung begrifflich zusammenzufassen. Bezeichnet man die Pole des für die Nutztierhaltung relevanten Wertungsrahmens in diesem Sinne mit Museumslandwirtschaft und Massentierhaltung, dann wird verständlich, wie die beiden Bildgruppen bzw. die beiden Begriffe sich aus Einzelbildern zusammensetzen, die jeweils reale, mediale oder nur vorgestellte Erlebnisse des Beurteilenden darstellen. Auf der Seite der Museumslandwirtschaft dominieren schöne Bilder, weil 
Schönheit mit Wertschätzung und Wertschätzung mit Respekt assoziiert ist. Die Bilder der Massentierhaltung werden demgegenüber von abstoßenden Elementen geprägt (Dreck, Dunkelheit, Leid) und lassen Raum für düstere Phantasien.

Für die gesellschaftliche Debatte um die Zukunft der Nutztierhaltung kommt diesen Bildern bzw. Begriffen größte Wichtigkeit zu. Die Museumslandwirtschaft dient subjektiv als „Machbarkeitsnachweis“ einer ethisch sauberen Tierhaltung. Diese Bilder brauchen in der Praxis aber nicht eins zu eins realisiert werden (es ist kein Museumsbauernhof erforderlich). Notwendig ist vielmehr, dass die Bilder neuer Nutztierhaltungsformen in den drei angesprochenen Kategorien - Mitgefühl, Gerechtigkeit und Respekt ebenfalls als „akzeptierbar“ bewertet werden. Das ist dann der Fall, wenn sie als „fairer Deal“ zwischen Mensch und Tier verstanden werden können, weil ein „fairer Deal“ nicht nur Ungerechtigkeit, sondern auch Mitleid und Respektlosigkeit logisch ausschließt. Da sich in den vergangenen Jahrzehnten Schreckensphantasien zur Massentierhaltung verbreitet haben, erscheint es darüber hinaus unverzichtbar, Transparenz für den Bürger bzw. Verbraucher herzustellen („gläserne Produktion“), da nur so diesen sich spontan aufdrängenden Verdächtigungen entgegengewirkt werden kann.

\section{Zusammenfassende Einordnung}

Bei der Beurteilung und Einordnung der Ergebnisse der vorliegenden Studie als auch von qualitativen und quantitativen Befragungen im Allgemeinen ist $\mathrm{zu}$ berücksichtigen, dass die Bewusstmachung, die in solchen Befragungen stattfindet, im Alltag in der Regel nicht erfolgt. Entsprechend finden sich in den Interviews immer wieder Aussagen und „Bekenntnisse“, dass die als wichtig angesehene Tierschutzproblematik im Alltag kaum eine Rolle spielt. Die mentale Verfassung beim Kauf und Verzehr von Fleisch unterscheidet sich erheblich von der in der Untersuchungssituation. Unabhängig von der Bedeutung der Thematik im Alltag lassen sich für die Diskussion um die Wahrnehmung und Akzeptanz der Tierhaltung folgende Punkte herausstellen:

- Die Vorstellungen von der Tierhaltung und deren Akzeptanz sind stark von medialen Berichten, von Sehnsüchten als auch Schreckensphantasien und von Deutungsmustern beeinflusst. Das führt zu der Frage, inwieweit diese Vorstellungen den Verhältnissen in der Tierhaltung gerecht werden.
- Museumslandwirtschaft als akzeptierte und Massentierhaltung als nicht akzeptierte Form der Tierhaltung bilden eine weit verbreitete Bildkonfiguration. Die der Massentierhaltung zugeschriebene, angsteinflößende Maßlosigkeit findet einen Gegenpol in den als wohltuend erlebten Begrenzungen der Museumslandwirtschaft.

- Auch wenn die Grenzen der Umsetzbarkeit der Museumslandwirtschaft wahrgenommen werden, dienen die schönen Bilder des als fair empfundenen Deals zwischen Mensch und Tier als Maßstab einer zu entwickelnden ethisch und moralisch vertretbaren Tierhaltung.

- In den Interviews und Gruppendiskussionen gelingt es den Teilnehmern nicht, konkrete Vorstellungen von einer Tierhaltung zu entwickeln, die sowohl ihren Ansprüchen an Tierwohl und Umweltverträglichkeit, wie auch ihren Ansprüchen an Versorgung und Bezahlbarkeit entspricht. Eine Abwägung der mit einer „Museumslandwirtschaft“ verbundenen Konsequenzen erfolgt kaum.

- Reaktionen und Fragen nach der Beurteilung der Tierhaltung werden beeinflusst von der eigenen Verstrickung in das System. Bei Fleischessern können heftige Schuldzuweisungen und hohe moralischen Anforderungen an die Akteure der Wertschöpfungskette von der empfundenen Mitschuld ablenken.

- Argumentationen und geäußerte Einstellungen zur Tierhaltung variieren in Abhängigkeit vom Diskussionszusammenhang.

- Für eine Verbesserung der Akzeptanz der Tierhaltung sind Änderungen und Weiterentwicklungen hin zu tiergerechteren Verfahren notwendig, aber nicht hinreichend, wenn diese nicht auch zu einer Änderung der Bilder und Deutungsmuster führen. Kommunikation ist ein entscheidender Bestimmungsfaktor für die Entwicklung der Akzeptanz. Dabei ist zu beachten, dass eine Kommunikation, die als schön färbend wahrgenommen wird, zu einem weiteren Glaubwürdigkeitsverlust für den Sektor führen würde, weil sie die im Konzept der Massentierhaltung enthaltenen Deutungsmuster über die unmoralischen Handlungen der Akteure des Fleischsektors verstärkt.

- Bei Analyse der Marktreaktionen ist zu berücksichtigen, dass sich die mentale Verfassung in Kaufsituationen erheblich von der bei Befragungen unterscheidet. Spaltungen und Verdrängungen, die beim Kauf und Verzehr auftreten und die durch die Einbindung in bestimmte Situationen und 
Verwendungszusammenhänge gefördert werden, treten bei den Befragungen weniger stark auf.

\section{Literatur}

Bundesregierung (1971) Bundestagsdrucksache VI/ 2559 vom 07.09.1971. Entwurf eines Tierschutzgesetzes. http://dipbt.bundestag.de/doc/btd/06/025/ 0602559.pdf. Accessed 10 October 2017

Fitzek H (2010) Morphologische Beschreibung. In: Mey G, Mruck K. (eds.) Handbuch Qualitative Forschung in der Psychologie. VS Verlag für Sozialwissenschaften, Wiesbaden, pp 692-706
Lönneker J (2011) Die Wirkung von Qualitäten Gestalten im Wandel. In: Naderer G, Balzer E (eds.) Qualitative Marktforschung in Theorie und Praxis, 2nd edn. Gabler, Wiesbaden, pp 83-110

Melchers C, Ziems D (2001) Morphologische Marktpsychologie. Köln

Reik T (1983) Listening with the third ear: Macmillan. Farrar, Straus and Giroux, New York

Ziems D (2004) The morphological approach for unconscious consumer motivation research. J Advert Res 44(2): 210-224. https://doi.org/10.1017/ s0021849904040152 
Bürgerbewertungen unterschiedlicher Haltungssysteme von Milchkühen, Mastschweinen und Masthühnchen: Ein systematischer Vergleich

Sarah Kühl ${ }^{1}$, Winnie Isabel Sonntag ${ }^{1}$, Nina Gau $\aleph^{1}$, Birgit Gassler $^{1}$, Achim Spiller ${ }^{1}$

${ }^{1}$ Georg-August-Universität Göttingen, Department für Agrarökonomie und Rurale Entwicklung Marketing für Lebensmittel und Agrarprodukte sarah.kuehl@agr.uni-goettingen.de

\section{Einleitung}

Die Nutztierhaltung wird in Deutschland sehr differenziert wahrgenommen: Einerseits werden die Haltungsbedingen der Tiere vermehrt kritisiert und andererseits ist sie durch romantische Bilder aufgeladen (Kayser et al. 2012; Kayser und Spiller 2012; Isermeyer 2014). Zudem bestehen tierartenspezifische Unterschiede in der Wahrnehmung und Bewertung. Während die Milchviehhaltung im Vergleich zur Fleischwirtschaft eher positiv wahrgenommen wird (Albersmeier und Spiller 2010), wird bspw. die Hühnchenhaltung mit ihren hohen Tierzahlen und mechanisierten Abläufen negativ bewertet (Busch et al. 2015). Insgesamt erfährt die Nutztierhaltung, und dadurch auch der Konsum tierischer Produkte, eine zunehmende Skepsis und einen wachsenden Akzeptanzverlust in Teilen der Gesellschaft (Vanhonacker et al. 2014). Daher ist es wichtig zu ermitteln, welche Haltungssysteme bei welchen Tierarten akzeptiert oder abgelehnt werden und zu analysieren, worin diese Bewertungen begründet liegen.

Bisherige Studien konnten eine Präferenz der Bürger für Weidehaltung und Auslauf ins Freie für Nutztiere aufzeigen (Conner et al. 2008; Weinrich et al. 2014). Es existieren jedoch keine Studien, in denen Bewertungen hinsichtlich der verschiedenen modernen Haltungssysteme in systematischer Form erfasst wurden. Die vorliegende Untersuchung schließt diese Forschungslücke, indem deutsche Bürger in einer Online-Befragung verschiedene Haltungssysteme von Milchkühen, Mastschweinen und Mastgeflügel anhand von Bildern und Kurzbeschreibungen ohne Einbeziehung ökonomischer Abwägungen bewerten sollten. Die Ergebnisse dieser vergleichenden Bewertung ermöglichen Schlussfolgerungen für zukunftsweisende Haltungssysteme.

\section{Vorgehensweise und Auswertung}

Um Unterschiede in der Bewertung moderner Nutztierhaltungssysteme aufzuzeigen, wurde im Februar 2016 eine Online-Befragung mit 1.074 Probanden durchgeführt. Die Stichprobe war hinsichtlich Alter (ab 16 Jahren), Geschlecht und Einkommen repräsentativ für die deutsche Bevölkerung. Die Probanden beurteilten für Milchkühe, Mastschweine und Masthühnchen je 4 verschiedene Haltungssysteme (Stallhaltung, Außenklimastall, Stallhaltung mit Auslauf und Stallhaltung mit Weide im Sommer). Aufgrund des Umfangs der Fragestellung wurde der Fragebogen so geteilt, dass 358 Probanden die Milchviehhaltung bewerteten, 356 die Mastschweinehaltung und 360 die Masthühnchenhaltung. Eingesetzt wurden Bilder der jeweiligen Systeme und neutrale Kurzbeschreibungen ihrer wesentlichen Merkmale (vgl. Abb. 1-3). Die Bewertung der Haltungssysteme erfolgte anhand von literaturgestützten Statements (Conner et al. 2008), welche sich auf das Wohlbefinden der Tiere sowie auf die sensorischen Aspekte tierischer Produkte beziehen. Die Bewertung der vier Haltungssysteme erfolgte randomisiert, um Reihenfolgeeffekte zu vermeiden. Abschließend sollten die Probanden die gesehenen Haltungssysteme zusammenfassend bewerten. Die Datenauswertung erfolgte mittels uni- und bivariater Analysen in IBM SPSS Statistics 24. 


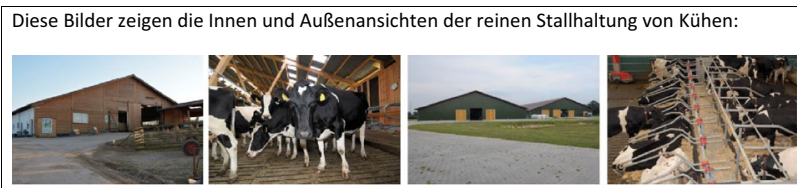

Die Kühe sind immer im Stall, dort können sie sich frei bewegen und sind nicht angebunden. Der Stall hat feste Wände mit Tür-, Tor- und Fensterflächen. Der Luftaustausch erfolgt über Lüftungsanlagen (z.B. Ventilatoren)

Quelle: (CBildagentur Landpixel

Diese Bilder zeigen die Innen und Außenansichten der reinen Stallhaltung von Kühen in Außenklimaställen

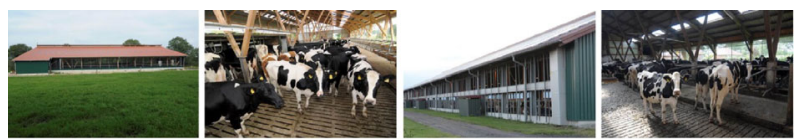

Die Kühe sind immer im Stall, dort können sie sich frei bewegen und sind nicht angebunden. Diese Stallform bietet im Gegensatz zu einem normalen Stall zusätzlich Kontakt zu Außenklima (natürliche Witterung: Sonnenschein, Kälte, Wind etc.) über große Öffnungen an der Front, offene Stallseitenwände oder einen offenen Giebel. Um die Tiere z.B. vor Wind zu schützen, stehen spezielle Windschutznetze oder steuerbare Jalousien an den offenen Wänden zur Verfügung. Die Temperatur und die Luftfeuchtigkeit sind im Stall ähnlich wie außerhalb des Stalls.

Quelle: (c) Bildagentur Landpixe

Diese Bilder zeigen die Innen- und Außenansichten der Stallhaltung von Kühen mit Zugang zu einem Auslauf (Laufhof), der den Tieren zusätzlich zur Verfügung steht:
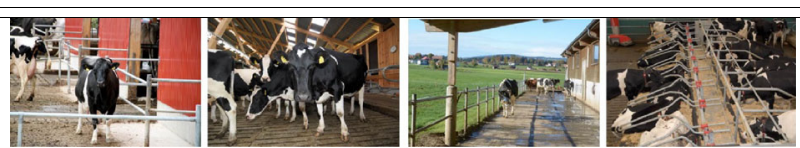

Die Kühe werden im Stall gehalten, dort können sie sich frei bewegen und sind nicht angebunden. Zusätzlich können sie nach eigenem Bedürfnis ganzjährig einen Auslauf (Laufhof) außerhalb des Stalls nutzen. Dieser Auslauf ist an den Stall angegliedert und hat meist einen betonierten Boden. Der Laufhof ist unter freiem Himmel oder teilweise überdacht und bietet den Tieren die Möglichkeit zum Orts- und Klimawechsel, sofern es die Witterung erlaubt.

Quelle: (c) Bildagentur Landpixel

Diese Bilder zeigen die Innen- und Außenansichten der Stallhaltung von Kühen mit Zugang auf eine Weide bzw. einen Naturboden, der den Tieren zusätzlich zur Verfügung steht:
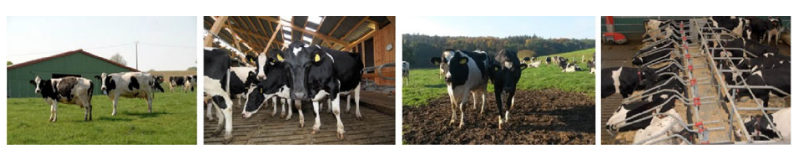

Die Kühe werden im Stall gehalten, dort können sie sich frei bewegen und sind nicht angebunden. Zusätzlich haben sie für einen bestimmten Zeitraum des Jahres, sofern es die Witterung erlaubt, Zugang auf eine Weide bzw. einen Naturboden. Der Zugang auf die Weide bzw. den Naturboden bietet die Möglichkeit zum Orts- und Klimawechsel.

Quelle: () Nina Gaus

Abb. 1 Bewertungsgrundlagen für verschiedene Haltungssysteme in der Online-Befragung zur Tierart „Kuh“

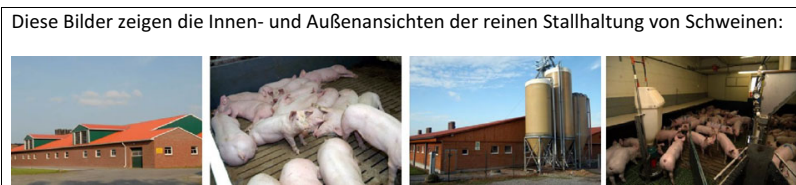

Die Schweine sind immer im Stall, dort können sie sich frei bewegen und sind mit anderen Schweinen zusammen in einer Gruppenbucht. Der Stall hat feste Wände mit geschlossenen Tür-, Tor- und Fensterflächen. Lüftungs- und Klimageräte sorgen für ein geregeltes Raumklima, unabhängig von der Temperatur und der Luftfeuchtigkeit draußen.

Diese Bilder zeigen die Innen- und Außenansichten der reinen Stallhaltung von Schweinen in Außenklimaställen

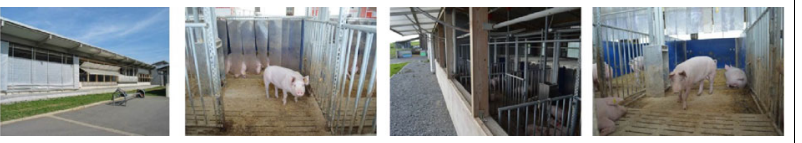

Die Schweine sind immer im Stall, dort können sie sich frei bewegen und sind mit anderen Schweinen zusammen in einer Gruppenbucht. Diese Stallform bietet im Gegensatz zu einem normalen Stall zusätzlich Kontakt zu Außenklima (natürliche Witterung: Sonnenschein, Kälte, Wind etc.) über offene Seitenwände. Um die Tiere z.B. vor Wind zu schützen, stehen spezielle Windschutznetze oder steuerbare Jalousien an den offenen Wänden zur Verfügung. Die Liege- und Ruhezonen sind wärmegedämmt oder eingestreut. Die Schweine können im Stall einen beliebigen Klimabereich aufsuchen.

Quelle: (c) Bildungs und Wissenszentrum Boxberg Schweinehaltung, Schweinezucht (Landesanstalt für Schweinezucht LSZ)

Diese Bilder zeigen die Innen- und Außenansichten der Stallhaltung von Schweinen mit Zugang zu einem Auslauf, der den Tieren zusätzlich zur Verfügung steht:
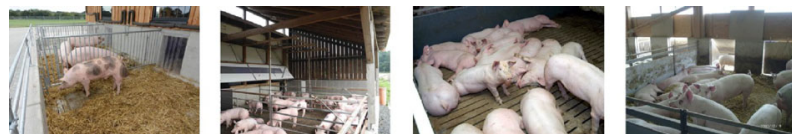

Die Schweine werden im Stall gehalten, dort können sie sich frei bewegen und sind mit anderen Schweinen zusammen in einer Gruppenbucht. Zusätzlich können sie nach eigenem Bedürfnis ganzjährig einen Auslauf außerhalb des Stalls nutzen. Dieser Auslauf ist an den Stall angegliedert und ist meist betoniert und nicht zwingend mit Stroh eingestreut. Der Auslauf ist unter freiem Himmel oder teilweise überdacht und bietet den Tieren die Möglichkeit zum Orts- und Klimawechsel, sofern es die Witterung erlaubt.

Quelle: (c) Bildagentur Landpixel, (c) KTBL, Stephan Fritsche

Diese Bilder zeigen die Haltung von Schweinen in Hütten mit Zugang auf eine Wiese bzw. einen Naturboden:
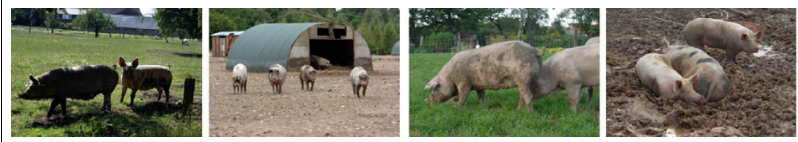

Die Schweine werden für eine gewisse Zeit im Stall gehalten, dort können sie sich frei bewegen und sind mit anderen Schweinen zusammen in einer Gruppenbucht. Außerdem leben Sie für einen bestimmten Zeitraum des Jahres im Freien auf einer umzäunten Wiese bzw. einem Naturboden. Dort stehen ihnen zum Schutz vor der Witterung Hütten zur Verfügung. Sie können sich auf der Wiese bzw. dem Naturboden zusammen mit anderen Schweinen nach ihrem eigenem Bedürfnis frei bewegen.

Quelle: (c) Bildagentur Landpixel

Abb. 2 Bewertungsgrundlagen für verschiedene Haltungssysteme in der Online-Befragung zur Tierart „Schwein“ 


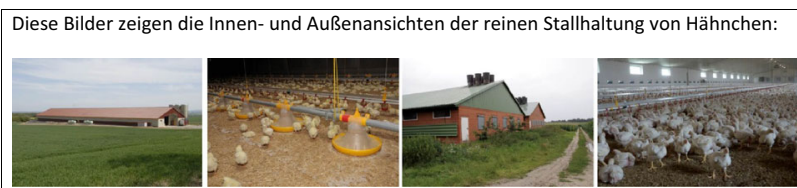

Die Hähnchen sind immer im Stall, dort werden sie auf Einstreu gehalten und können sich frei bewegen. Der Stall hat feste Wände mit geschlossenen Tür-, Tor- und Fensterflächen. Lüftungs- und Klimageräte sorgen für ein geregeltes Raumklima, unabhängig von der

Temperatur und der Luftfeuchtigkeit draußen. Quelle: @ Bildagentur Landpixel

Diese Bilder zeigen die Innen- und Außenansichten der reinen Stallhaltung von Hähnchen in Außenklimaställen

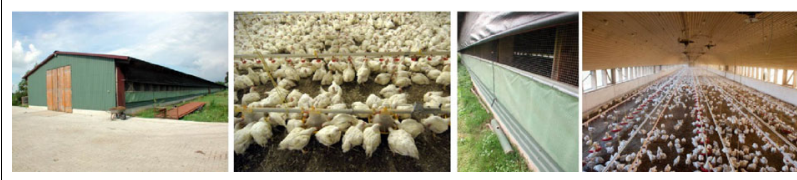

Die Hähnchen sind immer im Stall, dort werden sie auf Einstreu gehalten und können sich frei bewegen. Diese Stallform bietet im Gegensatz zu einem normalen Stall zusätzlich Kontakt zu Außenklima (natürliche Witterung: Sonnenschein, Kälte, Wind etc.) über offene Seitenwände. Um die Tiere z.B. vor Wind zu schützen, stehen spezielle Windschutznetze oder steuerbare Jalousien an den offenen Wänden zur Verfügung. Ventilatoren sorgen zusätzlich für den Luftaustausch. Quelle: @ Wiesenhof Privathof, Hof Goebl

Diese Bilder zeigen die Innen- und Außenansichten der Stallhaltung von Hähnchen mit Zugang zu einem Auslauf (Kaltscharrraum), der den Tieren zusätzlich zur Verfügung steht:
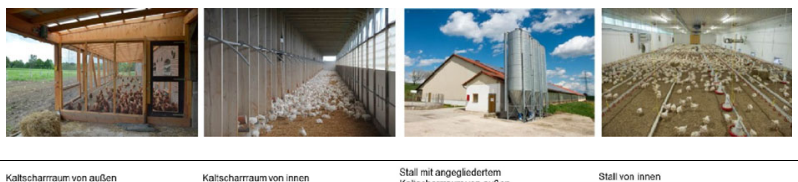

karscharraumven auser
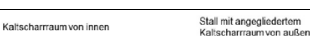

Stall ven iner

Die Hähnchen werden im Stall auf Einstreu gehalten und können sich dort frei bewegen. Zusätzlich können sie nach eigenem Bedürfnis ganzjährig einen Auslauf (Kaltscharrraum) außerhalb des Stalls nutzen. Ein Kaltscharrraum ist meist an die Längsseite des Stalls angegliedert, ist betoniert und eingestreut. Dieser Auslauf ist ein überdachter und engmaschig umzäunter Außenbereich, der den Tieren die Möglichkeit zum Orts- und

Klimawechsel bietet, sofern es die Witterung erlaubt. (C) Bildagentur Landpixel,

(c) Wiesenhof Privathof, Hof Attenberger, Hof Altinger, Hof Pirzer

Diese Bilder zeigen die Innen- und Außenansichten der Stallhaltung von Hähnchen mit

Zugang auf eine Grünfläche bzw. einen Naturboden, der den Tieren zusätzlich zur Verfügung steht:
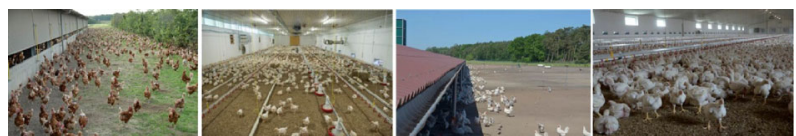

Die Hähnchen werden im Stall auf Einstreu gehalten und können sich dort frei bewegen. Zusätzlich haben sie ab einem gewissen Alter Zugang auf eine Grünfläche bzw. einen Naturboden. Der Zugang auf die Grünfläche bzw. den Naturboden besteht für einen bestimmten Zeitraum des Jahres und wenn es die klimatischen Bedingungen erlauben. Die Hähnchen haben tagsüber die Möglichkeit zum Orts- und Klimawechsel.

Quelle: ( ) Bildagentur Landpixel, @ W Wiesenhof Privathof, Hof Pirzer

Abb. 3 Bewertungsgrundlagen für verschiedene Haltungssysteme in der Online-Befragung zur Tierart „Hühnchen“

\section{Ergebnisse}

\subsection{Stichprobenbeschreibung}

Das Geschlechterverhältnis in der Stichprobe ist mit $51,6 \%$ weiblichen $\mathrm{zu} 48,4 \%$ männlichen Teilnehmenden ausgeglichen und bevölkerungsrepräsentativ. Unter den Befragten ergibt sich eine Altersspanne von 16 bis 81 Jahren mit einem Durchschnittsalter von 47 Jahren. Die vorliegende Stichprobe entspricht hinsichtlich Geschlecht, Alter und Einkommen annähernd dem deutschen Bevölkerungsdurchschnitt. 6,2\% der Probanden waren Vegetarier, wobei der Anteil der Frauen (79,1\%) unter den Vegetariern überwog.

\subsection{Tierartübergreifende Wahrnehmungen der Haltungssysteme}

3.2.1 Milchviehhaltung Von allen Haltungssystemen wurde der Stall mit Weidezugang am positivsten beurteilt; gefolgt vom Stall mit Auslauf und dem Außenklimastall. Tabelle 1 zeigt, dass $80 \%$ der Befragten zustimmten, dass die Tiere mit Weidezugang gesund leben, während dies für den Außenklimastall von 31 \% der Probanden und für den Stall mit Auslauf von $38 \%$ der Probanden zustimmend bewertet wurde (zwischen letzteren keine signifikanten Unterschiede). 
Tab. 1 Bewertung der Haltungssysteme für Milchkühe anhand ausgewählter Kriterien

\begin{tabular}{|c|c|c|c|c|c|c|c|c|c|c|c|c|}
\hline \multirow[t]{2}{*}{ Statements } & \multicolumn{3}{|c|}{ Stall } & \multicolumn{3}{|c|}{ Außenklimab } & \multicolumn{3}{|c|}{ Stall mit Auslauf ${ }^{\mathrm{C}}$} & \multicolumn{3}{|c|}{ Stall mit Weide ${ }^{d}$} \\
\hline & -- & $+1-$ & ++ & -- & $+1-$ & ++ & -- & $+1-$ & ++ & -- & $+1-$ & ++ \\
\hline \multirow[t]{2}{*}{ Die Tiere leben hier gesund.**** } & $61 \%$ & $31 \%$ & $8 \%$ & $26 \%$ & $43 \%$ & $31 \%$ & $19 \%$ & $44 \%$ & $38 \%$ & $3 \%$ & $18 \%$ & $80 \%$ \\
\hline & \multicolumn{3}{|c|}{$2,25^{\mathrm{bcd}}(0,96)$} & \multicolumn{3}{|c|}{$3,06^{\text {ad }}(0,94)$} & \multicolumn{3}{|c|}{$3,25^{\mathrm{ad}}(0,98)$} & \multicolumn{3}{|c|}{$4,13^{\mathrm{abc}}(0,83)$} \\
\hline \multirow[t]{2}{*}{ Hier fehlt es den Kühen an nichts. ${ }^{* * *}$} & $75 \%$ & $19 \%$ & $6 \%$ & $58 \%$ & $29 \%$ & $13 \%$ & $40 \%$ & $33 \%$ & $26 \%$ & $6 \%$ & $24 \%$ & $70 \%$ \\
\hline & \multicolumn{3}{|c|}{$2,01^{\text {bcd }}(0.88)$} & \multicolumn{3}{|c|}{$2,41^{\text {acd }}(1,00)$} & \multicolumn{3}{|c|}{$2,86^{\text {abd }}(1,12)$} & \multicolumn{3}{|c|}{$3,94^{\mathrm{abc}}(0,94)$} \\
\hline \multirow[t]{2}{*}{ Hier bekommen die Kühe ausreichend Tageslicht. ${ }^{* * *}$} & $75 \%$ & $18 \%$ & $7 \%$ & $24 \%$ & $38 \%$ & $38 \%$ & $5 \%$ & $23 \%$ & $72 \%$ & $2 \%$ & $14 \%$ & $84 \%$ \\
\hline & \multicolumn{3}{|c|}{$2,04^{\text {bcd }}(0,93)$} & \multicolumn{3}{|c|}{$3,15^{\text {acd }}(1,00)$} & \multicolumn{3}{|c|}{$3,88^{\mathrm{abd}}(0,85)$} & \multicolumn{3}{|c|}{$4,25^{\mathrm{abc}}(0,78)$} \\
\hline \multirow[t]{2}{*}{ Hier bekommen die Kühe ausreichend Frischluft. ${ }^{* * *}$} & $52 \%$ & $34 \%$ & $15 \%$ & $15 \%$ & $28 \%$ & $56 \%$ & $4 \%$ & $20 \%$ & $77 \%$ & & $9 \%$ & $89 \%$ \\
\hline & \multicolumn{3}{|c|}{$2,47^{\text {bcd }}(1,01)$} & \multicolumn{3}{|c|}{$3,50^{\text {acd }}(0,96)$} & \multicolumn{3}{|c|}{$3,96^{\text {abd }}(0.79)$} & \multicolumn{3}{|c|}{$4,32^{\mathrm{abc}}(0,73)$} \\
\hline \multirow{2}{*}{$\begin{array}{l}\text { Die Tiere können hier ihr natürliches } \\
\text { Verhalten voll ausleben.*** }\end{array}$} & $86 \%$ & $10 \%$ & $4 \%$ & $66 \%$ & $23 \%$ & $11 \%$ & $48 \%$ & $28 \%$ & $24 \%$ & $2 \%$ & $13 \%$ & $85 \%$ \\
\hline & \multicolumn{3}{|c|}{$1,73^{\text {bcd }}(0,82)$} & \multicolumn{3}{|c|}{$2,23^{\text {acd }}(1,02)$} & \multicolumn{3}{|c|}{$2.69^{\text {abd }}(1,15)$} & \multicolumn{3}{|c|}{$3,87^{\mathrm{abc}}(0,95)$} \\
\hline \multirow[t]{2}{*}{ Milch von diesen Tieren schmeckt gut. ${ }^{* * *}$} & $31 \%$ & $42 \%$ & $27 \%$ & $19 \%$ & $43 \%$ & $38 \%$ & $12 \%$ & $35 \%$ & $52 \%$ & $2 \%$ & $13 \%$ & $85 \%$ \\
\hline & \multicolumn{3}{|c|}{$2,89^{\text {bcd }}(0,97)$} & \multicolumn{3}{|c|}{$3,18^{\text {acd }}(0,93)$} & \multicolumn{3}{|c|}{$3,50^{\mathrm{abd}}(0.91)$} & \multicolumn{3}{|c|}{$4,15^{\mathrm{abc}}(0,75)$} \\
\hline Dem Landwirt ist das Wohl seiner Tiere & $57 \%$ & $34 \%$ & $10 \%$ & $27 \%$ & $46 \%$ & $27 \%$ & $18 \%$ & $42 \%$ & $40 \%$ & & $13 \%$ & $85 \%$ \\
\hline wichtig, wenn er sie so hält.**** & $2,31^{b c}$ & ${ }^{d}(0,9$ & & $2,97^{\mathrm{a}}$ & $d(1,0$ & & $3,29^{a}$ & d $(1,0$ & & $4,18^{a b c}$ & ${ }^{c}(0,7$ & \\
\hline
\end{tabular}

Die auf einer 5-stufigen Skala bewerteten Statements wurden wie folgt zusammengefasst:

- „Stimme überhaupt nicht zu (1)“ und „Stimme nicht zu (2)“; +- „Teils, teils (3)“; ++ „Stimme zu (4)“ und „Stimme voll und ganz zu (5)“

Angegeben sind zusätzlich jeweils der Mittelwert und die Standardabweichung in Klammern

Signifikanzniveau für Unterschiede zwischen den Haltungssystemen: ${ }^{* * *} \mathrm{p} \leq 0.000$

a,b,c,d Signifikante Unterschiede $(p \leq 0.05)$ zwischen den einzelnen Haltungssystemen, die mittels post hoc Tests ermittelt wurden

Die reine Stallhaltung wird skeptisch bewertet: Drei von vier Probanden lehnten die Aussage ab, dass es Milchkühen im Stall an nichts fehlt. Vor allem hinsichtlich des Mangels an ausreichendem Tageslicht wurde die Stallhaltung kritisch beurteilt. Erst bei Weidegang waren $70 \%$ der Befragten überzeugt, dass es den Kühen an nichts fehlt. Zusätzlich waren 7 von 10 Probanden nur bei einem Auslauf ins Freie der Meinung, dass den Tieren ausreichend Tageslicht und Frischluft zur Verfügung stehen.

Die ausschließliche Stallhaltung wurde vor allem hinsichtlich der Möglichkeiten der Kühe ihr natürliches Verhalten auszuleben, kritisch beurteilt, denn $86 \%$ der Befragten sahen diese nicht gegeben. Die Tierwohlbewertung strahlt auf die intrinsischen Produktmerkmale ab: Weniger als ein Drittel der Befragten schätzte Milch von Kühen aus reiner Stallhaltung als gut im Geschmack ein, während bei Milch von Kühen mit Weidezugang der Geschmack überaus positiv bewertet wurde. Auffallend hoch war auch die Zustimmung zum Statement, dass Landwirten das Wohl der Tiere wichtig ist, wenn den Tieren Weidezugang ermöglicht wird. Bei reiner Stallhaltung waren davon nur $10 \%$ der Probanden überzeugt.

\subsubsection{Mastschweinehaltung}

Der Stall mit Weidezugang wurde auch in der Mastschweinehaltung am positivsten bewertet, die reine Stallhaltung deutlich negativer. Im Gegensatz zur differenzierten Wahrnehmung des Außenklimastalls und des Stalls mit Auslauf bei der Milchvieh- und Geflügelhaltung zeigten sich jedoch kaum signifikante Unterschiede in der Bewertung dieser beiden Haltungssysteme für Mastschweine (Tab. 2). $92 \%$ der Befragten waren der Meinung, dass Schweine, die Zugang zu Weideflächen haben, gesund leben, während dies für den Außenklimastall und den Stall mit Auslauf nur ein Drittel der Probanden zustimmend beantwortete; für den dargestellten Stall lehnten zwei Drittel der Befragten dieses Statement ab. Kaum ein Befragter vertrat die Meinung, dass es diesen Tieren an nichts fehlt. Die Möglichkeiten natürliches Verhalten auszuleben und ausreichend Zugang zu Tageslicht und Frischluft, wurden für die Stallhaltung am negativsten bewertet. Auch beim Außenklimastall und dem Stall mit Auslauf war rund die Hälfte der Befragten der Ansicht, dass die Tiere in beiden Systemen ihr natürliches Verhalten nicht ausleben können. Erst mit Weidezugang stimmten 90 \% der Befragten dieser Aussage zu. 
Tab. 2 Bewertung der Haltungssysteme für Mastschweine anhand ausgewählter Kriterien

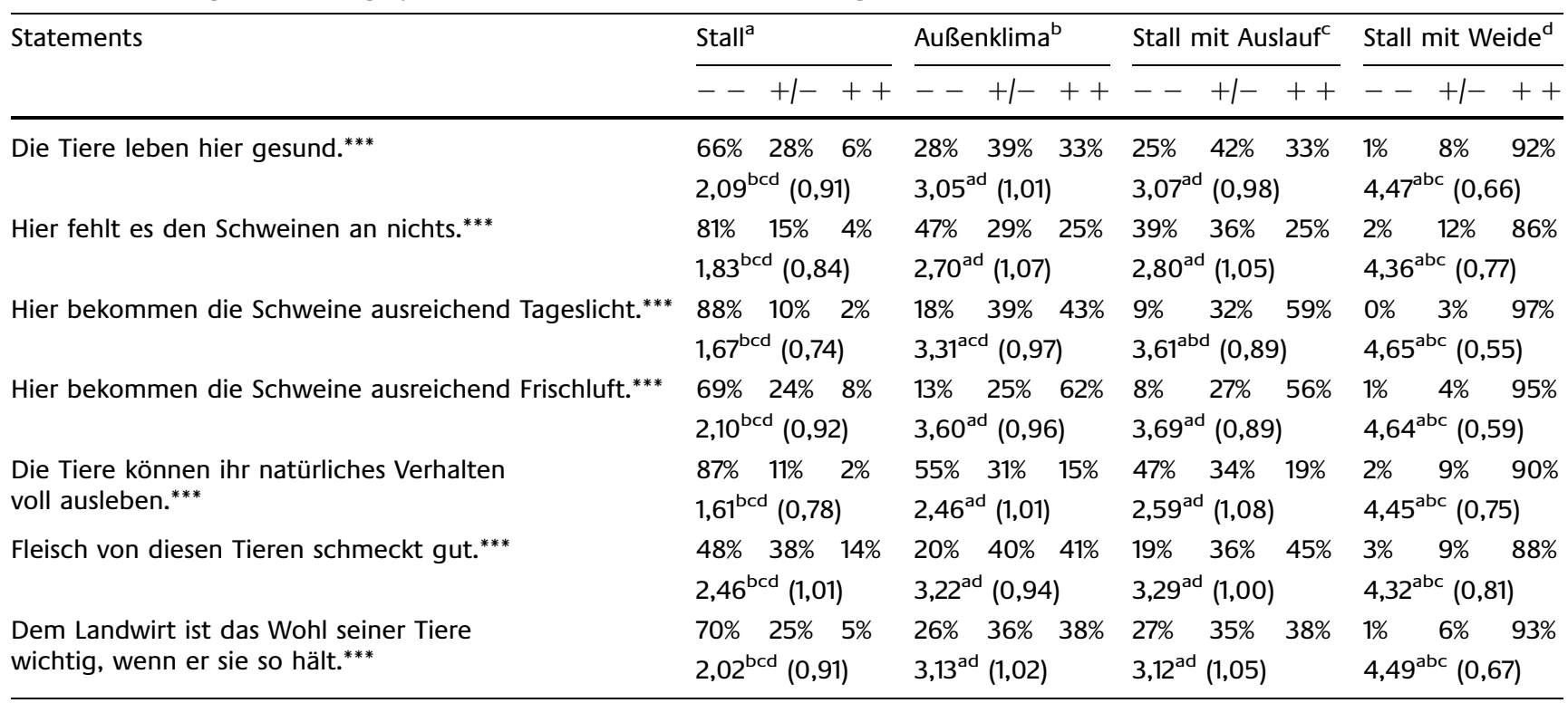

Die auf einer 5-stufigen Skala bewerteten Statements wurden wie folgt zusammengefasst:

- „Stimme überhaupt nicht zu(1)“ und „Stimme nicht zu(2)“; +- „Teils, teils(3)“; ++ „Stimme zu(4)“ und „Stimme voll und ganz zu(5)“ Angegeben sind zusätzlich jeweils der Mittelwert und die Standardabweichung in Klammern Signifikanzniveau für Unterschiede zwischen den Haltungssystemen: ${ }^{* * *} \mathrm{p} \leq 0.000$

$a, b, c, d$ Signifikante Unterschiede $(p \leq 0.05)$ zwischen den einzelnen Haltungssystemen, die mittels post hoc Tests ermittelt wurden

Bei Schweinen polarisiert im Vergleich zu Kühen und Hühnern die Geschmacksbewertung besonders deutlich, stark negativ für die Stallhaltung, sehr positiv für die Freilandhaltung. Die Ansicht, dass Landwirten das Wohl der Tiere nicht wichtig sei, wenn die Tiere im Stall gehalten werden, vertraten 7 von 10 Probanden. Außenklimastall und Stall mit Auslauf wurden positiver bewertet, aber nur mit Weidezugang wurde den Landwirten überwiegend zuerkannt, dass ihnen das Wohl ihrer Tiere wichtig ist.

\subsubsection{Masthühnchenhaltung}

Auch für die Masthühnchenhaltung wurde die Stallhaltung mit Weidezugang in allen Kriterien am positivsten beurteilt. Tabelle 3 verdeutlicht ähnlich wie bei Kühen, dass ein Stall mit Auslauf positiver bewertet wird als ein Außenklimastall, und dieser wiederum positiver als die reine Stallhaltung.

Tab. 3 Bewertung der Haltungssysteme für Masthühnchen anhand ausgewählter Kriterien

\begin{tabular}{|c|c|c|c|c|}
\hline \multirow[t]{2}{*}{ Statements } & Stall ${ }^{a}$ & Außenklimab & Stall mit Auslauf ${ }^{\mathrm{C}}$ & Stall mit Weide \\
\hline & $--+1-++$ & $--+1-++$ & $--+1-\quad++$ & $--+1-++$ \\
\hline \multirow[t]{2}{*}{ Die Tiere leben hier gesund.*** } & $70 \% \quad 23 \% \quad 7 \%$ & $47 \% \quad 38 \% \quad 16 \%$ & $30 \% \quad 43 \% \quad 27 \%$ & $11 \% \quad 29 \% \quad 59 \%$ \\
\hline & $2,08^{\text {bcd }}(0,95)$ & $2,56^{\text {acd }}(0,98)$ & $2,94^{\mathrm{abd}}(0,98)$ & $3,70^{\mathrm{abc}}(0,89)$ \\
\hline \multirow[t]{2}{*}{ Die Tiere können ihr natürliches Verhalten voll ausleben. ${ }^{* * *}$} & $85 \% \quad 10 \% \quad 5 \%$ & $73 \% \quad 19 \% \quad 8 \%$ & $49 \% \quad 36 \% \quad 15 \%$ & $11 \% \quad 26 \% \quad 62 \%$ \\
\hline & $1,69^{\mathrm{bcd}}(0,87)$ & $2,07^{\text {acd }}(0,94)$ & $2,55^{\mathrm{abd}}(1,03)$ & $3,56^{\mathrm{abc}}(1,01)$ \\
\hline \multirow[t]{2}{*}{ Hier fehlt es den Hähnchen an nichts. ${ }^{* * *}$} & $82 \% \quad 11 \% \quad 6 \%$ & $65 \% \quad 25 \% \quad 22 \%$ & $47 \% \quad 38 \% \quad 16 \%$ & $3 \% \quad 20 \% \quad 76 \%$ \\
\hline & $1,88^{\mathrm{bcd}}(0,91)$ & $2,29^{\text {acd }}(0,96)$ & $2,62^{\mathrm{abd}}(1,00)$ & $3,47^{\mathrm{abc}}(1,03)$ \\
\hline \multirow[t]{2}{*}{ Hier bekommen die Hähnchen ausreichend Tageslicht.**** } & $87 \% \quad 9 \% \quad 4 \%$ & $35 \% \quad 34 \% \quad 31 \%$ & $21 \% \quad 39 \% \quad 39 \%$ & $6 \% \quad 17 \% \quad 77 \%$ \\
\hline & $1,70^{\mathrm{bcd}}(0,83)$ & $2,91^{\text {acd }}(1,07)$ & $3,24^{\mathrm{abd}}(0,98)$ & $4,05^{\mathrm{abc}}(0,85)$ \\
\hline
\end{tabular}


Tab. 3 continued

\begin{tabular}{|c|c|c|c|c|}
\hline \multirow[t]{2}{*}{ Statements } & Stall ${ }^{a}$ & Außenklima ${ }^{\mathrm{b}}$ & $\begin{array}{l}\text { Stall mit } \\
\text { Auslauf }\end{array}$ & Stall mit Weide \\
\hline & $--+1-++$ & $--+1-++$ & $--+1-++$ & $--+1-++$ \\
\hline Hier bekommen die Hähnchen ausreichend Frischluft. ${ }^{* * *}$ & $\begin{array}{l}63 \% \quad 26 \% \quad 10 \% \\
2,20^{\text {bcd }}(1,00)\end{array}$ & $\begin{array}{l}21 \% \quad 35 \% \quad 44 \% \\
3,23^{\text {ad }}(1,07)\end{array}$ & $\begin{array}{l}18 \% \quad 34 \% \quad 48 \% \\
3,38^{\text {ad }}(0,99)\end{array}$ & $\begin{array}{l}9 \% \quad 22 \% \quad 69 \% \\
4,09^{\text {abc }}(0,82)\end{array}$ \\
\hline Fleisch von diesen Tieren schmeckt gut. ${ }^{* * *}$ & $\begin{array}{l}48 \% 34 \% \quad 18 \% \\
2,53^{\text {bcd }}(1,04)\end{array}$ & $\begin{array}{l}35 \% \quad 41 \% \quad 25 \% \\
2,82^{\text {acd }}(0,98)\end{array}$ & $\begin{array}{l}22 \% \quad 43 \% \quad 34 \% \\
3,19^{\text {abd }}(0,96)\end{array}$ & $\begin{array}{l}15 \% \quad 29 \% \quad 56 \% \\
3,78^{\text {abc }}(0,89)\end{array}$ \\
\hline $\begin{array}{l}\text { Dem Landwirt ist das Wohl seiner Tiere wichtig, wenn er sie so } \\
\text { hält.*** }\end{array}$ & $\begin{array}{l}76 \% \quad 17 \% \quad 8 \% \\
1,96^{\text {bcd }}(0,96)\end{array}$ & $\begin{array}{l}47 \% 36 \% \quad 17 \% \\
2,58^{\text {acd }}(1,01)\end{array}$ & $\begin{array}{l}26 \% \quad 43 \% \quad 31 \% \\
3,03^{\text {abd }}(1,02)\end{array}$ & $\begin{array}{l}11 \% 21 \% \quad 68 \% \\
3,77^{\mathrm{abc}}(0,95)\end{array}$ \\
\hline
\end{tabular}

Die auf einer 5-stufigen Skala bewerteten Statements wurden wie folgt zusammengefasst:

- „Stimme überhaupt nicht zu(1)“ und „Stimme nicht zu(2)“; +- „Teils, teils(3)“; ++ „Stimme zu(4)“ und „Stimme voll und ganz zu(5)“ Angegeben sind zusätzlich jeweils der Mittelwert und die Standardabweichung in Klammern

Signifikanzniveau für Unterschiede zwischen den Haltungssystemen: ${ }^{* * *} \mathrm{p} \leq 0.000$

a,b,c,d Signifikante Unterschiede $(p \leq 0.05)$ zwischen den einzelnen Haltungssystemen, die mittels post hoc Tests ermittelt wurden

Lediglich 7 \% der Befragten stimmten zu, dass Tiere im Stall ein gesundes Leben führen. Die Bewertung des Stalls mit Weide fiel mit 6 von 10 zustimmenden Probanden weniger positiv aus als für die Milchviehund Mastschweinehaltung. Bei Stallhaltung sahen 85 \% der Befragten keine Möglichkeit für die Tiere ihr natürliches Verhalten ausleben zu können; für den Außenklimastall sahen dies rund drei Viertel der Probanden als nicht gegeben an und für den Stall mit Auslauf rund die Hälfte der Probanden. Nur mit Weidezugang waren 3 von 4 Probanden überzeugt, dass es den Tieren an nichts fehlt. Bei Stallsystemen wurde ausreichendes Tageslicht und Frischluft angezweifelt; während beides für den Außenklimastall positiver bewertet wurde.

Etwa die Hälfte der Befragten lehnte zudem die Aussage ab, dass Hühnchenfleisch von Tieren aus Stallhaltung gut schmeckt. $76 \%$ der Probanden waren der Ansicht, dass Landwirten das Wohl der Tiere nicht wichtig sei, wenn sie im Stall gehalten werden. Beim Außenklimastall traf dies für die Hälfte der Befragten zu.

\subsubsection{Vergleichende Darstellung zwischen den Tierarten}

Über alle Tierarten hinweg zeigt sich damit eine klare Präferenzreihung der Haltungssysteme, es gibt aber auch tierartenspezifische Unterschiede. Die reine Stallhaltung wird insbesondere hinsichtlich ausreichend Tageslicht und Frischluft für Milchkühe positiver bewertet als für Masthühnchen- und Mastschweine. Der Außenklimastall wird vor allem in Bezug auf das Wohlbefinden von Mastschweinen signifikant positiver bewertet, während für Milchkühe und Masthühnchen erst der Auslauf die Zustimmung erhöht; für Mastschweine kann ein Auslauf am Stall die Bewertung im Vergleich zum Außenklimastall nicht weiter verbessern. Bei Stallhaltung mit Weidegang stimmten die Probanden über alle Tierarten hinweg am stärksten zu, dass die Tiere gesund leben, ausreichend Tageslicht bekommen und ihr natürliches Verhalten ausleben können. Allerdings war diese Zustimmung für Mastschweine signifikant höher als für Milchkühe und Masthühnchen.

Bei der Erwartung an geschmackliche Eigenschaften der tierischen Produkte zeigt sich ein einheitliches Bild: So werden Produkte von Tieren, die im Stall gehalten werden, negativer bewertet als Produkte von Tieren aus Haltungssystemen mit Zugang ins Freie. Allerdings ist die Zustimmung, dass Hühnchenfleisch aus Haltungssystemen mit Zugang zu einer Grünfläche gut schmeckt, signifikant geringer als für Fleisch von Mastschweinen und Milch von Kühen mit Zugang zu einer Weide.

\section{Schlussfolgerungen}

Die Ergebnisse zeigen deutlich, dass die Gesellschaft die reine Stallhaltung für Nutztiere ablehnt. Eine gewisse Skepsis war auf Basis der bisherigen Literatur zu erwarten (Kayser et al. 2012; Busch et al. 2015), die Stärke der Ablehnung ist jedoch frappierend. Auch Außenklimaställe können die gesellschaftliche Akzeptanz nur bedingt erhöhen. Das kann ein Hinweis darauf sein, dass die Verbesserungen gegenüber der reinen Stallhaltung zwar wahrgenommen, aber 
als noch nicht ausreichend eingeschätzt werden. Lediglich für Mastschweine konnte diese Haltungsform eine Akzeptanz von über 20 \% erzielen.

Insbesondere hinsichtlich der Natürlichkeit des Haltungssystems schneidet die reine Stallhaltung schlecht ab. Die Probanden vermuten, dass die Tiere hier nicht ausreichend Tageslicht und Frischluft bekommen und ihr natürliches Verhalten nicht ausleben können. Eine natürliche Haltungsumwelt ist offensichtlich maßgeblich für eine positive Bewertung und ethische Vertretbarkeit der Nutztierhaltung (Lassen et al. 2006; Dawkins und Bonney 2008; Boogaard et al. 2006). Dabei finden sich auch Unterschiede zwischen den Tierarten. Die reine Stallhaltung wird in der Milchviehhaltung bspw. positiver bewertet als für Mastschweine und Masthühnchen. Trotz der positiveren Bewertung bzgl. Frischluftkontakt und Wohlbefinden findet aber auch für Milchkühe die reine Stallhaltung kaum Akzeptanz.

Der Außenklimastall wird insbesondere für Mastschweine bzgl. Tageslicht- und Frischluftkontakt, aber auch bzgl. des Wohlbefindens der Tiere deutlich positiver bewertet als die geschlossene Stallhaltung. Die Akzeptanz bleibt jedoch niedrig. In der Masthühnchenhaltung hat der Außenklimastall kaum einen positiven Effekt auf die Akzeptanz. Generell werden alle Haltungssysteme für Masthühnchen am negativsten bewertet. Dies könnte daraus resultieren, dass sich Bürger von der großen Anzahl der Tiere meist überfordert fühlen und laut Studien bspw. eine Reduzierung der Bestandsgröße gar nicht wahrnehmen (Busch et al. 2015).

Damit die Nutztierhaltung langfristig gesellschaftlich akzeptiert wird, ist der Zugang ins Freie notwendig. Für alle Tierarten zeigt sich eine klare Präferenz für Haltungssysteme, die den Tieren Zugang ins Freie ermöglichen. Dabei wird die Weidehaltung am besten bewertet, was sich auch in anderen Studien des SocialLab-Projektes zeigt (wie z.B. von Rovers et al. in diesem Heft). Diese hohe Akzeptanz entspricht zudem den Ergebnissen anderer Autoren (Cardoso et al. 2016; Sonntag et al. 2017). Aber auch ein Auslauf erhöht die Akzeptanz deutlich. Etwa die Hälfte aller Probanden akzeptiert die Stallhaltung, wenn die Tiere zumindest Zugang zu einem Auslauf haben - der Anteil der Probanden, die dieses System nicht akzeptieren, sinkt auf unter $20 \%$ bei allen Nutztieren.

Zudem werden Haltungssysteme mit Auslauf oder Weide mit positiven Auswirkungen auf den Geschmack der Produkte assoziiert. Dies ist ein wichtiges Ergebnis für die Vermarktung von Produkten aus diesen Haltungssystemen und bestätigt die positiven Wechselbeziehungen zwischen der Wahrnehmung des altruistischen Merkmals Tierwohl und den intrinsischen Merkmalen Gesundheit und Geschmack (von Meyer-Höfer et al. 2015). Durch die Vermarktung von gesellschaftlich erwünschten Produktionsmethoden kann somit möglicherweise eine höhere Wertschöpfung und ein Vorteil für Unternehmen und Gesellschaft erzielt werden (Porter und Kramer 2011). Zusätzlich ist die Berücksichtigung gesellschaftlicher Ansprüche und Erwartungen für eine nachhaltige Nutztierhaltung von hoher Bedeutung, da Bürger in ihrer Rolle als Verbraucher, aber auch als Wähler langfristig mitentscheiden, was am Markt existieren kann (Allen et al. 1991; Buller und Roe 2012; Weary et al. 2016).

Weitere Studien sind jedoch notwendig, um die Kauf- und Zahlungsbereitschaft für Produkte aus den jeweiligen Haltungssystemen $\mathrm{zu}$ ermitteln und Marktpotenziale und Vermarktungsmöglichkeiten abschätzen zu können. Während die Weidehaltung für Milchkühe eher geringe Kostennachteile gegenüber der reinen Stallhaltung aufweist (Gillespie und Nehring 2014), stößt sie bei Wachstumsprozessen zurzeit technologisch an Grenzen. Innovative Haltungssysteme, die Weidegang für große Milchviehbetriebe ermöglichen würden, wären ein spannender Ansatzpunkt. Auch sollten betriebswirtschaftliche Berechnungen die Kostenunterschiede zwischen Weidesystemen tierartenspezifisch analysieren. Der vorliegende Beitrag kann eine solche Gesamtbewertung der Haltungssysteme als Voraussetzung einer umfassenden Diskussion noch nicht leisten, er zeigt aber durch den Verweis auf die hohen Akzeptanzprobleme der reinen Stallhaltung auf, dass eine umfassende Bewertung notwendig ist und z.B. der derzeitige Rückgang der Weidehaltung beim Milchvieh das gute Image der Milchwirtschaft bedroht (Reijs et al. 2013). Für Mastschweine könnten innovative Haltungssysteme mit Auslauf wie z. B. das Pig-Port-System interessante Perspektiven bieten. Für Masthühnchen sind Systeme mit Kaltscharrraum, wie sie z. B. Wiesenhof im Tierschutzlabelprogramm des Deutschen Tierschutzbund betreibt, eine produktionstechnisch gut funktionierende Haltungsform, die den Ablehnungsgrad von 83 auf $52 \%$ senkt.

\section{Literatur}

Albersmeier F, Spiller A (2010) Die Reputation der Fleischwirtschaft: eine Kausalanalyse. Ger J Agric Econ 59(4):258-270 
Allen P, Dusen D van, Lundy J, Gliessmann S (1991) Integrating social, environmental, and economic issues in sustainable agriculture. Am J Altern Agric 6:34-39

Boogaard BK, Oosting, SJ, Bock BB (2006) Elements of societal perception of farm animal welfare: a quantitative study in the Netherlands. Livest Sci 104:13-22

Buller H, Roe E (2012) Modifying and commodifying farm animal welfare: The economisation of layer chickens. J Rural Stud 33:141-149

Busch G, Schwetje C, Spiller A (2015) Bewertung der Tiergerechtheit in der intensiven Hähnchenmast durch Bürger anhand von Bildern: ein Survey Experiment. Ger J of Agric Econ 64(3):131-147

Cardoso CS, Hötzel MJ, Weary DM, Robbins JA, von Keyserligk MAG (2016) Imagining the ideal dairy farm. J Dairy Sci 99:1663-1671

Conner DS, Campbell-Arvai V, Hamm MW (2008) Consumer preferences for pasture-raised animal products: Results from Michigan. J of Food Distrib Res 39(2):12-25

Dawkins MS, Bonney R (2008) The future of animal farming - Renewing the ancient contract. Blackwell, London

Gillespie J, Nehring R (2014) Pasture-Based versus Conventional Milk Production: Where is the Profit? J of Agric and Appl Econ 46(4):543-558

Isermeyer F (2014) Künftige Anforderungen an die Landwirtschaft: Schlussfolgerungen für die Agrarpolitik. Thünen Working Paper 30:1-35

Kayser M, Schlieker K, Spiller A (2012) Die Wahrnehmung des Begriffs „Massentierhaltung“ aus Sicht der Gesellschaft. Ber über Landwirtsch 90(3):417-428

Kayser M, Spiller A (2012) Das Image der verschiedenen Fleischarten aus KonsumentInnen-Sicht. In:
Hambrusch J, Hoffmann C, Kantelhardt J, OedelWieser $\mathrm{T}$ (Hrsg.) Jahrbuch der österreichischen Gesellschaft für Agrarökonomie 21(1):23-31

Lassen J, Sandøe P, Forkman B (2006) Happy pigs are dirty! - conflicting perspectives on animal welfare. Livest Sci 103:221-230

Meyer-Höfer M v, Nitzko S, Spiller A (2015) Is there an expectation gap? Consumers' expectations towards organic. Br Food J 117(5):1527-1546

Porter ME, Kramer MR (2011) Creating shared value. Harv Bus Rev Jan./Febr:63-70

Reijs JW, Daatselaar CHG, Helming JFM, Jager J (2013) Grazing dairy cows in North-West Europe. Economic farm performance and future developments with emphasis on the Dutch situation. LEI Report 2013-001. The Hague, NL: LEI Wageningen UR

Sonntag W, Kaiser A, von Meyer-Höfer M, Spiller A (2017) Wie können Ansprüche der Gesellschaft in mögliche Veränderungsprozesse eingebunden werden? Konfrontation von Verbrauchern mit Zielkonflikten aus der Schweinehaltung. Ber über Landwirtsch 95(1):1-27

Vanhonacker F, Verbeke W (2014) Public and consumer policies for higher welfare food products: Challenges and opportunities. J Agric Environ Ethics 27:153-171

Weary DM, Ventura BA, von Keyserlingk MAG (2016) Societal views and animal welfare science: understanding why the modified cage may fail and other stories. Animal 10(2):309-317

Weinrich R, Kühl S, Zühlsdorf, A Spiller A (2014) Consumer Attitudes in Germany towards Different Dairy Housing Systems and their implications for the Marketing of Pasture Raised Milk. IFAMR 17(4):205-222 


\section{Analyse der Wahrnehmung der Nutztierhaltung durch unterschiedliche gesellschaftliche Gruppen}

Anja Rovers ${ }^{1}$, Christiane Wildraut ${ }^{2}$, Marcus Mergenthaler ${ }^{2}$, Winnie Isabel Sonntag ${ }^{3}$, Marie von MeyerHöfer $^{3}$, Achim Spiller ${ }^{3}$, Jörg Luy ${ }^{4}$, Doreen Saggau ${ }^{1}$, Nanke Brümmer ${ }^{1}$, Inken Christoph-Schulz ${ }^{1}$

${ }^{1}$ Thünen-Institut für Marktanalyse, Braunschweig

${ }^{2}$ Fachhochschule Südwestfalen, Fachbereich Agrarwirtschaft, Soest

${ }^{3}$ Georg-August-Universität Göttingen, Department für Agrarökonomie und Rurale Entwicklung, Marketing für Lebensmittel und Agrarprodukte

${ }^{4}$ Privates Forschungs- und Beratungsinstitut für angewandte Ethik und Tierschutz INSTET gGmbH, Berlin

anja-karolina.rovers@thuenen.de

\section{Einleitung}

In Deutschland zeigt sich bei der Nutztierhaltung eine wachsende Diskrepanz zwischen den derzeit vorherrschenden Haltungsverfahren und gesellschaftlichen Wünschen (Kayser et al. 2012). Tierwohl und unterschiedliche ethische Bewertungen der Nutztierhaltung sind im öffentlichen Diskurs präsent (WBA 2015). Untersuchungen deuten immer wieder darauf hin, dass eine gesellschaftliche Mehrheit ein eher negatives Bild hat, v.a. bei intensiven Haltungssystemen (bspw. Lemke et al. 2006; Weible et al. 2016). Werden die Haltungsbedingungen der Nutztierarten miteinander verglichen, wird vor allem die Haltung von Schweinen und Geflügel kritisiert und als verbesserungswürdig empfunden (Europäische Kommission 2007; Kayser et al. 2012). Im Gegensatz dazu wird die Milchviehhaltung deutlich positiver eingeschätzt (Europäische Kommission 2005; Christoph-Schulz et al. 2015). Der WBA hat vor diesem Hintergrund ein Gutachten mit neun Leitlinien für eine zukunftsfähige, in weiten Teilen der Bevölkerung akzeptierte Nutztierhaltung erstellt.

Im vorliegenden qualitativen Beitrag wird die Wahrnehmung der heutigen Nutztierhaltung basierend auf den Leitlinien des WBA-Gutachtens detaillierter untersucht. Für die Tierarten Rind, Schwein und Huhn wird die Wahrnehmung aus Sicht von Bürgern ${ }^{5}$ und Landwirten differenziert erfasst.

\footnotetext{
5 Der Begriff „Bürger“ wird hier in Abgrenzung zum Begriff „Landwirte“ verwendet und beinhaltet alle in Deutschland lebenden Personen ohne einen beruflichen Bezug zur Landwirtschaft oder Lebensmittelindustrie bzw. ohne entsprechende Ausbildungen/Studienabschlüsse.
}

\section{Methodische Vorgehensweise}

Die Wahrnehmung der Nutztierhaltung wurde mit Hilfe von leitfadengestützten Gruppendiskussionen explorativ erhoben. Bei Gruppendiskussionen gibt die Diskussionsleitung das Thema vor und lenkt den weiteren Verlauf der Gesprächsrunde, indem, gemäß Leitfaden, gezielt offene Fragen an die Gruppe gestellt werden. Vorteilhaft daran ist, dass viele Ergebnisse erst aufgrund der Diskussion zum jeweiligen Thema in einem dynamischen Prozess entstehen und auch unerwartete Aspekte auftreten (Wilson 1997; Halkier 2010), was mit standardisierten Befragungen nicht möglich ist. Die Ergebnisse sind ohne Anspruch auf Repräsentativität, liefern jedoch detaillierte Einblicke zur Frage, wie die Gesellschaft die Haltung der verschiedenen Tierarten einschätzt, was Hauptkritikpunkte sind und welche Aspekte eine eher untergeordnete Rolle spielen. Die Gruppendiskussionen fanden pro Tierart in je zwei (Landwirte) bzw. drei/vier (Bürger) Orten von September bis November 2015 statt. $^{6}$ Jede Diskussionsrunde hatte vier bis elf Teilnehmer und wurde in den folgenden Orten durchgeführt (Tab. 1):

Tab. 1 Orte der Gruppendiskussionen mit Landwirten und Bürgern

\begin{tabular}{|c|c|c|}
\hline Tierart & Landwirte & Bürger \\
\hline \multirow[t]{3}{*}{ Rind } & $\begin{array}{l}\text { Schleswig (Schleswig- } \\
\text { Holstein) }\end{array}$ & $\begin{array}{l}\text { Schwerin (Mecklenburg- } \\
\text { Vorpommern) }\end{array}$ \\
\hline & \multirow[t]{2}{*}{ Kempten (Bayern) } & Essen (Nordrhein-Westfalen) \\
\hline & & Kempten (Bayern) \\
\hline \multirow[t]{4}{*}{ Schwein } & \multirow{2}{*}{$\begin{array}{l}\text { Borken (Nordrhein- } \\
\text { Westfalen) }\end{array}$} & Oldenburg (Niedersachsen) \\
\hline & & Fulda (Hessen) \\
\hline & Thürkow & Halle (Sachsen-Anhalt) \\
\hline & $\begin{array}{l}\text { (Mecklenburg- } \\
\text { Vorpommern) }\end{array}$ & \\
\hline \multirow[t]{4}{*}{ Huhn } & \multirow{2}{*}{$\begin{array}{l}\text { Frisoythe } \\
\text { (Niedersachsen) }\end{array}$} & Hamburg (Hamburg) \\
\hline & & Vechta (Niedersachsen) \\
\hline & \multirow{2}{*}{$\begin{array}{l}\text { Magdeburg (Sachsen- } \\
\text { Anhalt) }\end{array}$} & Würzburg (Bayern) \\
\hline & & Erfurt (Thüringen) \\
\hline
\end{tabular}

Die Landwirte wurden mit Hilfe von Berufsverbänden zu den Diskussionsrunden eingeladen. Dabei waren pro Runde mindestens eine weibliche Betriebsleiterin sowie Vertreter unterschiedlicher Produktionsstufen der jeweiligen Tierart vertreten. Die Bürger wurden auf Basis bestimmter Quoten ${ }^{7}$

\footnotetext{
${ }^{6}$ Mit den Bürgern wurde jeweils in zwei Runden pro Ort diskutiert.

${ }^{7}$ Alter (20 bis 70 Jahre), Geschlecht (33\% bis $67 \%$ weiblich).
} 
durch ein Marktforschungsunternehmen akquiriert. ${ }^{8}$

Der Diskussionsleitfaden für die Landwirte enthielt offene Fragen zu den Gründen für die Wahl der jeweiligen Haltungsform, zu ihrer Wahrnehmung darüber, zur Wahrnehmung der gesellschaftlichen Debatte um die Nutztierhaltung, zu den betrieblichen Möglichkeiten der Tierhalter, sich für mehr Tierwohl zu engagieren sowie zu ihren Wünschen an Politik und Gesellschaft. Der Diskussionsleitfaden für die Bürger war so gestaltet, dass sich die offen gestellten Fragen an den Leitlinien aus dem WBAGutachten orientierten. Alle Diskussionen hatten eine Länge von bis zu 120 Minuten und wurden aufgezeichnet. Die anschließend erstellten Transkripte wurden einer qualitativen Inhaltsanalyse mithilfe von MAXQDA unterzogen und kategorisiert (vgl. Mayring 2002).

\section{Ergebnisse}

\subsection{Tierartenübergreifende Ergebnisse}

Folgende ausgewählte Ergebnisse sind für alle Diskussionsrunden mit den Landwirten festzuhalten: Im Hinblick auf Informationsmöglichkeiten über Landwirtschaft und Tierhaltung erläutern Landwirte eine Vielzahl von Optionen, wie bspw. einen „Tag der offenen Hoftür“. Gleichzeitig beklagen sie, dass die von ihnen und der Branche angebotenen Informationsmöglichkeiten nicht umfassender genutzt werden. Sie kritisieren zudem die einseitige und reißerische Berichterstattung der Medien. Landwirte verweisen auf wirtschaftliche Rahmenbedingungen und Zusatzkosten einer verbesserten Nutztierhaltung. Sie sehen trotz der geäußerten gesellschaftlichen Forderungen keine am Point-ofSale gezeigten Mehrzahlungsbereitschaften. Für die Landwirte ist das als Hinweis zu verstehen, dass Bürgern das Anliegen Tierwohl hauptsächlich dann wichtig ist, wenn sie keine Mehrkosten tragen müssen.

Der Einsatz von Technik in der Tierhaltung wird von den Landwirten als Anpassungsstrategie an wirtschaftliche Rahmenbedingungen gesehen. Der technische Fortschritt bedeute Erleichterung der eigenen körperlichen Arbeit und habe positive Effekte für das Tierwohl, weil durch Technik eine bessere Tierversorgung und -überwachung

\footnotetext{
8 Der Meinungsaustausch innerhalb der Diskussionen zwischen Personen mit unterschiedlicher Ernährungsweise war ausdrücklich erwünscht. Daher waren neben Personen, die Fleisch essen, auch max. zwei Personen pro Gruppe vertreten, die sich vegetarisch oder vegan ernähren.
}

ermöglicht werde bzw. gewonnene zeitliche Freiräume für eine intensivierte Tierbetreuung genutzt werden könnten. Der Kritik am Medikamenteneinsatz halten Landwirte entgegen, dass für sie eine Gefährdung der Tiergesundheit durch einen ausbleibenden Medikamenteneinsatz nicht mit Tierschutz und Tierwohl zu vereinbaren ist. Einschränkungen beim Medikamenteneinsatz lehnen Landwirte deshalb ab. Die Landwirte sehen ihre Nutztiere als Produktionsgrundlage und grenzen die Nutztierhaltung deutlich von der Heimtierhaltung ab. Bei den Bürgern kritisieren sie Anthropomorphisierungstendenzen gegenüber Tieren.

Nachfolgend werden ausgewählte Ergebnisse der Gruppendiskussionen mit den Bürgern vorgestellt: Die Diskussionsteilnehmer betonen, dass es heutzutage immer weniger landwirtschaftliche Betriebe gäbe. Dadurch sei es schwerer geworden, persönlichen Kontakt zur Landwirtschaft zu haben. Deshalb seien die Medien oftmals die einzige Informationsquelle. Die mediale Berichterstattung sei allerdings meist negativ und zeige oft „Horrorbilder“. Man müsse daher kritisch reflektieren, woher die Informationen stammen und versuchen, starke Beeinflussung zu vermeiden. Direktvermarktung sei eine gute Möglichkeit, Einblicke in die Land wirtschaft zu erhalten.

Häufig verwenden die Teilnehmer den Begriff „Massen(tier)haltung“. Dieser wird meist mit fehlendem Freilandzugang, zu geringem Platzangebot und intransparenten, abgeschlossenen Systemen verbunden, v.a. beim Schwein und beim Huhn. Ausnahmen seien Biobetriebe. Die Diskutanten heben häufig hervor, dass heutzutage viel Technik eingesetzt würde, um verschiedene Prozesse, wie z.B. die Fütterung, automatisiert durchzuführen. Die Teilnehmer vermuten dadurch einen verminderten Mensch-Tier-Kontakt.

Beim Thema Medikamenteneinsatz ist am häufigsten von Antibiotika die Rede. Dabei wird erwähnt, dass es Rückstände in Fleisch oder Eiern gäbe, mit denen die Gefahr von antibiotikaresistenten Bakterien einhergehe. Teilweise wird hierbei betont, dass Antibiotika prophylaktisch (z.B. mit dem Futter) gegeben würden. Beim Schwein ist außerdem häufig von leistungssteigernden Medikamenten oder von Hormonen die Rede. Teilweise werden auch Rückstände von Medikamenten in der Milch vermutet. Oft wird angenommen, dass der Medikamenteneinsatz infolge der als schlecht und nicht artgerecht eingeschätzten Haltungsbedingungen erforderlich sei und verringert werden könnte, wenn sich die Haltung verbessern würde. 
Einige Diskutanten betonen, dass Tiere generell fühlende Lebewesen seien. Besonders beim Schwein werden einzelne Haltungsbedingungen, v.a. das geringe Platzangebot oder die Lichtverhältnisse im Stall, als abträglich für das Wohlbefinden der Tiere eingestuft. Andere Teilnehmer bekräftigen, dass es einem Tier generell nur dann gut gehen würde, wenn es seine natürlichen Bedürfnisse erfüllen könne.

\subsection{Tierartenspezifische Ergebnisse}

Aus den Diskussionen mit den Landwirten lassen sich folgende, ausgewählte Ergebnisse für die drei Tierarten festhalten:

\section{Rind:}

Milchviehhalter betrachten die Fütterung von Milchkühen als wichtige Managementaufgabe. In ihren Augen erfordern die erreichten züchterischen Entwicklungen zu hohen Milchleistungen eine bedarfsgerechte Fütterung der einzelnen Tiere, die ohne Kraftfuttergaben nicht erreicht werden könne. Sie sehen weiteren Entwicklungen der Automatisierung und Technisierung in den Ställen mit großem Interesse entgegen. Als positiv bewerten sie u.a. die dadurch entstehenden Freiräume für die Tierhalter und den Komfort für die Tiere, beispielsweise wenn Milchkühe individuelle Melkzeiten eines Melkroboters nutzen können. Die Weidehaltung sehen Landwirte kontrovers. Insbesondere in Betrieben mit größeren Tierbeständen und knappen arrondierten Flächen stellen in ihren Augen moderne Laufställe mit Bewegungsfreiheit für die Tiere und verschiedenen Funktionsbereichen einen tierwohlgerechten Kompromiss dar.

\section{Schwein:}

Eingriffe am Tier, wie Kastration von männlichen Ferkeln, Zähneschleifen von Saugferkeln sowie Kupieren der Schwänze werden als Notwendigkeit zur Sicherstellung von Tierwohl in den derzeitigen Haltungssystemen betrachtet. Nicht thematisiert wird von Landwirten, dass diese Eingriffe derzeit gesetzlich teilweise nur als Ausnahme erlaubt sind. Landwirte sehen Kastenstände für Sauen der Gruppenhaltung als überlegen an, weil damit die tierindividuelle Beobachtung vereinfacht wird und rangniedere Sauen besser geschützt werden. Auslauf und Beschäftigung werden von Landwirten sehr differenziert betrachtet. Beschäftigungsmöglichkeiten innerhalb der bestehenden Haltungssysteme sind für Landwirte einfach umzusetzen. Unter ästhetischen Gesichtspunkten wird Strohhaltung als interessante
Alternative gesehen. Auslaufmöglichkeiten werden aus finanziellen, tier- und seuchenhygienischen sowie behördlichen Gründen kritisch gesehen. Auch werden begrenzte Absatzpotenziale als Hemmnis angeführt.

\section{Huhn:}

Die Landwirte beklagen Unkenntnis der Bevölkerung zur Legehennenhaltung. Das Verbot der Käfighaltung von Legehennen sei bei vielen Verbrauchern nicht angekommen. Daraus schließen sie, dass Veränderungen nach den Wünschen der Gesellschaft letztlich zu keiner allgemein besseren Beurteilung der Tierhaltung führen. Die Legehennenhaltung wird von Landwirten als ein Bereich der Nutztierhaltung angeführt, in dem den Tieren heute mehr Platz und Bewegungsmöglichkeiten geboten werden. Dabei differenzieren sie die Effekte und weisen beispielsweise auf erhebliche erhöhte gesundheitliche und hygienische Probleme in der Freilandhaltung hin. Gleichzeitig gewinnen sie dieser Haltungsform durchaus auch ästhetische und emotionale Mehrwerte ab. In der intensiven Hühnchenmast sehen Landwirte eine hocheffiziente Fleischproduktion, die es Bürgern ermöglicht, günstig gesundes Fleisch einzukaufen, wofür der steigende ProKopf-Verbrauch von Geflügelfleisch spreche.

Die Inhaltsanalyse der Gruppendiskussionen mit den Bürgern liefert die folgenden, für die drei untersuchten Tierarten ausgewählten Ergebnisse:

Rind:

Die Bürger beschreiben Kühe auf der Weide als ihre Idealvorstellung, weil dies einer natürlichen Haltung entspreche. Einige Teilnehmer fragen sich jedoch, inwiefern die Tiere, wenn sie es nicht anders gewohnt seien, überhaupt die Weide vermissen würden. Einige Bürger räumen ein, dass Weidehaltung nicht immer umzusetzen sei und Milchkühe daher häufig auch nur im Stall gehalten würden. Gras und Heu seien das natürliche Futter für das Rind. Viele Teilnehmer räumen aber Informationsdefizite bezüglich der Fütterung ein und wüssten v.a. nicht, was im Kraftfutter enthalten sei. Im Hinblick auf Melkroboter ist das Bild heterogen. Während einige Teilnehmer diese favorisieren und sogar als „kuhfreundlich“ bezeichnen, berichteten andere, dass sie sie als äußerst negativ empfinden.

\section{Schwein:}

Aussagen zum Platzangebot für Schweine erfolgen sehr häufig und vordergründig. Es wird erwähnt, dass es „eng“ im Stall sei, die Tiere „eingepfercht“ 
gehalten würden oder dass „,kein Platz“ für die Tiere vorhanden sei. Besonders häufig werden negativ behaftete Adjektive in Verbindung mit Muttersauen in Kastenständen genannt, wobei der Begriff Kastenstand nicht explizit verwendet wird. Auf dessen Funktion wird durch die Teilnehmer nicht eingegangen. Wenn es um Einrichtungsgegenstände oder Beschäftigungsmöglichkeit geht, ist in einigen Aussagen von Bällen oder Ketten die Rede. Einige Diskutanten heben hervor, dass Schweine intelligente Tiere seien, die sich eigentlich Beschäftigung wünschen. Teilweise waren in den Diskussionen bis auf Impfungen keine weiteren Eingriffe am Schwein in der Vorstellung der Teilnehmer vorhanden. Daher wurde fallbedingt in die Runden gegeben, dass Ferkelkastration, Schwänze kupieren und Zähne abschleifen übliche Eingriffe seien. Am häufigsten nahmen die Diskutanten dann zur Kastration Stellung.

Huhn:

Das gesetzliche Verbot der konventionellen Käfighaltung ist bei Bürgern kaum präsent. Wenn in den Gruppen die Haltungsbedingungen von Legehennen und Masthühnchen thematisiert werden, beschreiben die Teilnehmer jeweils gitterartige Käfigsysteme. Insgesamt dominieren bei der Beschreibung der Wahrnehmung negative Begriffe, die zeigen, welch schlechtes Image die Geflügelhaltung bei deutschen Bürgern hat. Positive Assoziationen wurden mit Freilandhaltung und ökologischer Haltung geäußert. Ferner wird ein hoher Medikamenten- und Antibiotikaeinsatz vermutet. Ein Großteil der Teilnehmer war davon überzeugt, dass die Tiere präventiv mit Antibiotika behandelt werden. Es wurde in diesem Zusammenhang die Sorge vor Resistenzbildung, antibiotikahaltigen Produkten (Eier und Hühnchenfleisch) und eines damit einher gehenden, negativen Einflusses auf die eigene Gesundheit geäußert.

\section{Zusammenfassung, Diskussion und Ausblick}

$\mathrm{Zu}$ allen Diskussionen können jeweils für Landwirte und Bürger folgende tierartenübergreifende, ausgewählte Ergebnisse aufgezeigt werden:

\section{Landwirte}

- Die Gesellschaft gewinnt Informationen über die Landwirtschaft überwiegend aus den Medien.

- Es ist häufig unklar, wer die entstehenden Mehrkosten für verbesserte Nutztierhaltung tragen soll.

- Technik und automatisierte Prozesse erleichtern die Arbeit für Tierhalter und ermöglichen eine verbesserte Tierbeobachtung und -versorgung.
- Kranke Nutztiere sollen behandelt werden.

- Nutztiere dienen dem Menschen zur Lebensmittelproduktion.

Bürger

- Der direkte Bezug zu Landwirtschaft und Nutztierhaltung geht zurück.

- Die Haltungsbedingungen für Nutztiere sind vielfach verbesserungswürdig.

- Heutige Tierställe sind sehr technisiert und automatisiert, was den Mensch-Tier-Kontakt mindert.

- Der Einsatz von Medikamenten in der Nutztierhaltung wird sehr kritisch gesehen.

- Nutztiere haben Anspruch auf Fürsorge und Verantwortung durch den Menschen.

Die Analyse zeigt außerdem, dass es nicht um die Nutztierhaltung per se geht. So treten tierartenspezifische Unterschiede auf, die wiederum differenziert zwischen Landwirten und Bürgern festzustellen waren:

Rind

Landwirte

- Aufgrund der zu erbringenden Leistung müssen in der Milchviehfütterung auch Kraftfutter und andere Zusätze eingesetzt werden.

- Das Melken wird mit zunehmendem Automatisierungsgrad durchgeführt. Dies spart Zeit und Arbeit.

- In der Milchviehhaltung ist Weidegang nicht überall durchführbar.

Bürger

- Die alleinige Fütterung von Rindern mit Gras und Heu ist grundsätzlich erwünscht.

- Das Melken ist mit Hilfe von Maschinen und Computern ein technisierter Vorgang, was den direkten Kontakt zu den Milchkühen vermindern kann.

- In der Milchviehhaltung werden Weidegang und Bewegungsfreiheit als positiv wahrgenommen.

Schwein

Landwirte

- Die vorherrschenden technischen und wirtschaftlichen Rahmenbedingungen erlauben es nicht, auf bestimmte Eingriffe am Schwein zu verzichten.

- Die Haltung von Muttersauen in Kastenständen schützt die Ferkel.

- Auslauf und Beschäftigungsmöglichkeiten für Schweine machen die Produktion aufwendiger und teurer. 
Bürger

- Bestimmte Eingriffe am Schwein sind teilweise unerwünscht.

- Die Haltung von Muttersauen in Kastenständen wird als „einpferchen“ empfunden.

- Schweine sind intelligente Tiere, die Auslauf und Beschäftigungsmöglichkeiten brauchen.

Huhn

Landwirte

- Die Abschaffung der Käfighaltung ist bei vielen Bürgern nicht angekommen.

- Es gibt deutliche Verbesserungen in der Hühnerhaltung, wie bspw. mehr Platz oder Bewegungsmöglichkeiten.

- Durch eine kurze und intensive Mast kann dem Wunsch der Bürger entsprochen und Hühnchenfleisch günstig produziert werden.

Bürger

- Die Abschaffung der Käfighaltung ist vielen Bürgern nicht bekannt.

- Hühnerhaltung wird bis auf Auslaufsysteme eher negativ wahrgenommen.

- Es wird vermutet, dass in der Hühnerhaltung ein hoher Antibiotikaeinsatz stattfindet, der sich auf die Produktqualität und die eigene Gesundheit negativ auswirkt.

Wie frühere Studien zeigen, besitzen Landwirte und Bürger ein unterschiedliches Verständnis von Tierwohl (Vanhonacker et al. 2008). Entsprechend verdeutlichen die Ergebnisse auch eine teils unterschiedliche Wahrnehmung, z.B. wenn es um Kastenstände für Sauen geht oder um den Einsatz von Melkrobotern in der Milchviehhaltung. Die Ergebnisse decken sich mit bisher vorliegenden, teils ebenfalls qualitativen Studien. Beispielsweise wurde zum Thema Schwein erarbeitet, dass das Platzangebot von zentraler Bedeutung ist (bspw. Kayser et al. 2012; Wildraut et al. 2015; Weible et al. 2016). Die Diskussionsteilnehmer kritisieren außerdem fehlende Beschäftigungsmöglichkeiten für die schweine. Busch et al. (2015) konnten jedoch zeigen, dass diese - wenn im Stall vorhanden - selbst bei Vorlage von Bildmaterial von den Befragten in deren Studie mehrheitlich nicht als solche erkannt wurden. Dass die beim Bürger existierende Vorstellung von tatsächlich erlebten Eindrücken bei realen Stallbesichtigungen abweicht, haben Ermann et al. (2016) nachgewiesen. Sie schlussfolgern, dass Stallbesichtigungen insbesondere für Kritiker und
Medienvertreter ermöglicht werden sollten, um Transparenz zu gewährleisten. Auch die Teilnehmer der vorliegenden Erhebung - sowohl Landwirte als auch Bürger - berichten positiv von Direktvermarktung oder Aktionen wie einem Tag der offenen Hoftür. Beim Huhn sind dagegen bereits umgesetzte Maßnahmen, wie die Abschaffung der Käfighaltung in Deutschland, noch nicht grundsätzlich bei Bürgern bekannt.

Daraus lässt sich ableiten, dass Verbesserungen in der Tierhaltung schwierig für Bürger zu kommunizieren sind, um eine positive Einstellung gegenüber bereits umgesetzten Maßnahmen zu erzielen. Eine wirksame Kommunikation wird von Busch et al. (2015) als entscheidend für eine verbesserte gesellschaftliche Akzeptanz der Tierhaltung, die aus der Wahrnehmung generiert wird, erachtet. Mithilfe der erzielten Ergebnisse können noch keine Rückschlüsse auf die Wahrnehmung der Gesamtbevölkerung getroffen werden. Die vorliegenden Ergebnisse bedürfen daher weiterer Validierung in größeren Stichproben und bilden die Basis für standardisierte Befragungen mit Landwirten und Bürgern. Eine weiterführende Untersuchung des Dialogprozesses zwischen Landwirten und Bürgern nehmen Wildraut et al. (in diesem Beitrag) vor, der an wesentliche Erkenntnisse dieser Analyse anschließt und diese vertieft. Derzeit ist nicht abschätzbar, wie sich die Wahrnehmung der Nutztierhaltung im Zeitablauf konkret verändert hat. Um zu überprüfen, inwiefern Maßnahmen zur Verbesserung einen positiven Einfluss auf die Wahrnehmung haben, ist es unabdingbar, in regelmäßigen Abständen identische Fragen an die Bevölkerung zu stellen. Dabei dienen die bevorstehenden, quantitativen Befragungen als erste Befragungswelle.

Zudem besteht wenig Wissen darüber, wie Ställe für die verschiedenen Nutztierarten gestaltet sein müssten, die von den unterschiedlichen gesellschaftlichen Gruppen mehrheitlich (besser) akzeptiert werden würden. Vor diesem Hintergrund ist es erforderlich, verschiedene Prototypen $\mathrm{zu}$ entwickeln. $\mathrm{Zu}$ untersuchen wäre dann, wie Landwirte sie bezogen auf ihren Arbeitsalltag bewerten und wie Nutztierwissenschaftler und Bürger diese Stallneubauten beurteilen. Auf Basis dieser Ergebnisse wäre es weiter erforderlich, dass ggf. mehrere, nacheinander geschaltete Bewertungsrunden stattfinden. Ein derartiges Vorgehen entspricht dem Innovationscharakter des SocialLab, das sich u.a. zum Ziel gesetzt hat, neue Stallsysteme oder auch neue Züchtungsrassen aus der Perspektive unterschiedlicher gesellschaftlicher Gruppen zu untersuchen. 


\section{Literatur}

Busch G, Gauly S, Spiller A (2015) Wie wirken Bilder aus der modernen Tierhaltung der Landwirtschaft auf Verbraucher? Neue Ansätze aus dem Bereich des Neuromarketings. In: Schriftenreihe der Rentenbank, Band 31, Die Landwirtschaft im Spiegel von Verbrauchern und Gesellschaft, S. 67-94

Christoph-Schulz I, Salamon P, Weible D (2015) What is the benefit of organically reared dairy cattle? Societal perception towards conventional and organic dairy farming. Int J Food Syst Dyn 6(3):139-146

Ermann M, Graskemper V, Spiller, A (2016) Die Wirkung von geführten Stallbesichtigungen auf Bürger - eine Fallstudie auf nordwestdeutschen Schweinemastbetrieben. In: Schriften der Gesellschaft für Wirtschafts- und Sozialwissenschaften des Landbaues e.V., Band Nr. 52: Agrar- und Ernährungswirtschaft: Regional vernetzt und global erfolgreich (im Druck)

Europäische Kommission (2005) Attitudes of consumers towards the welfare of farmed animals, Eurobarometer Spezial 229. Verfügbar unter: http://ec.europa.eu/food/animal/welfare/euro_baro meter25_en.pdf

Europäische Kommission (2007) Special Eurobarometer 229 (2): Attitudes of consumers towards the welfare of farmed animals, wave 2. Brussels. Verfügbar unter: http://ec.europa.eu/food/animal/ welfare/survey/sp_barometer_fa_en.pdf

Halkier B (2010) Focus groups as social enactments: integrating interaction and content in the analysis of focus groups data. Qual Res 10(1):71-89

Kayser M, Schlieker K, Spiller A. (2012) Die Wahrnehmung des Begriffs „Massentierhaltung“ aus Sicht der Gesellschaft. In: Berichte über Landwirtschaft, Heft 90, Nummer 3:417-428
Lemke D, Schulze B, Spiller A, Wocken C (2006) Verbrauchereinstellungen zur modernen Schweinehaltung: Zwischen Wunsch und Wirklichkeit. Wien: Jahrbuch der Österreichischen Gesellschaft für Agrarökonomie

Mayring P (2002) Einführung in die Qualitative Sozialforschung. Eine Anleitung $\mathrm{zu}$ qualitativem Denken. Beltz Verlag, Weinheim und Basel

Vanhonacker F Verbeke W van Poucke E, Tuyttens F (2008) Do citizens and farmers interpret the concept of farm animal welfare differently? Livest Sci 116:126-136

Weible D, Christoph-Schulz I, Salamon P, Zander K (2016) Citizens' perception of modern pig production in Germany: a mixed-method research approach. Brit Food J 118(8):2014-2032

Wildraut C, Plesch G, Härlen I, Simons J, Hartmann M, Ziron M, Mergenthaler, M (2015) Multimethodische Bewertung von Schweinehaltungsverfahren durch Verbraucher anhand von Videos aus realen Schweineställen. Forschungsberichte des Fachbereichs Agrarwirtschaft Soest, Nr. 36

Wilson V (1997) Focus groups: a useful qualitative method for educational research. BERJ 23(2):209-224

WBA Wissenschaftlicher Beirat Agrarpolitik beim BMEL (2015) Wege zu einer gesellschaftlich akzeptierten Nutztierhaltung. Gutachten. Berlin

Zander K, Isermeyer F, Bürgelt D, Christoph-Schulz I, Salamon P, Weible D (2013) Erwartungen der Gesellschaft an die Landwirtschaft. Münster: Stiftung Westfälische Landwirtschaft 
Annäherungen in der Bewertung der landwirtschaftlichen Nutztierhaltung - Ergebnisse aus gemeinsamen Diskussionsrunden mit Tierhaltern und Verbrauchern

Christiane Wildraut ${ }^{1}$, Anja Rovers ${ }^{2}$, Winnie Sonntag ${ }^{3}$, Inken Christoph-Schulz ${ }^{2}$, Marie von Meyer-Höfer ${ }^{3}$, Jörg Luy ${ }^{4}$, Jenny Wolfram ${ }^{1}$, Achim Spiller ${ }^{3}$, Marcus Mergenthaler $^{1}$

${ }^{1}$ Fachhochschule Südwestfalen, Fachbereich Agrarwirtschaft, Soest

${ }^{2}$ Thünen-Institut für Marktanalyse, Braunschweig

${ }^{3}$ Georg-August-Universität Göttingen, Department für Agrarökonomie und Rurale Entwicklung, Marketing für Lebensmittel und Agrarprodukte

${ }^{4}$ Privates Forschungs- und Beratungsinstitut für angewandte Ethik und Tierschutz INSTET gGmbH, Berlin

wildraut.christiane@fh-swf.de

\section{Einleitung}

Das Verhältnis zwischen Landwirtschaft und Gesellschaft hat sich in den vergangenen Jahren zunehmend distanziert (Wildraut et al. 2015). Obwohl eine insgesamt positive Grundhaltung seitens der Gesellschaft gegenüber der Landwirtschaft dokumentiert ist (Zander et al. 2013; Helmle 2011), wird die Branche in der gesellschaftlichen Diskussion häufig und zunehmend scharf kritisiert. Die Kritik an der landwirtschaftlichen Nutztierhaltung hängt maßgeblich mit einem wahrgenommenen Mangel an Tierwohl und unzureichender Berücksichtigung der Bedürfnisse der Tiere zusammen (Vanhonacker et al. 2012; WBA 2015). Entwicklungen zu größeren Tierbeständen in der modernen Nutztierhaltung und eine damit einhergehende fortschreitende Technisierung der Verfahren mit wenig Einblick in Tierställe und einer geringen Transparenz der Tierhaltung insgesamt fördern das Unbehagen bei Verbrauchern (Busch et al. 2013). Insgesamt sind sich Verbraucher jedoch unsicher, wann es den Tieren eher schlecht oder eher gut geht. Ihre Kriterien zur Bewertung von Tierhaltungsverfahren und Tierwohl sind störanfällig (Wildraut et al. 2015).

Landwirte sehen derzeitige Tierhaltungssysteme als positiv und innovativ, begründen ihre Einschätzungen zum Tierwohl über Gesundheits- und Leistungsparameter und bewerten aktuelle Verfahren der Tierhaltung als förderlich für das Tierwohl (Te Velde et al. 2002; Vanhonacker et al. 2008). Sie beklagen die oftmals idealisierte „Museumslandwirtschaft“ der
Verbraucher (vgl. Simons et al. in diesem Heft), die sie als entfremdet wahrnehmen und erachten die Forderungen nach einem verbesserten Tierschutz in der intensiven Tierhaltung als schwer umsetzbar.

So besteht zwischen der Wahrnehmung von Tierhaltungsverfahren durch Verbraucher und durch Landwirte eine deutliche Diskrepanz (Vanhonacker et al. 2008). Die Landwirtschaft insgesamt und auch einzelne Personen bzw. die Landwirtsfamilien sehen sich mittlerweile zunehmend unter Rechtfertigungsdruck und bemängeln ihrerseits eine Kluft zwischen stetig steigenden moralischen Ansprüchen der Gesellschaft an die landwirtschaftliche Erzeugung auf der einen und unverändert niedrigen Zahlungsbereitschaften auf der anderen Seite (Vierboom et al. 2015). Der fehlende Konsens zwischen Landwirten und Verbrauchern in Bezug auf die Nutztierhaltung führt bei den Tierhaltern zu Angst vor einem Verlust der sozialen Akzeptanz und der "Licence to produce" (Busch et al. 2013; Te Velde et al. 2002).

In die brancheninterne Diskussion zur Weiterentwicklung von Tierhaltungsverfahren und die Verbesserung des Tierwohls werden Verbraucher bislang nicht strukturiert einbezogen. Dabei scheint es problematisch, wenn sich die Nutztierhaltung wenig am öffentlichen Diskurs beteiligt und stattdessen mit Rückzug oder Abschottung reagiert (WBA 2015). Bisherige Kommunikationsstrategien der Branche und auch einzelner Landwirte für mehr Konsens mit der Gesellschaft im Hinblick auf die aktuellen Tierhaltungsverfahren beschränken sich häufig auf einseitig ausgerichtete Aufklärungskonzepte. Vor dem Hintergrund der anhaltenden Diskussionen scheinen sie nicht auszureichen, um die Kluft zwischen Verbraucher und Landwirten bei der Bewertung der Verfahren und des Tierwohls zu verringern und die Konflikte zu Fragen aktueller und künftiger Tierhaltungsverfahren zu lösen. Um eine langfristige Akzeptanz- und Imageverbesserung $\mathrm{zu}$ erreichen, werden weitere Kommunikationsansätze und -instrumente mit Prozessen gegenseitigen Lernens empfohlen (Spiller et al. 2016). Gleichzeitig fehlen allerdings Untersuchungen zum Einsatz und zur Wirksamkeit neuer Instrumente.

Um diesem Defizit abzuhelfen, zielt die vorliegende Teilstudie darauf $\mathrm{ab}$, die Einstellungen zur Nutztierhaltung und zum Tierwohl vor, während und nach gemeinsamen Gruppendiskussionen mit Verbrauchern und Tierhaltern zu analysieren. Damit soll ein Beitrag für ein besseres Verständnis des Konflikts und der Kommunikation zwischen Landwirten und 
Verbrauchern in Bezug auf moderne Verfahren der Tierhaltung geleistet werden. Darüber hinaus soll untersucht werden, inwieweit persönliche Begegnungen in Form von moderierten Gruppendiskussionen dazu geeignet sind, die Kluft zwischen Landwirtschaft und Gesellschaft zu verringern.

\section{Daten und Methode}

Im Rahmen eines gemischt-methodischen Untersuchungsdesigns wurden im März und April 2016 in 6 unterschiedlichen Schwerpunktregionen der Tierhaltung in Deutschland gemeinsame Gruppendiskussionen mit insgesamt 26 Verbrauchern und 26 Landwirten (je Erhebung 4 bis 6 Verbraucher und eine äquivalente Anzahl Landwirte) zu aktuellen Tierhaltungsverfahren durchgeführt. Jeweils 2 Diskussionsrunden bezogen sich auf die Tierarten Schwein (Nordrhein-Westfalen und Mecklenburg-Vorpommern), Rind (Schleswig-Holstein und Bayern) und Geflügel (Niedersachsen und SachsenAnhalt). Die Auswahl der Befragungsregionen erfolgte mit dem Ziel der Berücksichtigung von Viehdichten für die 3 Tierarten in Nord-, Süd-, Ostund Westdeutschland (bezogen auf Statistische Ämter des Bundes und der Länder 2011).

Die Auswahl und Rekrutierung der Landwirte erfolgte über die berufsständische Vertretung auf der jeweiligen Landesebene. Die Verbraucher wurden über ein Marktforschungsinstitut rekrutiert. Quotierungsvorgabe für die Auswahl der Landwirte war eine Einbeziehung verschiedener Produktionssysteme und die Teilnahme von mindestens einer Frau. Die Verbraucherstichprobe wurde nach Alter (18 bis 70 Jahre), Geschlecht (mindestens $50 \%$ weiblich) und Erwerbstätigkeit (mindestens $33 \%$ erwerbstätig) quotiert, um eine breite Datengrundlage zu erreichen. Der Konsum von tierischen Produkten (in Bezug auf die 3 Tierarten als Diskussionsthema) war Voraussetzung für die Teilnahme der Verbraucher an der Untersuchung. Personen mit landwirtschaftlichem Hintergrund waren von der Verbraucherstichprobe ausgeschlossen.

Als Leitfaden für die moderierten Diskussionen mit den Teilnehmern dienten Thesen zur Tierhaltung, die im Rahmen von zuvor getrennt durchgeführten Gruppendiskussionen mit Landwirten und Bürgern als konfliktbehaftet abgeleitet worden waren (vgl. Rovers et al. in diesem Beitrag) und seitens der Moderation in die Gruppe gegeben wurden. Für jede
These stand eine Diskussionszeit von 10 Minuten zur Verfügung.

Um zu erfassen, ob sich die Meinungen der Landwirte und der Verbraucher während ihrer Diskussion über die benannten Konflikte der Tierhaltung ändern, wurde das qualitative Vorgehen in den Gruppendiskussionen um einen quantitativen Part ergänzt (Tashakkori \& Teddlie 1998). Dazu wurde vor und nach den Gruppendiskussionen jeweils die Zustimmung zu Statements mit inhaltlichem Bezug zu den diskutierten Thesen anonym und standardisiert anhand einer Skala abgefragt (Tab. 1). Völlige Ablehnung der Statements entsprach dem Wert 1 auf der Skala, völlige Zustimmung entsprach dem Wert 9. Zusätzlich wurde die Option „Kann ich nicht beurteilen" angeboten.

Tab. 1 Eingesetzte Statements vor und nach gemeinsamen Gruppendiskussionen mit Tierhaltern und Verbrauchern

Allgemeine Statements

1A Unsere Gesellschaft hat immer weniger direkten Bezug zur Landwirtschaft

1B Zur Landwirtschaft kann man sich nur über die Medien informieren

2A Nutztiere sollten bessere Haltungsbedingungen bekommen

2B Niemand will die entstehenden Mehrkosten für bessere Haltungsbedingungen von Nutztieren tragen

3A Heutige Tierställe sind sehr technisiert und automatisiert

3B Die Arbeit für Tierhalter wird durch Technik leichter und die Nutztiere können besser versorgt werden

4A Der Einsatz von Medikamenten in der Tierhaltung ist kritisch zu sehen

4B Nutztiere sollen behandelt werden, wenn sie krank sind

$5 \mathrm{~A}$ Nutztiere haben Anspruch auf Fürsorge und Verantwortung durch den Menschen

5B Nutztiere sollen uns in erster Linie zur Lebensmittelproduktion dienen

In der Auswertung wurde die Gruppe der Tierhalter der Gruppe der Verbraucher gegenübergestellt, um zu prüfen, ob die inhaltliche Auseinandersetzung mit der Thematik eine Veränderung der Beurteilung der Statements bei Landwirten und Verbrauchern auslöst. Dazu wurden zunächst die Mittelwerte der Bewertungen der Statements durch Landwirte einerseits und Verbraucher andererseits berechnet und die Differenzen auf signifikante Unterschiede durch den nichtparametrischen Mann-Whitney-U-Test getestet. Im Anschluss wurden die Mittelwerte vor den Diskussionen den Werten nach den Diskussionen im nicht 
parametrischen Wilcoxon-Vorzeichen-Rang-Test gegenübergestellt und auf signifikante Unterschiede getestet. Die beiden genannten Tests liefern auch in kleineren Stichproben robuste Ergebnisse.

Die moderierten Gruppendiskussionen wurden als Audioaufnahme aufgezeichnet. Anschließend erfolgten eine Transkription und eine qualitative Inhaltsanalyse (basierend auf Mayring 2002). Hierbei wurden über ein Kategoriensystem mit Codes und Subcodes Textstellen markiert und die Texte damit für die weitere dokumentenübergreifende Analyse strukturiert, z.B. bezogen auf inhaltliche Aspekte oder auf Kommunikationsmuster der Teilnehmer.

\section{Ergebnisse}

Die diskutierten Thesen, die sowohl für Verbraucher als auch für Landwirte Dilemmata darstellen, werden von beiden Gruppen teilweise ähnlich bewertet, teilweise aber auch kontrovers gesehen. Deutlich wird das am Grad der mittleren Zustimmung zu den abgefragten Statements vor den Diskussionen, der die Ausgangsstandpunkte beider Gruppen widerspiegelt, mit denen sie in den Dialog eintreten. (Tab. 2). Die größte Zustimmung sowohl seitens der Verbraucher als auch seitens der Tierhalter erfahren vor den Diskussionen die Statements 5A: „Nutztiere der Diskussionen und nach den Diskussionen keine Änderungen in den Bewertungen erfahren. Darin zeigt sich verbraucherseitig die hohe Bedeutung des Tierschutzgedankens, der auch ethisch begründet wird, wie dieses Zitat zeigt: „Ich finde, man sollte ein Tier trotzdem voller Respekt behandeln, auch wenn es keinen Namen trägt ${ }^{\prime} .{ }^{9}$ Die Tierhalter brachten ein besonderes Verhältnis zu ihren Tieren zum Ausdruck, welches sich vom Verhältnis der Industrie bzw. des Gewerbes zu den dortigen „Produktionsfaktoren“ grundsätzlich unterscheidet. Sie betonen sowohl ein professionelles als auch ein dazugehöriges emotionales Verhältnis zu ihren Tieren: „Es ist ja unsere Grundlage. Die Grundlage unseres Wirtschaftens sind die Tiere und der Boden. Und damit gehen wir so gut um, wie es nur geht“. Die moralische Verpflichtung im Umgang mit kranken Tieren sehen die Landwirte als berufliche Selbstverständlichkeit und alternativlos: „Wenn Tiere krank sind, müssen sie behandelt werden. Da gibt es gar keine Diskussion, das ist Tierschutz und Tierwohl“. Die Verbraucher teilen diese Einschätzung, vermuten jedoch, dass der Medikamenteneinsatz durch Änderungen der Haltungsbedingungen reduziert werden könnte: „Viele Erkrankungen entstehen ja dadurch, dass die Tiere so eng gehalten werden“.

Tab. 2 Bewertung von Statements vor und nach gemeinsamen Gruppendiskussionen mit Tierhaltern und Verbrauchern

\begin{tabular}{|c|c|c|c|c|c|c|c|c|c|c|c|c|c|}
\hline \multirow[t]{2}{*}{ Art der Bewertungsänderung } & \multirow[t]{2}{*}{ Statement } & \multicolumn{4}{|c|}{ Änderungen der Bewertung } & \multicolumn{4}{|c|}{ Bewertungen Vorher } & \multicolumn{4}{|c|}{ Bewertungen Nachher } \\
\hline & & V & sig & $\mathrm{L}$ & sig & V & $\mathrm{L}$ & $\Delta$ & sig & V & $\mathrm{L}$ & $\Delta$ & sig \\
\hline \multirow[t]{4}{*}{ Keine Änderungen } & $1 \mathrm{~A}$ & 0,36 & 0,22 & 0,15 & 0,38 & 6,92 & 8,15 & 1,23 & 0,00 & 7,28 & 8,31 & 1,03 & 0,02 \\
\hline & $2 \mathrm{~B}$ & 0,09 & 0,91 & 0,19 & 0,57 & 5,70 & 6,54 & 0,84 & 0,22 & 5,79 & 6,35 & 0,55 & 0,49 \\
\hline & $4 \mathrm{~B}$ & 0,20 & 0,27 & 0,15 & 1,00 & 7,76 & 8,46 & 0,70 & 0,05 & 7,96 & 8,62 & 0,65 & 0,02 \\
\hline & $5 A$ & 0,27 & 0,28 & 0,12 & 0,50 & 8,04 & 8,50 & 0,46 & 0,15 & 8,31 & 8,62 & 0,31 & 0,18 \\
\hline \multirow[t]{2}{*}{ Änderungen der Landwirte } & 1B & 0,04 & 0,94 & 1,23 & 0,01 & 4,30 & 2,04 & 2,27 & 0,00 & 4,35 & 3,27 & 1,08 & 0,08 \\
\hline & $3 A$ & 0,08 & 0,56 & 0,38 & 0,04 & 7,50 & 7,35 & 0,15 & 0,99 & 7,42 & 7,73 & 0,31 & 0,37 \\
\hline \multirow[t]{2}{*}{ Änderungen der Verbraucher } & $3 B$ & 1,40 & 0,01 & 0,15 & 0,55 & 6,40 & 8,12 & 1,72 & 0,00 & 7,80 & 8,27 & 0,47 & 0,21 \\
\hline & $5 B$ & 1,51 & 0,00 & 0,01 & 0,95 & 5,25 & 7,68 & 2,43 & 0,00 & 6,76 & 7,69 & 0,93 & 0,12 \\
\hline \multirow[t]{2}{*}{ Änderungen beider Gruppen } & $2 \mathrm{~A}$ & 2,02 & 0,00 & 0,92 & 0,01 & 7,58 & 2,96 & 4,62 & 0,00 & 5,57 & 3,88 & 1,68 & 0,00 \\
\hline & $4 \mathrm{~A}$ & 2,37 & 0,00 & 0,81 & 0,03 & 7,87 & 3,96 & 3,91 & 0,00 & 5,50 & 4,77 & 0,73 & 0,29 \\
\hline
\end{tabular}

Die Tabelle zeigt die Mittelwerte der Zustimmung zu den Statements ( $1=$ stimme überhaupt nicht zu bis $9=$ stimme voll und ganz zu) für die Verbraucher (V) und die Landwirte (L) vor und nach gemeinsamen Gruppendiskussionen, Unterschiede $(\Delta)$ zwischen Verbrauchern und Landwirten mit entsprechenden exakten 2-seitigen Signifikanzniveaus (sig) durch Mann-Whitney-U-Test und Änderungen der Bewertungen mit entsprechenden exakten 2-seitigen Signifikanzniveaus (sign.) durch Wilcoxon-Vorzeichen-Rang-Test

haben Anspruch auf Fürsorge und Verantwortung durch den Menschen“ und 4B: „Nutztiere sollen behandelt werden, wenn sie krank sind“. Diese Grundeinstellungen sind gleichzeitig bei beiden Gruppen so fest verankert, dass sie auch im Verlauf
Die geringste mittlere Zustimmung beider Gruppen sowohl vor als auch nach den Diskussionen erreicht das Statement 1B: „Zur Landwirtschaft kann

\footnotetext{
${ }_{9}$ Hierbei handelt es sich um Originalwortlaute aus den Gruppendiskussionen.
} 
man sich nur über die Medien informieren“. Landwirte lehnen diese Aussage noch deutlicher ab als Verbraucher, nähern sich nach den Diskussionen allerdings deren Meinung an. Die Rolle der Medien wird von beiden Gruppen kritisch reflektiert, die Verbraucher gehen davon aus, dass Informationen zur Tierhaltung in Medien nicht immer objektiv und neutral sind: "Ich glaube, es ist etwas primitiv, alles in den Medien zu glauben." Gleichzeitig verdeutlichen die Diskussionen, dass vielen Verbrauchern trotz verschiedener Angebote von Seiten der Landwirte wie Hofbesichtigungen oder „Tage der offenen Tür“ Möglichkeiten fehlen, sich faktenbasiert über die Landwirtschaft und die aktuellen Verfahren der Tierhaltung zu informieren. Den Verbrauchern mangelt es an emotionalen und zeitlichen Kapazitäten: „Das ist ja eine Zeitfrage. Wir sind alle berufstätig und wenn wir jetzt alle so um 4, $5 \mathrm{Uhr}$ nach Hause kommen und haben keine Lust mehr uns nochmal ins Auto zu setzen, aufs Land zu fahren und irgendwelche Bauern aufzusuchen und die zu fragen ob das wirklich stimmt oder nicht, was man im Fernsehen sieht.“ Die Bewertungsänderung der Tierhalter zu dem Statement deutet auf Verständnis für dieses verbraucherseitige Dilemma.

Die Statements 3B: „Die Arbeit für Tierhalter wird durch Technik leichter und die Nutztiere können besser versorgt werden“ und 5B: „Nutztiere sollen uns in erster Linie zur Lebensmittelproduktion dienen“ erfahren eine deutlich höhere mittlere Zustimmung der Landwirte als der Verbraucher. $\mathrm{Zu}$ beiden Statements nähern sich die Verbraucher nach den Diskussionen in ihren Einschätzungen den Landwirten an. Im Verlauf der Diskussionen stellen die Landwirte die Vorteile des Technikeinsatzes in der Tierhaltung heraus. Sie verweisen auf die Funktion als Kontrollinstrument für die optimale Versorgung der Tiere: "Aber irgendwo gibt es die Alarmanlage, die plötzlich sagt: Die Fütterung hat gar nicht funktioniert. Da ist was leergelaufen, oder das Wasser ist da nicht angekommen. Da gibt es die Alarmanlage, die irgendwo das Handy des Betreibers anruft, und sagt „guck, da ist was im Stall nicht in Ordnung." Daneben verweisen sie auch auf eine Erhöhung der Betreuungszeit für die Tiere durch den Einsatz von Technik: “(...) alleine dadurch dass man den Mist zum Beispiel nicht mehr mit der Schubkarre rausschieben muss, habe ich ja auch mehr Zeit und kümmere mich ums einzelne Tier." Mit diesen Argumenten scheinen sie die Verbraucher umstimmen zu können. Die Landwirte betonten auch den Unterschied zwischen Nutztieren und Heimtieren und die Funktion, die Nutztiere letztlich für die Verbraucher erfüllen. Die
Verbraucher unterscheiden am Ende der Diskussionen deutlicher zwischen Nutztierhaltung und Heimtierhaltung: "Wenn wir über Nutztiere sprechen, weiß ich, dass das Leben kein Ponyhof ist.”

In der Bewertung der Statements 2A: „Nutztiere sollten bessere Haltungsbedingungen bekommen“ und 4B: „Der Einsatz von Medikamenten in der Tierhaltung ist kritisch zu sehen" besteht vor den Diskussionen ein sichtbarer Dissens zwischen Verbrauchern und Landwirten. Die Verbraucher stimmen beiden Statements deutlich zu, Landwirte lehnen sie eher ab. Nach den Diskussionen nehmen beide Gruppen eine Veränderung ihrer Positionen ein - Verbraucher noch deutlicher als Landwirte, und es kommt zu einer Annäherung der Einschätzungen. In den Diskussionen stellen die Landwirte Vergleiche an und betonen die positiven Entwicklungen für die Tiere: „Die Haltungsbedingungen, wenn man so die letzten Jahre, Jahrzehnte zurückgeht, die haben sich ja deutlich verbessert, [...] weil die Erkenntnisse einfach weiter gegangen sind“. Sie stützen sich auf Gesetze und Richtlinien und verweisen auch auf ihr Berufsethos im Zusammenhang mit der Tierhaltung: „Leitsatz eines jeden guten Landwirtes: Das ist unsere Berufung, unsere Leidenschaft und unsere Pflicht, die Tiere mit Fürsorge und Verantwortung zu versorgen“. Daneben erklären sie den Verbrauchern auch den wirtschaftlichen Druck in der Tierhaltung und beschreiben mögliche dramatische Konsequenzen durch zusätzliche Tierwohlauflagen: „Wenn wir immer mehr fordern, dann bin ich mir ganz sicher, dass dann demnächst die Schweinehaltung in Deutschland einfach ganz verschwindet“. Bei den Verbrauchern erzielen sie offenbar einen deutlichen Vertrauensgewinn und teilweise eine Erleichterung, was sich in der Änderung des Zustimmungsgrades zu dem Statement widerspiegelt. Teilweise bleiben jedoch Zweifel und Unsicherheiten zu den Haltungsverfahren bestehen: „Gibt es keine Alternative dazu?"

In Bezug auf den Medikamenteneinsatz in der Tierhaltung zeigen die Landwirte Verständnis für die Bedenken der Verbraucher, z.B. im Hinblick auf multiresistente Keime. Sie erläutern ihnen die Vorgehensweisen der Behandlung kranker Tiere, einzuhaltende Wartezeiten und Monitoring-Programme der Antibiotikagaben. Am Ende der Diskussionen liegen die Bewertungen der Statements deutlich näher beieinander. Es wird ein gegenseitiges Verständnis für die Interessen und Bedenken der jeweils anderen Gruppe sowie eigene Reflexionen sichtbar. 


\section{Diskussion und Ausblick}

Die Ergebnisse zeigen, dass Landwirte und Verbraucher auf unterschiedliche Wahrnehmungen der Tierhaltung zurückgreifen, dass beide Gruppen gleichzeitig ein grundsätzliches Interesse an der Verbesserung des Tierwohls haben. Dieses Ergebnis lässt sich ethisch als Beleg für die Annahme verstehen, dass die Fähigkeit zu speziesübergreifender Einfühlung und Empathie bevölkerungsgruppenübergreifend bei Landwirten und Verbrauchern weit verbreitet ist. Die Ergebnisse relativieren dabei die Ergebnisse von Te Velde et al. (2002) und Vanhonacker et al. (2008), wonach für Landwirte wichtige Kriterien zur Bewertung der Nutztierhaltung vor allem am Management, an technischen Verbesserungen und an Haltungssystemen an sich festgemacht werden. Auf der anderen Seite zeigen die Ergebnisse, wie wichtig Verbrauchern ein fürsorglicher Umgang mit den Tieren ist (Zander et al. 2013).

Die unterschiedliche Bewertung der heutigen Tierhaltung ist darauf zurückzuführen, dass die Folgen tierwohlfördernder Maßnahmen Landwirte und Verbraucher unterschiedlich stark beträfen. In beiden Gruppen beeinflusst vermutlich eine unterschiedlich stark ausgeprägte Befangenheit die Wahrnehmung und Bewertung. Das ebenfalls unterschiedlich stark ausgeprägte Unwohlsein zum Umgang mit den Tieren dürfte auch damit zusammenhängen, dass Landwirte sich einen „pragmatischen Umgang“ mit ihren eigenen Emotionen gegenüber den Tieren angeeignet haben. Verbraucher dagegen urteilen aus der Distanz. Dass bei Heimtier- und Nutztierhaltung mit zweierlei Maß gemessen wird, verletzt ihr Moral- und Gerechtigkeitsempfinden.

Die Landwirte verfügten berufsbedingt über einen Wissensvorsprung zur Tierhaltung, der sich in einer asymmetrischen Kommunikationsstruktur mit einer dominanten Rolle der Landwirte widerspiegelt. Die Ergebnisse der Gruppendiskussionen zeigen dennoch, dass Verbraucher grundsätzlich gegenüber Landwirten Vertrauen aufbauen können, wenn Informationen glaubwürdig und vertrauenswürdig vermittelt werden. Falls Landwirte jedoch Verbraucher nicht ernst nehmen und versuchen, ihnen ,ihre Wahrheit" zu präsentieren, bewegen sich die Verbraucher in ihrer Einstellung nicht und behalten ihre Ansichten und Zweifel bei.

Verständnis für die Schwierigkeiten der Verbraucher, sich aus eigener Anschauung ein Bild der Nutztierhaltung zu machen, bietet Landwirten die Chance, auf diese Defizite einzugehen und
Möglichkeiten zu suchen, zentrale Aspekte der intensiven Tierhaltung nachvollziehbar zu vermitteln. Auf der anderen Seite bietet der persönliche Kontakt zu den Verbrauchern den Landwirten die Gelegenheit, nicht nur die Kritikpunkte an der Nutztierhaltung sondern auch die Hintergründe und Motivationen der Verbraucher zu verstehen. Durch die Weiterentwicklung alternativer Tierhaltungssysteme, die weniger erklärungsbedürftig sind, könnte verbraucherseitige Akzeptanz einfacher erzielt werden.

Der in dieser Studie verwendete gemischtmethodische Ansatz mit einer Abfrage der Zustimmung zu verschiedenen Statements vor und nach den Diskussionen bietet die Möglichkeit, Diskussionsergebnisse oder Diskussionsergebnisse sichtbar zu machen und zu quantifizieren. Der Vorher-NachherVergleich der Statementbewertungen zeigt 4 verschiedene Arten der Einschätzungsänderung nach den Diskussionen. Verbraucher ändern ihre Einschätzung dabei deutlich stärker als Landwirte. Sie sind offener für neue Informationen und neue Argumente und passen ihre Bewertung eher neu an - möglicherweise auch, weil die sich daraus ergebenden Konsequenzen (noch) nicht mitbedacht werden (müssen). Landwirte, die in ihre Einschätzungen auch die eigenen betrieblichen ökonomischen Zusammenhänge einbeziehen, scheinen mehr daran interessiert, aufzuklären als neue Informationen aus den Diskussionen mit den Verbrauchern zu gewinnen. Deshalb ändern sie ihre Bewertungen nicht so stark wie die Verbraucher.

Das Ziel, der landwirtschaftlichen Tierhaltung wieder zu gesellschaftlicher Akzeptanz zu verhelfen, wird von den politischen Akteuren ebenso wie vom Agrar- und Lebensmittelsektor geteilt. Die Untersuchung der Kommunikation zwischen Landwirten und Verbrauchern hat allerdings gezeigt, dass einer Einigung bisher langfristige ethische Hürden im Wege stehen. Die Studie wirft zwar Licht auf die Frage, inwiefern moderierte Gruppendiskussionen zu einer Annäherung der Einstellungen und Einschätzungen von Verbrauchern und Landwirten führen können. Unklar bleibt aber insbesondere die zeitliche Stabilität der neuen Bewertungen. Daher ist zu prüfen, ob auch andere Formen von Begegnungen zwischen Tierhaltern und Verbrauchern geeignet sind, einen zielführenden Dialog zur Weiterentwicklung und Akzeptanz der landwirtschaftlichen Nutztierhaltung in Deutschland zu fördern. Ohne weitergehende Forschung zur Tierwohl-Kommunikation zwischen Landwirten und Verbrauchern wird 
es auf absehbare Zeit vermutlich keinen Konsens über die „richtigen“ Veränderungen in der Nutztierhaltung geben.

\section{Literatur}

Busch G, Kayser M, Spiller A (2013) „Massentierhaltung" aus VerbraucherInnensicht - Assoziationen und Einstellungen. Aust J Agric Econ Rural Stud 22(1):61-70

Helmle S (2011) Images der Landwirtschaft. Weikersheim

Mayring P (2002) Einführung in die Qualitative Sozialforschung. Eine Anleitung $\mathrm{zu}$ qualitativem Denken. Beltz Verlag, Weinheim und Basel

Spiller A, von Meyer-Höfer M, Sonntag W (2016) Gibt es eine Zukunft für die moderne konventionelle Tierhaltung in Nordwesteuropa? Diskussionsbeitrag 1608, Diskussionspapiere. Department für Agrarökonomie und Rurale Entwicklung, GeorgAugust-Universität Göttingen

Statistische Ämter des Bundes und der Länder (2011) Agrarstrukturen in Deutschland. Einheit in Vielfalt. https://www.destatis.de/DE/Publikationen/Themat isch/LandForstwirtschaft/Landwirtschaftzaehlung/ AgrarstruktureninDeutschland5411203109004. pdf?_blob=publicationFile

Tashakkori A, Teddlie C (1998) Mixed methodology. Combining qualitative and quantitative approaches. Thousand Oaks:Sage

Te Velde H, Aarts N, van Woerkum C (2002) Dealing with ambivalence: Farmers' and consumers' perception of animal welfare in livestock breeding. J Agric Environ Ethics 15:203-219
Vanhonacker F, Verbeke W, van Poucke E, Pieniak Z, Nijs G, Tuyttens F (2012) The concept of farm animal welfare: citizen perceptions and stakeholder opinion in flanders, Belgium. J Agric Environ Ethics 25(1):79-101

Vanhonacker F, Verbeke W, van Poucke E, Tuyttens F (2008) Do citizens and farmers interpret the concept of farm animal welfare differently? Livest Sci 116:126-136

Vierboom C, Härlen I, Simons J (2015) Kommunikation im Perspektivenwechsel - Eine Analyse der Chancen zum Dialog zwischen Landwirten und Verbrauchern. In: Die Landwirtschaft im Spiegel von Verbrauchern und Gesellschaft. Schriftenreihe der Landwirtschaftlichen Rentenbank, Band 21, Frankfurt a.M., S. 97-134

Wildraut C, Plesch G, Härlen I, Simons J, Hartmann M, Ziron M, Mergenthaler M (2015) Multimethodische Bewertung von Schweinehaltungsverfahren durch Verbraucher anhand von Videos aus realen Schweineställen. Forschungsberichte des Fachbereichs Agrarwirtschaft Soest, Nr. 36

WBA Wissenschaftlicher Beirat Agrarpolitik beim BMEL (2015) Wege zu einer gesellschaftlich akzeptierten Nutztierhaltung. Gutachten. Berlin

Zander K, Isermeyer F, Bürgelt D, Christoph-Schulz I, Salamon P, Weible D (2013) Erwartungen der Gesellschaft an die Landwirtschaft. Münster: Stiftung Westfälische Landwirtschaft 


\section{Wahrnehmung der Nutztierhaltung - alles eine Frage der Kommunikation?}

\section{Nadine Gier*, Caspar Krampe*, Peter Kenning}

Lehrstuhl für Betriebswirtschaftslehre, insb. Marketing, Heinrich-Heine-Universität, Universitätsstraße 1, 40225 Düsseldorf, Gebäude 24.21, Deutschland 0211-81-14655

nadine.gier@hhu.de, caspar.krampe@hhu.de

Mit Kommentaren von Lucia A. Reisch, CCMP, Zeppelin Universität Friedrichshafen, Am Seemooser Horn 20, Friedrichshafen, Deutschland, lucia.reisch @zu.de

* Beide Autoren haben zu dem Artikel in gleichem Maße beigetragen.

\section{Einführung}

Ziel der betrieblichen Kommunikationspolitik im Rahmen der marktorientierten Unternehmensführung ist es u.a., Kunden und Verbraucher über die Eigenschaften der angebotenen Produkte oder Services zu informieren (Verbeke \& Ward 2006). Im Kontext der in die Lebensmittelwirtschaft eingebundenen Nutztierhaltung haben dabei neben allgemeinen Angaben - zum Beispiel zur Art, der Herkunft, den ernährungsphysiologischen Werten oder den Verarbeitungseigenschaften - insbesondere Informationen über die mit der Tierhaltung verbundenen Praktiken eine hohe Bedeutung (Roininen et al. 2006; Wille et al. 2016). So spielen, vor dem Hintergrund der aktuellen gesellschaftlichen Diskussion um artgerechte Tierhaltung und der zunehmenden Kritik an der Massentierhaltung, Aspekte der Prozessqualität neben Kriterien der Produktqualität eine übergeordnete Rolle bei Kaufentscheidungen. Um den damit verbundenen Informationsbedarf der Verbraucher zu decken und am Markt höhere Prozessqualitäten zu signalisieren, werden von den Anbietern und Herstellern regelmäßig Kombinationen von Bild- und Textelementen verwendet, deren Wirkungsweise die Wahrnehmung der Verbraucher auf kognitiver und emotionaler Ebene beeinflussen kann (Levin 1987). Es spielt also nicht nur die bewusste Wahrnehmung und Verarbeitung der Information eine Rolle, sondern ebenso die unbewusste Wirkung der Darstellungsweisen, sogenannten Frames (Levin 1987; Levin et al. 1998).
Framingeffekte können auf unterschiedliche Weise hervorgerufen werden. Ein Beispiel wären veränderte Umgebungselemente, wie beispielsweise Bildelemente am Point-of-Sale (PoS), die unterschiedliche Assoziationen der Verbraucher beim Kauf von tierischen Produkten aktivieren. Diese können einen positiven oder negativen Effekt auf das Kaufempfinden bzw. -verhalten ausüben (Chowdhury et al. 2008). Lange Zeit jedoch konnten diese, dem Verbraucher meist unbewussten Einflüsse, kaum empirisch erfasst werden. Demzufolge konnte der zugrundeliegende Mechanismus innerhalb der sogenannten „Black Box“ oft nur theoretisch beschrieben werden (Kenning \& Plassmann 2005). Mithilfe von neuroökonomischen Analysen erscheint es nun aber möglich, die mit dem hier interessierenden Verbraucherverhalten verbundenen, oft unbewussten neuralen Prozesse direkt, das heißt in vivo $\mathrm{zu}$ beobachten und hinsichtlich ihrer Verhaltensrelevanz einzuordnen.

Die damit angesprochene Consumer Neuroscience (Kenning 2014) hat in einigen Fällen dazu beigetragen, eine erhöhte Varianzaufklärung des Verbraucherverhaltens zu erlangen (Hubert \& Kenning 2008; Kenning et al. 2017; Kenning \& Plassmann 2005; Kosslyn 1999; Plassmann et al. 2015). Darauf aufbauend könnten zukünftige Informationsstrategien und -maßnahmen entwickelt werden, die eine effektivere und gegebenenfalls effizientere Kommunikation und Verbraucherinformation im Kontext der Nutztierhaltung ermöglichen.

Der vorliegende Beitrag stellt die Ergebnisse einer SocialLab-Studie vor, bei der 2 Verfahren der Consumer Neuroscience eingesetzt wurden, um zu untersuchen, wie kommunikative Maßnahmen direkt am PoS zum Thema Tierwohl wahrgenommen und damit ggf. kaufentscheidend werden können.

\section{Methoden und Ergebnisse}

Um die Wirkungsweise verschiedener Kommunikationsmaßnahmen auf die Verbraucherwahrnehmung im Bereich der Nutztierhaltung zu untersuchen, wurden im Rahmen des SocialLab-Projektes 2 sogenannte „bildgebende“ Methoden verwendet. Zum einen handelte es hierbei um die funktionale Magnetresonanztomografie (fMRT) (Dimoka 2010), zum anderen um die in vielerlei Hinsicht innovative funktionale Nah-Infrarot Spektroskopie (fNIRS) (Kopton \& Kenning 2014; Krampe et al. 2016). Die Studien und die Ergebnisse sollen im Folgenden kurz skizziert werden. 


\section{1 fMRT-Studie}

Ziel der fMRT-Studie war es zu untersuchen, ob es möglich ist, mit Hilfe der Theorie des regulatorischen Fokus (Crowe \& Higgins 1997) neurophysiologische und oftmals unbewusst ablaufende Wahrnehmungsprozesse im Kontext verschiedener Haltungsmethoden der Nutztierhaltung zu interferieren. Diese Frage ist deswegen bedeutsam, weil es bis dato unklar ist, ob kommunikative Maßnahmen überhaupt einen Einfluss auf die Einstellung der Verbraucher in diesem Bereich haben können oder ob die entsprechenden Reaktionen nicht vielmehr quasi reflexhaft ablaufen und im Gehirn mehr oder weniger „fest verdrahtet“ sind. Um eine Antwort auf diese Frage zu finden, wurde nach Freigabe des Studiendesigns durch eine Ethikkommission eine fMRTAnalyse mit 29 Probandinnen und Probanden (Alter $\mathrm{M}=41,45$ Jahre, $\quad \mathrm{SD}=10,83 ; \quad 14 \quad$ weiblich) durchgeführt.

Im Vorfeld der MRT-Messungen wurden verschiedene Haltungsmethoden der Nutztierhaltung entsprechend der regulatorischen Fokus Theorie als Informationen differenziert formuliert. Dabei wurden two Foki unterschieden: Der Promotionsfokus ist dadurch gekennzeichnet, dass er Erfolge einer Maßnahme in den Vordergrund stellt. Im Gegensatz dazu ist es Merkmal des Präventionsfokus', dass dieser Sicherheits- und Schutzaspekte thematisiert. Aufgabe der Probanden war es, sich die entsprechend formulierte Information aufmerksam durchzulesen und im Nachgang eine bildlich dargestellte Haltungsmethode zu betrachten und anschließend zu bewerten (Abb. 1). In den Analysen wurde die Gehinaktivität während des Informationszeitraums und der Bildwahrnehmung zwischen den beiden Formulierungsarten analysiert.

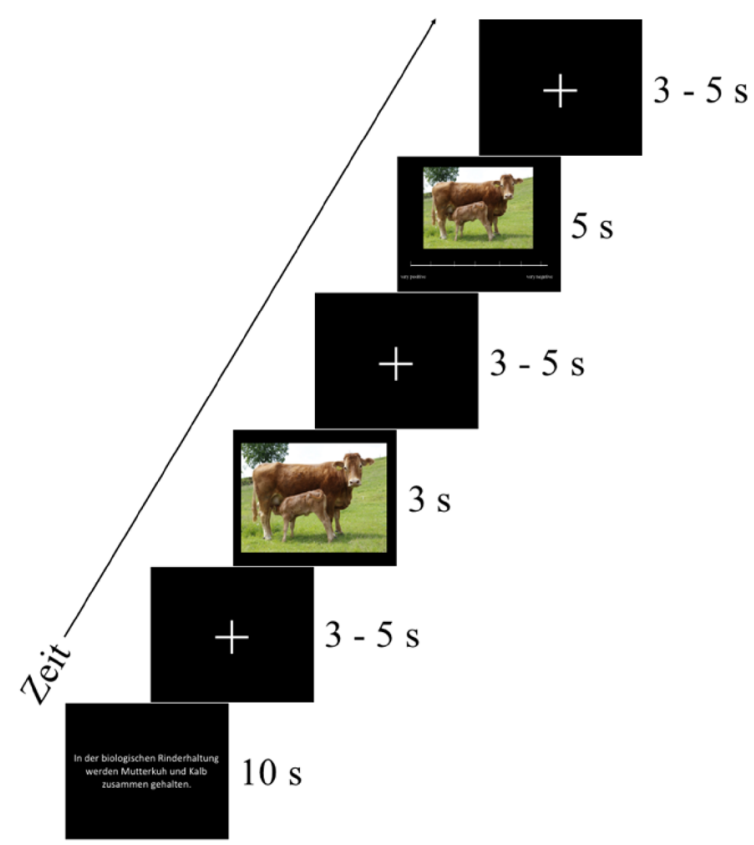

Abb. 1 Versuchssequenz der experimentellen fMRT-Aufgabe. Eine Versuchssequenz bestand aus drei Abschnitten, die durch eine zeitlich randomisierte Pause getrennt waren (,jitter“). In dem Informationsabschnitt wurde zunächst die Information über eine Haltungsmethode genannt. Anschließend wurde das dazugehörige Bild angezeigt. Schließlich sollte der Proband diese Haltungsmethode bewerten

Die ersten Ergebnisse der neuralen fMRT-Analyse zeigten, dass insbesondere der ventromediale präfrontale Kortex (vmPFC) immer dann eine stärkere Aktivität aufwies, wenn die Information im Promotionsfokus im Kontrast zum Präventionsfokus formuliert war (Abb. 2). Demzufolge erzeugt eine Information, welche sich auf Erfolge, Errungenschaften und Verbesserungen fokussiert, eine stärkere neurale Reaktion in Hirnregionen, welche mit emotionalen Bewertungsprozessen assoziiert 
sind. Frühere Studien der Consumer Neuroscience haben zudem gezeigt, dass der vmPFC eine zentrale Rolle für das Bewertungssystem sowie das Kaufverhalten spielt (Bartra et al. 2013; Enax et al. 2015; Plassmann \& Weber 2015). Dies bedeutet im Hinblick auf die Forschungsfrage zum einen, dass eine Beeinflussung neuraler Prozesse durch eine zieladäquate Darstellungsform der jeweiligen Information offenbar möglich ist. Zum anderen zeigt sich, dass Informationen beziehungsweise Darstellungsformen, die Verbesserungen beziehungsweise Erfolge einer Maßnahme in den Vordergrund stellen, eine erhöhte neurale, subjektiv-emotionale Wertung erfahren als sicherheits- und schutz-orientierte Informationen. Betrachtet man zudem die zentrale Rolle von Emotionen im Konsumverhalten insgesamt, so scheinen entsprechend modifizierte Informationen die Fähigkeit zu besitzen, das Konsumverhalten auf neuraler Ebene signifikant zu beeinflussen. Somit kann die Art und Weise der Kommunikation die Wahrscheinlichkeit erhöhen, dass Informationen zur Tierhaltung handlungsrelevante Implikationen für die Verbraucher haben. die Akzeptanz haben können. So kann diese Studie dazu beitragen, differenzierte, akzeptanzfördernde Kommunikation für Verbraucher zu entwickeln.

\section{2 fNIRS-Studie}

Aufbauend auf diesen Ergebnissen wurden ergänzende fNIRS-Studien durchgeführt. Die mobile fNIRS bietet hierbei einen innovativen Ansatz die neuralen Prozesse, ähnlich dem Prinzip der fMRT, mittels Lichtimpulsen zu quantifizieren (Kopton \& Kenning 2014). Durch die mobile Einsetzbarkeit können neurale Prozesse und assoziiertes Konsumentenverhalten in einem naturalistischen Umfeld bemessen werden. Somit bestand das Ziel dieser Studien darin, zu prüfen, welche neuralen Prozesse am PoS ablaufen, wenn Verbraucher eine „echte“ nutztierhaltungsrelevante Kaufentscheidung unter realitätsnahen Bedingungen treffen. Parallel hierzu sollte zudem die Validität der mobil einsetzbaren fNIRS untersucht werden. Hierbei zeigte sich zunächst, dass die mobile fNIRS eine valide neurowissenschaftliche Methodik im Forschungsfeld der "Consumer Neuroscience“ dar-
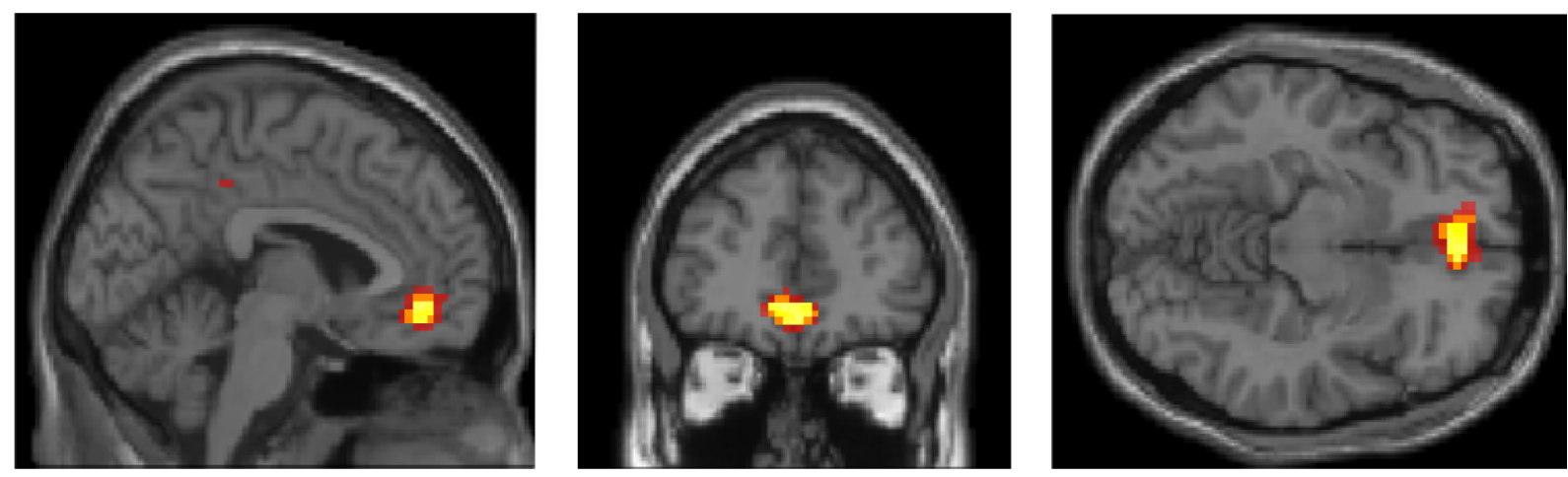

Abb. 2 Neuraler Regulatorischer Fokus Effekt. Darstellung der signifikanten Unterschiede zwischen den auf der regulatorischen Fokustheorie basierten Informationen (Promotions-

Betrachtet man diese Ergebnisse bezugnehmend auf das übergeordnete Ziel der Wirkung unterschiedlicher Darstellungsvarianten der Tierhaltungsverfahren auf die (implizite) Wahrnehmung und die möglicherweise daraus resultierende gesellschaftliche Akzeptanzgewinnung, so lässt sich feststellen, dass die untersuchten Darstellungsweisen die neurale emotional-subjektive Wertung signifikant beeinflussen. In weiteren tiefergehenden Analysen wird nun nach Tierhaltungsmethoden differenziert, da Werte und a priori Einstellungen einen Einfluss auf den Effekt von Kommunikation auf fokus $>$ Präventionsfokus) in sagittaler, koronaler und axialer Ansicht (von links nach rechts), (Peak Voxel: MNI-Koordinaten -3 44 -10; rot $\mathrm{p}<.001$; gelb $\mathrm{p}<0.0001$; weiß $\mathrm{p}<0.00001$ )

stellt (Krampe et al. 2016). Aufbauend auf diesen ersten Forschungsergebnissen wurde eine zweite Studie konzipiert, welche auf die Datenerhebung am PoS fokussierte und somit die mobile Einsetzbarkeit der fNIRS in einer innovativen Feldstudie prüfen sollte. Hierzu wurden außerhalb der Öffnungszeiten in einem Lebensmittelmarkt über der Selbstbedienungstheke für Fleischwaren entweder biologischoder konventionell-orientierte Tierhaltungskommunikationsmaßnahmen (TKM), die als situative Frames fungierten, platziert. Um die neurale Reaktion der Probandinnen ( $\mathrm{n}=18$; Alter durchschnittlich 41 Jahre, 
$\mathrm{SD}=7,96)$ auf die dementsprechend veränderten Marktaufbauten zu erfassen, wurden diese mit einen mobilen fNIRS- und Eye-Tracking-Gerät ausgestattet. Danach wurden sie gebeten, einem vorgegebenen Einkaufsweg zu folgen und einen Einkauf zu tätigen.

Im Rahmen der Datenanalyse wurde die Gehirnaktivität der beiden TKM (biologisch- und konventionell-orientierte TKM) kontrastiert (Abb. 3). Die Ergebnisse der fNIRS Datenanalyse zeigten bei einer statistisch-liberalen Auswertung $(\mathrm{p}<0.1)$, dass insbesondere Regionen des orbitofrontalen Kortex (OFC) sensitiv für Veränderungen der TKM sind. So ist die neurale OFC-Hirnaktivität im Kontrast zu konventioneller TKM bei biologisch-orientierter TKM erhöht. konventionell-orientiert). Dies lässt vermuten, dass die situative Präsentation der TKM im Markt, also am PoS, den entscheidenden Einfluss auf die neurale Reaktion der Kunden und den damit verbundenen oft unbewussten Kaufentscheidungsprozess hat. Somit scheint das Entscheidungsumfeld (also der situative Frame), in welche die TKM platziert wird, eine zentrale Rolle einzunehmen. Dieser Aspekt wird in der verbraucherpolitischen Diskussion über in anderer Hinsicht optimierte Informations- und Kommunikationsinstrumente oft übersehen und unterstreicht noch einmal die zentrale Rolle des Handels, der das Entscheidungsumfeld maßgeblich gestaltet (vgl. hierzu auch den Beitrag „Standards, Hindernisse und Wünsche in der Nutztierhaltung -
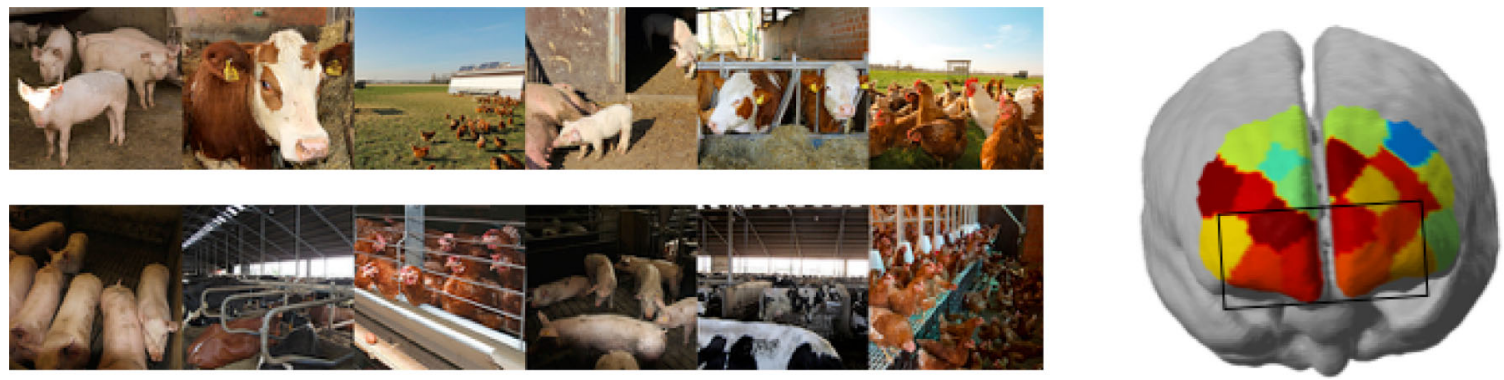

Abb. 3 Neurale Wirkung von TKM. Darstellung des Kontrastes biologisch-orientierte vs. konventionell-orientierte TKM $(n=18$; dunkel rot $\mathrm{p}<0.1$; Regionen des OFC in schwarzer Box) (rechts).

Ergänzend zu den fNIRS-Daten wurden über einen Zeitraum von 6 Wochen die mit der TKM assoziierten Abverkaufszahlen pseudo-randomisiert ermittelt. Hierbei zeigte sich für die biologisch-orientierte TKM ein signifikant höherer durchschnittlicher Fleischwaren-Wochenumsatz pro Kunde (Wochenumsatz Fleischware/Anzahl Kunden; $t(5)=2,65, p<0.05$ ). Betrachtet man beide Befunde im Zusammenhang, so lässt sich schlussfolgern, dass biologisch-orientierte TKM zu einer höheren neuralen Reaktion im OFC führt. Dies wiederum weist auf eine erhöht-aktivierte, subjektive Bewertung der Probanden hin, welche offenbar verhaltensrelevante Auswirkungen auf das Einkaufsverhalten der Kunden hat.

Des Weiteren wurden die TKM mit Hilfe der fMRT im Labor untersucht, um Befunde zur umfassenderen Lokalisierung der neuralen Aktivität zu ergänzen. Die Resultate zeigen jedoch überraschenderweise keine signifikanten Aktivitätsunterschiede zwischen den beiden verwendeten TKM (biologisch- und
Links oben: biologisch-orientierte TKM; links unten: konventionellorientierte TKM

Die Perspektive des Handels“ im vorliegenden Heft). In Bezug auf das Ziel der Identifizierung der mit dem relevanten Verhalten neuropsychologischen Prozesse zeigt die fNIRS-Technologie somit eine höhere externe ökologische Validität und gibt wichtige Hinweise, die in einer weniger biotischen Studienanlage nicht gewonnen werden konnten. Konkret zeigt sich, dass kaufentscheidende Phänomene oft erst am PoS entstehen - ein Resultat, dass die verhaltensökonomische Konsumforschung als „konstruierte Präferenzen“ (constructive preferences) bezeichnet und mit der so genannten Query Theory (also „Abfrage-Theorie“) begründet (Johnson, Steffel, \& Goldstein, 2005). Dies unterstreicht in methodischer Hinsicht noch einmal die besondere Bedeutung der mobilen fNIRS und verdeutlicht theoretisch die Rolle sogenannter „exogener Präferenzen“ in den entsprechenden Entscheidungsprozessen. 


\section{Ausblick und Implikationen}

Betrachtet man die dargestellten Forschungsergebnisse bezugnehmend auf das übergeordnete Ziel, die neuropsychologische Wirkung unterschiedlicher Darstellungsvarianten der Tierhaltungsverfahren auf die Wahrnehmung und die möglicherweise daraus folgende gesellschaftliche Akzeptanzgewinnung besser zu verstehen, so lässt sich feststellen, dass die untersuchten Darstellungsweisen die impliziten neurophysiologischen Prozesse signifikant beeinflussen. Insbesondere scheint die oftmals implizite Wirkungsweise von Darstellungsvarianten der Tierhaltungsverfahren bedeutsam zu sein. Des Weiteren spielt der Präsentationsrahmen - das Framing - eine entscheidende Rolle in der (impliziten) Kommunikationswahrnehmung und -verarbeitung der Verbraucher. Es ist daher wichtig, diese Aspekte Darstellung und Kontext - bei der Gestaltung von Kommunikationen im Bereich der Nutztierhaltung künftig noch stärker zu beachten. Und auch wenn die Ergebnisse der vorgestellten Studien vorläufig sind und erste Hinweise geben, in welche Richtung weiter geforscht werden sollte, so wird doch erkennbar, dass die Frage nach dem „Wie?“ bei der Gestaltung von Verbraucherinformationen auch in diesem Bedarfsfeld künftig von Bedeutung sein wird.

Die Ergebnisse bestärken zudem die zunehmend in der sozialwissenschaftlichen Konsumforschung gewonnene Erkenntnis, dass individuelle Faktoren wie Wissen, Einstellungen und Handlungsintentionen zwar durchaus bedeutend sind für die Kaufentscheidung, dass jedoch der unmittelbare Entscheidungskontext der letztlich ausschlaggebend ist.

Die Ausführungen sind zum Teil das Ergebnis einer umfassenden Diskussion mit Kollegen, denen wir zu Dank verpflichtet sind. Er gilt allen Projektpartnern des Verbundprojektes „SocialLab - Nutztierhaltung im Spiegel der Gesellschaft“, insbesondere den Kollegen des Privaten Forschungs- und Beratungsinstituts für angewandte Ethik und Tierschutz INSTET gGmbH (Prof. Dr. Jörg Luy) und der Rheinischen Friedrich-Wilhelm-Universität Bonn (Prof. Dr. Monika Hartmann). Zudem danken wir Frau Anja Westphal und Frau Carina Hoffmann für die tatkräftige Unterstützung im Rahmen der Datenerhebung. Die Förderung des Vorhabens erfolgte aus Mitteln des Bundesministeriums für Ernährung und Landwirtschaft (BMEL) aufgrund eines Beschlusses des deutschen Bundestages. Die Projektträgerschaft erfolgte über die Bundesanstalt für Landwirtschaft und Ernährung (BLE) im Rahmen des Programms zur Innovationsförderung (FKZ: 2817203413).

\section{Literatur}

Bartra O, McGuire J T, Kable J W (2013) The valuation system: a coordinate-based meta-analysis of BOLD fMRI experiments examining neural correlates of subjective value. NeuroImage 76:412-427. https:// doi.org/10.1016/j.neuroimage.2013.02.063

Chowdhury RMMI, Olsen GD, Pracejus JW (2008) Affective responses to images in print advertising: affect integration in a simultaneous presentation context. J Advert 37(3):7-18. https://doi.org/10.2753/ JOA0091-3367370301

Crowe E, Higgins E T (1997) Regulatory focus and strategic inclinations: Promotion and prevention in decision-making. Org Behav Hum Dec Process 69(2):117-132. https://doi.org/10.1006/obhd.1996. 2675

Dimoka A (2010) What does the brain tell us about trust and distrust? Evidence from a functional neuroimaging study. MIS Quart 373-396.

Enax L, Krapp V, Piehl A, Weber, B (2015). Effects of social sustainability signaling on neural valuation signals and taste-experience of food products. Front Behav Neurosci 9:247. https://doi.org/10. 3389/fnbeh.2015.00247

Higgins (1997) Beyond pleasure and pain. Am Psychol 52(12):1280-300. https://doi.org/10.1037/0003-066X. 52.12 .1280

Hubert M, Kenning P (2008) A current overview of consumer neuroscience. J Consum Behav 7:272-292. 10.1002/cb

Johnson E, Steffel M, Goldstein D G (2005) Making better decisions: from measuring to constructing preferences. Health Psychol 24(4):S17-S22.

Kahneman D (2012) Schnelles Denken, langsames Denken.

Kenning P, Oehler A, Reisch L A, Grugel C (2017) Verbraucherwissenschaften: Rahmenbedingungen, Forschungsfelder und Institutionen, SpringerVerlag.

Kenning P, Plassmann H (2005) NeuroEconomics: An overview from an economic perspective. Brain Res Bull 67(5):343-354. https://doi.org/10.1016/j. brainresbull.2005.07.006

Kenning P (2014) Consumer Neuroscience: Ein transdisziplinäres Lehrbuch. Kohlhammer Verlag

Kenning P, Plassmann H (2005) NeuroEconomics: An overview from an economic perspective. Brain Res Bull 67(5):343-354 
Krampe C, Strelow E, Kenning P (2016) Functional near-infrared spectroscopy (Fnirs): a new tool for consumer research? ACR North American Advances

Kosslyn S M (1999) If neuroimaging is the answer, what is the question? Philos Trans R Soc Lond Ser B Biol Sci 354(1387):1283-1294. https://doi.org/10. 1098/rstb.1999.0479

Roininen K, Arvola A, Lähteenmäki L (2006) Exploring consumers' perceptions of local food with two different qualitative techniques: Laddering and word association. Food Qual Prefer 17(1):20-30

Levin I P (1987) Associative effects of information framing. Bull Psychonomic Soc 25(2):85-86. https:// doi.org/10.3758/BF03330291

Levin I P, Schneider S L, Gaeth G J (1998) All frames are not created equal: a typology and critical analysis of framing effects 76(2):149-188
Plassmann H, Venkatraman V, Huettel S, Yoon C (2015) Consumer neuroscience: applications, challenges, and possible solutions. J Mark Res 8:427-435

Plassmann H, Weber B (2015) Individual differences in marketing placebo effects: evidence from brain imaging and behavioral experiments. J Mark Res 8:1-18. https://doi.org/10.1509/jmr.13.0613

Verbeke W, Ward R W (2006) Consumer interest in information cues denoting quality, traceability and origin: an application of ordered probit models to beef labels. Food Qual Preference 17(6):453-467. https://doi.org/10.1016/j.foodqual. 2005.05.010

Wille S, Ermann M, Spiller A (2016) Informationsbedürfnis beim Kauf von regionalem Schweinefleisch: Ein Experiment auf Basis der Information-Display-Matrix. Tagungsband 2016, 97 


\section{Zur Konzeption eines}

Verbraucherinformationssystems als Ergänzung oder Alternative? - zum klassischen

Informationslabel

Nadine Gier ${ }^{1}$, Caspar Krampe ${ }^{1}$, Lucia A. Reisch ${ }^{2}$, Peter Kenning ${ }^{1}$

${ }^{1}$ Lehrstuhl für Betriebswirtschaftslehre, insb. Marketing, Heinrich-Heine-Universität, Universitätsstraße 1, 40225 Düsseldorf, Gebäude 24.21

${ }^{2}$ Zeppelin Universität Friedrichshafen, Am Seemooser Horn 20, Friedrichshafen

nadine.gier@hhu.de

\section{Hintergrund und Zielsetzung}

Eine wesentliche Problematik moderner Volkswirtschaften bilden die oft durch arbeitsteilige Prozesse und entsprechend ausdifferenzierte Wertschöpfungsketten induzierten Informationsasymmetrien zwischen den Anbietern und Nachfragern einer Leistung. Beim Thema „Tierwohl“ kommt hinzu, dass dies eine Vertrauenseigenschaft („Credence Attributes ${ }^{*}$ ) des Produktes darstellt, deren Ausprägung vom Verbraucher, beispielsweise beim Produkt „Fleisch“, am Point-of-Sale (PoS) kaum festgestellt werden kann. ${ }^{10}$ Die Informationsökonomik hat auf dieses Marktversagen reagiert und verschiedene Ansätze entwickelt, solche Informationsasymmetrien zu reduzieren. Die klassische Antwort der Verbraucherpolitik ist das „Signaling“ mit Hilfe von „Labels“ oder „Siegeln“. Im Kern sollen diese dem Verbraucher auf den ersten Blick, leicht verständlich und verlässlich eine - mehr oder weniger - bestimmte Qualität signalisieren (Eberle et al. 2011; Olaizola und Corcoran 2003; Reisch 2003).

Mit diesem Ansatz verbinden sich mehrere Vorteile: So bieten Label zum Beispiel in der Lebensmittelwirtschaft die Möglichkeit, den Verbraucher direkt am PoS über produktspezifische Vertrauenseigenschaften, wie bspw. Tierwohl- oder Bio-Aspekte, zu informieren (Eberle et al. 2011; Janssen und Hamm 2012; Olaizola und Corcoran 2003; Reisch 2003). Dabei lassen sich Label-Qualitäten in unterschiedlicher Breite und Tiefe definieren. Während beispielsweise "gentechnikfrei“ ein Prozessattribut betrachtet, behandeln Bioqualitäts-

\footnotetext{
$\overline{10}$ In wenigen Fällen (wie bei der „gläsernen Produktion“) bieten Anbieter die Möglichkeit, bspw. per QR Code auf der Packung direkt in den Stall zu schauen oder sogar sein eigenes Jungtier aufzuziehen und verarbeiten $\mathrm{zu}$ lassen. Selbst in diesen Fällen bleiben jedoch Vertrauensmerkmale bestehen.
}

oder QS-Siegel umfassendere Merkmale. Diese setzen dabei durchaus auf Halo-Effekte, also auf Qualitätsvermutungen, die über die eigentliche Qualität des Produktes hinausgehen.

Recht schnell stoßen Label jedoch an ihre Grenzen und erzeugen oftmals unerwünschte Nebeneffekte (Eberle et al. 2011; Franz et al. 2010). So können Verbraucher beim alltäglichen Einkauf durch die Vielzahl an Labeln, verbunden mit einem geringen Involvement, verwirrt und überfordert werden (Roosen et al. 2003; Verbeke 2005, 2008). Gerade im Bereich der Nutztierhaltung gibt es eine Flut an Labeln, so dass bisweilen je nach Qualitätsprüfung dasselbe Produkt mit mehreren, unterschiedlichen Labeln gekennzeichnet werden kann. So fordert u.a. auch der Bundesverband der Verbraucherzentrale mehr Transparenz und eindeutige Kennzeichnungen im Bereich des Tierwohls in der Nutztierhaltung (VZBV 2017). Diese Vielfalt und gelegentliche Inkonsistenz hat Auswirkungen auf die Glaubwürdigkeit der einzelnen Label und zeigt, dass das Labelsystem in seiner jetzigen Form wohl einen „abnehmenden Grenznutzen“ hat (Verbraucherkommission Baden-Württemberg 2011). Die Verfügbarkeit label-induzierter Information stellt heute somit kein Maximierungs-, sondern ein lokales Optimierungsproblem dar, das in der Praxis auf erhebliche Probleme stößt (Kenning et al. 2017). Vor diesem Hintergrund ist es Ziel des im Folgenden zu skizzierenden Forschungsprojektes, Hinweise für die zukünftige effektive und, nach Möglichkeit, effiziente Gestaltung der Kommunikation von Verbraucherinformationen zu geben. Darauf aufbauend sollen Politik- und Kommunikationsempfehlungen für die gesetzliche und privatwirtschaftliche Umsetzung von Kennzeichnungsmaßnahmen abgeleitet werden. Um dieses Ziel zu erreichen, werden verschiedene Ansätze der Verbraucherinformation diskutiert und Erkenntnisse aus bisherigen Forschungsarbeiten genutzt, um bestehende Instrumente zu optimieren bzw. zu ergänzen oder Alternativen zum bisherigen System der Informationsökonomik durch klassische Label zu entwickeln.

\section{Methodik}

Um das Themenfeld der Verbraucherinformation zunächst phänomenologisch zu durchdringen, wurden im ersten Schritt im Kontext der Nutztierhaltung der Informationsstand, die Informationsbeschaffung, sowie der Informationseinfluss von und zu Verbrauchern mittels einer Literaturrecherche und einer flankierenden Fokusgruppe untersucht. Die aus der qualitativen Sozialforschung stammende Methode 
der Fokusgruppe (Krueger und Casey 2014) ermöglicht es, den Informationsprozess aus Sicht der Verbraucher zu begreifen und mögliche Anschlusskriterien für die Informationsbereitstellung und -beschaffung $\mathrm{zu}$ identifizieren. Im konkreten Projektfall wurden mit Hilfe einer moderierten Diskussion 9 Verbraucher/innen eingeladen, sich über die Thematik der Informationskommunikation im Bereich der Nutztierhaltung auszutauschen und diese zu diskutieren. Anhand der daraus gewonnenen Erkenntnisse konnten unterschiedliche Bedürfnisse und Motive der Verbraucher an Informationsinhalten und zur Informationsbeschaffung zum Thema Nutztierhaltung unterschieden werden. Daraus abgeleitet wurden in einer tiefergehenden Literaturrecherche Alternativen zu den heutigen Angeboten der Verbraucherinformation gesucht, welche bereits durch Studien erste Hinweise auf ihre Effektivität geben oder in ähnlicher Form in anderen Bereichen genutzt werden.

\section{Ausgewählte Ergebnisse}

Im Rahmen der Fokusgruppe zeigte sich, dass retrospektiv wahrgenommene Informationen lediglich auf das Herkunftsland sowie quantitative Kennzahlen wie Haltbarkeit, Preis und Gewicht, beschränkt waren und insgesamt eher undifferenziert und oberflächlich erinnert wurden. Informationen zur Haltungs- und Schlachtungsweise sowie zur Futterbzw. Medikamentenzugabe wurden zwar in der Fokusgruppe als wünschenswerte Information genannt; sie scheinen jedoch in empirischen Studien bei der Kaufentscheidung kaum eine Rolle zu spielen (Andersen 2011; Harper und Henson 2001; Olaizola und Corcoran 2003). Des Weiteren schienen die Verbraucher nicht grundsätzlich, sondern eher ausnahmsweise bewusst und aktiv nach ausführlicheren Informationen zu suchen und nur gewisse Angaben je nach individuellem Involvement und persönlicher Situation - als relevant einzuordnen. Auch Label schienen hier wenig zu bewirken, da es oft keinen entsprechenden Informationsbedarf gibt. Als Konsequenz wurden Label von der Fokusgruppe überwiegend als unverständlich beschrieben und ihre Fülle und Vielfalt eher als lästig empfunden. Gleichwohl teilten die Verbraucher die Meinung, dass Labels die einzige Möglichkeit böten, sich über die Produkte und deren Eigenschaften am PoS zu informieren. So wurde die Verpackung als einzige Informationsoberfläche angesehen, die neben dem subjektiven Aussehen des eigentlichen Produktes Aufschluss über dessen Merkmale geben könne. Im Gegensatz zu diesen grundsätzlichen Aussagen schien das Informationsbedürfnis bei den Verbrauchern in der Fokusgruppe nur dann erhöht zu sein, wenn durch Skandale - wie BSE in Rindfleisch oder die kritische Berichterstattung über unzureichende Tierhaltungsverfahren - der sorgenfreie Konsum von Fleischwaren eingeschränkt wird. In diesem Fall werden aus „vertrauenden“ Verbrauchern, die sich durch eine durchaus rationale Naivität auszeichnen, offenbar „verantwortungsvolle“ Verbraucher, die ein entsprechend gesteigertes Informationsbedürfnis haben (Micklitz et al. 2010; Wobker et al. 2012). So gaben die Verbraucher an, während solcher Krisenzeiten, welche auch moralischer Natur sein können, einen erhöhten Informationsbedarf $\mathrm{zu}$ haben und vermehrt auf Label zu achten oder alternativ auf den „Metzger des Vertrauens“ zurückzugreifen.

Im Ergebnis zeigt sich, dass Label eher eine situative Relevanz haben und je nach Kontext und Involvement selektiv durch die Verbraucher wahrgenommen werden. Zwar werden Label eher wahrgenommen und können kaufentscheidungswirksam sein, wenn sie einfach und intuitiv gestaltet und auf der Vorderseite der Verpackung angebracht sind, und durch entsprechende Kommunikationskampagnen begleitet werden (Grunert 2002; Padilla et al. 2007), jedoch sind sie während eines gewöhnlichen Einkaufs eher wenig relevant. In (moralischen) Krisenzeiten hingegen gewinnen sie an Bedeutung, weisen dann aber oftmals zu wenig Informationen auf, so dass ergänzende Informationsquellen, die oft mit Personenvertrauen ausgestattet sind, hinzugezogen werden. Dieses Ergebnis stimmt mit der aktuellen Forschungslage überein, nach der ein individueller, möglichst personalisierter, zeitlich-flexibler und differenzierter Informationsfluss den Verbrauchern in ihren Entscheidungen helfen kann, ohne diese dabei zu überfordern (de Jonge et al. 2015; Eberle et al. 2011; Kenning et al. 2017, S.289; Reisch 2003; Weinrich und Spiller, 2016). Die folgende Abbildung verdeutlicht diesen Zusammenhang grafisch. 


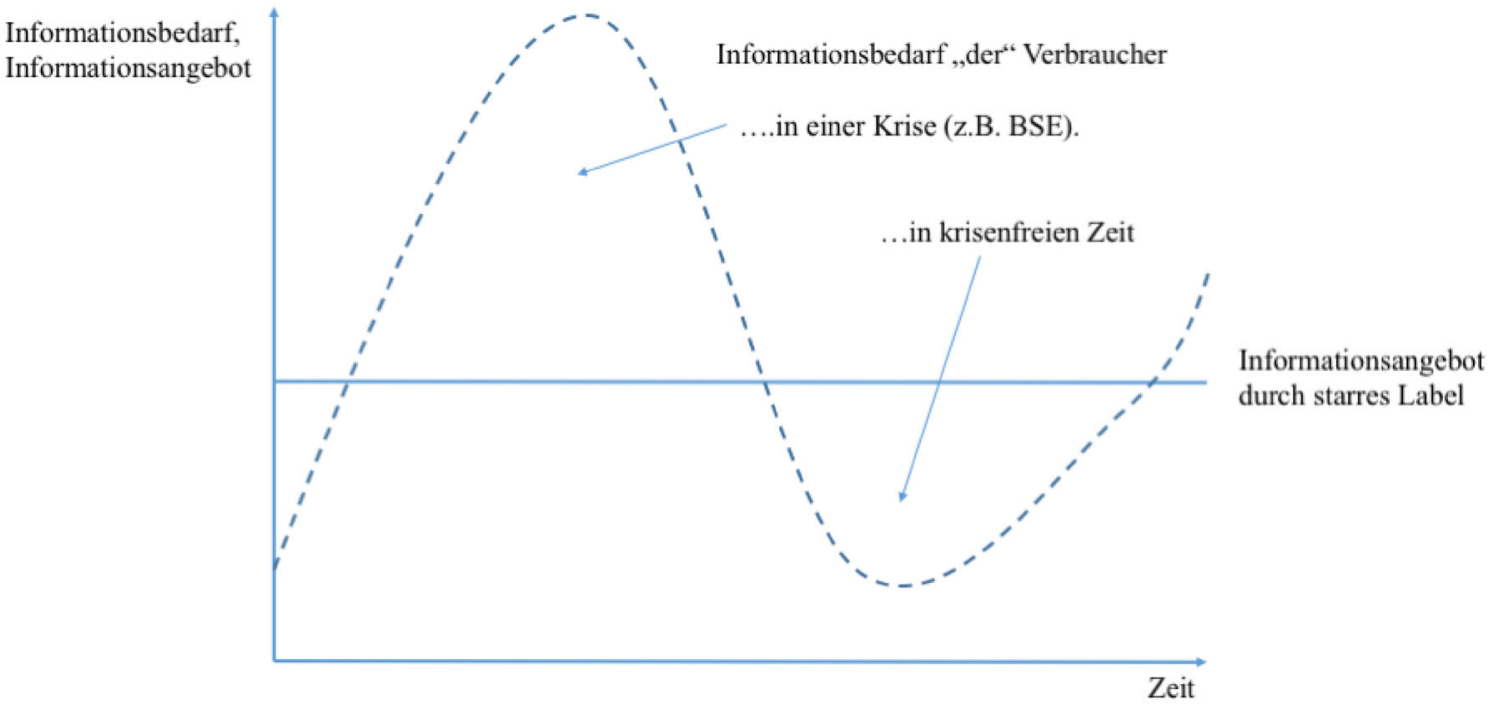

Abb. 1 Informationsangebot und -bedarf im Zeitablauf. Der Informationsbedarf nimmt in Krisenzeiten zu und ist sonst meist gering. Diese Variabilität kann das Informationsangebot durch ein starres Label nicht bedienen

\subsection{Multilayer statt Binarität}

Das oben skizzierte Label-Dilemma hat aus Sicht der Verbraucher Konsequenzen: So wird ein hoher Preis bei unzureichender Information über die „wahren“ Produkteigenschaften oftmals als Barriere gesehen (Boogaard et al. 2006; Larceneux et al. 2012; Napolitano et al. 2010; Padel und Foster 2015). Eine Differenzierung innerhalb der offenbar schwankenden preislichen Grenzen und moralischen Ansichten ist somit kaum möglich: Dem einen Verbraucher ist es zumeist zu teuer, dem anderen ist es zu wenig „bio“. Angesichts dieser Heterogenität wäre es zweckmäßig, den Verbrauchern die Möglichkeit zu eröffnen, nach individuellem Involvement diejenige Produktinformation zu beziehen, welche die informierte Kaufentscheidung nach den eigenen, ggf. zeitlich instabilen, Präferenzen ermöglichen kann. Diese Möglichkeit ließe sich durch die Integration eines sogenannten multi-layer Labelsystems eröffnen (de Jonge et al. 2015; Eberle et al. 2011; Weinrich und Spiller 2016). In diesem System geht es nicht nur um die binäre Unterscheidung zwischen gelabelten und ungelabelten Produkten, sondern es wird innerhalb der Labelstruktur in weitere Stufen (Layer) unterschieden. Durch die Einführung von Differenzierungsebenen können somit psychologische Effekte wie zum Beispiel Kompromiss- oder Anziehungseffekte entstehen, welche den Verbrauchern erlauben, nach ihrem Involvement innerhalb ihrer Preisgrenzen zu entschieden. Erste Implementierungen eines noch recht einfachen angebotsseitig induzierten multi-layer Label zeigen sich in den Niederlanden (,,Beter Leven“) bzw. in Dänemark („Bedre Dyrevelfærd“). Empirische Studien bestätigen, dass die so erreichte Ausdifferenzierung der Labelstruktur zu einem höheren Marktanteil von Tierwohlprodukten führt, die Heterogenität und individuellen Bedürfnisse der Verbraucher besser abgedeckt und auch zeitlich schwankende Zahlungsbereitschaften ,abgegriffen“ werden können (de Jonge et al. 2015; Weinrich und Spiller 2016).

Problematisch ist jedoch, dass nach wie vor eine Vielzahl an Information (Tierwohlhaltung, Fairtrade, Gentechnik, Inhaltsstoffe, u.v.m.) auf den Verpackungen angeboten wird, die in den allermeisten Fällen, nicht benötigt wird. So sind einzelne Informationen (z.B. Laktose-, Gluten- oder Nussanteil) nur für spezielle Käufergruppen relevant oder werden nur nach besonderen Vorkommnissen oder moralischen Krisen durch den Verbraucher aktiv nachgefragt. Die damit verbundene Logik ist informationslogistisch ineffizient und kann zudem zu der bereits erwähnten Verwirrung und Überforderung der Verbraucher am PoS führen. Die in Abbildung 1 skizzierte Problematik wäre somit allenfalls teilweise behoben.

Eine Lösung dieser Problematik könnte darin bestehen, den Informationsfluss nach einem anderen Prinzip zu organisieren und den multi-layer Ansatz um eine vertikale, nachfrageorientierte und damit zeitlich flexible Perspektive zu erweitern (Eberle et al. 2011). Im Ergebnis würde der Informationsfluss somit 
nicht nach einem generellen, zeitlich unflexiblen „Push-Ansatz“ organisiert werden, wie es bei einem klassischen Labelansatz der Fall ist, sondern vielmehr nach einem „Pull-Prinzip“, welches nicht nur zeitlichflexibel wäre, sondern auch den verschiedenen verbrauchertypenspezifischen Informationsbedarfen/typen, die sich situativ ändern können, entsprechen
Informationsbedarf dieser Verbrauchertypen informationslogistisch effizient befriedigt werden und integriert in einen klar vorgegebenen politischen Rahmen könnte eine entsprechende Labelflut möglicherweise verhindert werden (Eberle et al. 2011).

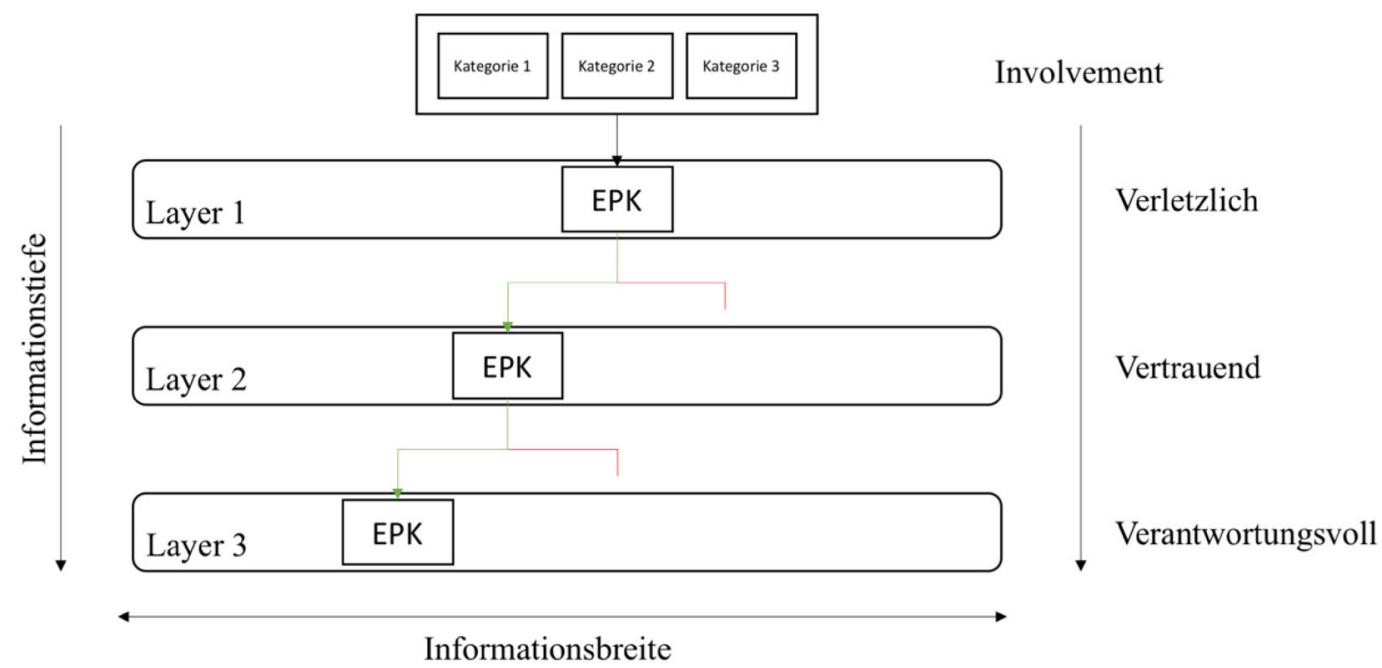

Abb. 2 Konzeption eines vertikalen und horizontal differenzierten Multi-layer Informationssystems. Durch eine Integration von ereignisorientierten Prozessketten (EPK) kann der Verbraucher Informationen, welche der gewünschten Informationstiefe und breite entsprechen, aktiv anfragen

würde (Micklitz et al. 2010; Wobker et al. 2012). Bei dieser Lösung könnten sich Verbraucher bspw. anhand von aufeinander aufbauenden Fragen, die der Logik sogenannter „ereignisorientierter Prozessketten“ (EPK) entspricht (analog zur Organisation von betrieblichen Informationsflüssen im Rahmen von Managementinformationssystemen (z.B. SAP R/3)), die Informationen in der Informationstiefe beschaffen, welche ihrem (situativen) Involvement bzw. Typ entsprechen. Durch bereits bekannte Technologien, wie einen QR-Code über eine Smartphone-App oder einem im Markt installierten Informationsterminal, könnten so verantwortungsvolle Verbraucher bspw. bei einer Produktneueinführung im Bereich der „Fleischware“ das Produkt einscannen. Sie würden dann allgemeine Informationen zum Produkt erhalten, welche zum Beispiel das Produkt zunächst nach einer einfachen multi-layer Labellogik kennzeichnen. Anschließend und anhand der integrierten EPK könnten diese Verbraucher individuell detailliertere Informationen zum Produkt auf den verschiedenen Layerebenen erlangen (Abb. 2). Durch diese Konzeption würde der breitere und tiefere

\subsection{Zur Konzeption eines} Verbraucherinformationssystems im Rahmen der Nutztierhaltung

Ein dieser Konzeption entsprechender Ansatz, der eine Vielzahl an bereits vorhandenen Verbraucherinformationen integrieren könnte und darüber hinaus eine bedarfsgerechte, situative Informationsbeschaffung ermöglichen würde, bestünde in der Entwicklung eines öffentlich verfügbaren Verbraucherinformationssystems (VIS) am PoS. Dieses für die Nutztierhaltung durchaus innovative System soll im Folgenden kurz skizziert werden.

Ein Informationssystem ist ein Ansatz, welcher in der Betriebswirtschaft ursprünglich Informationsnachfragen effizient und effektiv in ein System integrieren sollte (Becker und Schütte 2004; Schütte 2011). Als VIS kann man analog hierzu ein System bezeichnen, welches den Verbrauchern ermöglicht, durch die Nutzung von Informationstechnologien und dementsprechend informierte Kaufentscheidungen die Wertschöpfungskette nach dem Pull- 
Prinzip weiterzuentwickeln und mitzugestalten (Tuunanen et al. 2010).

Anders als bei Informationssystemen bspw. im beruflichen Kontext, wo Nutzer auf das System für ihre Arbeitstätigkeit angewiesen sind und vor allem Effektivität und Effizienz wichtige Parameter darstellen, sollte bei dem individuellen Gebrauch durch Verbraucher eine Balance zwischen Nützlichkeit und Benutzerfreundlichkeit gefunden werden. Denn nur wenn die Verbraucher einen utilitarischen und hedonischen Nutzen erfahren, wird sich ein solches System dauerhaft etablieren können (Tuunanen et al. 2010). Ähnliche Anwendung zeigen sich bereits für Obst und Gemüse (Max Rubner-Institut 2015) und im deutschen Bäckerhandwerk (baeckerhandwerk.de). Mit Hilfe eines Informationsterminals können sich Kunden dort über die angebotenen Waren informieren und individuelle Produktinformationen abrufen. Dadurch wird kein unverständliches Etikettierungssystem benötigt und fachkundige Beratungsgespräche werden durch eine weitere Informationsquelle ergänzt. Welche Ansätze und Herausforderungen ein solches VIS aus theoretisch-konzeptioneller Sicht integrieren müsste und welche Treiber der Verbraucher den Gebrauch ermöglichen, wurde bereits in einem ersten Rahmenkonzept zusammengefasst, welches insbesondere den folgenden Aspekten Rechnung trägt (vgl. Tuunanen et al. 2010). Ein VIS sollte die Verbraucher individuell nach situativer und persönlicher Relevanz und Involvement über die Produkte informieren, sodass diese selbst bestimmen, welche Informationen sie wann erhalten wollen. Dies könnte zum Beispiel anhand von QR-Codes in Kombination mit Smartphone-Apps oder Informationsterminals im Markt realisiert werden.

Das VIS sollte eine Schnittstelle zu sozialen Netzwerken beinhalten. Dort sollte eine unabhängige Moderation ergänzt durch Expertenmeinungen von Landwirten, Händlern und Wissenschaftlern stattfinden. Im Bereich der Nutztierhaltung könnten bspw. verifizierte Nutzer (Verbraucher) aktuelle, für sie relevante Themen (z.B. Medienberichte und Warnhinweise) untereinander und mit unabhängigen Experten diskutieren und Erfahrungen (z.B. Meinungen, Kochideen und Angebote) austauschen. Dadurch wird eine Identitätskonstruktion erzielt, welche den Nutzer an den Service bindet und auch ein Crowdsourcing ermöglicht (vgl. Enkel in Kenning/Lamla 2017). Zudem sollte das VIS den Anwendungskontext (z.B. am PoS) berücksichtigen, da dieser einen Einfluss auf das Nutzungsverhalten haben wird. Verbraucher sind in diesem System ein wichtiges, zentrales Element und können die Gestaltung und Nützlichkeit des Informationssystems durch ihren Gebrauch entscheidend beeinflussen (Tuunanen et al. 2010). Der Gestaltungsprozess des VIS ist entsprechend voraussetzungsvoll: Zum einen sind der Zeitpunkt und die Art der Teilnahme an der Gestaltung des Service durch die Verbraucher festzulegen, sodass die Ziele und Ansprüche der Verbraucher an das VIS den gewünschten Nutzen erzeugen. Zum anderen müssen die Informationen, welche in dem VIS verwendet werden, effektiv und effizient aggregiert und integriert werden (vgl. Oehler und Kenning 2013). Denkbar wäre es, dass bereits vorhandene Systeme kombiniert werden und Informationen aus vertrauensvollen und unabhängigen Quellen integriert werden. Kompatible SAPSysteme, welche bereits vom Handel genutzt werden, könnten im VIS eine Schnittstelle bilden und so Verbrauchern Informationen zum Beispiel zur Herkunft der jeweiligen Produkte bereitstellen. So könnten bspw. handelsbezogene Daten aus den Warenwirtschaftssystemen freigegeben werden und mit weiteren Daten (z.B. aus dem Bundesinformationszentrum Landwirtschaft, BZL) im VIS zu einem multilayer Informationsansatz aufbereitet und verknüpft werden (Abb. 3).

Um den Handel zu motivieren, die jeweiligen Systeme zu öffnen und zu pflegen wäre es denkbar, die entsprechenden Investitionen zu fördern. Das nötige Vertrauen in das VIS könnte durch die Unabhängigkeit und ein glaubwürdiges Monitoring der Inhalte gewährleistet werden. Die verwendeten Daten sollten von öffentlichen Institutionen wie z.B. der Bundesanstalt für Landwirtschaft und Ernährung (BLE) verwaltet werden, wobei es wichtig ist, dass Informationsstandards vereinheitlicht werden und interne Qualitätskriterien, welche sich zurzeit u.a. durch private Bio-Label äußern, sichtbar und transparent von unabhängigen Informationen getrennt werden (Verbraucherkommission Bayern 2012). Angesichts dessen böte sich insgesamt ein modularer, integrativer Aufbau an (Abb. 3). 


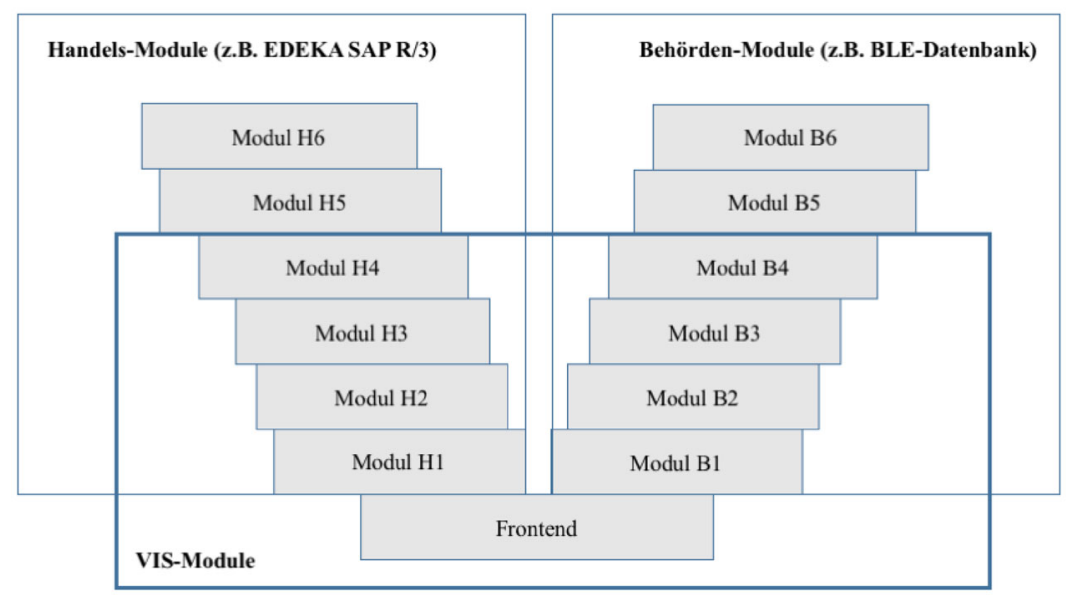

Abb. 3 Datengewinnung und -verwaltung in einem VIS. Informationen und Daten, die in das VIS mit einfließen sollen möglichst kompatibel mit vorhandenen Warenwirtschaftssystem im Handel sein

\section{Ausblick}

Labels bieten mitunter die einfachste Möglichkeit, den Verbraucher am PoS zu informieren. Sie stoßen jedoch oftmals an ihre Grenzen. Aufbauend auf qualitativen und quantitativen Studien kann man rasch erkennen, dass dieser starre Ansatz der Verbraucherinformation möglicherweise durch differenziertere, horizontal und vertikal organisierte Alternativansätze zu optimieren wäre. Sinnvoller wäre es, einen systemischen Ansatz in der Form eines VIS zu verfolgen. Durch ein solches System wäre es möglich, die Informationsbedarfe der Verbraucher flexibel und informationslogistisch optimal zu bedienen.

Die folgenden Ausführungen sind zum Teil das Ergebnis einer umfassenden Diskussion der Autoren mit verschiedenen verbraucherpolitisch aktiven Wissenschaftlern, denen wir zu Dank verpflichtet sind. Er gilt allen Projektpartnern des Verbundprojektes „SocialLab - Nutztierhaltung im Spiegel der Gesellschaft“, insbesondere den Kollegen des Thünen-Instituts für Marktanalyse (Dr. Inken Christoph-Schulz), der Technischen Universität München (Prof. Dr. Jutta Roosen) und der Georg-August-Universität Göttingen (Prof. Dr. Achim Spiller).

\section{Literatur}

Andersen LM (2011) Animal welfare and eggs - cheap talk or money on the counter? Agric Econ Soc 62:565-584

Becker J, Schütte R (2004) Handelsinformationssysteme. Redline Wirtschaft, Frankfurt am Main
Boogaard BK, Oosting SJ, Bock BB (2006) Elements of societal perception of farm animal welfare: a quantitative study in the Netherlands. Livestock Sci 104:13-22

de Jonge J, van der Lans IA, van Trijp HCM (2015) Different shades of grey: compromise products to encourage animal friendly consumption. Food Qual Prefer 45:87-99

Eberle U, Spiller A, Becker T, Heißenhuber A, Leonhäuser IU, Sundrum A (2011) Politikstrategie Food Labelling. http://www.bmelv.de/Shared Docs/Downloads/Ministerium/Beiraete/Verbraucherpolitik/2011_10_PolitikstrategieFoodLabelling.pdf. Abgerufen 10. Oktober 2017

Enkel E (2017) Die Rolle des Konsumenten im Kontext der Open Innovation. In: Kenning P, Lamla J (Hrsg.) Entgrenzungen des Konsums. Springer, Wiesbaden

Franz A, von Meyer M, Spiller A (2010) Prospects for a european animal welfare label from the german perspective: supply chain barriers. Int J Food Syst Dyn 4:318-329

Grunert K (2002) Current issues in the understanding of consumer food choice. Trends Food Sci Technol 13:275-285

Harper G, Henson S (2001) Consumer concerns about animal welfare and the impact on food choice the final report. The University of Reading. Unted Kingdom. EU FAIR CT98-3678

Janssen M, Hamm U (2012) Product labelling in the market for organic food: consumer preferences and willingness-to-pay for different organic certification logos. Food Qual Preference 25:9-22

Kenning P, Oehler A, Reisch L, Grugel C (2017) Verbraucherwissenschaften - Rahmenbedingungen, 
Forschungsfelder und Institutionen. Springer, Wiesbaden

Krüger RA, Casey MA (2014) Focus groups: a practical guide for applied research. Sage, Los Angeles

Larceneux F, Benoit-Moreau F, Renaudin V (2012) Why might organic labels fail to influence consumer choices? Marginal labelling and brand equity effects. J Consumer Policy 35:85-104

Max Rubner-Institut (2015) Verbundprojekt: Verbraucherinformationssystem zur Nutzung am Point of Sale (POS) zum Shelflife und Produkteigenschaften für Obst und Gemüse am Beispiel der Kiwi

Micklitz HW, Oehler A, Piorkowsky MB, Reisch LA, Strünck C (2010) Der vertrauende, der verletzliche oder der verantwortungsvolle Verbraucher? Plädoyer für eine differenzierte Strategie in der Verbraucherpolitik Stellungnahme des Wissenschaftlichen Beirats Verbraucher- und Ernährungspolitik beim BMELV

Napolitano F, Braghieri A, Piasentier E, Favotto S, Naspetti S, Zanoli R (2010) Effect of information about organic production on beef liking and consumer willingness to pay. Food Qual Prefer 21:207-212

Oehler A, Kenning P (2013) Evidenzbasierung ermöglichen! Auf dem Weg zu einer realitätsnahen und empirisch fundierten Verbraucherpolitik - Stellungnahme des wissenschaftlichen Beirats Verbraucher- und Ernährungspolitik beim BMELV

Olaizola A, Corcoran K (2003) Labelling information demanded by European consumers and relationships with purchasing motives. Qual Safety Meat 65:1095-1106

Padel S, Foster C (2015) Exploring the gap between attitudes and behaviour Understanding why consumers buy or do not buy organic food. Br Food J 107:606-625

Padilla C, Villalobos P, Spiller A, Henry G (2007) Consumer preference and willingness to pay for an officially certified quality label: implications for traditional food producers. Agricultura Técnica 67:300-308

Reisch LA (2003) Transparenz auf Nahrungsmittelmarkten: Theoretische Begründung und verbraucherpolitische Praxis. Hauswirtschaft und Wissenschaft - Europäische Zeitschrift für Haushaltsökonomie, Haushaltstechnik und Sozialmanagement

Roosen J, Lusk JL, Fox JA (2003) Consumer demand for and attitudes toward alternative beef labeling strategies in France, Germany, and the UK. Agribusiness 19:77-90

Schütte R (2011) Modellierung von Handelsinformationssystemen. Unveröffentlichte Habilitationsschrift, Westfälische Wilhelms-Universität Münster

Tuunanen T, Myers MD, Cassab H (2010) A conceptual framework for consumer information. Pacific Asia J Assoc Inf Syst 2:47-66

Verbeke W (2005) Agriculture and the food industry in the information age. Eur Rev Agric Econ32:347-368

Verbeke W (2008) Impact of communication on consumers' food choices. Proc Nutr Soc 67:281-288

Verbeke W, Ward RW (2006) Consumer interest in information cues denoting quality, traceability and origin: an application of ordered probit models to beef labels. Food Qual Prefer 17:453-467

Verbraucherkommission Baden-Württemberg (2011) Vom Labelmissbrauch zu Vertrauenslabeln

Verbraucherkommission Bayern (2012) Empfehlungen zum Thema Lebensmittelkennzeichnung. 1-19

vzbv (2017, 29 September) Staatliches Tierwohl-Label muss kommen [Pressemeldung]. Abgerufen von https://www.vzbv.de/pressemitteilung/staatlichestierwohl-label-muss-kommen

Weinrich R, Spiller A (2016) Developing food labelling strategies: multi-level labelling. J Clean Product 137:1138-1148

Wobker I, Lehmann-Waffenschmidt M, Kenning P, Gigerenzer G (2012) What do people know about the economy? A test of minimal economic knowledge in Germany. Dresden Discussion Paper in Economics 3:1-48 


\section{Der Einfluss von Nachrichtentexten über die Tierhaltung auf das soziale Vertrauen in Landwirte}

\section{Sabine Groß und Jutta Roosen}

Lehrstuhl für Marketing und Konsumforschung, TUM School of Management, Technische Universität München, Alte Akademie 16, 85354 Weihenstephan, sabine.gross@tum.de

\section{Zusammenfassung}

Vertrauen kommt eine wichtige Bedeutung in wirtschaftlichen Abläufen zu. Besonders in Verbindung mit landwirtschaftlichen Produkten ist Vertrauen nötig, da viele Attribute nicht vom Verbraucher überprüft werden können und dieser damit auf die Aussagen von Produzenten und Verbraucherorganisationen angewiesen ist. Kritisch ist weiterhin, dass Vertrauen asymmetrische Eigenschaften aufweist und dadurch leichter zerstört als gebildet werden kann. In einer Untersuchung mit 1600 Teilnehmern werden die Dynamiken der Entstehung und Zerstörung von Vertrauen beleuchtet. Mittels verschiedener Nachrichtentexte wird geprüft, wie verschiedene Absender und Tendenzen einer Nachricht das soziale Vertrauen von Verbrauchern beeinflussen können. Ergebnisse zeigen, dass die Effekte negativer Nachrichten stärker sind als die positiver Nachrichten. Weiterhin ist die Wirkung einer Nachricht vom Absender abhängig. Für die gesamte Stichprobe steigert eine positive Nachricht seitens der Politik das Vertrauen und eine negative Nachricht einer Verbraucherorganisation senkt das Vertrauen. Allgemein wirken die gezeigten Nachrichtentexte negativ auf jene Personen, die zuvor ein hohes Vertrauen besaßen.

\section{Einführung}

Vertrauen kommt eine besondere Bedeutung für jene Bereiche zu, in denen wenig Wissen vorherrscht (Siegrist und Cvetkovich 2000; Grabner-Kräuter und Kaluscha 2003) oder auch in riskanten Situationen (Mayer et al. 1995). Dies trifft auch für typische Vertrauensattribute beim Kauf von Lebensmitteln, wie der Produktionsweise, zu (Kenning und Wobker 2012). Hier sind Verbraucher angewiesen auf Informationen, die sie von Produzenten oder Händlern, aber auch Verbraucherorganisationen erhalten.

Vertrauen wird auch verwendet, um eine Verbrauchertypologie zu erstellen. Entsprechend ihrem Risikoempfinden und der Art und Weise ihrer Informationsgewinnung unterscheiden Micklitz u. a. (2010) drei verschiedene Idealtypen von Verbrauchern: „verletzliche“, „verantwortungsvolle“ und „vertrauende“ Verbraucher. Der „vertrauende“ Verbraucher greift aufgrund von Zeit- und Interessensmangel auf die Informationen glaubwürdiger Institutionen zurück, wohingegen der „,verletzliche“ Verbraucher eher überfordert ist und der ,verantwortungsvolle“ Verbraucher sich möglichst vollständig informiert.

Bezüglich verschiedener Quellen zur Informationsgewinnung zeigen Untersuchungen einerseits, dass verschiedene Quellen als unterschiedlich vertrauenswürdig wahrgenommen werden (Chryssochoidis et al. 2009). Zusätzlich kann das Framing unterschiedlicher Quellen einen Einfluss darauf haben, dass Nachrichten als wahr oder falsch interpretiert werden (Deppe et al. 2005). Rosati und Saba (2004) zeigen, dass bei der Bewertung der Informationsquelle im Zusammenhang mit Lebensmittelrisiken den Verbraucherorganisationen ein höheres Vertrauen entgegen gebracht wird als Regierungen oder Produzentenorganisationen. Auch bei Gütesiegeln variiert das Vertrauen in Abhängigkeit davon, ob das Siegel von Regierungs- oder Nichtregierungsorganisationen bzw. der Industrie vergeben wird (McKendree et al. 2013). Ein hohes Vertrauen von Verbrauchern in die Hersteller von Produkten ist gerade für Produzenten wichtig, da Vertrauen die Zahlungsbereitschaft steigern und den Einfluss von negativen Nachrichten puffern kann (Roosen et al. 2015).

Besonders die Informationen, die durch Medien wie Internet, Fernsehen oder Rundfunk verbreitet werden, können von Produzenten kaum gesteuert werden. Daher ist es wichtig, den Einfluss von verschiedenen Informationen auf das Vertrauen zu kennen. Generell ist der Einfluss von positiven und negativen Nachrichten ungleich. Negative Nachrichten oder Ereignisse bleiben länger im Gedächtnis, haben einen größeren Effekt und sind glaubwürdiger (Slovic 1993; Cvetkovich et al. 2002). Vertrauen besitzt asymmetrische Eigenschaften. Es ist möglich, Vertrauen relativ leicht zu zerstören, jedoch ist der Aufbau von Vertrauen ein langwieriger Prozess (Slovic 1993). Daher ist es wichtig die Dynamiken der Entstehung und Zerstörung von Vertrauen, gerade im Bereich der Landwirtschaft und landwirtschaftlicher Produktionsarten, zu ergründen. Dieses Thema ist von besonderer Bedeutung, da die Lebensmittelproduktion von verschiedenen Medien mit unterschiedlichen Tendenzen diskutiert wird. Hier trifft der Gegensatz aufeinander, dass Studien einerseits von einem hohen Vertrauen in den Beruf des Landwirts berichten (Bürkl et al. 2014). Andererseits fehlt für viele Landwirte jedoch die 
wahrgenommene gesellschaftliche Akzeptanz ihres Tuns.

Obwohl Vertrauen Gegenstand vieler Forschungsbereiche ist, fehlt es an einer allgemeingültigen Definition. Häufig wird die Definition von Rousseau et al. (1998) vorgebracht, dass beim Vertrauen die eigene Verwundbarkeit in Kauf genommen wird und gleichzeitig beim Gegenüber positive Absichten unterstellt werden. Es wird in der Literatur zwischen personellen und institutionellen Vertrauen unterschieden, um zu beschreiben, ob es sich bei dem zu Vertrauenden um eine Person oder eine Institution handelt (Poortinga und Pidgeon 2003; Rampl et al. 2012). Hier wird das Konstrukt des „sozialen Vertrauens" verwendet, das nach Earle und Cvetkovich (1995) beschreibt, dass die Verantwortung für eine bestimmte Aufgabe weitergegeben wird. Earle und Cvetkovich (1999) geben an, dass es sich beim sozialen Vertrauen im Bereich der Risikokommunikation um nicht-personelles Vertrauen handelt, bei dem man die zu vertrauende Person oder Institution nicht kennt. In der vorliegenden Studie soll daher der Einfluss von verschiedenen Nachrichten auf das Vertrauen in Landwirte untersucht werden. Hierzu wurde eine Online-Befragung mit 1600 Teilnehmern durchgeführt. Die Teilnehmer wurden einem von vier Informationsszenarien zufällig zugeordnet und ihr soziales Vertrauen in Landwirte vor und nach der Lektüre eines Nachrichtentextes gemessen. Die Analyse wurde für die Teilstichproben getrennt nach Geschlechtern (männlich und weiblich) und ursprünglichem Vertrauensgrad (hohes und niedriges Vertrauen bei der ersten Messung) durchgeführt. In verschiedenen Studien hat sich gezeigt, dass Frauen weniger Vertrauen als Männer haben (z.B. Alesina und La Ferrara 2002) und sich Nachrichten stärker bei skeptischen Personen auswirken (Poortinga und Pidgeon 2004).

\section{Material und Methoden}

Im Herbst 2016 wurde eine Online-Umfrage mit 1600 Teilnehmern durchgeführt um Aufschluss zur Dynamik der Entstehung und Zerstörung von Vertrauen zu erhalten. Die Stichprobe war für die in Deutschland lebende Bevölkerung bezüglich der Merkmale Alter, Geschlecht, Bildung, Einkommen und Beschäftigungsstatus repräsentativ. Der Fragebogen bestand aus 2 Teilen. Zunächst wurde das Konstrukt „soziales Vertrauen“ nach Earle und Cvetkovich (1995) gemessen. Hierfür gaben die Befragten den Grad ihrer Zustimmung zu 4 Aussagen über Landwirte und Nutztierhaltung auf einer Skala von 1 (Stimme ganz und gar nicht zu) bis 6 (Stimme voll und ganz zu) an. Eine explorative Hauptkomponenten Faktoranalyse bestätigte das Konstrukt „soziales Vertrauen“ mit 3 Aussagen und einem Cronbach's $\alpha$ von 0,875.

Im zweiten Schritt wurden die Teilnehmer zufällig einem von 4 Informationsszenarien zugeordnet. Diese Szenarien beinhalten einen Nachrichtentext von verschiedenen Absendern (Regierung oder Verbraucherverband) und Tendenzen (positiv oder negativ) und sind der Tabelle $1 \mathrm{zu}$ entnehmen. Die Texte wurden für die Befragung auf Basis von Zeitungsartikeln, Pressemitteilungen und Webseiten konzipiert. Die Kategorisierung von Absendern und Tendenzen wurde den Befragten nicht explizit genannt. Die Tendenzen und Absender wurden vorab in einer Vorstudie mit 60 Teilnehmern überprüft, wobei sich die Identifikation der Absender für die Probanden teilweise als schwierig gestaltete. 
Tab. 1 Übersicht der Nachrichtentexte

\section{Politik positiv \\ Deutschland als Vorreiter in Sachen Tierschutz \\ Deutschland nimmt beim Tierschutz europaweit eine Führungsrolle ein. Die in der deutschen Landwirtschaft eingesetzten, modernen Techniken der Tierhaltung ermöglichen es, Nutztiere so artgerecht wie noch nie zu halten. So fördern beispielsweise ideal abgestimmte Futterrationen und helle Laufställe das Tierwohl. Auch die Haltungsformen haben sich in den vergangenen Jahren grundlegend gewandelt und verbessert. Bereits 86 \% aller Legehennen in Deutschland leben in Boden-, Freiland- und Öko-Haltung. In anderen EU-Ländern stammen noch deutlich mehr Legehennen aus ausgestalteter Käfighaltung \\ Die Bundesregierung will auch in Zukunft Vorreiter in Sachen Tierschutz bleiben und arbeitet gemeinsam mit der Landwirtschaft an neuen Wegen zur weiteren Verbesserung der Tierhaltung}

\section{Politik negativ}

Die Haltung zum Tierschutz muss sich ändern

Christian Schmidt, Bundesminister für Ernährung und Landwirtschaft, möchte, dass es den Tieren am Ende seiner Legislaturperiode besser geht als heute. Seit 2002 steht der Tierschutz als Staatsziel im Grundgesetz. „Es wird Zeit, dass wir ein gemeinsames Verständnis vereinbaren, was dies beispielsweise für die Nutztierhaltung konkret bedeutet", sagt Schmidt. Er möchte den Tierschutz weiter stärken, sowie gesetzgeberisch handeln, wo es notwendig ist. Dabei darf es nicht bei schönen Worten bleiben, wie es bisher leider oft der Fall ist. Die Politik will bewertbare und klare Indikatoren entwickeln, die Ziele für die Landwirtschaft vorgeben und auch den Erfolg der freiwilligen Initiativen messbar machen

“Jeder muss an seinem Platz Verantwortung übernehmen - der Staat durch Rahmenbedingungen, die das Wohlbefinden von Tieren fördern, die Landwirte, die es in die Tat umsetzen, und wir als Verbraucher, die an der Ladentheke mitentscheiden", so Schmidt

\section{Verbraucherorganisation positiv}

Vorstand der Verbraucherzentrale betont Fortschritte in der Tierhaltung

Moderne Landwirtschaft, Tierhaltung in großen Beständen und industrielle Verarbeitung von Tieren ist "nichts an sich Schlechtes“. Das betonte der Vorstand des Verbraucherzentrale Bundesverbandes (vzbv) vergangene Woche am Rande des Verbraucherpolitischen Forums seines Verbandes in Berlin. Die

Landwirtschaft in Deutschland sei „Gott sei Dank nicht mehr auf dem Stand von 1800“. Den Nutztieren gehe es heute wesentlich besser als früher. Der Vorstand plädierte dafür, in der Kommunikation gegenüber den Verbrauchern ein realistisches Bild der heutigen Landwirtschaft zu vermitteln. Es sei für den Verbraucher irreführend, wenn vor allem bei der Tierhaltung ein „überholtes Bild der Landwirtschaft, dass es heute nicht mehr gibt“, gezeichnet werde. Das gelte es zu vermeiden, betonte der Verbraucherschützer

\section{Verbraucherorganisation negativ}

Der Mythos der artgerechten Tierhaltung

Die Fleischbranche wirbt zunehmend mit „artgerechter“ Tierhaltung - doch was bedeutet das tatsächlich für das Wohl der Tiere? Alternative Tierhaltungsformen, wie z.B. Freiland oder Bio bei Legehennen, scheinen zwar auf den ersten Blick artgerechter zu sein, garantieren jedoch nicht, dass es den Tieren tatsächlich besser ergeht. Bei der Haltung von landwirtschaftlichen Nutztieren sind Verhaltensstörungen, Krankheiten und Schmerzen an der Tagesordnung. Schweine knabbern sich gegenseitig aus Stress die Schwänze ab, Kühen wird Milch aus kranken Eutern abgepumpt und in Geflügelställen ist Kannibalismus „normal“. Mangelhafte Tierbetreuung und schlechtes Management verursachen eine Vielzahl von vermeidbaren Erkrankungen und Todesfällen bei den Tieren - das gilt für alle Haltungsverfahren, ob bio oder konventionell

Deshalb fordern wir gesetzliche Zielvorgaben für die Tiergesundheit: An die Bedürfnisse der Tiere angepasste Haltungsverfahren müssen zum allgemeinen gesetzlichen Standard werden. Alle Kontrollergebnisse zu Haltungsbedingungen und Gesundheitsdaten müssen veröffentlicht werden. Verstöße müssen konsequent geahndet werden
Anschließend wurde erneut das Vertrauen gemessen, indem die Befragten ihre Zustimmung zu den bereits im ersten Schritt 4 verwendeten Aussagen über Landwirte und Nutztierhaltung angaben. Zunächst werden die deskriptiven Statistiken für die gesamte Stichprobe angegeben. Nach einem globalen Vergleich der Effekte der Nachrichtentexte mittels Mittelwertsvergleich wird im nächsten Schritt die Stichprobe nach Geschlecht aufgespalten. Mittels jeweils einer einfaktoriellen Varianzanalyse für Männer und Frauen wird der Einfluss der Nachrichtentexte auf das Vertrauen überprüft. Für die Gruppeneinteilung nach dem ursprünglichen Vertrauensgrad wird die Stichprobe am Mittelpunkt der Skala geteilt, sodass eine Gruppe „niedriges Vertrauen “ für Werte $<3$ der ersten Abfrage des sozialen Vertrauens und eine Gruppe „hohes Vertrauen“ für Werte $\geq 3$ entsteht. 
Tab. 2 Deskriptive Statistik der Gesamtstichprobe

\begin{tabular}{|c|c|c|c|c|}
\hline Variable & \multicolumn{2}{|l|}{ Definition } & $\begin{array}{l}\text { Mittel- } \\
\text { wert }\end{array}$ & $\begin{array}{l}\text { Standard- } \\
\text { abweichung }\end{array}$ \\
\hline Geschlecht & \multicolumn{2}{|l|}{$1=$ weiblich, $0=$ männlich } & 0,506 & 0,500 \\
\hline Alter & & & 48,658 & 15,610 \\
\hline \multirow[t]{7}{*}{ Bildung } & \multicolumn{2}{|l|}{ In 6 Kategorien } & & \\
\hline & (Noch) kein Schulabschluss & $1,6 \%$ & & \\
\hline & $\begin{array}{l}\text { Hauptschulabschluss } \\
\text { (Volksschulabschluss) }\end{array}$ & $31,7 \%$ & & \\
\hline & $\begin{array}{l}\text { Abschluss der polytechnischen } \\
\text { Oberschule }\end{array}$ & $7,2 \%$ & & \\
\hline & $\begin{array}{l}\text { Realschulabschluss, } \\
\text { Handelsschule (Mittlere Reife) }\end{array}$ & $27,3 \%$ & & \\
\hline & $\begin{array}{l}\text { Fachabitur, Abitur (Allgemeine } \\
\text { oder fachgebundene } \\
\text { Hochschulreife) }\end{array}$ & $15,2 \%$ & & \\
\hline & $\begin{array}{l}\text { Fachhochschulabschluss, } \\
\text { Hochschulabschluss }\end{array}$ & $16,9 \%$ & & \\
\hline Haushaltsgröße & \multicolumn{2}{|l|}{ Personen im Haushalt } & 2,230 & 1,097 \\
\hline \multirow[t]{12}{*}{ Einkommen } & \multicolumn{2}{|c|}{$\begin{array}{l}\text { Gemessen als monatliches Haushalts- } \\
\text { Nettoeinkommen }\end{array}$} & & \\
\hline & Unter 500 Euro & $2,1 \%$ & & \\
\hline & 500 bis 899 Euro & $7,1 \%$ & & \\
\hline & 900 bis 1.299 Euro & $13 \%$ & & \\
\hline & 1.300 bis 1.499 Euro & $8,9 \%$ & & \\
\hline & 1.500 bis 1.699 Euro & $7,2 \%$ & & \\
\hline & 1.700 bis 1.999 Euro & $9,6 \%$ & & \\
\hline & 2.000 bis 2.599 Euro & $16,4 \%$ & & \\
\hline & 2.600 bis 3.199 Euro & $12,3 \%$ & & \\
\hline & 3.200 bis 4.499 Euro & $14,8 \%$ & & \\
\hline & 4.500 bis 5.999 Euro & $6,1 \%$ & & \\
\hline & Mehr als 6.000 Euro & $2,5 \%$ & & \\
\hline \multirow[t]{4}{*}{ Vertrauen } & \multicolumn{2}{|l|}{$\begin{array}{l}\text { Männer vor dem Erhalt einer } \\
\text { Nachricht }\end{array}$} & 3,581 & 1,104 \\
\hline & \multicolumn{2}{|c|}{ Frauen vor dem Erhalt einer Nachricht } & 3,457 & 1,178 \\
\hline & \multicolumn{2}{|c|}{$\begin{array}{l}\text { Männer nach dem Erhalt einer } \\
\text { Nachricht }\end{array}$} & 3,561 & 1,090 \\
\hline & \multicolumn{2}{|l|}{$\begin{array}{l}\text { Frauen nach dem Erhalt einer } \\
\text { Nachricht }\end{array}$} & 3,439 & 1,218 \\
\hline Niedriges & \multicolumn{2}{|l|}{$\begin{array}{l}1=\text { wenn Wert des Konstrukts } \\
\text { „soziales }\end{array}$} & 0,244 & \\
\hline $\begin{array}{l}\text { ursprüngliches } \\
\text { Vertrauen }\end{array}$ & \multicolumn{2}{|c|}{$\begin{array}{l}\text { Vertrauen“ vor der Nachricht kleiner } \\
\text { als } 3 \text { ist, } 0=\text { wenn nicht }\end{array}$} & & \\
\hline
\end{tabular}

\section{Ergebnisse}

\subsection{Deskriptive Statistiken}

Die Ergebnisse der deskriptiven Analyse sind in Tabelle 2 dargestellt und geben neben dem Mittelwert auch die Standardabweichung an. Die Stichprobe war repräsentativ für die deutsche Bevölkerung und weist annähernd eine Gleichverteilung der Geschlechter auf. Im Durchschnitt waren die Befragten 48,7 Jahre alt. Mindestens eine fachgebundene Hochschulreife besaßen $32,1 \%$ der Befragten. Die Haushaltsgröße betrug 2,3 Personen und das monatliche Netto-Haushaltseinkommen lag bei der Mehrheit der Teilnehmer (52,1\%) über 2.000 Euro. Die Einteilung der Stichprobe entsprechend ihres ursprünglichen Vertrauens ergibt, dass etwa $24 \%$ der Befragten ein niedriges soziales Vertrauen zu Beginn der Befragung aufweisen.

\subsection{Globale Effekte der Nachrichtentexte}

Zunächst wird der Gesamteffekt der 4 Nachrichtentexte untersucht. Die Ergebnisse des Mittelwertsvergleichs sind der Tabelle 3 zu entnehmen. Nur die positive Nachricht der Politik und die negative Nachricht einer Verbraucherorganisation konnten signifikante Ergebnisse im Mittelwertsvergleich erzielen und sind entsprechend der Erwartung vertrauenssteigernd bzw. vertrauenssenkend. Der Effekt der negativen Nachricht der Verbraucherorganisation war dabei größer als jener der positiven Nachricht seitens der Politik $(-0,32$ gegenüber $+0,21$ ). Der nächste Schritt stellt die Wirkung der Nachrichtentexte nach Geschlecht und ursprünglichem Vertrauen dar.

Tab. 3 Mittelwertsvergleich der Vertrauensänderung nach Nachrichtentexten

\begin{tabular}{llllll}
\hline & Politik & & & \multicolumn{2}{l}{ Verbraucherorganisation } \\
\cline { 2 - 3 } \cline { 5 - 6 } \cline { 5 - 6 } $\begin{array}{l}\text { Veränderung } \\
\text { Vertrauen }\end{array}$ & $+0,21^{* * *}$ & $-0,04$ & $+0,05$ & $-0,32^{* * *}$ \\
\hline
\end{tabular}

*, ${ }^{* *},{ }^{* * *}$ geben die Ergebnisse eines t-Tests mit einem Signifikanzlevel von 0,05, 0,01, 0,001 an 


\subsection{Effekte der Nachrichtentexte nach Geschlecht und ursprünglichem Vertrauen}

Die Ergebnisse der einfaktoriellen Varianzanalyse sind in Tabelle 4 für die Teilstichprobe Männer und in Tabelle 5 für Frauen dargestellt. Es wurde jeweils zwischen den 4 verschiedenen Nachrichtentexten unterschieden und jeweils nach ursprünglich hohem und niedrigen Vertrauen. Es zeigt sich dabei, dass die individuellen Veränderungen innerhalb einer Gruppe nicht signifikant sind, jedoch zwischen den Gruppen signifikante Unterschiede bestehen. Der F-Test auf unterschiedliche Mittelwerte zwischen den Gruppen ist sowohl für die Teilstichprobe der Männer mit $F(7,782)=12,71$, wie auch für die der Frauen mit $F(7,802)=20,80$ hochsignifikant (p $<0,001)$.

Die Lektüre eines Nachrichtentextes führt bei Männern mit ursprünglich niedrigem Vertrauen grundsätzlich zu einer Vertrauenssteigerung. Den größten Effekt erzielt dabei die positive Nachricht einer Verbraucherorganisation $(+0,340)$. Dahingegen senken alle Nachrichtentexte das Vertrauen von Männern, wenn sie ursprünglich ein hohes Vertrauen besaßen. Hier ist die stärkste Veränderung bei der negativen Nachricht einer Verbraucherorganisation $(-0,333) \mathrm{zu}$ sehen. Insgesamt führen die Nachrichtentexte bei Männern zu einer Vertrauenssenkung. Mittels einem Bonferroni Post-Hoc Test wurden die Unterschiede zwischen den Personen mit ursprünglich niedrigen und hohen Vertrauen überprüft. Mit Ausnahme der negativen Nachricht der Politik $(\mathrm{p}=0,053)$ sind alle Unterschiede in der Wirkung der Nachrichtentexte statistisch signifikant $(\mathrm{p}<0,01)$.

Tab. 4 Mittelwert der Vertrauenseffekte der Informationsszenarien für Männer, aufgeschlüsselt nach ursprünglichem Vertrauen, Standardabweichung in Klammern

\begin{tabular}{lll}
\hline Politik positiv** & Niedriges Vertrauen & $+0,340$ \\
& & $(0,766)$ \\
& & $-0,074$ \\
& Hohes Vertrauen & $(0,631)$ \\
Politik negativ & Niedriges Vertrauen & $+0,299$ \\
& & $(0,625)$ \\
& Hohes Vertrauen & $-0,090$ \\
& & $(0,652)$ \\
Verbraucherorganisation positiv*** & Niedriges Vertrauen & $+0,496$ \\
& & $(0,741)$ \\
& Hohes Vertrauen & $-0,016$ \\
& & $(0,641)$ \\
Verbraucherorganisation negativ*** & Niedriges Vertrauen & $+0,317$ \\
& & $(0,915)$ \\
& Hohes Vertrauen & $-0,333$ \\
Anzahl & & $(0,778)$ \\
Gesamt & & 790 \\
& & $-0,021$ \\
& & $(0,735)$
\end{tabular}

${ }^{* * *},{ }^{* *},{ }^{*}$ geben ein Signifikanzlevel von 0,05, 0,01 und 0,001 an mit einem PostHoc Bonferroni Test
Frauen reagieren ähnlich auf die Lektüre eines Nachrichtentextes wie Männer. Grundsätzlich wirken Nachrichten auf Frauen mit ursprünglich niedrigem Vertrauen vertrauenssteigernd. Hiervon ist jedoch die negative Nachricht einer Verbraucherorganisation ausgenommen, die ebenfalls bei Frauen mit ursprünglich niedrigem Vertrauen das Vertrauen senkt $(-0,175)$. Die stärkste Veränderung bei Frauen mit ursprünglich niedrigem Vertrauen erzielte die positive Nachricht seitens der Politik $(+0,594)$. Nachrichtentexte führen bei Frauen mit ursprünglich hohen Vertrauen zu einer Senkung des Vertrauens. Hier bildet die positive Nachricht seitens der Politik die Ausnahme, die auch für Frauen mit ursprünglich hohem Vertrauen dieses erhöht (+ 0,304). Die größte Veränderung bewirkte bei Frauen mit ursprünglich hohem Vertrauen die negative Nachricht der Verbraucherorganisation (- 0,553). Insgesamt senken die Nachrichtentexte das Vertrauen von Frauen. Der Bonferroni Post-Hoc Test zeigte für die negative Nachricht der Politik $(\mathrm{p}<0,01)$ und die positive Nachricht der Verbraucherorganisation $\quad(\mathrm{p}<0,01) \quad$ signifikante Unterschiede zwischen den Frauen mit ursprünglich niedrigem und hohem Vertrauen.

Tab. 5 Mittelwert der Vertrauenseffekte der Informationsszenarien für Frauen, aufgeschlüsselt nach ursprünglichem Vertrauen, Standardabweichung in Klammern

\begin{tabular}{lll}
\hline Politik positiv & Niedriges Vertrauen & $+0,594$ \\
& & $(1,076)$ \\
& Hohes Vertrauen & $+0,304$ \\
& & $(0,697)$ \\
Politik negativ** & Niedriges Vertrauen & $+0,326$ \\
& & $(0,756)$ \\
& Hohes Vertrauen & $-0,169$ \\
Verbraucherorganisation positiv** & & $(0,707)$ \\
& & $+0,365$ \\
& & $(0,699)$ \\
Verbraucherorganisation negativ & Hohes Vertrauen & $-0,112$ \\
& & $(0,816)$ \\
& Niedriges Vertrauen & $-0,175$ \\
Anzahl & & $(0,732)$ \\
Gesamt & Hohes Vertrauen & $-0,553$ \\
& & $(0,939)$ \\
& & 810 \\
& & $-0,018$ \\
& & $(0,872)$ \\
\hline
\end{tabular}

***, ${ }^{* *},{ }^{*}$ geben ein Signifikanzlevel von 0,05, 0,01 und 0,001 an mit einem PostHoc Bonferroni Test 
Grundsätzlich haben Frauen stärker auf die Lektüre eines Nachrichtentextes reagiert mit Ausnahme der Frauen mit niedrigem ursprünglichem Vertrauen, die eine Nachricht einer Verbraucherorganisation erhalten haben.

\section{Diskussion der Ergebnisse und Fazit}

Unser Experiment bestätigt, dass negative Nachrichten einen größeren Einfluss auf das Vertrauen von Verbrauchen haben als positive Nachrichten. Weiterhin rufen generell die positive Nachricht der Politik und die negative Nachricht einer Verbraucherorganisation signifikante Vertrauensänderungen hervor. Es lässt vermuten, dass die beiden anderen Informationsszenarien nicht zu signifikanten Änderungen geführt haben, da es sich um eine nicht-kongruente Verbindung für Verbraucher von Adressat und Tendenz einer Nachricht gehandelt hat. Eine multivariate Auswertung der Nachrichtentexte unter Einbezug der wahrgenommenen Absender könnte hier weiter Aufschluss geben.

Weiterhin konnten wir zeigen, dass Frauen stärker auf das Gelesene reagiert haben als Männer. Eine Panelregression könnte weiteren Aufschluss über den Einfluss von soziodemografischen Angaben und Heterogenität liefern.

\section{Literatur}

Alesina AF, La Ferrara E (2002) Who trusts others? J Public Econ 85:207-234. https://doi.org/10.1016/ S0047-2727(01)00084-6

Bürkl R, Frank R, Müller B (2014) Trust in Professions 2014. GfK Verein 1-70

Chryssochoidis G, Strada A, Krystallis A (2009) Public trust in institutions and information sources regarding risk management and communication: towards integrating extant knowledge. J Risk Res 12:137-185. 13669870802637000

Cvetkovich G, Siegrist M, Murray R, Tragesser S (2002) New information and social trust: asymmetry and perseverance of attributions about hazard managers. Risk Anal 22:359-367. https://doi.org/10. 1111/0272-4332.00030

Deppe M, Schwindt W, Krämer J, et al. (2005) Evidence for a neural correlate of a framing effect: bias-specific activity in the ventromedial prefrontal cortex during credibility judgments. Brain Res Bull 67:413-421. https://doi.org/10.1016/j. brainresbull.2005.06.017

Earle TC, Cvetkovich G (1999) Social trust and culture in risk managment. In: Social Trust and the Management of Risk

Earle TC, Cvetkovich GT (1995) Social trust: toward a cosmopolitan society, 1. publ. Praeger, Westport

Grabner-Kräuter S, Kaluscha EA (2003) Empirical research in on-line trust: a review and critical assessment. Int J Hum Comput Stud 58:783-812. https://doi.org/10.1016/S1071-5819(03)00043-0

Kenning P, Wobker MAI (2012) Affektive und kognitive Verhaltensstrategien zur Überwindung von Informationsasymmetrien im Konsumgüterhandel - Eine empirische Analyse mit kartellrechtlichen Implikationen. Betriebswirtschaftliche Forsch und Prax 64:626-642

Mayer RC, Davis JH, Schoorman FD (1995) An integrative model of organizational trust. Acad Manage Rev 20:709-734

McKendree M, Wydmar N, Ortega D, Foster K (2013) Consumer preferences fo verified pork practices in the production of ham products. J Agric Resour Econ 38:397-417

Micklitz H-W, Oehler A, Piorkowsky M-B, et al. (2010) Der vertrauende, der verletzliche oder der verantwortungsvolle Verbraucher? Plädoyer für eine differenzierte Strategie in der Verbraucherpolitik

Poortinga W, Pidgeon NF (2003) Exploring the dimensionality of trust in risk regulation. Risk Anal 23:961-972. https://doi.org/10.1111/1539-6924.00373

Poortinga W, Pidgeon NF (2004) Trust, the asymmetry principle, and the role of prior beliefs. Risk Anal 24:1475-1486. https://doi.org/10.1111/j.0272-4332. 2004.00543.x

Rampl LV, Eberhardt T, Schütte R, Kenning P (2012) Consumer trust in food retailers: conceptual framework and empirical evidence. Int J Retail Distrib 
Manag 40:254-272. https://doi.org/10.1108/ 09590551211211765

Roosen J, Bieberstein A, Blanchemanche S, et al (2015) Trust and willingness to pay for nanotechnology food. Food Policy 52:75-83. https://doi.org/10.1016/j. foodpol.2014.12.004

Rosati S, Saba A (2004) The perception of risks associated with food-related hazards and the perceived reliability of sources of information. Int J Food Sci Technol 39:491-500. https://doi.org/10.1111/j.13652621.2004.00808.x
Rousseau DM, Sitkin SB, Burt RS, Camerer C (1998) Not so different after all: a cross-discipline view of trust. Acad Manag Rev 23:393-404. https://doi.org/ 10.5465/AMR.1998.926617

Siegrist M, Cvetkovich G (2000) Perception of hazards: the role of social trust and knowledge. Risk Anal 20:713-720. https://doi.org/10.1111/0272-4332.205064 Slovic P (1993) Perceived risk, trust, and democracy. Risk Anal 13:675-682. https://doi.org/10.1111/j.15396924.1993.tb01329.x 
Weiterentwicklung landwirtschaftlicher Nutztierhaltungsverfahren für mehr Tierwohl aus Sicht von Tierhaltern - Ergebnisse aus moderierten Gruppendiskussionen

\author{
Christiane Wildraut ${ }^{1}$ und Marcus Mergenthaler ${ }^{1}$ \\ ${ }^{1}$ Fachhochschule Südwestfalen, Fachbereich Agrar- \\ wirtschaft, Soest \\ wildraut.christiane@fh-swf.de
}

\section{Einleitung}

Die Perspektive von Landwirten auf Tierwohl und Tiergerechtheit ist seit einigen Jahren Gegenstand zahlreicher Untersuchungen (z.B. Spooner et al. 2014; Van Huik \& Bock 2007). Angestoßen durch gesellschaftlich veränderte Einstellungen gegenüber der Nutztierhaltung (WBA 2015) werden branchenintern zunehmend intensive Diskussionen zur Weiterentwicklung von Tierhaltungsverfahren geführt. Die Verbesserung des Tierwohls besitzt für Landwirte in Deutschland dabei inzwischen einen hohen Stellenwert (Zapf et al. 2015). Sie messen Tierwohl vornehmlich über Gesundheits- und Leistungsparameter (Heise \& Theuvsen 2015) und bewerten aktuelle Verfahren der Tierhaltung als positiver gegenüber früheren Verfahren, auch mit Blick auf das Tierwohl (Te Velde et al. 2002; Vanhonacker et al. 2008). Gesellschaftliche und brancheninterne Diskussionen zur Weiterentwicklung von Tierhaltungsverfahren im Hinblick auf mehr Tierwohl werden derzeit weitgehend nebeneinander geführt (WBA 2015). Besonders innerhalb der konventionellen Landwirtschaft führt die gesellschaftliche Kritik in Bezug auf die Nutztierhaltung zu Befürchtungen, die soziale Akzeptanz und damit die "Licence to produce" zu verlieren (Busch et al. 2013; Te Velde et al. 2002). Von gesellschaftlicher Kritik sind Landwirte persönlich betroffen, da sie diejenigen sind, die unmittelbar Einfluss auf die Haltungsbedingungen und damit auf die Gestaltung der Lebensumwelt der Tiere nehmen (Waiblinger 1996). Durch Investitionsentscheidungen in technische und bauliche Haltungssysteme bestimmen sie langfristig die Weiterentwicklung der Nutztierhaltung.

Ziel des vorliegenden Beitrags ist es, die Einstellungen von Tierhaltern zur Weiterentwicklung der Nutztierhaltung in Deutschland zu erfassen und zu analysieren. Damit soll eine Einschätzung aus der Landwirtschaft zu zukunftsfähigen Konzepten mit mehr Tierwohl für die Rinder-, Schweine- und Geflügelhaltung gewonnen werden. Konkret soll untersucht werden, welche Maßnahmen Landwirte einzelbetrieblich und für die Branche sehen und welche Hemmnisse die Konzeptentwicklung und die Umsetzung neuer Haltungsverfahren möglicherweise beeinträchtigen. Aus den Ergebnissen sollen erste Empfehlungen für Beratung und Politik abgeleitet werden, gesellschaftlich akzeptierte und von Tierhaltern befürwortete Verfahren der Tierhaltung zu unterstützen.

\section{Daten und Methode}

Von September bis Dezember 2016 wurden in verschiedenen Schwerpunktregionen der Tierhaltung insgesamt 6 Gruppendiskussionen mit Tierhaltern (Rind, Schwein und Geflügel) zur Weiterentwicklung landwirtschaftlicher Tierhaltungsverfahren durchgeführt. Die Auswahl der Standorte erfolgte unter Berücksichtigung der Viehdichten für die drei Tiergruppen in verschiedenen Regionen Nord-, Süd-, Ost- und Westdeutschlands (bezogen auf Statistische Ämter des Bundes und der Länder 2011). Die Diskussionen zur Schweinehaltung fanden in Nordrhein-Westfalen und in MecklenburgVorpommern, zur Milchviehhaltung in SchleswigHolstein und Bayern und zur Geflügelhaltung in Niedersachsen und in Sachsen-Anhalt statt (Tab. 1). Die Rekrutierung der Landwirte erfolgte über die Deutsche Landwirtschaftsgesellschaft (DLG), über Arbeitskreise, Verbände und Beratungsorganisationen sowie über die Landwirtschaftskammern bzw. -ämter. Bei der Quotierung wurde darauf geachtet, verschiedene Produktionsstufen und Haltungssysteme sowie beim Geflügel verschiedene Tierarten einzubeziehen. Eine weitere Vorgabe bestand darin, dass an jeder Diskussionsrunde mindestens eine Frau teilnehmen sollte, was bei 5 der 6 Diskussionsrunden erreicht werden konnte. Insgesamt haben sich jeweils zwischen 5 und 8 Landwirten an den Diskussionen beteiligt. 
Tab. 1 Teilnehmer und einbezogene Produktionsstufen in den Gruppendiskussionen

\begin{tabular}{|c|c|c|c|c|c|}
\hline \multirow[t]{2}{*}{ Diskussion } & \multirow[t]{2}{*}{ Region } & \multicolumn{2}{|l|}{ Teilnehmer } & \multicolumn{2}{|l|}{ Betriebe } \\
\hline & & Anzahl gesamt & darunter Frauen & Tierart & Produktionsstufe \\
\hline GD_S 1 & Nordrhein-Westfalen & 8 & 2 & Schwein & Sauenhaltung, Ferkelaufzucht, \\
\hline GD_S 2 & Mecklenburg-Vorpommern & 7 & 1 & & $\begin{array}{l}\text { Schweinemast, } \\
\text { geschlossenes System }\end{array}$ \\
\hline GD_R 1 & Schleswig-Holstein & 5 & 2 & Rind & Milchviehhaltung, Jungviehaufzucht \\
\hline GD_R 2 & Bayern & 6 & 1 & & \\
\hline GD_G 1 & Niedersachsen & 8 & 1 & Huhn & Elterntierhaltung, \\
\hline GD_G 2 & Sachsen-Anhalt & 5 & - & $\begin{array}{l}\text { Pute } \\
\text { Ente }\end{array}$ & $\begin{array}{l}\text { Junghennenaufzucht, Legehennenhaltung, } \\
\text { Hähnchen-, Puten- und Entenmast }\end{array}$ \\
\hline
\end{tabular}

Inhaltlich orientierten sich die moderierten Diskussionen an 6 aus der Literatur und eigenen Vorarbeiten abgeleiteten Aspekten der Nutztierhaltung, auf welche die Tierhalter direkt Einfluss nehmen können:

1. Stallbau

2. Ausgestaltung und Angebote

3. Unversehrtheit und Eingriffe

4. Tiergesundheit

5. Tierbetreuung

6. Management

Jedes Thema wurde mit einem vorgegebenen Zeitfenster von maximal 15 Minuten diskutiert. Die Gruppendiskussionen wurden jeweils von 2 Personen moderiert und als Audioaufnahme aufgezeichnet. Anschließend erfolgte eine vollständige Transkription und der Import der Dokumente als gemeinsames Projekt in das Analyseprogramm MAXQDA. Über ein kombiniert induktiv-deduktiv entwickeltes Kategoriensystem mit Codes und Subcodes wurden einzelne Textstellen markiert und die Texte damit für die weitere dokumentenübergreifende qualitativ-inhaltsanalytische Auswertung strukturiert (angelehnt an Mayring 2002).

\section{Ergebnisse}

Die Ergebnisse der Gruppendiskussionen verdeutlichen, dass Landwirte Tierwohl in erster Linie an Gesundheitsmerkmalen und biologischen Leistungen festmachen: GD_G 1 „Also für mich das größte Tierwohl ist, wenn die Tiere eine gute Futterverwertung haben und eine gute Leistung haben und wenig
Medikamente. Das zeigt mir eigentlich, dass die Tiere gesund sind und sich wohlfühlen. ${ }^{\text {"11 }}$

Die Landwirte betonen das hohe Niveau aktueller Haltungsverfahren für Nutztiere in Deutschland. Das gilt in ihren Augen für alle betrachteten Tierarten. Als Referenz werden vergangene Tierhaltungsverfahren oder solche aus anderen Ländern herangezogen. Die Teilnehmer sehen die bisherigen Entwicklungen im Bereich der Tierhaltung in erster Linie als Anpassungsstrategie an wirtschaftliche Rahmenbedingungen: GD S 1 „Im Grunde sind wir dazu gezwungen worden, wenn wir weiter machen wollen, die Tierhaltung in diese Richtung zu entwickeln in der Vergangenheit. Weil die Wirtschaftlichkeit sonst nicht gegeben ist." Dass diese Entwicklungen gleichzeitig zu einem Mehr an Tierwohl geführt haben, wird von den Landwirten als positiver Nebeneffekt gewertet.

Zur Weiterentwicklung der Tierhaltungsverfahren äußern sich die Landwirte nur vorsichtig. Maßnahmen zur Verbesserung des Tierwohls werden meistens in Bezug gesetzt zu wirtschaftlichen und politischen Rahmenbedingungen. Außerdem werden betriebliche und persönliche Hemmnisse deutlich, sich mit Fragen der Weiterentwicklung auseinanderzusetzen. Überwiegend zeigt sich der Wunsch nach Beibehaltung der aktuellen Verfahren: GD_S 1 „Wenn ich ein solches Problem damit hätte, die Tiere $\mathrm{zu}$ halten, wie wir sie halten, dann würde ich etwas verändern. Es geht hier nicht nur um die Wirtschaftlichkeit. Es ist ja auch ein Beruf, den man gerne ausübt. Und das kann man nur, wenn man mit der Haltungsform, die man ausübt, zurechtkommt. Und wenn wir auf dem Betrieb Dinge sehen, die nicht in Ordnung sind, dann verändern wir sie." Gleichwohl

\footnotetext{
${ }^{11}$ Hierbei handelt es sich um Originalwortlaute aus den Gruppendiskussionen.
} 
sehen und benennen die Landwirte zu allen angesprochenen Themen der Tierhaltung Möglichkeiten für mehr Tierwohl, die teilweise tierartenspezifisch gelten und unterschiedliche Relevanz für Tierarten oder Produktionsstufen haben.

\subsection{Stallbau}

Die Tierhalter sehen verschiedene Ansatzpunkte für mehr Tierwohl beim Stallbau bzw. bei der generellen Wahl des Haltungsverfahrens. Dabei machen sie deutlich, dass diese grundsätzlichen Entscheidungen für die Haltung langfristig angelegt sind: GD_S 1 „Wenn der Stall einmal steht, muss der mindestens 20-30 Jahre so stehen." Sie sprechen sich dafür aus, dass die Wahl des Haltungsverfahrens den Bedürfnissen der Tiere angepasst sein sollte, dazu zählen z.B. optimale Licht- und Luftbedingungen oder Wahlmöglichkeiten verschiedener Aufenthaltsbereiche für die Tiere.

Mehrheitlich wird von den Tierhaltern die Stallhaltung gegenüber der Freiland- oder Auslaufhaltung bevorzugt. Das gilt insbesondere in der Geflügel- und in der Schweinehaltung, wo die Stallhaltung gesundheitliche und seuchenhygienische Vorteile bietet. Zudem gehen sie davon aus, dass in der Stallhaltung weniger Medikamente eingesetzt werden als in der Freilandhaltung. In der Geflügelhaltung wird der Wintergarten für bestimmte Regionen als praktikable Alternative für mehr Tierwohl eingeschätzt: GD_G 1 ,Deswegen werde ich für die Stallhaltung plädieren, durchaus in Erweiterung wie Wintergärten." In der Schweinehaltung wird den Tieren zugesprochen, dass Auslaufmöglichkeiten zum Wohlbefinden der Tiere beitragen: GD_S 1 „Offene Ställe und ob ein Schwein wühlen kann, ist für ein Schwein mit Sicherheit besser." Trotzdem überwiegen derzeit aus Sicht der Tierhalter die Vorteile geschlossener Haltungen. Hier wird befürwortet, Ställe an sich attraktiver zu konzipieren, etwa an Beispielen orientiert, die in Nachbarländern bereits umgesetzt werden: GD_S 2 ,...moderne große Ställe ... sehr lichtdurchflutet, im Sauenbereich zumindest mit hohen Gebäuden..., technische Entwürfe, die sehen gar nicht aus wie ein Schweinestall.“

Anders sieht es in der Milchviehhaltung aus, wo die Weidehaltung als deutlich positiver für die Tiere wahrgenommen wird. In großen Betrieben mit hohen Tierzahlen ist die Laufhofhaltung ein Kompromiss. Insgesamt wird deutlich, dass der Blick von außen auf den jeweiligen Betrieb mit Anregungen für neue Haltungskonzepte als sinnvoll eingeschätzt wird, der eigenen Betriebsblindheit entgegenzuwirken: GD_R 2 „Oft ist der Betrieb aber auch ein bisschen blind, und man muss den etwas anstupsen.“

\subsection{Ausgestaltung und Angebote}

Die Ausgestaltung der Haltungsverfahren und die Angebote für die Tiere bieten den Landwirten deutlich mehr und überdies kurzfristige Möglichkeiten, mehr Tierwohl in den Betrieben umzusetzen: GD_S 1 „Da innen drin kann man kurzfristiger auch etwas machen." Angesprochen werden Verbesserungen im Stallklima, die an die Bedürfnisse der Tiere angepasst sind, wie z.B. Kühlung oder Beschattung im Stall bei extremeren Witterungslagen. Die Belegdichten in den Ställen werden kontrovers diskutiert. Obwohl ein größeres Platzangebot als förderlich für das Wohlbefinden der Tiere angesehen wird, wird darin oftmals kein Zusammenhang mit einem verbesserten Gesundheitsstatus der Tiere wahrgenommen: GD_G 2 „Dadurch haben wir schon reduzierte Tierplatzzahlen im Stall und ich kann eigentlich nicht sagen, dass das besser läuft als in anderen Ställen. Da sehe ich überhaupt keinen Zusammenhang."

Als wichtig und zukunftsfähig mit Blick auf mehr Tierwohl werden das Angebot und die Gestaltung verschiedener Funktionsbereiche angesehen. Verbesserungsbedarf wird beispielsweise für die Sauenhaltung gesehen: GD_S 2 „Alternativen zum Kastenstand für die Sauen wären wünschenswert, weil es für die Sau einfach nicht schön ist.“ Bedarf wird auch für die Milchviehhaltung gesehen, wo Standardmaße für Liegeboxen nicht als passend für jedes Einzeltier betrachtet werden. Hier wäre es von Vorteil, flexiblere Systeme zu entwickeln. Mit Blick auf Beschäftigungsmöglichkeiten und Tierkomfort sprechen sich die Landwirte dafür aus, Angebote weiterzuentwickeln, die von den Tieren gut und über einen längeren Zeitraum angenommen werden. Gerade in der Mast sind zusätzliche Maßnahmen gewünscht, die den Tieren Abwechslung bieten. Dazu zählen etwa räumliche Veränderungen in der Haltungsumgebung, z.B. über das zeitlich begrenzte Angebot bestimmter Stallbereiche: GD_G 1 „Das sind Dinge, wenn man es ausprobiert sieht man, dass das den Tieren richtig gut tut."

\subsection{Unversehrtheit und Eingriffe}

Die derzeit üblichen Eingriffe an Nutztieren werden von den Landwirten mit Tierschutzargumenten befürwortet. Eine emotionale Sichtweise der Tierhalter wurde in den Gruppendiskussionen 
ausgeblendet: GD_G 2 „Wenn man damit aufgewachsen ist, dann ist das für uns Gewohnheit.“ Gleichwohl werden auch Unsicherheiten in der Bewertung und die Suche nach Alternativen deutlich. Lösungsmöglichkeiten, auf bestimmte Eingriffe, wie das Kastrieren männlicher Ferkel, das Kupieren von Schwänzen bei Ferkeln, das Entfernen von Hornanlagen bei Kälbern oder das Schnäbelkürzen im Geflügelbereich zu verzichten, sehen die Tierhalter in erster Linie über die Zucht, z.B. auf Hornlosigkeit oder auf ruhige, aggressionsfreie Tiere. Die Immunokastration von Ebern wird eher kritisch gesehen. Die züchterischen Entwicklungen in der Rinderhaltung werden als Schritt in die richtige Richtung gewertet, benötigen aber Zeit: GD_R 1 „Ich finde das Thema muss man auch gar nicht so hochspielen, weil in spätestens zehn Jahren haben wir doch sowieso alle hornlos oder?“ Derzeit behelfen sich einzelne Milchviehhalter damit, männliche Kälber möglichst jung an Mäster weiterzugeben und überlassen dem Mäster den Eingriff: GD_R 2 „Ich finde, das ist ein ganz schwieriges Thema. Bei uns auf dem Betrieb schauen wir, dass wir sie alle mit 3 Wochen aus dem Betrieb rausbekommen." Neben der Zucht sehen die Landwirte auch Möglichkeiten in der Gestaltung der Haltungsumgebung, um einen Verzicht auf Eingriffe am Tier zu vermeiden. Dazu zählt in der Geflügelhaltung eine trockene, lockere Einstreu, die den Tieren Beschäftigung bietet und Kannibalismus vorbeugt: GD_G 2 ,[Kalk] da mit rein, ... Der bindet Wasser und die Hühner haben dadurch was zu tun."

\subsection{Tiergesundheit}

Tiergesundheit ist für die Landwirte ein wichtiger Indikator für Tierwohl. Vor dem Hintergrund der hohen biologischen Leistungen im Nutztierbereich stellt der Erhalt der Gesundheit eine wichtige Herausforderung dar, bei der noch Potenziale zur Optimierung gesehen werden. Ansatzpunkte zur Weiterentwicklung werden u.a. in der Zucht auf Vitalität und Robustheit gesehen. Teilweise wird eine Anpassung der Zuchtziele gewünscht, Schweinebereich: GD_S 2 „Ich sehe das nicht so als positiv, diese Entwicklung, dass da immer mehr und immer mehr Ferkel geboren werden müssen." Auf den Betrieben selbst werden Maßnahmen im Hygienemanagement als sinnvoll erachtet, um Krankheiten vorzubeugen und die Medikamentengabe zu reduzieren. Beispiele sind hygienische Maßnahmen in der Kälberfütterung, um Durchfall oder Flechten vorzubeugen, Maßnahmen in der
Liegeboxengestaltung bei Milchkühen oder insgesamt die Einrichtung von Krankenbuchten oder -abteilen, in denen kranke Tiere separat gehalten werden können: GD_R 1 ,Das ist ja oft das Problem, der Keimdruck, denn bei uns ist es Abkalbebox und Krankenbox ist eigentlich die gleiche Box." Die reine Stallhaltung wird gegenüber der Freiland- oder Auslaufhaltung als gesundheitsfördernd angesehen: GD_G 1 „Tiere, die bei nasser, kalter Witterung draußen sind, werden nun mal krank.“ Eine verbesserte Stallklimagestaltung mit Schadgasreduktionen wird befürwortet, um beispielsweise Atemwegserkrankungen zu vermeiden.

Für das Gesundheitsmanagement sehen die Landwirte einen Bedarf an technischen Lösungen zur Einzeltierbeobachtung bzw. Erfassung von Messwerten. Interessant wären technische Weiterentwicklungen zur kostengünstigen Erfassung tierbezogener Indikatoren, die frühzeitig Hinweise auf Unregelmäßigkeiten und Erkrankungen von einzelnen Tieren geben und dazu beitragen könnten, eine Ausbreitung in den Bestand zu vermeiden.

\subsection{Tierbetreuung}

Gerade vor dem Hintergrund der großen Tierbestände in den Betrieben kommt der Betreuung der Tiere eine wichtige Funktion im Hinblick auf das Tierwohl zu. Möglichkeiten zur Weiterentwicklung sehen die Landwirte einerseits im Verhalten der tierbetreuenden Personen und andererseits im Einsatz von Technik und in der Unterstützung durch Spezialisten, z.B. in der Klauenpflege bei Rindern. Die Betreuungsqualität der tierbetreuenden Personen machen die Landwirte an persönlichen Voraussetzungen fest: GD_R 1 „Du brauchst dieses Gefühl für Tiere.“ - „Also da brauchst du wirklich Leidenschaft für, und entweder man hat die oder man hat die nicht. Das kann in großen Betrieben sein, das kann in kleinen Betrieben sein, das ist völlig egal.“ GD_G 1 „Es gibt welche, die merken, dass die Tiere morgen krank werden und welche, dass die Tiere vorgestern schon krank waren." Besonders beim Einsatz von Fremdarbeitskräften sehen die Landwirte einen hohen Bedarf, diese Mitarbeiter im Hinblick auf Tierbeobachtung und Verhalten gegenüber den Tieren zu schulen: GD_G 2 ,Setze dich mal ruhig eine halbe Stunde hin, wie verhalten die Tiere sich, wenn sie stehen? Was passiert da alles, was hört man, was riecht man?“

Der Einsatz von Technik und Automatisierung wird von den Landwirten mit Blick auf die Tierbetreuung mehrheitlich als förderlich und zukunftsweisend angesehen. Einerseits werden 
durch die Technik Freiräume geschaffen, die zur Tierbeobachtung genutzt werden können: GD_S 1 „Das verbinde ich unmittelbar damit, dass wir mit technischem Fortschritt und über die Automatisierung z.B. bei Fütterung und anderen Dingen viel mehr Zeit bekommen haben, um wirklich die Tiere anzuschauen.“ GD_R 2 „Man braucht erst mal die Zeit, um das Auge auf das Tier richten zu können. Ab einer gewissen Betriebsgröße ist man mit Füttern und Melken so beschäftigt, dass man das einzelne Tier gar nicht so wirklich beobachten kann, da schaut man, dass man im Stall fertig wird. Da hilft mir die Technik weiter, um einfach Freiheiten zu kriegen.“ Zusätzlich kann die Technik exakte Messwerte zum Tierverhalten und zum Gesundheitsstatus liefern, die für die Versorgung und Betreuung genutzt werden können. Hier sehen die Landwirte für die Zukunft noch weiteres Potenzial: GD_S 1 „In Zukunft wird wahrscheinlich wichtig für uns werden, elektronische Hilfsmittel zur Verfügung zu nehmen.“

\subsection{Management}

Zur Weiterentwicklung des betrieblichen Managements mit Blick auf mehr Tierwohl sprechen sich die Tierhalter insbesondere für einen verbesserten innerbetrieblichen und überbetrieblichen Informationsaustausch aus. Innerbetrieblich geht es insbesondere darum, die Vielfalt der tierbezogenen Daten, die in den Betrieben erhoben werden, anwendungsorientiert zur Verfügung zu stellen und den Nutzern je nach Aufgabenbereich zuzuleiten. Vereinfachungen im Datenmanagement sollten letztlich dazu führen, den Betriebsleitern zielgerichtet eine Entscheidungshilfe zu bieten: GD_R 1 „Ich glaube es ist die Selektion von Informationen, wir haben so viele Informationen und man muss sich auf die wesentlichen konzentrieren, das ist, glaube ich, das größere Problem." Eine besondere Rolle kommt in diesem Zusammenhang auch der Beratung zu: GD_R 2 ,Die Beraterrolle die nimmt immer mehr. Vor 20 Jahren war es noch anders, weil jeder Betrieb so gewirtschaftet hat wie es immer war, und wenn es lief dann war schon alles richtig. "Die Fachgespräche mit außerbetrieblichen Personen oder Institutionen werden als wertvoll angesehen, um neue Ideen für den eigenen Betrieb zu generieren. Daneben ist den Landwirten der Austausch mit Berufskollegen zu Erfahrungen mit etablierten und weniger etablierten Haltungsverfahren sehr wichtig, um Perspektiven für die eigene betriebliche Zukunft auszuloten.

Ein weiterer wichtiger Punkt für mehr Tierwohl wird im Personalmanagement gesehen. Motivierte
Mitarbeiter werden als förderlich für die optimale Betreuung der Tiere betrachtet: GD_G 2 ,Wenn der Mitarbeiter motiviert ist geht er auch anders mit den Tieren um. " Hier werden ein regelmäßiger Austausch mit den Mitarbeitern sowie eine Wertschätzung der geleisteten Arbeit als förderlich angesehen. Daneben ist für die Landwirte auch die eigene Arbeitszeitstrukturierung wichtig, um sich auf den eigenen Betrieben für mehr Tierwohl einzusetzen: GD_R 1 „Dann kommt es ja auch noch darauf an wie viel Zeit er hat, um sich das alles anzugucken, was es an Informationen gibt."

\section{Diskussion}

Für die Landwirte ist die Weiterentwicklung von Tierhaltungsverfahren für mehr Tierwohl aufgrund der aktuellen gesellschaftlichen und politischen Diskussionen ein sehr präsentes Thema. Die gesellschaftliche Einstellung zum Tierwohl mit einem starken emotionalen Fokus ist auch für die Landwirte ein Aspekt des Tierwohlverständnisses und zeigt sich in Fürsorge, Verantwortung und teilweise Mitleid gegenüber den Tieren (Tab. 2). In einzelnen Fällen werden auch Schuldgefühle gegenüber den Tieren deutlich, z.B. im Umgang mit kranken oder besonders bedürftigen Tieren, wenn sie in Abwägung mit wirtschaftlichen Überlegungen nicht bestmöglich versorgt werden können.

In die Betrachtungsweise der Landwirte fließen daneben persönlich-identitätsbezogene Aspekte ein, die sie an ihrem Rollenverständnis und Berufsethos festmachen. Die diskutierten Maßnahmen für mehr Tierwohl orientieren sich im Wesentlichen an bisherigen und bereits etablierten Haltungsverfahren. Änderungen der Verfahren würden auch persönliche Veränderungen für die Tierhalter bedeuten und werden teilweise nicht als Weiterentwicklung sondern als Rückschritt gesehen. Das kann in den Augen der Landwirte zu Gesichtsverlust innerhalb der eigenen Branche gegenüber Berufskollegen führen. Loyalität und Anerkennung innerhalb der Branche sind offensichtlich wichtiger als Wertschätzung durch die Gesellschaft. Der Druck von außen verstärkt den inneren Zusammenhalt der Landwirte und führt zu einer starken Unterscheidung von Eigenund Fremdgruppe. Die Erhebungsmethode Gruppendiskussion zeigt die Dynamik einer sich verstärkenden Gruppenkohäsion deutlich auf.

Gleichwohl veranschaulichen die Ergebnisse auch die hohe Bedeutung der individuellen Arbeitszufriedenheit und -motivation für die tierbetreuenden Personen. Daraus leitet sich die Frage ab, inwieweit 
engagiertes Umsetzen innovativer Verfahren durch mutige Pioniere und gegenseitiges Lernen dazu beitragen können, das Beharrungsvermögen in der Branche aufzubrechen.

Mehr Tierwohl hat eine Chance in Betrieben umgesetzt zu werden, wenn dadurch das persönliche Erfolgsempfinden der Betriebsleiter und der objektiv messbare Betriebserfolg gesteigert werden können. Aufgrund langer Abschreibungszeiträume und der Spezifität der Investitionen mit teilweise unflexiblen Nutzungsalternativen kommen viele Ansätze für die Landwirte aus betrieblicher Sicht nicht in Frage. Aktuelle und neue gesetzliche Vorgaben und Richtlinien werden als Risikofaktor gesehen, da sie von gesellschaftlichen Trends und von der jeweils regierenden politischen Mehrheit beeinflusst und zudem bundesländerspezifisch ausgestaltet werden.

Tab. 2 Weiterentwicklung der Haltungsverfahren in unterschiedlichen Kontexten für Landwirte

\begin{tabular}{lll}
\hline Fokus & Sichtweise & $\begin{array}{l}\text { Bewertungs- und } \\
\text { Einflusskriterien }\end{array}$ \\
\hline Tier/ & emotional-relational & Verantwortung \\
Herde & & Fürsorge \\
& Mitleid \\
& & Schuldgefühle \\
Tierhalter persönlich- & Arbeitszufriedenheit \\
& identitätsbezogen & Rollen- bzw. \\
& & Aufgabenverständnis \\
& & Kohäsion der Berufskollegen \\
& & Wertschätzung durch die \\
& & Gesellschaft \\
Betrieb & ökonomisch-rational & Betriebserfolg \\
& & Einkommen \\
& & Sicherheit \\
& & Perspektive \\
\hline
\end{tabular}

Landwirte können derzeit nur schwer Offenheit für neue Ideen zu Tierhaltungsverfahren mit mehr Tierwohl, losgelöst von wirtschaftlichen und politischen Rahmenbedingungen, zeigen. Teilweise halten sie sich in den Gruppendiskussionen auch aus strategischen Gründen mit Vorschlägen zurück, weil sie befürchten, dass innovative Vorstöße schnell zu einer künftigen Anforderung werden. Neue Entwicklungen aufzuhalten bedeutet für die Landwirte, eigene unternehmerische Freiheiten $\mathrm{zu}$ bewahren und $\mathrm{zu}$ schützen. Durch den Verweis auf aktuell geltende Rahmenbedingungen schränken Landwirte jedoch ihren eigenen Handlungs- und
Verantwortungsbereich ein und delegieren die Verantwortung für Tierwohl an Politik und Marktpartner.

Damit politische Auseinandersetzungen und der interne Wettbewerb des Handels nicht auf dem Rücken landwirtschaftlicher Betriebe und Betriebsleiter ausgetragen werden, wären neue Mechanismen notwendig, Tierwohl einerseits transparent und objektiv zu bewerten und andererseits im Sinne eines öffentlichen Gutes durch Beratung, aber vor allem durch neue politische Maßnahmen und Instrumente, organisatorisch und finanziell $\mathrm{zu}$ unterstützen. Eine entsprechende Bewertung des Tierwohls, die weniger an der technischen Gestaltung von Tierhaltungsverfahren an sich, sondern stattdessen an den Tieren selbst orientiert ist, würde eine Verschiebung von ordnungspolitischer $\mathrm{zu}$ ergebnisorientierter Betrachtung des Tierwohls bedeuten. Der Maßstab für Tierwohl würde sich damit von einer input-basierten (was wird dem Tier geboten) zu einer output-basierten (welches Ergebnis wird für das Tier erzielt) Bewertung verschieben. Durch dynamische Innovationsanreize würden Landwirte in ihrer Schlüsselposition und ihrer Verantwortung für das Tierwohl gestärkt, weil Tierwohlziele sich auf ganz unterschiedlichen Wegen technisch und organisatorisch erreichen lassen. Gleichzeitig würde dadurch die unternehmerische Freiheit der Tierhalter gesichert und sogar erweitert werden. Die Grundlagen für neue Bewertungsansätze des Tierwohls bei verschiedenen Tierarten und die Akzeptanz dieser Ansätze bei Landwirten sollten Gegenstand künftiger Forschung zur Unterstützung politischer Entscheidungen sein.

\section{Literatur}

Bock BB, Van Huik MM, Prutzer M, Eveillard FK, Dockes A (2007) Famers' relationship with different animals: the importance of getting close to the animals. Case studies of French, Swedish and Dutch cattle, pig and poultry farmers. Int J Sociol Anthropol 15(3):108-125

Busch G, Kayser M, Spiller A (2013) „Massentierhaltung “ aus VerbraucherInnensicht - Assoziationen und Einstellungen. Jahrbuch der Österreichischen Gesellschaft für Agrarökonomie, 22(1):61-70

Heise H, Theuvsen L (2015) Biological Functioning, Natural Living oder Welfare-Quality: Untersuchungen zum Tierwohlverständnis deutscher Landwirte. Ber ueber Landwirtsch 93(3) 
Mayring P (2002) Einführung in die Qualitative Sozialforschung. Eine Anleitung $\mathrm{zu}$ qualitativem Denken. Beltz Verlag, Weinheim und Basel

Spooner JM, Schuppli CA, Fraser D (2014) Attitudes of Canadian Pig Producers Toward Animal Welfare. J Agric Environ Ethics 27(4):569-589

Statistische Ämter des Bundes und der Länder (2011) Agrarstrukturen in Deutschland. Einheit in Vielfalt. https://www.destatis.de/DE/Publikationen/Themati sch/LandForstwirtschaft/Landwirtschaftzaehlung/ AgrarstruktureninDeutschland5411203109004.pdf? blob=publicationFile

Te Velde H, Aarts N, van Woerkum C (2002) Dealing with ambivalence: Farmers' and consumers' perception of animal welfare in livestock breeding. J Agric Environ Ethics 15:203-219

Vanhonacker F, Verbeke W, van Poucke E, Pieniak Z, Nijs G, Tuyttens F. (2012) The concept of farm animal welfare: citizen perceptions and stakeholder opinion in flanders, Belgium. J Agric Environ Ethics 25(1):79-101
Van Huik MM, Bock BB (2007) Attitudes of Dutch pig farmers towards animal welfare, BRIT FOOD J Vol. 109 Iss 11 pp. 879 - 890

Waiblinger S (1996) Die Mensch-Tier-Beziehung bei der Laufstallhaltung von behornten Milchkühen. Witzenhausen

WBA Wissenschaftlicher Beirat Agrarpolitik beim BMEL (2015) Wege zu einer gesellschaftlich akzeptierten Nutztierhaltung. Gutachten. Berlin

Zander K, Isermeyer F, Bürgelt D, Christoph-Schulz I, Salamon P, Weible D (2013) Erwartungen der Gesellschaft an die Landwirtschaft. Münster: Stiftung Westfälische Landwirtschaft

Zapf R, Schultheiß U, Achilles W, Schrader L, Knierim U, Herrmann HJ, Brinkmann J, Winckler, C (2015) Tierschutzindikatoren: Vorschläge für die betriebliche Eigenkontrolle. Darmstadt: Kuratorium für Technik und Bauwesen in der Landwirtschaft 


\section{Standards, Hindernisse und Wünsche in der} Nutztierhaltung - Die Perspektive des Handels

Caspar Krampe ${ }^{1}$, Nadine Gier ${ }^{1}$, Julia Römhild ${ }^{1}$, Peter Kenning ${ }^{1}$

${ }^{1}$ Lehrstuhl für Betriebswirtschaftslehre, insb. Marketing, Heinrich-Heine-Universität, Universitätsstraße 1, 40225 Düsseldorf, Gebäude 24.21, Deutschland 0211-81-10278

caspar.krampe@hhu.de

\section{Einleitung und Zielsetzung}

Zwischen der Wunschvorstellung und der aktuellen Wahrnehmung der Nutztierhaltung durch die Gesellschaft zeigt sich meist eine hohe Diskrepanz (Zander et al. 2013). Diese lässt sich insbesondere bei den Positionen der verschiedenen Akteure bei der Durchsetzung höherer Tierwohlstandards beobachten (Zander et al. 2013). Zudem scheinen die in den Medien geführten Diskussionen und veröffentlichten Berichte rund um das Thema „Tierwohl“ maßgeblich das Meinungsbild in den verschiedenen gesellschaftlichen Gruppen zu prägen, zu polarisieren und deren Sensibilitäten zu erhöhen (Zander et al. 2013). Auf diese Entwicklung muss auch der an der Vermarktung, tierischer Erzeugnisse maßgeblich beteiligte Lebensmitteleinzelhandel (LEH) reagieren. Er hat dabei sogar eine zentrale Rolle, steht er doch im Spannungsfeld zwischen den Wunschvorstellungen der Verbraucher und den auf den Höfen sowie in den Zulieferbetrieben realisierbaren Bedingungen. Zudem ist der LEH dem Wettbewerb innerhalb des Marktes ausgesetzt, indem innovative (Marketing-)Maßnahmen einzelner Unternehmen hochgradig risikobehaftet sind und dementsprechend $\mathrm{zu}$ hohen ökonomischen Einbußen führen können. Aber auch ein gemeinsames, koordiniertes Vorgehen mehrerer Unternehmen für höhere Tierwohlstandards scheint für den Handel aus verschiedenen Gründen problematisch, nicht zuletzt deswegen, weil diese durch wettbewerbsrechtliche Bestimmungen beschränkt werden.

Vor diesem Hintergrund ist es Ziel des vorliegenden Teilbeitrags, den Handel als zentralen Akteur der Lebensmittelwirtschaft genauer in den Blick zu nehmen. Von besonderer Relevanz sind dabei, der Zielstellung des SocialLab Verbundprojektes entsprechend, die Motive und/oder Hindernisse für die Produkteinführung und -listung von Produkten mit höheren Tierwohlstandards. Konkret soll gezeigt werden, wie der Prozess der Listungsentscheidung in diesem Zusammenhang strukturiert ist, welche Meinungen im LEH zu verbesserten Tierwohlstands vorherrschen und welche Position im Hinblick auf etwaige regulierende Maßnahmen den LEH prägen. Zudem soll die Sicht des LEH auf den in der Diskussion stehenden informationsökonomischen Nutzen eines oder mehrerer Labels („Tierwohllabel“) ermittelt werden. Die so gewonnenen Ergebnisse sollen einen Beitrag dazu leisten, etwaige Hindernisse im Hinblick auf Maßnahmen zu identifizieren, mit denen gegebenenfalls ein höherer Tierschutzstandard realisiert werden könnte.

\section{Methode und Interviewleitfaden}

Um die skizzierte Zielstellung zu erreichen und um die Perspektive des LEH wissenschaftlich entsprechend differenziert $\mathrm{zu}$ durchdringen, wurde ein qualitativer Studienansatz gewählt. Der Aufbau und der Verlauf der Studie wurden dem typischen Aufbau ähnlicher Marktforschungsstudien (Kuß, Wildner, \& Kreis 2014) entsprechend in folgende sechs Schritte unterteilt:

1. Festlegung der Untersuchungsziele,

2. Festlegung des Untersuchungsdesigns,

3. Entwicklung des Messinstrumentariums,

4. Durchführung der Erhebung,

5. Datenanalyse und -auswertung und

6. Formulierung des Ergebnisberichts.

Um die gewünschten Erkenntnisse zu gewinnen wurden 15 Experteninterviews mit Top-Entscheidern im Bereich Vertrieb und Einkauf im LEH sowie weiteren handelsnahen Akteuren durchgeführt. Die aufgezeichneten Mitschnitte der Experteninterviews wurden für die weitere Datenanalyse sowie die damit verbundene Auswertung transkribiert (vgl. Höld, 2009; Mayring \& Fenzl, 2014). Die kommunizierten Daten wurden hierfür weitgehend von Dialekten befreit, an die Standardsprache angepasst und - wo geboten - in der Grammatik korrigiert, wodurch jedoch keine interpretativen Veränderungen des Ausgangsmaterials vorgenommen wurden. Aufgrund der besonderen Sensibilität der erhobenen Daten wurden alle mitgeschnittenen Tonaufnahmen ausschließlich universitätsintern und vertraulich von den Projektmitarbeitern transkribiert und vor der Inhaltsanalyse erneut zur Kontrolle an die Interviewpartner versendet. Zur strukturierten Inhaltsanalyse wurde die Software MaxQDA in der Version 12 verwendet (http://www.maxqda.de/). Diese etablierte Software ermöglicht die Zuordnung von 
Textstellen („Codierung“) zu den a priori definierten Kernthemen („Codes“), welche die Grundlage der hier angewandten qualitativ-strukturierenden Inhaltsanalyse darstellen.

Von besonderer methodischer Bedeutung für die Studie war darüber hinaus der Interviewleitfaden, welcher der Expertenbefragung als Messinstrument zugrunde lag. In diesem wurden vier Kernthemen, die der oben genannten Zielstellung entsprachen ausgearbeitet und im Experteninterview thematisiert. Die erste Kernthematik umfasste den Prozess der Listungsentscheidung im LEH. Ziel war die
Regulierung bei der Durchsetzung höherer Tierwohlschutzstandards. Das Ziel dieser Thematik war es, Hinweise auf das Meinungsbild bzw. die Einstellungen des LEH zu einer staatlichen Regulierung im Bereich der Fleischware abzubilden. Die vierte und letzte Kernthematik widmete sich der Wahrnehmung der Experten zum Einfluss von Labeln auf die Verbraucherentscheidung am Point-of-Sale sowie die damit verbundene Produktwahrnehmung. Abbildung 1 visualisiert die entsprechende Struktur des Gesprächsleitfadens noch einmal.

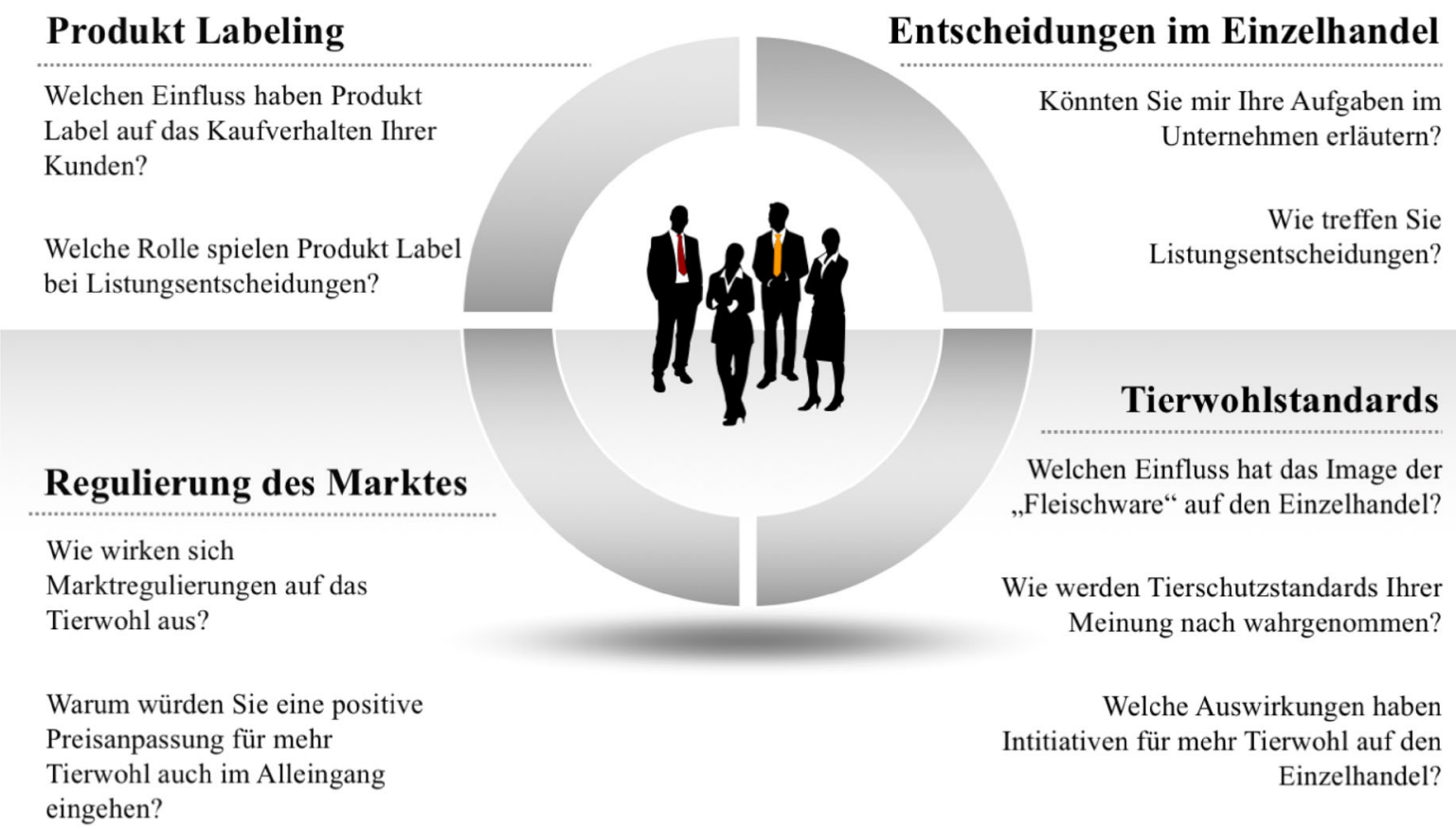

Abb. 1 Darstellung der vier Kernthemen sowie der untergeordneten Leitfragen. In den Experteninterviews wurden die Themen Listungsentscheidungen, Tierwohlstandards, Regulierung des Marktes und Produkt Labeling anhand eines strukturierten Gesprächsleitfadens besprochen

Identifikation der jeweils zugrundliegenden Entscheidungsprozesse. Zudem sollte beforscht werden, auf welchen Entscheidungsmustern und gegebenenfalls Heuristiken die Listungsentscheidung beruht. Die zweite Kernthematik des erstellten Interviewleitfadens umfasste den gesellschaftlichen und politischen Wunsch nach einer Integration höherer Tierwohlstandards in die Wertschöpfungskette. In diesem Zusammenhang wurde unter anderem die Wahrnehmung von Tierwohlstandards durch die Verbraucher aus Sicht der Experten thematisiert. Die dritte Kernthematik umfasste die Wahrnehmung der Experten zur staatlichen

\section{Die Kernthemen - Ausgewählte Ergebnisse}

Aufbauend auf den transkribierten Experteninterviews wurde eine qualitativ-deskriptive Begutachtung der Daten vorgenommen (Abb. 2). Es zeigte sich, dass Aspekte rund um das Thema Tierwohlstandards einen großen Teil der kodierten Daten ausmachten. Es sollte jedoch bei der nun folgenden strukturierten Inhaltsanalyse beachtet werden, dass es sich hierbei um eine aggregierte Darstellung der Ergebnisse handelt. Diese bildet zwar das generelle Meinungsbild der interviewten Experten ab, vernachlässigt jedoch gegebenenfalls abweichende 
Einzelmeinungen. Prägnante Aussagen der interviewten Experten des LEH sowie der handelsnahen Akteure werden hierbei skizziert und sollen einen Eindruck der Sichtweise der interviewten Experten vermitteln.

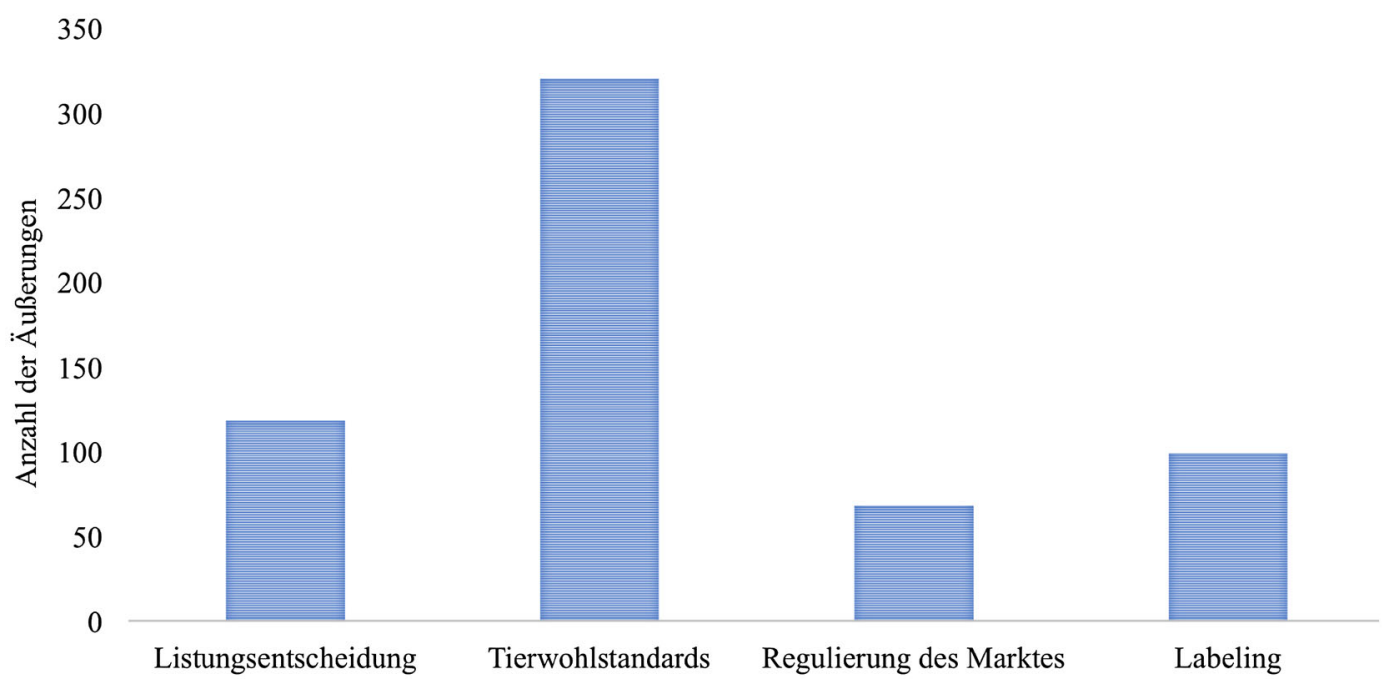

Abb. 2 Darstellung der Anzahl von Äußerungen zu den 4 Kernthemen. Für die inhaltliche Analyse wurden die transkribierten Interviews entsprechend der Kernthemen codiert und ausgewertet

Im Folgenden sollen nun die mit den oben skizzierten vier Thematiken bzw. Zielstellungen verbundenen Ergebnisse präsentiert werden. Hierzu wird jeweils zunächst die gesamte Anzahl der zurechenbaren Äußerungen genannt bevor dann jeweils die zentralen inhaltlichen Aspekte dargestellt werden.

\subsection{Listungsentscheidungen - Stabilität durch Vertrauen!}

Grundlage der im Hinblick auf die erste Thematik der Listungsentscheidung im LEH angewandten strukturierenden Inhaltsanalyse sind $n=118$ Äußerungen („Codierungen“), welche aus 6 Experteninterviews extrahiert und analysiert wurden.

Die Ergebnisse der Inhaltsanalyse zeigen in diesem Bereich der Untersuchung, dass Unternehmen des LEH oftmals auf langfristige, stetige und geprüfte Partnerschaften zurückgreifen, wodurch der „,sorglose“, transaktionskostenarme Fleischverkauf und konsum auf Seiten des LEH und des Verbrauchers gewährleistet werden soll. Demnach werden Listungsentscheidungen zumeist, nach einem primären, „checklisten-geprüften“ Auswahlverfahren, in enger Zusammenarbeit mit bewährten Partnern beziehungsweise Lieferanten getroffen.
Hierbei werden neue Produkte dem LEH zum einem vom Lieferanten vorgeschlagen (,Push-Ansatz“), zum anderem werden aber auch bestimmte Produkte bei den jeweiligen Lieferanten vom LEH angefragt („PullAnsatz $\left.{ }^{\star}\right)$. In diesem Zusammenhang scheinen das 
einstellt zu einer Reduktion von Transaktionskosten führt. Etablierte Anbieter sollten es daher tendenziell leichter haben, innovative Produkte im Regal zu platzieren.

„[Es] ergeben sich [...] Listungsentscheidungen [...] auf Basis von Vorschlägen, die uns die Lieferanten, mit denen wir seit vielen Jahren in der Regel vertrauensvoll zusammenarbeiten, [zukommen lassen]. "

\subsection{Die Integration von Tierwohlstandards - der mündige Verbraucher?}

Grundlage der im Kontext der Verbraucherwahrnehmung angewandten strukturierten Inhaltsanalyse waren $\mathrm{n}=91$ Äußerungen („Codierungen“), welche aus 9 Experteninterviews extrahiert und analysiert wurden.

\section{„Fleisch und Wurst sind Kulturgut.“}

Aus Handelssicht nehmen Fleischwaren nach wie vor einen hohen Stellenwert in der Wahrnehmung der Verbraucher ein. Als Nachweis dient den interviewten Experten des LEH oft der Ausgabenvergleich der Verbraucher für Fleischwaren im Vergleich $\mathrm{zu}$ anderen Produktgruppen. Insgesamt wird das Thema „Tierwohl“ als fester Bestandteil der gesellschaftlichen Auseinandersetzung gesehen. Zudem geht der Handel offenbar von einem differenzierten Verbraucherbild aus. Überwiegend werden Verbraucher aber als hinreichend mündig angesehen, um „am Ende des Tages das [zu] wählen, was für sie am besten ist“. Dementsprechend begreift sich der LEH lediglich als Anbieter von verschiedenen Wahloptionen, jedoch nicht als Vorentscheider über Moral und Verantwortung; der Wille des Kunden bleibt zumindest vordergründig frei. Trotzdem, scheinen sich die interviewten Experten des LEH sehr wohl bewusst über das tatsächliche Einkaufsverhalten der Verbraucher zu sein. So wird davon ausgegangen, dass viele Verbraucher auf die Produkte zurückgreifen, welche routiniert gekauft werden. Tierwohlaspekte spielen dabei eine untergeordnete Rolle, da der Verbraucher nach Wahrnehmung der Experten des LEH stark preisgetrieben ist. Es werden zudem Unterschiede zwischen Tierarten ausgemacht. So wird angenommen, dass die kritische Wahrnehmung der Verbraucher zur Rinderhaltung im Vergleich zur Schweine- und/oder Geflügelhaltung als weniger problematisch angesehen wird. Insgesamt stehen die interviewten Experten des LEH der Aussage, dass Verbraucher eine höhere Zahlungsbereitschaft für „Tierwohl-Produkte“ haben, kritisch gegenüber. So wird angenommen, dass die einfache Äußerung der Verbraucher, mehr für „Tierwohl“ bezahlen zu wollen, nicht ohne Weiteres zu einer Einstellungsänderung führt, sondern eventuell auf die soziale Erwünschtheit zurückzuführen ist.

„Die Moral endet am Regal.“

Im Fortgang der Gesprächsthematik mahnen die Händler zudem an, dass das Thema „Tierwohl“, als ein sehr emotionales Thema, im gesellschaftlichen Kontext nicht immer sachgerecht diskutiert wird. So weisen die Experten des LEH die Rolle des Regulators beziehungsweise Gatekeepers zurück und verweisen auf den mündigen Verbraucher. Gründe für das gesteigerte Interesse am „Tierwohl“ innerhalb der Gesellschaft macht der LEH an gesellschaftlichen Strömungen und Trends wie dem Vegetarismus, dem Veganismus, dem Flexitarismus oder der Profilierung bestimmter Gesellschaftsschichten durch "gutes“, meist biologisch erzeugtes Essen fest.

Zusammenfassend verweisen die Experten des LEH darauf, dass die Integration von Tierwohlstandards in die Wertschöpfungskette größtenteils durch das Einkaufsverhalten der mündigen Verbraucher (,PullAnsatz“) bestimmt wird, wobei angenommen wird, dass die reale Kaufentscheidung im Gegensatz zu regelmäßigen Bekundungen nach wie vor stark preisgetrieben ist und im ständigen Vergleich zu Konkurrenzangeboten steht. Ergänzend wird angenommen, dass der soziale Hintergrund ebenfalls einen Einfluss auf den Verbraucher und die damit verbundene Kaufentscheidung im Bereich der Fleischware hat. Das folgende Zitat gibt diesen Zusammenhang zutreffend wieder:

,[...] Es gibt sicherlich viele Familien [...], die sich [...] keine enorme Preissteigerung im Bereich Lebensmittel leisten können."

\subsection{Die Regulierung des Marktes - Fluch oder Segen?}

Grundlage der in dieser Thematik angewandten strukturierten Inhaltsanalyse waren $n=68$ Äußerungen („Codierungen“), die aus 8 Experteninterviews extrahiert und analysiert wurden. In der Diskussion um eine staatliche Regulierung von Tierwohlstandards zeichnet sich ein differenziertes Meinungsbild ab. Generell sehen die Experten des LEH die staatliche Regulierung des Marktes durch die Politik kritisch. Vielmehr betrachtet man die Möglichkeiten zur Selbstregulierung des Marktes als effizient und ausreichend. Dennoch wurden durchaus Vorteile einer Gesetzgebung für mehr „Tierwohl“ erkannt. So 
werden bestehende Gesetzte zur Verbesserung der Tierhaltung gelobt, die Umsetzung und Kontrolle dieser aber bemängelt. Die befragten Experten fordern darum eine fundierte ,weniger populistisch orientierte“ Diskussion über mögliche Maßnahmen, die zu einer weiteren Verbesserung des „Tierwohls“ führen können. Des Weiteren bemängeln die interviewten Experten des LEH das zum Teil unkoordinierte Vorgehen von Bund und Ländern bei der Gesetzgebung im Bereich der Tierhaltung. Zudem wird die Regulierung des Marktes auf nationaler Ebene (für geltendes nationales Recht) kritisch gesehen da diese, in Zeiten der Globalisierung/Europäisierung, nicht immer mit dem europäischen Recht vereinbar und durchsetzbar ist. Ein in diesem Zusammenhang bezeichnendes Zitat ist das folgende:

„Wir leben ja schließlich nicht auf einer Insel.“

\subsection{Labeling - Überforderung am Point-of-Sale?!}

Grundlage der im Zusammenhang mit der „Labeldiskussion“ angewandten strukturierten Inhaltsanalyse waren $n=24$ Äußerungen („Codierungen“), welche aus 6 Experteninterviews extrahiert und analysiert wurden.

\section{„Der Kunde wird überfrachtet.“}

Maßnahmen zum Tierschutz werden durch die Experten des Lebensmitteleinzelhandels und weiteren handelsnahen Akteure primär als informationsökonomisch motiviert und verbraucherorientiert wahrgenommen. Dabei wird kritisiert, dass der Kunde durch die Vielzahl von Labels am Point-of-Sale mittlerweile überfordert ist und/oder diese oftmals gar nicht wahrnimmt. Folglich werden entsprechende Ansätze und Kommunikationsmaßnahmen als kontraproduktiv beschrieben, da viele Labels in direkter Konkurrenz zu einander stehen. Als Beispiel werden vom LEH kreierte „Biosiegel“ genannt, welche in direkter Konkurrenz mit „Biosiegeln“ des Wettbewerbers sowie der nationalen und europäischen Kennzeichnung von biologischen Erzeugnissen stehen. Alternativen zu dem bisherigen System der Verbraucherinformation sind daher wünschenswert (vgl. hierzu Kapitel „Zur Konzeption eines Verbraucherinformationssystems als Ergänzung - oder Alternative? - zum klassischen Informationslabel“).

\section{Zusammenfassung und Ausblick}

In Experteninterviews mit Vertretern des Lebensmitteleinzelhandels sowie den weiteren handelsnahen
Akteuren konnte die Perspektive des Handels wissenschaftlich durchdrungen werden und in Bezug auf das Thema „Tierwohlstandards“ Motivationen und Hemmnisse der Durchsetzung identifiziert werden. Hierzu wurden 4 verschiedene Themenfelder besprochen. Im Hinblick auf die Listungsentscheidung lässt sich festhalten, dass der Handel versucht, durch standarisierte Prüfungen seiner Lieferanten einen unkritischen Fleischverkauf und -konsum zu gewährleisten und darüber hinaus auf langfristige Partnerschaften vertraut. Bezüglich der Sortimentsvielfalt und Auswahl an Produkten orientiert der Handel sich vor allem am Kaufverhalten der Verbraucher und sieht sich als Bereitsteller der Wahloptionen und nicht als moralischen „Wahlhelfer“ der Verbraucher. Im Themenfeld der „Regulierung“ werden zwar Vorteile durch umsichtige und inkrementelle Regulierungen bei den Tierwohlstandards gesehen, jedoch wird der Markt zumeist als effektives System betrachtet. Staatliche und selbstständige Label stellen ein Beispiel für solche Regulierungen dar, scheinen aber (auch) nach Ansicht des Handels Gefahr zu laufen, ihr Ziel durch die Überlastung der Verbraucher mit zusätzlichen Informationen zu verfehlen.

Die folgenden Ausführungen sind zum Teil das Ergebnis einer umfassenden Diskussion der Autoren mit verschiedenen verbraucherpolitisch aktiven Wissenschaftlern, denen wir zu Dank verpflichtet sind. Er gilt allen Projektpartnern des Verbundprojektes „SocialLab - Nutztierhaltung im Spiegel der Gesellschaft“, insbesondere den Kollegen der Fachhochschule Südwestfalen (Prof. Dr. Marcus Mergenthaler) und der Zeppelin Universität (Prof. Dr. Lucia Reisch). Des Weiteren möchten wir uns an dieser Stelle bei allen Interviewpartnern für ihre Teilnahme sowie die wertvollen Beiträge bedanken. Die Förderung des Vorhabens erfolgte aus Mitteln des Bundesministeriums für Ernährung und Landwirtschaft (BMEL) aufgrund eines Beschlusses des deutschen Bundestages. Die Projektträgerschaft erfolgte über die Bundesanstalt für Landwirtschaft und Ernährung (BLE) im Rahmen des Programms zur Innovationsförderung (FKZ: 2817203413).

\section{Literatur}

Gigerenzer G, Todd P M, ABC Research Group (1999) Simple heuristics that make us smart. Oxford University Press

Höld R (2009) Zur Transkription von Audiodaten. Qualitative Marktforschung, 655-668 
Kuß A, Wildner R, Kreis H (2014) Explorative Untersuchungen mit qualitativen Methoden Marktforschung, Springer, pp 51-62

Mayring P, Fenzl T (2014) Qualitative inhaltsanalyse Handbuch Methoden der empirischen Sozialforschung, Springer, pp 543-556

Selten R (2001) What is bounded rationality bounded rationality: the adaptive toolbox, 13,36
Zander K, Isermeyer F, Bürgelt D, Christoph-Schulz I, Salamon P, Weible D (2013) Erwartungen der Gesellschaft an die Landwirtschaft. Münster: Stiftung Westfälische Landschaft 
Adequate animal welfare standards from a citizen perspective - A stated choice experiment on broiler

\author{
Jutta Roosen ${ }^{1}$, Bernhard Kalkbrenner ${ }^{1}$, Silke Fischer ${ }^{1}$ \\ ${ }^{1}$ Chair of Marketing and Consumer Research, TUM \\ School of Management, Technical University of \\ Munich, Alte Akademie 16, 85354 Weihenstephan, \\ Germany, \\ jroosen@tum.de
}

\section{Introduction}

A number of laws are regulating animal rights in Germany. Among those is the regulation of Animal Protection and various specific regulations with regard to livestock. Despite this regulatory oversight, animal welfare in farm production has become a contentious issue. Concern is particularly high regarding pigs and poultry (see Christoph-Schulz et al. in this article). As a reaction, new regulations on animal welfare and labelling rules to enable consumer choice for higher animal welfare products are under discussion. At the same time, animal welfare labels gain significant market shares in some countries. For example, products marketed in The Netherlands under the Beter Leven label result in spending of 295.3 mio. $€$ (Smith and Pinckaers 2012). However, critics argue that voluntary labels will not suffice to address all issues of animal welfare (Blaha 2017). The adjustment in livestock husbandry standards is thus prone to possible, additional regulatory action.

Blaha (2017) names 4 areas of action that should be taken into account: keeping all farm animals in accordance with the animal protection law allowing for natural behaviour and avoiding amputations, active animal health management for all livestock units, health monitoring, and educating animal livestock owners and managers. However, higher animal welfare standards will come at a cost. A recent report by the Scientific Advisory Board on Agricultural Policy (2015) estimates that the implementation of their recommendations for a viable livestock husbandry accepted by large parts of the population would cost in the range of 3 to 5 bn. $€$ per year. Karl \& Noleppa (2017) report that German agriculture is subject to additional costs of 696.0 mio. $€$ for respecting EU regulations on animal welfare, food safety and animal health in German livestock production.

In order to understand the economic welfare implications of stricter production standards and to investigate which standards yield additional consumer benefits, estimates of willingness to pay (WTP) are needed. WTP is used to measure the value that a good or service provides to consumers and can be linked to economic welfare studies comparing costs and benefits of changes in the regulatory environment (Hanley et al. 2001). Consumers and citizens have specific concerns that need to be addressed to make livestock production acceptable in their view (Scientific Advisory Board on Agricultural Policy 2015). This paper's objective is to investigate the different aspects characterizing broiler production practices that lead to concern among consumers and to estimate WTP for changes in their regulation. The results are informative for future cost-benefit analyses of higher welfare standards and for organizations designing label requirements to ensure that their labels meet required demand levels in the market place. In this study, we focus on the WTP for changes in the production of broilers. The number of broilers in Germany is estimated at 97.1 mio. in 2013 (Bundesministerium für Ernährung und Landwirtschaft 2016). The average number of animals per production unit is 21.588 (Bundesministerium für Ernährung und Landwirtschaft 2016). Around 77 \% of broilers are produced in units of 50.000 animals and more. These units represent about $13 \%$ of broiler farms in Germany.

There is a significant number of WTP studies for animal welfare. Meta-analyses have been provided by Norwood \& Lusk (2011), Lagerkvist and Hess (2011) and Clark et al. (2017). Clark et al. (2017) find a positive WTP for improved animal welfare independent of species and welfare aspects. Reviewing studies of WTP for avoiding specific technologies Lusk et al. (2014) find significant heterogeneity in WTP, generally increasing with the income and decreasing with age. A stated choice study on broiler filets in the Netherlands by de Jonge et al. (2015) included product attributes such as the Beter Leven hallmark, outdoor access, stocking density and day-night rhythm. They find heterogeneous preferences, i.e. different consumer segments that state varying WTP for broiler filets with higher animal welfare standards. Carlsson et al. (2007) analyzed WTP for animal welfare for broilers in Sweden. A preference for keeping broilers outdoors (in summer)/in smaller groups indoors (in winter) was found, as well as a WTP for slow growth breeds (as compared to fast growing breeds) in forced choices. No WTP for mobile abattoirs over transport of animals to slaughter houses was found. For Germany, Heise and Theuvsen (2017) recently examined the general willingness to pay for meat, eggs and milk in Germany. 
Their analyses show that $76.5 \%$ of German consumers state a willingness to pay a price premium for higher animal welfare standards. On average, price premia up to $40 \%$ could be accepted, but only few consumers would pay premia above $40 \%$. The study finds that preferences are heterogeneous and factors such as gender and involvement influence the willingness to pay.

Against this background, the present study aims to investigate the following 3 questions:

- RQ 1: From a citizen perspective, who carries the responsibility for adequate animal welfare standards?

- RQ 2: What are consumer preferences for adequate regulations for animal welfare for broiler production?

- RQ 3: How do consumers react in their purchase behavior to an increasing price for meat?

\section{Methodology}

\subsection{Methods}

We conducted an online survey to investigate citizen preferences for improved animal welfare practices in broiler meat production. The survey included a choice experiment on the preferences towards welfare standards in different production systems for chicken and pigs. This paper focuses on the hypothetical choices as regards broilers. In contrast to many other studies, our survey focused on participants choice among different regulatory options that would apply to all broiler meat produced in Germany and eventually lead to higher prices. Before the choice experiment, we presented instructions, an introduction to the attributes, and the payment vehicle, i.e. new regulations would lead to higher meat prices. Each choice question represents a discrete choice between three legal environments with specific animal welfare standards and the status quo. Based on a literature review, we included 5 regulatory parameters with 2 levels each (maximum number of animal capacity per operating unit, stocking density, fattening period, housing system, and bill shortening) and 2 parameters with 4 (day-old chicken) and 5 levels (price). The attributes and levels are presented in Table 1 . Table 2 shows a sample choice task.

Table 1 Attributes and levels used in the choice experiments

\begin{tabular}{|c|c|c|c|c|c|}
\hline \multirow{2}{*}{$\begin{array}{l}\text { Attribut } \\
\text { Maximum number of } \\
\text { animal capacity }\end{array}$} & \multicolumn{5}{|l|}{ Levels } \\
\hline & $18.000^{a}$ & 5.000 & & & \\
\hline Stocking density & $\begin{array}{l}23 \text { Animals/ } \\
\mathrm{m}^{2 \mathrm{a}}\end{array}$ & 10 animals $/ \mathrm{m}^{2}$ & & & \\
\hline Fattening period & $\begin{array}{l}\text { Approx. } \\
32 \text { days }^{\mathrm{a}}\end{array}$ & Approx. 60 days & & & \\
\hline Housing system & Barn $^{a}$ & Free range & & & \\
\hline $\begin{array}{l}\text { Male chicks from } \\
\text { laying hens }\end{array}$ & $\begin{array}{l}\text { Day-old } \\
\text { chicks are } \\
\text { killed }^{\text {a }}\end{array}$ & $\begin{array}{l}\text { Use of spring chicken } \\
\text { (male laying hybrids) }\end{array}$ & $\begin{array}{l}\text { Breeding for mast and } \\
\text { laying (dual purpose breed) }\end{array}$ & $\begin{array}{l}\text { Gender sexing in the egg and } \\
\text { elimination of the male eggs }\end{array}$ & \\
\hline No bill shortening & $\begin{array}{l}\text { Bill } \\
\text { shortening }^{a}\end{array}$ & Without bill shortening & & & \\
\hline $\begin{array}{l}\text { Increase in monthly } \\
\text { food spending }\end{array}$ & $€ 0^{\mathrm{a}}$ & $€ 2.45$ & $€ 4.90$ & $€ 7.35$ & $€ 9.80$ \\
\hline
\end{tabular}

${ }^{a}$ Status quo option and reference level in the data analysis 
Table 2 Example of a choice set (translated from German)

Please choose one of the 4 alternatives:

Which scenario would you opt for considering the changes in your monthly food spending?

\begin{tabular}{|c|c|c|c|c|}
\hline & Scenario A & Scenario B & Scenario C & Status quo \\
\hline & Maximum no. of animal capacity: 18.000 & $\begin{array}{l}\text { Maximum no. of animal } \\
\text { capacity: } 18.000\end{array}$ & $\begin{array}{l}\text { Maximum no. of animal } \\
\text { capacity: } 5.000\end{array}$ & $\begin{array}{l}\text { No. revision of the } \\
\text { regulatory framework }\end{array}$ \\
\hline & 10 animals $/ \mathrm{m}^{2}$ & 10 animals $/ \mathrm{m}^{2}$ & 23 animals $/ \mathrm{m}^{2}$ & \\
\hline & Barn & Barn & Free range & \\
\hline & Fattening period approx. 32 days & $\begin{array}{l}\text { Fattening period approx. } \\
32 \text { days }\end{array}$ & $\begin{array}{l}\text { Fattening period approx. } \\
60 \text { days }\end{array}$ & \\
\hline & $\begin{array}{l}\text { Gender sexing in the egg and elimination } \\
\text { of the male eggs }\end{array}$ & $\begin{array}{l}\text { Use of spring chicken (male } \\
\text { laying hybrids) }\end{array}$ & Day-old chicks are killed & \\
\hline & Without bill shortening & Bill shortening & Without bill shortening & \\
\hline & Increase in monthly food spending $€ 9.80$ & $\begin{array}{l}\text { Increase in monthly food } \\
\text { spending } € 4.90\end{array}$ & $\begin{array}{l}\text { Increase in monthly food } \\
\text { spending } € 2.45\end{array}$ & \\
\hline I choose: & A & B & C & D \\
\hline
\end{tabular}

The scenarios are built on the current German regulations for chicken husbandry. The study focuses on 6 attributes and analyzes current animal welfare standards and higher standards. While the attributes refer mostly to broiler production per se, some attributes are based on the interlinkage to the production of layers, as certain regulations in one sector would have implications for the other. First, the size of the farm operation was defined as 18.000 chickens $^{\mathrm{a}}$ or 5.000 chickens (levels marked with a represent the status quo). 18.000 chickens represents a common size. Second, the stocking density is either 23 animals/ $\mathrm{m}^{2 \mathrm{a}}$, the current standard, or 10 animals $/ \mathrm{m}^{2}$. Third, as housing systems we included barn ${ }^{\mathrm{a}}$ and free-range. Fourth, the fattening period was defined as 32 or 60 days. Fifth, it is common to kill the day-old chicks of laying hens ${ }^{\mathrm{a}}$ after hatching since special breeds are used as broilers. We defined 3 alternatives for dealing with day-old chicks: the use of spring chicken (male laying hybrids used for meat at early age), breeding for mast and laying, and gender sexing in the egg with the elimination of the male eggs. Sixth, according to current regulations farmers are allowed to cut the bills ${ }^{\mathrm{a}}$ of the parent birds to prevent picking. Another practice is to not cut the bills. Price levels were defined as increases in household expenditures on food due to the higher costs of the different animal welfare standards. Compared to no change in the status quo $(0 €)$, we defined increases in monthly food spending as $+2.45 €,+4.90 €,+7.35 €$ and $+9.80 €$. The higher food spending is based on a percentage change from the average expenditures on food. According to the Income and Expenditure Survey
(Statistisches Bundesamt 2011) average household expenditures are $290 €$ per household and month for food, and $49 €$ for meat and meat products. The expenditure change scenarios thus correspond on average to a $5,10,15$ and $20 \%$ change in meat expenditures per household and month.

Using Ngene software we generated an 'optimal orthogonal in the difference' design (Street et al. 2005). We used a fractional design that resulted in 16 choice sets. As we blocked the sets into 2 blocks, respondents had to fill in one block with 8 choice sets. The resulting MNL D-error was 0.183.

\subsection{Data collection}

A total of 1420 respondents from Germany were interviewed by means of internet-based questionnaires in May and June 2016. A market research company was in charge of recruiting the respondents and conducting the survey. The panel is representative of the German population in terms of location, monthly net income, sex, age, employment status, and education. The total sample was split into one group filling in the chicken experiment, while the other group took part in an experiment on pigs. 714 respondents were allocated the choice experiment on chicken. The majority of the respondents (52.4\%) often purchases chicken meat, $42.4 \%$ rarely, and $5.2 \%(\mathrm{n}=37)$ do not buy chicken at all. In this article, we only include consumers who purchase chicken at least rarely. Hence, the final sample consisted of 677 respondents. Table 3 presents the composition of the total sample and the 
broiler sub-sample. The sub-sample has similar characteristics as the total sample.

Table 3 Sample Characteristics

\begin{tabular}{lll}
\hline Variables & Broiler sub- & Total sample \\
sample & $(N=1420)$ \\
& $(N=677)$ & \\
& Frequency $(\%)$ & Frequency \\
& & $(\%)$
\end{tabular}

\begin{tabular}{|c|c|c|}
\hline \multicolumn{3}{|l|}{ Gender } \\
\hline Female & 50.7 & 51.6 \\
\hline Male & 49.3 & 48.4 \\
\hline \multicolumn{3}{|l|}{ Age } \\
\hline $18-24$ years & 7.8 & 8.0 \\
\hline 25-29 years & 7.0 & 7.4 \\
\hline $30-39$ years & 17.5 & 18.0 \\
\hline $40-49$ years & 16.2 & 16.1 \\
\hline $50-64$ years & 27.9 & 27.9 \\
\hline 65 years and older & 23.5 & 22.6 \\
\hline \multicolumn{3}{|l|}{$\begin{array}{l}\text { Average monthly net household } \\
\text { income (in Euro) }\end{array}$} \\
\hline Less than 500 & 2.1 & 2.1 \\
\hline $500-899$ & 7.2 & 7.6 \\
\hline 900-1299 & 15.2 & 13.7 \\
\hline 1300-1499 & 8.6 & 8.5 \\
\hline $1500-1699$ & 7.5 & 7.3 \\
\hline 1700-1999 & 9.3 & 9.9 \\
\hline 2000-2599 & 16.2 & 16.0 \\
\hline 2600-3199 & 11.8 & 11.8 \\
\hline $3200-4499$ & 14.2 & 14.7 \\
\hline $4500-5999$ & 5.2 & 5.8 \\
\hline At least 6000 & 2.7 & 2.7 \\
\hline \multicolumn{3}{|l|}{ Education } \\
\hline No degree & 1.5 & 1.0 \\
\hline $\begin{array}{l}\text { Secondary general school- } \\
\text { leaving certificate } \\
\text { (Hauptschulabschluss) }\end{array}$ & 23.5 & 21.6 \\
\hline $\begin{array}{l}\text { Certificate of ten-grade school } \\
\text { of general education in the } \\
\text { former GDR (Polytechnische } \\
\text { Oberschule) }\end{array}$ & 6.6 & 6.2 \\
\hline $\begin{array}{l}\text { Intermediate school-leaving } \\
\text { certificate (Mittlere Reife) }\end{array}$ & 28.4 & 28.9 \\
\hline $\begin{array}{l}\text { University/University of applied } \\
\text { sciences entrance qualification } \\
\text { (Fachabitur, Abitur) }\end{array}$ & 21.1 & 21.4 \\
\hline $\begin{array}{l}\text { Degree from university or } \\
\text { university of applied sciences }\end{array}$ & 18.9 & 20.9 \\
\hline
\end{tabular}

\subsection{Statistical analysis}

We conducted a choice experiment, which builds on the random utility framework (McFadden 1974). To test for the assumption of independence of irrelevant alternatives (IIA) among choice options, we conducted a Hausman test (Hausman and McFadden 1984). The test results in significant outcomes and rejects the IIA assumption. We, hence, estimate a randomparameters logit model using Stata (commands: mixlogit and wtp). The data is dummy coded and the parameters in this study are set to be random, but price and the status quo option are defined as fixed (Hole and Kolstad 2012). We use 500 Halton draws to estimate our random parameters logit model.

\section{Results}

We asked respondents about (1) the responsibilities for ensuring adequate animal welfare standards, (2) conducted a choice experiment on broiler husbandry standards and (3) analyzed consumption responses to higher meat prices. Our respondents see mainly farmers $(43.3 \%)$ in charge of an adequate animal husbandry, followed by the state (26.8\%), and consumers themselves (21.5\%). ${ }^{12}$ Only $6.5 \%$ see the retail sector as mainly responsible to ensure adequate animal welfare. $2.2 \%$ of the sample selected "others" and most of them filled in that all the above-mentioned stakeholders are in charge. The results of the random parameters logit model of the choice experiment on different legal settings for farm animal welfare standards for chicken are presented in Table 4. The mean estimate coefficients show the utility attached to the different levels, and significant standard deviations represent preference heterogeneity.

\footnotetext{
${ }^{12}$ Results for the full sample $(\mathrm{n}=1420)$; Item: "In your opinion, who is mainly responsible for an adequate animal husbandry?"
} 
Table 4 Conditional Logit Parameter Estimates

\begin{tabular}{|c|c|c|}
\hline Variable & Coef. & $\begin{array}{l}\text { Std. } \\
\text { Err. }\end{array}$ \\
\hline \multicolumn{3}{|l|}{ Mean estimates } \\
\hline Status quo & $0.748^{* * *}$ & 0.107 \\
\hline Expenditures & $-0.158^{* * *}$ & 0.009 \\
\hline 3.000 animals (Reference: 18.000 animals) & 0.100 & 0.060 \\
\hline 10 animals $/ \mathrm{m}^{2}$ (Reference: 23 animals $/ \mathrm{m}^{2}$ ) & $0.638^{* * *}$ & 0.080 \\
\hline Free range (Reference: Barn) & $0.743^{* * *}$ & 0.068 \\
\hline $\begin{array}{l}\text { Approx. } 60 \text { days of fattening (Reference: } \\
\text { Approx. } 32 \text { days) }\end{array}$ & 0.111 & 0.065 \\
\hline \multicolumn{3}{|l|}{$\begin{array}{l}\text { Day-old chicken (reference: day-old chicks } \\
\text { are killed) }\end{array}$} \\
\hline Spring chicken (male laying hybrids) & $0.793 * * *$ & 0.084 \\
\hline Breeding for mast and laying & $1.135^{* * *}$ & 0.080 \\
\hline $\begin{array}{l}\text { Gender sexing in the egg and elimination of } \\
\text { the male eggs }\end{array}$ & $0.939 * * *$ & 0.093 \\
\hline $\begin{array}{l}\text { No bill shortening (reference: bill } \\
\text { shortening) }\end{array}$ & $0.840^{* * *}$ & 0.064 \\
\hline \multicolumn{3}{|l|}{ Standard deviations } \\
\hline 3.000 animals & $0.69^{* * *}$ & 0.089 \\
\hline 10 animals $/ \mathrm{m}^{2}$ & $1.562^{* * *}$ & 0.084 \\
\hline Free range & $1.073^{* * *}$ & 0.080 \\
\hline Approx. 60 days & $1.012^{* * *}$ & 0.074 \\
\hline Spring chicken (male laying hybrids) & $0.783^{* * *}$ & 0.104 \\
\hline Breeding for mast and laying & $0.991^{* * *}$ & 0.089 \\
\hline $\begin{array}{l}\text { Gender sexing in the egg and elimination of } \\
\text { the male eggs }\end{array}$ & $1.190^{* * *}$ & 0.096 \\
\hline No bill shortening & $1.126^{* * * *}$ & 0.069 \\
\hline
\end{tabular}

${ }^{*} \mathrm{p}<0.05,{ }^{* *} \mathrm{p}<0.01,{ }^{* * *} \mathrm{p}<0.001$

No. of observations $=21664$; LR chi $^{2}(8)=1536.72$; Log likelihood $=-6066.957$; Prob $>\mathrm{chi}^{2}=0.000$

All estimates are significant except the maximum number of animal capacity and the fattening period. However, the results of the standard deviations indicate that preference heterogeneity exists for all attributes; i.e. respondents value certain product attributes to varying degrees.

The results show a significant preference for the status quo. 51 participants chose the status quo in all 8 choice sets presented-consistently refusing regulatory revisions. The coefficient of expenditure is negative, indicating that consumers prefer lower expenditures to higher ones.

The maximum number of animal capacity and the fattening period do not have a significant effect on choices as regards higher animal welfare standards. We find a preference for a lower density of animals-a regulation with 10 animals $/ \mathrm{m}^{2}$ is preferred over 23 animals $/ \mathrm{m}^{2}$. The housing system is another significant parameter for animal welfare standards. Consumers state a preference for free range over barn housing. As male chicks of laying hens cannot be used as fattened chicken, different alternatives exist to deal with this issue. When compared to the status quo of killing the day-old chicks, consumers state preferences for breeding for mast and laying, followed by gender sexing in the egg and elimination of the male eggs, and the use of spring chicken (male laying hybrids). Finally, consumers advocate no shortening of bills.

In order to analyze the WTP of consumers, we examine an increase of food spending for higher farm animal welfare standards. We elicit relatively high price premia (in terms of higher food spending) for the issue of day-old chicks. The results indicate higher WTP for breeding for mast and laying $(+7.2 €)$, gender sexing in the egg and elimination of the male eggs $(+6.0 €)$, and spring chicken $(+5.0 €)$, i.e. male laying hybrids, when compared to killing day-old chicks. Consumers are found to be willing to increase their monthly spending by $5.3 €$ for animals welfare standards that prohibit shortening bills. The housing system is another important aspect analyzed in this study. We find additional monthly expenditures of $4.7 €$ for free-range chickens, when compared to barn housing. To achieve a regulation with lower densities of animals (10 animals $/ \mathrm{m}^{2}$ instead of 23 animals $/ \mathrm{m}^{2}$ ), consumers would be willing to spend additional 4.1€ per month on food.

Table 5 WTP estimates

Increase in monthly food spending (in $€$ )

Maximum number of animal capacity (reference: 18.000 animals)

5.000 animals $\quad 0.63$ (ns)

Density (reference: 23 animals $/ \mathrm{m}^{2}$ )

10 animals $/ \mathrm{m}^{2}$

4.05

Fattening period (reference:

Approx. 32 days)

approx. 60 days

0.70 (ns)

Housing system (reference: Barn)

Free range

4.71

Day-old chicken (reference:

day-old chicks are killed)

Spring chicken (male laying

hybrids)

Breeding for mast and laying $\quad 7.20$

Gender sexing in the egg and $\quad 5.96$

elimination of the male eggs

No bill shortening (reference: Bill 5.33 shortening)

$n s$ not statistically significant 
In order to verify the findings of the choice experiment, we asked respondents $(n=1420)$ about their purchasing behavior in case the price for meat would increase. Around half of the consumers (49.2\%) would not reduce their meat consumption while over a third (36.9 \%) would lower it $2.7 \%$ of the sample indicated to increase their meat consumption if higher animal welfare standards become the norm. A share of $11.2 \%$ do not know if they would change their behavior.

\section{Discussion}

The objective of this study was to investigate the different aspects characterizing broiler production practices that lead to concern among citizens and to estimate WTP for changes in regulations. We elicited preferences of German consumers for different regulations as regards broiler chicken using a stated choice experiment. With regard to RQ1, we find that-from a citizen perspective-farmers are assigned primary responsibility to ensure adequate animal welfare. The state and consumers themselves are seen as further important actors, while the role of the retail sector is viewed as almost negligible. These findings correspond to the study by Zander et al. (2013), with farmers being mainly responsible to ensure adequate animal husbandry, followed by the state. However, the present study indicates a more prominent role of the consumers-21.5\% see consumers as mainly in charge compared to $13.2 \%$ in Zander et al. (2013).

With respect to RQ2-consumer preferences for regulations for animal welfare for broiler production-the study shows that the killing of day-old chicks is an issue that consumers oppose. Strong preferences exist for breeding for mast and laying, followed by gender sexing in the egg with the elimination of the male eggs, and male laying hybrids. It must be noted that the survey was conducted in temporal proximity to the verdict of the Oberverwaltungsgericht NRW on the killing of one-day old chicks (20 A 488/15; 20 A 530/15). Bill shortening is another issue that consumers refuse as they state a relatively high willingness to pay for regulation prohibiting the shortening of bills. The housing system of the broiler is also relatively important, i.e. consumers value free range over barn housing. Finally, consumers value regulations enforcing lower densities (fewer animals $/ \mathrm{m}^{2}$ ). The maximum number of animal capacity and the length fattening period are not considered as significant parameters for animal welfare.

As indicated by the significant variation in the parameters, preferences for all attributes are heterogeneous. The size of the variation indicates that some consumers value the different attribute levels, while others do not. The willingness to pay parameters-measured in terms of a percentage change from the average expenditures on meat-for male laying hybrids, free range and lower stocking density are in the range of a $10 \%$ increase in meat expenditures. Preferences for gender sexing are slightly higher. Regulations stipulating breeding for mast and laying would be favored and a willingness to spend around $15 \%$ more per month on meat is found. In our hypothetical scenario, we find a relatively high WTP for dual-purpose chicken, but also see heterogeneous preferences. Overall, the increase in monthly food spending for meat between $10 \%$ and $15 \%$ can be regarded as realistic (from the consumer perspective) as e.g. Heise and Theuvsen (2017) found accepted premia for products with higher animal welfare standards of up to $40 \%$. However, the additional costs for chicken products with higher animal welfare standards needs to be investigated. Moreover, the acceptance e.g. of dual-purpose chicken and the actual willingness to pay at the point-of-sale as well as the sensory evaluation are open questions.

With regard to RQ3, we conclude that consumers react differently to increasing prices for meat. While the majority would not change their purchase behavior, around one-third would reduce their meat consumption. Only a very small share of the sample would increase their meat consumption due to higher perceived animal welfare standards. Referring to current regulations in Germany, the study highlights the potential for a stronger regulatory framework from the citizen perspective. Consumers advocate that chicken have an adequate space to live, i.e. a low stocking density and an adequate housing system. Moreover, the regulator should solve the issue of killing day-old chicks, in particular by focusing on breeding for mast and laying, and include regulations regarding bill shortening and the housing system, in particular with access to different climate zones.

Although we tried to reduce the hypothetical character of this study, one has to keep in mind that a gap between the hypothetical and actual WTP for higher animal welfare standards might exist. Analyzing actual purchase behavior using scanner data that includes specific product details like animal welfare labels (which is often lacking in current data sources) would allow investigating this issue in further detail. Moreover, other potentially important animal husbandry parameters could be included in future analyses. The complexity of the choice experiment might have resulted in information overload 
for respondents. However, we could not identify systematic refusal to consider the options in the choice set within our sample. Despite the serious consideration by respondents, the estimates may be impacted by limited scope sensitivity (Goldberg and Roosen 2007), an issue that could be investigated in futures studies.

This study provides insights for legal revisions for adequate animal welfare standards from a citizen perspective, while other aspects such as the view of the farmers, cost issues and the implementation of new regulations also need to be considered. Future studies could conduct cost-benefit analyses of higher welfare standards enabling a more complete assessment of alternative options.

Acknowledgment The authors thank Johanna Dahlhausen for her help in planning this study and the choice experiment.

\section{References}

Blaha T (2017) Wie sinnvoll sind Tierwohl-Label? J Consum Prot Food Saf 12:101-103. https://doi.org/ 10.1007/s00003-017-1105-1

Bundesministerium für Ernährung und Landwirtschaft (2016) Statistisches Jahrbuch über Ernährung, Landwirtschaft und Forsten - Jahrbuch 2016. Landwirtschaftsverlag GmbH, Münster

Carlsson F, Frykblom P, Lagerkvist CJ (2007) Consumer willingness to pay for farm animal welfare: mobile abattoirs versus transportation to slaughter. Eur Rev Agric Econ 34:321-344. https://doi.org/10.1093/ erae/jbm025

Clark B, Stewart GB, Panzone LA, et al. (2017) Citizens, consumers and farm animal welfare: a meta-analysis of willingness-to-pay studies. Food Policy 68:112-127. https://doi.org/10.1016/j.foodpol.2017.01. 006

de Jonge J, van der Lans IA, van Trijp HCM (2015) Different shades of grey: Compromise products to encourage animal friendly consumption. Food Qual Prefer 45:87-99. https://doi.org/10.1016/j. foodqual.2015.06.001

Goldberg I, Roosen J (2007) Scope insensitivity in health risk reduction studies: a comparison of choice experiments and the contingent valuation method for valuing safer food. J Risk Uncertain 34:123-144. https://doi.org/10.1007/s11166-007-90069

Hanley N, Shogren J, White B (2001) Introduction to environmental economics. Oxford University Press, Oxford

Hausman J, McFadden D (1984) Specification tests for the multinomial logit model. Econometrica 52:1219-1240. https://doi.org/10.2307/1910997

Heise H, Theuvsen L (2017) Die Mehrzahlungsbereitschaft für Milch, Eier und Fleisch aus Tierwohlprogrammen: Eine repräsentative Verbraucherbefragung. J Consum Prot Food Saf 12:105-113. https://doi.org/10.1007/s00003-016-10620

Hole AR, Kolstad JR (2012) Mixed logit estimation of willingness to pay distributions: a comparison of models in preference and WTP space using data from a health-related choice experiment. Empir Econ 42:445-469. https://doi.org/10.1007/s00181011-0500-1

Karl H, Noleppa S (2017) Kosten europäischer Umweltstandards und von zusätzlichen Auflagen in der deutschen Landwirtschaft - Eine Analyse und Hochrechnung für durchschnittliche Betriebe und den Sektor. HFFA Research Paper, Berlin.

Lagerkvist CJ, Hess S (2011) A meta-analysis of consumer willingness to pay for farm animal welfare. Eur Rev Agric Econ 38:55-78. https://doi.org/10. 1093/erae/jbq043

Lusk JL, Roosen J, Bieberstein A (2014) Consumer acceptance of new food technologies: causes and roots of controversies. Annu Rev Resour Econ 6:381-405.

McFadden D (1974) Conditional logit analysis of qualitative choice behavior. In: Zarembka P (ed) Frontiers in Econometrics. Academic Press, New York, pp 105-142

Norwood FB, Lusk JL (2011) Compassion, by the Pound: The Economics of Farm Animal Welfare. Oxford University Press, Oxford

Scientific Advisory Board on Agricultural Policy (2015) Pathways to a socially accepted livestock husbandry in Germany - Executive Summary and Synthesis Report Scientific. Berlin 
Smith ME, Pinckaers M (2012) Netherlands - Retail Foods - The Benelux Food Retail Market. The Hague

Statistisches Bundesamt (2011) Wirtschaftsrechnungen Einkommens- und Verbrauchsstichprobe Aufwendungen privater Haushalte für Nahrungsmittel, Getränke und Tabakwaren 2008. Wiesbaden Street DJ, Burgess L, Louviere JJ (2005) Quick and easy choice sets: Constructing optimal and nearly optimal stated choice experiments. Int J Res Mark 22:459-470. https://doi.org/10.1016/j.ijresmar.2005. 09.003

Zander K, Isermeyer F, Bürgelt D et al. (2013) Erwartungen der Gesellschaft an die Landwirtschaft. Braunschweig 


\section{Bürgerreaktionen auf Zielkonflikte in der Hühnchenmast}

Winnie Isabel Sonntag ${ }^{1}$, Stefan Golze ${ }^{1}$, Arne Kutschbach $^{1}$, Birgit Gassler ${ }^{1}$, Achim Spiller ${ }^{1}$

${ }^{1}$ Georg-August-Universität Göttingen, Department für Agrarökonomie und Rurale Entwicklung Marketing für Lebensmittel und Agrarprodukte winnie.sonntag@agr.uni-goettingen.de

\section{Einleitung}

Eine steigende Besorgnis gegenüber intensiven Tierhaltungssystemen ist ein anhaltendes Phänomen in westlichen Gesellschaften (Van Loo et al. 2014). Insbesondere Produktionsformen mit großen Tierzahlen und ganzjähriger Stallhaltung, die einen hohen Mechanisierungsgrad aufweisen, wie bspw. die Masthühnchenhaltung, werden von der Gesellschaft negativ bewertet (Castellini et al. 2008). Einerseits werden Geflügelprodukte aufgrund ihres geringen Preises und ihres ernährungsphysiologischen Wertes gerne konsumiert, andererseits werden Produktsicherheit und hohe Tierwohl- und Umweltstandards erwartet (De Jonge und van Trijp 2013; Vanhonacker et al. 2010). Daraus entstehen zwangsläufig Zielkonflikte, die für Laien nicht ohne weiteres erkennbar sind (Zander et al. 2013).

Allgemein versteht man unter einem Zielkonflikt die Unvereinbarkeit zweier Ziele. Es kann kein Ziel realisiert werden, ohne ein anderes Ziel zu vernachlässigen (Laux et al. 2014). In Bezug auf die landwirtschaftliche Tierhaltung ist den meisten Bürgern der Zielkonflikt zwischen niedrigen Preisen und Tierwohl geläufig, allerdings existieren Zielkonflikte auch zwischen verschiedenen Nachhaltigkeitsdimensionen oder zwischen verschiedenen Tierschutzzielen (WBA 2015). Um eine höhere Akzeptanz der Tierhaltung zu erreichen, muss klar sein, welche Ziele von Bürgern im Konfliktfall präferiert werden.

Bisher ist allerdings kaum bekannt, wie Bürger reagieren und sich entscheiden, wenn sie sich mit typischen Zielkonflikten in der Hühnchenhaltung (wie bspw. höherer Stickstoffeintrag vs. Tageslicht bei der Auslaufhaltung) auseinandersetzen müssen. Vor diesem Hintergrund wurden zunächst in einem innovativen Mixed-Methods-Ansatz 10 intensive Einzelinterviews mit deutschen Bürgern geführt und diese Ergebnisse anschließend in einer quantitativen Befragung mit 303 Probanden verifiziert. In beiden Studien wurden neutral gehaltene Informationstexte und Bilder eingesetzt, um den Teilnehmern ausgewählte Zielkonflikte zu veranschaulichen. Es wurde ermittelt, wie die Probanden reagieren, wenn sie mit Nachteilen eines positiv wahrgenommenen Systems und mit Vorteilen eines negativ wahrgenommenen Systems konfrontiert werden. Die Studie lässt erste Schlussfolgerungen darüber zu, wie Bürger verschiedene Nachhaltigkeitsziele gegeneinander abwägen und welche Priorisierung sie vornehmen. Die Ergebnisse unterstützen politische Entscheider und die Praxis bei der Entwicklung innovativer Tierhaltungssysteme unter Einbeziehung von Bürgermeinungen.

\section{Vorgehensweise und Methoden}

Vor dem Hintergrund der skizzierten Forschungslücke wurde ein qualitatives, exploratives Vorgehen in Form von Einzelinterviews mit einem quantitativen Vorgehen in Form einer Online-Befragung kombiniert.

\subsection{Qualitative Interviews}

Für die qualitativen Interviews wurden Bürger über Anzeigen in der Presse und sozialen Medien rekrutiert. Zwischen dem 31. Oktober und dem 03. November 2016 wurden zehn 90-minütige halbstrukturierte, intensive Leitfadeninterviews mit Bürgern geführt, die keine Vorkenntnisse oder berufliche Erfahrungen in der Landwirtschaft hatten. Die fünf Frauen waren durchschnittlich 41 Jahre alt und die fünf Männer im Durchschnitt 35 Jahre alt. Unterstützt von Bildern und neutral formulierten Texten wurden Zielkonflikte bei der Auslaufhaltung für Masthühnchen und der Bodenhaltung auf Einstreu dargestellt (Abb. 1 und 2). Die Vor- und Nachteile wurden anhand einer Literaturrecherche identifiziert und von Experten auf Neutralität begutachtet (Tab. 1). Das Vorgehen war in allen Interviews identisch. Die Probanden wurden nach einer Einführungsphase und der Frage nach Konsumgewohnheiten zunächst gebeten, die Bilder der Auslaufhaltung und der Bodenhaltung ohne Informationen zu beurteilen und ihre Wahrnehmungen zu schildern. Danach erhielten sie die Auflistung der Vor- und Nachteile beider Systeme. Die Interviews wurden aufgezeichnet, anschließend transkribiert und inhaltsanalytisch nach Mayring (2010) ausgewertet. Dieser Auswertungsmethode folgend, wurden Aussagen zunächst paraphrasiert, generalisiert und dadurch deduktive Hauptkategorien gebildet (Mayring 2010). Anschließend wurden induktive Kategorien abgeleitet und das 
Kategoriensystem weiter verfeinert (Kuckarzt 2016). Somit konnten die Aussagen den gebildeten Kategorien zugeordnet werden. Die Datenanalyse erfolgt mittels der Software MAXQDA.

\subsection{Quantitative Online-Befragung}

In Anlehnung an die Ergebnisse der qualitativen Interviews wurde im März 2017 eine deutschlandweite Online-Umfrage mit Quoten für Alter, Geschlecht und Bildung in Zusammenarbeit mit einem Panel-Anbieter durchgeführt. Insgesamt wurden 303 deutsche Bürger über 18 Jahre befragt. Der Fragebogen gliederte sich in 4 Blöcke:

1. Allgemeine soziodemographische Angaben,

2. Nähe zur Landwirtschaft und Konsumverhalten,

3. Einstellungsfragen zum Informations- und Einkaufsverhalten sowie zur Tierwohldiskussion und
4. Bewertung der identifizierten Zielkonflikte. In der quantitativen Befragung wurden die Reduzierung der Besatzdichte sowie die Auslaufhaltung für Masthühnchen als Zielkonflikte thematisiert.

Im vierten Block war das Vorgehen für jeden Zielkonflikt identisch. Zunächst erhielten die Probanden gleichzeitig Bilder und Informationstexte mit Vor- und Nachteilen des jeweiligen Systems (Abb. 1 bis 2 und Tab. 1). Nach der Informationsgabe wurden die Teilnehmer gebeten, einzelne Zielkonflikte zum jeweiligen System zu bewerten. Ziel war herauszufinden, wie sich die Teilnehmer bei Konflikten zwischen verschiedenen Nachhaltigkeitszielen (wie z.B. Tierwohl vs. Umweltschutz) und zwischen altruistischen und egoistischen Zielen (wie z.B. Tierwohl vs. Produktqualität) entscheiden. Die Auswertung der erhobenen Daten wurde mittels IBM SPSS 24 durchgeführt. 


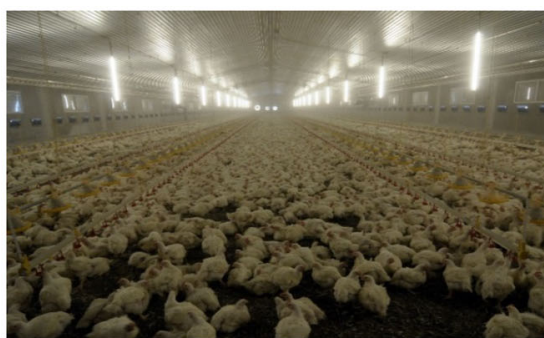

Bild A

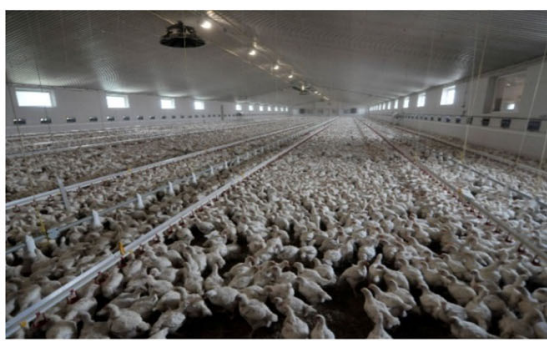

Bild C

Quelle: (C Christian Mühlhausen, Agentur Landpixel; Die Bilder B und C wurden ebenfalls bei der quantitativen Online-Befragung verwendet.

Abb. 1 Eingesetzte Bilder zum Zielkonflikt Bodenhaltung auf Einstreu

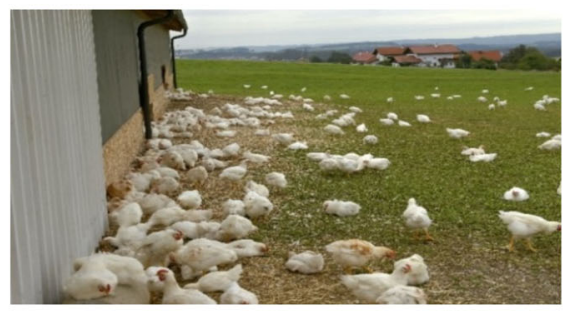

Bild A

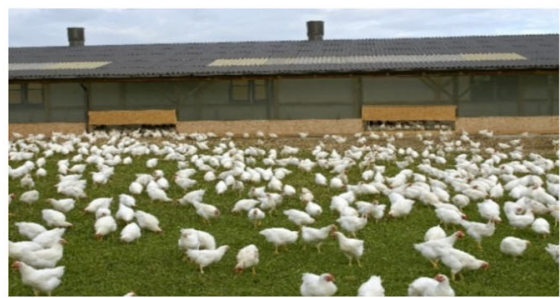

Bild C

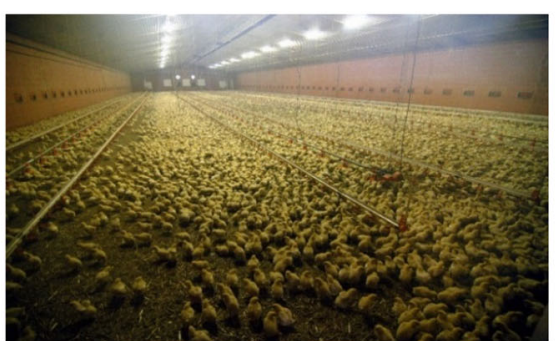

Bild B

Quelle: (C) agrarfoto.com; Die Bilder A und C wurden ebenfalls bei der quantitativen OnlineBefragung verwendet.

Abb. 2 Eingesetzte Bilder zum Zielkonflikt Auslaufhaltung 
Tab. 1 Informationen für die Interviewteilnehmer

\begin{tabular}{|c|c|c|c|}
\hline & & Vorteile & Nachteile \\
\hline \multirow[t]{6}{*}{ Bodenhaltung } & 1 & $\begin{array}{l}\text { Umweltparameter wie Temperatur, Licht und } \\
\text { Luftwechselrate können kontrolliert werden }\end{array}$ & $\begin{array}{l}\text { Aufgrund der hohen Tierzahlen kommt es bei den } \\
\text { Hähnchen zu Behinderungen beim Aufstehen und } \\
\text { Bewegen }\end{array}$ \\
\hline & 2 & Emissionen wie Geruch oder Lärm sind verringert & $\begin{array}{l}\text { Dies hat eine Bewegungseinschränkung für die } \\
\text { Hähnchen zur Folge }\end{array}$ \\
\hline & 3 & $\begin{array}{l}\text { Durch Stallhaltung wird die physiologische Entwicklung } \\
\text { der Tiere optimiert. Sie ist ökonomisch sehr effizient und } \\
\text { weniger arbeitsintensiv }\end{array}$ & Das Ausüben natürlicher Verhaltensweisen ist erschwert \\
\hline & 4 & $\begin{array}{l}\text { Hygienische Probleme durch Krankheitsüberträger aus } \\
\text { der Umwelt sind minimiert }\end{array}$ & $\begin{array}{l}\text { Die Tiere weisen mehr Befiederungsdefekte durch } \\
\text { Kratzverletzungen auf }\end{array}$ \\
\hline & 5 & $\begin{array}{l}\text { Erhöhte Tiersicherheit, da die Tiere nicht mit } \\
\text { Beutegreifern in Kontakt kommen }\end{array}$ & $\begin{array}{l}\text { Kotplatz und Liegebereich können aufgrund der } \\
\text { Besatzdichte weniger getrennt werden }\end{array}$ \\
\hline & 6 & $\begin{array}{l}\text { Durch Intensivhaltung großer Tierzahlen verringert sich } \\
\text { der Flächenbedarf pro Tier }\end{array}$ & $\begin{array}{l}\text { Die Gefahr von Infektionen durch Ausscheidungen ist } \\
\text { vergrößert }\end{array}$ \\
\hline \multirow[t]{6}{*}{ Auslaufhaltung } & 1 & $\begin{array}{l}\text { Es wird den Tieren ermöglicht in Interaktion mit der } \\
\text { Umwelt zu treten. Die Hähnchen haben Wahlfreiheit } \\
\text { zwischen verschiedenen Klimabedingungen und } \\
\text { Zugang zum Tageslicht }\end{array}$ & $\begin{array}{l}\text { Mit diesem System sind erhöhte Investitions- und } \\
\text { Betriebskosten verbunden }\end{array}$ \\
\hline & 2 & $\begin{array}{l}\text { Sozialverhalten und arttypisches Verhalten wie scharren, } \\
\text { picken oder sandbaden können besser ausgelebt } \\
\text { werden }\end{array}$ & $\begin{array}{l}\text { Durch den höheren Platzbedarf und die erweiterten } \\
\text { Bewegungsmöglichkeiten der Tiere, die zu einem } \\
\text { erhöhten Futterverbrauch führen, ist die Haltung } \\
\text { kostenintensiver }\end{array}$ \\
\hline & 3 & $\begin{array}{l}\text { Die Tiere weisen weniger Befiederungsdefekte durch } \\
\text { Kratz- und Pickverletzungen auf }\end{array}$ & $\begin{array}{l}\text { Aufgrund des Kontakts zur Außenwelt und Wildvögeln ist } \\
\text { der Hygienestatus schwerer aufrecht zu halten, da eine } \\
\text { erhöhte Gefahr der Aufnahme von Krankheitserregern } \\
\text { besteht }\end{array}$ \\
\hline & 4 & $\begin{array}{l}\text { Die Tiere haben weniger schwere Läsionen an den } \\
\text { Fußballen }\end{array}$ & $\begin{array}{l}\text { Der Kontakt zu Beutegreifern verringert die } \\
\text { Tiersicherheit }\end{array}$ \\
\hline & 5 & Es steht mehr Platz pro Tier zur Verfügung & $\begin{array}{l}\text { Es kommt zu einer Übernutzung des stallnahen } \\
\text { Bereiches, indem die Grasnarbe zerstört wird und starke } \\
\text { Überdüngung stattfindet }\end{array}$ \\
\hline & 6 & Die Tiere leben in der Regel länger & Insgesamt erweist sich dieses System als arbeitsintensiver \\
\hline
\end{tabular}

Quelle: Bessei 2006; Dawkins et al. 2003; Ellendorff et al. 2002; Fanatico et al. 2005; Feddes et al. 2002; Ghosh et al. 2012; Knierim 2013; Puron et al. 1995; Reiter und Bessei 2000; Stahl et al. 2002; Tuyttens et al. 2008; Wolf-Reuter 2004

\section{Ergebnisse}

\subsection{Qualitative Interviews}

\subsubsection{Einschätzung des eigenen Wissensstandes und Konsumverhaltens}

Das Vorwissen und die Erfahrungen zum Thema Hühnchenmast waren bei den Probanden eher gering. Direkten Kontakt zur Hühnchenmast hatten lediglich 2 der Befragten. Auf die Frage, wie sich die Interviewten aktuelle Haltungsformen in der Hühnchenmast vorstellen, wurden fast ausschließlich negative Begriffe verwendet. Unter den Probanden befanden sich eine Veganerin, ein Vegetarier und acht Fleischesser. Der Fleischkonsum der Fleischesser schwankte von einer Fleischmahlzeit pro Woche bis hin zum täglichen Fleischkonsum. Sieben Probanden aßen auch Hühnchenfleisch.

\subsubsection{Konfrontation mit Zielkonflikten}

Nur drei Probanden konnten auf die Frage, was unter einem Zielkonflikt zu verstehen sei, eine konkrete Antwort geben. Die restlichen Teilnehmer erklärten, nicht zu wissen, was ein Zielkonflikt sei. Zur Unterstützung wurde deshalb ein thematisch entferntes Beispiel genannt (Geldanlage mit niedrigem Risiko aber auch geringer Rendite). Anschließend wurde offen abgefragt, welche Zielkonflikte in der Haltung von Masthühnchen vorliegen könnten. Neun von zehn Probanden konnten mindestens einen Zielkonflikt nennen. Die häufigsten Beispiele waren der Konflikt zwischen Produktpreis bzw. Zahlungsbereitschaft und Qualität der Tierhaltung bzw. des Fleisches. 


\section{Bodenhaltung auf Einstreu}

Beim Fallbeispiel Bodenhaltung auf Einstreu wurden die Probanden zunächst gebeten Bilder (Abb. 1 und 2) ohne Informationen zu bewerten. Insgesamt beschrieben die Teilnehmer ihre Wahrnehmungen als negativ. Ein Großteil der Befragten äußerte Besorgnis über das Wohl der Tiere in dieser Haltungsform. Vor allem, dass die Tiere zumindest Tageslicht sehen, wurde als Wunsch geäußert. Anschließend erhielten die Probanden Texte mit Vorund Nachteilen (Zielkonflikten) des jeweiligen Haltungssystems. Sie wurden erneut gebeten ihre Wahrnehmungen zu beschreiben und sich für eine Argumentationsrichtung, also entweder für die Voroder die Nachteile, zu entscheiden. Während die Nachteile allen Befragten fast ausnahmslos bekannt waren, erschienen die Vorteile überwiegend neu. Die Probanden äußerten größtenteils Verständnis für die Tierhalter und konnten die genannten Vorteile des Systems nachvollziehen. Dieses Verständnis wollten die Probanden jedoch nicht als Akzeptanz verstanden wissen. $\mathrm{Zu}$ den plausiblen Argumenten zählte in erster Linie die erhöhte Kontrolle der Umweltparameter wie Temperatur, Licht und Luftwechselrate. Mehrmals genannt wurde auch die ökonomische Effizienz, wobei dies als positiv für die Produzenten und nicht für das Tier oder die Verbraucher gewertet wurde. Dass mit dem geschlossenen System eine Minimierung hygienischer Probleme durch Krankheitsüberträger aus der Umwelt einhergeht, wurde als vorteilhaft empfunden. Als nachteilig führten die Probanden das unnatürliche und nicht arttypische Verhalten der Hühnchen in einem solchen System an. Insgesamt bewertete eine Probandin abschließend die Bodenhaltung als positiv, die anderen 9 Teilnehmer gewichteten die Nachteile der Bodenhaltung stärker. Die zur Verfügung gestellte Information führte nach eigener Angabe bei keinem Probanden zu einer veränderten Wahrnehmung der Bodenhaltung auf Einstreu.

\section{Auslaufhaltung für Masthühnchen}

Nachfolgend wurden die Probanden mit Bildern und Zielkonflikten der Auslaufhaltung konfrontiert. Die Bilder der Auslaufhaltung wurden sehr positiv bewertet. Vereinzelt schlossen einige Probanden aufgrund der Auslaufhaltung auch auf ein hochwertigeres Nahrungsmittel. Ein weiterer, oft wiedergegebener Vorteil war, dass sich die Tiere durch die Interaktion mit der Umwelt arttypischer verhalten könnten. Auf der Seite der Nachteile wurde die Problematik der erhöhten Betriebskosten und des Arbeitsaufwandes verdeutlicht, für die persönliche Beurteilung dieses Haltungssystems war dies aber nicht ausschlaggebend. Die erschwerte Aufrechterhaltung des Hygienestatus wurde negativ bewertet und weckte bei den Probanden die Sorge, dass die Tiere mehr Antibiotika bekommen, sich dadurch die Fleischqualität verschlechtert und somit auch die eigene Gesundheit negativ beeinflusst wird. Insgesamt gaben die Probanden aber an, dass sich ihr positiver Eindruck des Haltungssystems durch die zur Verfügung gestellte Information noch verstärkt habe und dass es anzustreben sei, deutlich mehr Tiere in diesem System zu halten.

\section{Quantitative Befragung}

Auf Basis der Ergebnisse der Interviews wurde eine Online-Befragung mit 303 Teilnehmenden durchgeführt. Es nahmen insgesamt 48,8 \% männliche und $51,2 \%$ weibliche Teilnehmende an der Online-Befragung teil. Diese waren im Mittel 48,6 Jahre alt und größtenteils Angestellte (35,3\%) und Rentner (26,1\%), die oftmals in Zwei-Personen-Haushalten (43,6 \%) oder in Single-Haushalten $(29,0 \%)$ lebten. Insgesamt spiegelt die Stichprobe die deutsche Bevölkerung relativ gut wider.

Einschätzung des eigenen Wissensstandes und Konsumverhaltens

Bei der Frage nach ihrem Bezug zur Masthühnchenhaltung schätzten die Probanden ihr Interesse an der Landwirtschaft (73,6 \%) und an der Hühnchenhaltung (76,5 \%) als mittel bis sehr hoch ein. Das eigene Wissen über die Hühnchenhaltung wurde hingegen als mittel bis gering bewertet (75,2\%). 93,7 \% der Probanden kaufen Fleisch und Wurstwaren und rund $80 \%$ der Befragten essen mehrfach pro Woche bis täglich Fleisch.

\section{Konfrontation mit Zielkonflikten}

Nachdem die Probanden Bilder und Informationstexte zu den jeweiligen Haltungsformen erhalten hatten (s. Tab. 1 und Abb. 1 und 2 im Anhang), wurden sie gebeten, Zielkonflikte anhand einer 5-stufigen Skala (von „stimme voll und ganz zu“ bis „stimme ganz und gar nicht zu“) zu bewerten.

Reduzierung der Besatzdichte

Bei den Zielkonflikten zur Reduzierung der Besatzdichte entschied sich der Großteil der Teilnehmer für das Wohl der Tiere (s. Tab. 2). Weder ein geringer Preis noch eine bessere wirtschaftliche Situation des Landwirts oder eine gleichbleibende Produktqualität konnten für die Probanden Einschränkungen des Tierwohls rechtfertigen. Lediglich ein guter Gesundheitszustand der Tiere, bedingt durch moderne Stalltechnik und optimales Stallmanagement, führte $\mathrm{zu}$ etwas Unsicherheit in der Bewertung. 
Tab. 2 Bewertung der Zielkonflikte zur Reduzierung der Besatzdichte

\begin{tabular}{llll}
\hline Zielkonflikt & Stimme voll & Teils/teils & Stimme nicht zu \\
Reduzierung der & und ganz zu & \& stimme ganz \\
Besatzdichte & \& stimme zu & und gar nicht zu \\
\hline
\end{tabular}

Es ist akzeptabel, dass die Tiere weniger Platz haben, wenn ...

der Fleischpreis $\quad 9 \% \quad 18 \% \quad 73 \%$

gering bleibt

die Landwirte

$14 \%$

$31 \%$

$55 \%$

kostendeckend

arbeiten können

gleich bleibt

die Tiere durch den $35 \% \quad 28 \% \quad 37 \%$

Einsatz von

moderner

Stalltechnik und

gutem

Stallmanagement

gesund sind

Es ist akzeptabel, dass die Tiere unter erhöhten Stresslevels leben, wenn ...

der Fleischpreis

gering bleibt

$8 \% \quad 16 \% \quad 76 \%$

die Landwirte

$12 \%$

$21 \% \quad 67 \%$

kostendeckend

arbeiten können

die Fleischqualität $\quad 12 \% \quad 21 \% \quad 67 \%$

gleich bleibt

die Tiere durch den $22 \% \quad 23 \% \quad 54 \%$

Einsatz von

moderner

Stalltechnik und

gutem

Stallmanagement

gesund sind

Es ist akzeptabel, dass die Tiere zum Ende der Mastperiode auf feuchter Einstreu leben, wenn ...

\begin{tabular}{lccc}
$\begin{array}{l}\text { der Fleischpreis } \\
\text { gering bleibt }\end{array}$ & $5 \%$ & $17 \%$ & $78 \%$ \\
$\begin{array}{l}\text { die Landwirte } \\
\text { kostendeckend } \\
\text { arbeiten können } \\
\text { die Fleischqualität } \\
\text { gleich bleibt }\end{array}$ & $8 \%$ & $22 \%$ & $70 \%$ \\
$\begin{array}{l}\text { die Tiere durch den } \\
\text { Einsatz von }\end{array}$ & $17 \%$ & $22 \%$ & $68 \%$ \\
moderner & & $26 \%$ & $57 \%$ \\
$\begin{array}{l}\text { Stalltechnik und } \\
\text { gutem }\end{array}$ & & & \\
$\begin{array}{l}\text { Stallmanagement } \\
\text { gesund sind }\end{array}$ & & & \\
\hline
\end{tabular}

Quelle: Eigenen Erhebung, $n=303$

\section{Auslaufhaltung}

Ähnliche Resultate fanden sich bei der Bewertung der Zielkonflikte der Auslaufhaltung (s. Tab. 3). So nahm der Großteil der Probanden negative Auswirkungen der Auslaufhaltung auf die Umwelt und eine Erhöhung des Preises in Kauf, wenn es den Tieren im Gegenzug besser geht. Eine erhöhte Infektionsgefahr durch pathogene Erreger und Keime wurde als Zielkonflikt noch am stärksten gewichtet, trotzdem überwogen die Präferenzen für die Auslaufhaltung.

Tab. 3 Bewertung der Zielkonflikte zur Auslaufhaltung

\begin{tabular}{|c|c|c|c|}
\hline $\begin{array}{l}\text { Zielkonflikt } \\
\text { Auslaufhaltung }\end{array}$ & $\begin{array}{l}\text { Stimme } \\
\text { voll und } \\
\text { ganz zu \& } \\
\text { stimme zu }\end{array}$ & Teils/teils & $\begin{array}{l}\text { Stimme nicht } \\
\text { zu \& stimme } \\
\text { ganz und gar } \\
\text { nicht zu }\end{array}$ \\
\hline
\end{tabular}

Ein gesteigerter Nährstoffeintrag (Nitrat) in den Boden ist akzeptabel, wenn ...

$\begin{array}{lccc}\begin{array}{l}\text { die Tiere Zugang } \\ \text { zu Tageslicht und } \\ \text { frischer Luft } \\ \text { haben }\end{array} & 51 \% & 30 \% & 19 \% \\ \begin{array}{l}\text { die Tiere ihre } \\ \text { arttypischen } \\ \begin{array}{l}\text { Verhaltensmuster } \\ \text { ausüben können }\end{array}\end{array} & 61 \% & 25 \% & 14 \% \\ \begin{array}{l}\text { die Tiere sich } \\ \text { besser } \\ \text { fortbewegen }\end{array} & 63 \% & 24 \% & 13 \% \\ & & & \\ \end{array}$

Eine höhere Gefahr der Infektion mit Krankheiten ist akzeptabel, wenn ...

die Tiere Zugang $\quad 46 \% \quad 34 \% \quad 20 \%$

zu Tageslicht und

frischer Luft

haben

die Tiere ihre $\quad 52 \% \quad 29 \% \quad 19 \%$

arttypischen

Verhaltensmuster

ausüben können

die Tiere sich $\quad 55 \% \quad 27 \% \quad 18 \%$

besser

fortbewegen

Eine höhere Gefahr durch Angriffe von Raubtieren ist akzeptabel, wenn ...

$\begin{array}{llcc}\begin{array}{l}\text { die Tiere Zugang } \\ \text { zu Tageslicht und } \\ \text { frischer Luft } \\ \text { haben }\end{array} & 61 \% & 27 \% & 12 \% \\ \begin{array}{l}\text { die Tiere ihre } \\ \text { arttypischen }\end{array} & 65 \% & 26 \% & 9 \% \\ \begin{array}{l}\text { Verhaltensmuster } \\ \text { ausüben können } \\ \text { die Tiere sich } \\ \text { besser } \\ \text { fortbewegen }\end{array} & 66 \% & 25 \% & 9 \% \\ \end{array}$


Tab. 3 continued

\begin{tabular}{|c|c|c|c|}
\hline $\begin{array}{l}\text { Zielkonflikt } \\
\text { Auslaufhaltung }\end{array}$ & $\begin{array}{l}\text { Stimme voll } \\
\text { und ganz zu } \\
\& \text { stimme zu }\end{array}$ & Teils/teils & $\begin{array}{l}\text { Stimme nicht zu } \\
\text { \& stimme ganz } \\
\text { und gar nicht zu }\end{array}$ \\
\hline \multicolumn{4}{|c|}{ Ein höherer Preis für Hähnchenfleisch ist gerechtfertigt, wenn .. } \\
\hline $\begin{array}{l}\text { die Tiere Zugang zu } \\
\text { Tageslicht und } \\
\text { frischer Luft haben }\end{array}$ & $71 \%$ & $20 \%$ & $9 \%$ \\
\hline $\begin{array}{l}\text { die Tiere ihre } \\
\text { arttypischen } \\
\text { Verhaltensmuster } \\
\text { ausüben können }\end{array}$ & $73 \%$ & $18 \%$ & $9 \%$ \\
\hline $\begin{array}{l}\text { die Tiere sich besser } \\
\text { fortbewegen }\end{array}$ & $75 \%$ & $17 \%$ & $8 \%$ \\
\hline \multicolumn{4}{|c|}{ Trockeneres Geflügelfleisch ist gerechtfertigt, wenn ... } \\
\hline $\begin{array}{l}\text { die Tiere Zugang zu } \\
\text { Tageslicht und } \\
\text { frischer Luft haben }\end{array}$ & $56 \%$ & $29 \%$ & $15 \%$ \\
\hline $\begin{array}{l}\text { die Tiere ihre } \\
\text { arttypischen } \\
\text { Verhaltensmuster } \\
\text { ausüben können }\end{array}$ & $58 \%$ & $27 \%$ & $15 \%$ \\
\hline $\begin{array}{l}\text { die Tiere sich besser } \\
\text { fortbewegen }\end{array}$ & $59 \%$ & $26 \%$ & $15 \%$ \\
\hline
\end{tabular}

Quelle: Eigenen Erhebung, $\mathrm{n}=303$

\section{Schlussfolgerungen}

Bislang wurde der Umgang von Bürgern mit Zielkonflikten in der Tierhaltung lediglich als Nebenbefund thematisiert (Zander et al. 2013). Der innovative Zugang eines Mixed-Methods-Ansatzes, der die Bürgerwahrnehmung und -bewertung der Masthühnchenhaltung sowohl qualitativ als auch quantitativ detailliert darstellt, liefert daher wertvolle Hinweise für nachhaltige Veränderungsprozesse in der Tierhaltung. Insbesondere in Kombination mit den Ergebnissen von Rovers et al. 2017 (in diesem Heft) wird so ein breiteres Verständnis von Bürgererwartungen und -wahrnehmungen geschaffen. Die explorativen Ergebnisse zeigen, dass ein Bewusstsein für Zielkonflikte in der Tierhaltung im Allgemeinen und in der Hühnchenmast im Besonderen bei Bürgern kaum bis gar nicht vorhanden ist. Den Befragten ist meist nicht bekannt, dass die mehrheitlich präferierten Haltungssysteme auch Nachteile aufweisen. Entsprechend wurden die Probanden im zweiten Schritt mit balancierten Informationen (gleich vielen Vor- wie Nachteilen) zu unterschiedlichen Haltungssystemen konfrontiert.

Bei der Analyse der Bewertung vorgegebener Zielkonflikte zeigte sich, dass das Wohl der Tiere dominiert. So war ein hoher Tierwohlstatus von größerer Bedeutung als egoistische Ziele, wie bspw. ein geringer Verbraucherpreis oder die Produktqualität. In gleichem Maße entschieden sich Bürger für das Wohl der Tiere, auch zu Lasten von anderen Nachhaltigkeitszielen wie dem Umweltschutz. Bei Zielkonflikten innerhalb des Tierschutzes wurde das Ausleben von natürlichen Verhaltensmustern höher gewichtet als die Tiergesundheit, hier war die Abwägung allerdings weniger eindeutig. Wirtschaftlichkeitsgesichtspunkte für die Landwirte zählten im Konfliktfall besonders wenig. Zudem zeigte sich in den Interviews, dass Probanden von einer schlechten Haltung teilweise auch auf eine nachteilige Produktqualität schließen und Gefahren für die eigene Gesundheit ableiten.

Auch wenn die quantitative Studie aufgrund der mit rund 300 Probanden begrenzten Stichprobe nicht repräsentativ für die deutsche Bevölkerung ist, so lassen sich dennoch vorsichtige Schlussfolgerungen ableiten. Insgesamt verbesserte sich trotz ausgeglichener, neutraler Information durch Bilder und Texte die Wahrnehmung von als negativ empfundenen Systemen (Bodenhaltung auf Einstreu, hohe Besatzdichte) nicht. Auch führte die Konfrontation mit Nachteilen der als positiv empfundenen Auslaufhaltung nicht dazu, dass Probanden diese Haltungsform kritisch sahen.

Daraus lässt sich ableiten, dass eine bürgernahe Kommunikationsstrategie, die auf Aufklärung und Informationen setzt, wenig erfolgreich sein wird, Systeme mit negativem Image positiv darzustellen. Sehen sich Bürger mit Zielkonflikten konfrontiert, dominiert das Tierwohl. Das deutet darauf hin, dass Haltungssysteme, die Tierwohlaspekte nicht berücksichtigen, langfristig schwer zu vermitteln sein werden. In den qualitativen Interviews wurde immer wieder angesprochen, dass die Landwirtschaft die Zielkonflikte möglichst durch Innovationen auflösen müsste. Hieraus ergeben sich umfangreiche Forschungsdesiderate für die Nutztierwissenschaften.

\section{Literatur}

Bessei W (2006) Welfare of broilers - a review. Worlds Poult Sci J 62:455-466

Castellini C, Berri C, Le Bihan-Duval E, Martino G (2008) Qualitative attributes and consumer perception of organic and free-range poultry meat. Worlds Poult Sci J 64:500-512

Dawkins MS, Cook PA, Whittingham MJ, Mansell KA, Haroer AE (2003) What makes free-range broiler 
chickens range? In situ measurement of habitat preference. Anim Behav 66:151-160

De Jonge J, van Trijp HC (2013) Meeting heterogeneity in consumer demand for animal welfare: a reflection on existing knowledge and implications for the meat sector. J Agric Environ Ethics 26:629-661

Ellendorf F, Berk J, Dänicke S et al. (2002) Interdisziplinäre Bewertung unterschiedlich intensiver Produktionssysteme von Masthühnchen unter Aspekten von Tierschutz, Produktqualität, Umwelt, Wirtschaftlichkeit. Agrar-oder Verbraucherwende: Wer bestimmt den Markt?:20-41

Fanatico AC, Cavitt LC, Pillai PB, Emmert JL, Owens CM (2005) Evaluation of slower-growing broiler genotypes grown with and without outdoor access: meat quality. Poultry Sci 84:1785-1790

Feddes JJ, Emmanuel EJ, Zuidhof MJ (2002) Broiler performance, bodyweight variance, feed and water intake, and carcass quality at different stocking densities. Poultry Sci 81:774-779

Ghosh S, Majumber D, Goswami R (2012) Broiler performance at different stocking densities. Indian J Anim Res 46:381-384

Knierim U (2013) Auswirkungen der Besatzdichte in der Schwermast auf das Verhalten sowie die Fußund Beingesundheit von Masthühnern. Berl Munch Tierarztl Wochenschr 126:149-155

Kuckartz U (2016) Qualitative Inhaltsanalyse. Methoden, Praxis, Computerunterstützung. Beltz Juventa, Weinheim und Basel

Laux H, Gillenkirch RM, Schenk-Mathes HY (2014) Entscheidungstheorie. Springer, Berlin

Mayring P (2010). Qualitative Inhaltsanalyse. Grundlagen und Techniken. Beltz, Weinheim
Puron D, Santamaria R, Segura JC, Alamilla JL (1995) Broiler performance at different stocking densities. J Appl Poult Res 4:55-60

Reiter K, Bessei W (2000) Einfluß der Besatzdichte bei Broilern auf die Temperatur in der Einstreu und im Tierbereich. Arch Geflugelkd 64:204-206

Stahl P, Ruette S, Gros L (2002) Predation on freeranging poultry by mammalian and avian predators. Field loss estimates in a French rural area. Mammal Rev 32:227-234

Tuyttens F, Heyndrickx M, de Boeck M et al. (2008) Broiler chicken health, welfare and fluctuating asymmetry in organic versus conventional production systems. Livest Sci 113:123-132

Van Loo EJ, Caputo V, Nayga RM, Verbeke W (2014) Consumers' valuation of sustainability labels on meat. Food Policy 49:137-150

Vanhonacker F, Van Poucke E, Tuyttens F, Verbeke W (2010) Citizens' views on farm animal welfare and related information provision: Exploratory insights from flanders, Belgium. J Agric Environ Ethics 23:551-569

Wissenschaftlicher Beirat Agrarpolitik (WBA) (2015) Wege zu einer gesellschaftlich akzeptierten Nutztierhaltung. Kurzfassung des Gutachtens. Berlin

Wolf-Reuter M (2004) Bewertung unterschiedlich intensiver Produktionssysteme von Masthühnchen unter Berücksichtigung von Prozess- und Produktqualität. Dissertation, Tierärztliche Hochschule Hannover

Zander K, Isermeyer F, Bürgelt D, Christoph-Schulz I, Salamon P, Weible D (2013) Erwartungen der Gesellschaft an die Landwirtschaft. Abschlussbericht, Braunschweig 


\section{Mehr als eine Nische? Das Potenzial des Zweinutzungshuhns als Alternative zum Kükentöten}

\author{
Nanke Brümmer ${ }^{1}$, Jörg Luy $^{2}$, Anja Rovers ${ }^{1}$, Inken \\ Christoph-Schulz ${ }^{1}$ \\ ${ }^{1}$ Thünen-Institut für Marktanalyse, Braunschweig \\ ${ }^{2}$ Privates Forschungs- und Beratungsinstitut für \\ angewandte Ethik und Tierschutz INSTET gGmbH, \\ Berlin \\ nanke.bruemmer@thuenen.de
}

\section{Einleitung}

Durch das Gerichtsurteil des Oberverwaltungsgerichts Münster im Mai 2016 rückte das Töten männlicher Eintagsküken der Legehennenlinien in den Fokus der Öffentlichkeit. Das Gerichtsurteil besagt, dass das Töten der männlichen Eintagsküken aus wirtschaftlichen Gründen gerechtfertigt sei, da bisher noch keine praktikablen Alternativen zur Tötung vorliegen (Beckmann 2016). Zuvor war nicht vielen Bürgern bewusst, dass in Deutschland jährlich mehr als 45 Mio. männliche Eintagsküken von Legelinien getötet werden, was sich durch die mediale Berichterstattung schlagartig änderte.

Das Töten von Eintagsküken ist sowohl in der konventionellen als auch in der ökologischen Haltung von Legehennen üblich, weil die Mast der männlichen Küken der Legelinien aufgrund der geringen Mastleistung unrentabel ist (Damme 2015; Rautenschlein 2016). Die Geschlechtsbestimmung im Ei, die Mast der männlichen Tiere der Legehybriden oder Zweinutzungshuhnrassen bilden drei Alternativen zur Eintagskükentötung (Bruijnis et al. 2015). Als Zweinutzungshuhn werden Rassen bezeichnet, die sich sowohl für die Eier- als auch für die Fleischproduktion eignen. Züchter von Zweinutzungshühnern stehen allerdings vor dem Problem, dass sich schnelles Fleischwachstum und eine hohe Legeleistung schlecht miteinander vereinbaren lassen, denn die Hennen legen weniger und kleinere Eier und die Hähne setzen weniger Fleisch an und benötigen mehr Zeit und Futter für das Wachstum (Leenstra et al. 2011; Grashorn 2013). Folglich haben Eier und Fleisch von Zweinutzungshühnern andere Eigenschaften und sind teurer als Produkte von spezialisierten Hühnerrassen (Koenig et al. 2012; Kaufmann et al. 2016).

Die Konsumentenakzeptanz von Produkten der Zweinutzungshühner ist für weitere Anstrengungen von Züchtern, Landwirten und schließlich auch in
Bezug auf potenzielle Marketingstrategien für Fleisch und Eier von grundlegender Bedeutung. Das Ziel unserer Untersuchungen besteht daher darin, die Akzeptanzvoraussetzungen von Produkten der Zweinutzungshühner beim Verbraucher zu erfassen und zu verstehen. Dazu wurde eine Forschungskooperation mit dem von Prof. Dr. Silke Rautenschlein koordinierten Forschungsprojekt „IntegHof - Geflügelhaltung neu strukturiert ${ }^{*}$ der Tierärztlichen Hochschule Hannover initiiert. Im IntegHof-Projekt wird das Zweinutzungshuhn aus Sicht der Tiergesundheit, der Wirtschaftlichkeit, des Tierverhaltens sowie der Verbraucher untersucht.

\section{Methodik}

Um die komplexe und weitgehend unerforschte Thematik der Verbrauchersicht auf das Zweinutzungshuhn $\mathrm{zu}$ untersuchen, haben wir uns für eine qualitative Methode entschieden, die induktiv entwickelt wurde. Als Folge einer sich rasch verändernden Welt und aufkommender Herausforderungen gewinnen induktive Methoden an Bedeutung, weil sie besser als deduktive Ansätze auf Veränderungen im sozialen Kontext reagieren können (Flick 2009). Um die qualitativen Daten zu generieren, wurden Gruppendiskussionen durchgeführt. Gruppendiskussionen sind eine empirische Forschungsmethode mit Fokus auf Gruppendynamiken und Interaktionen zwischen den Teilnehmern (Finch und Lewis 2003). Laut Morgan (1997) sind Gruppendiskussionen eine Forschungsmethode, bei welcher Daten durch Gruppeninteraktionen zu einem vorgegebenen Thema gesammelt werden. Ziel ist es, eine Atmosphäre zu schaffen, die eine nahezu natürliche Konversation mit vielfältigen Meinungen und Aussagen fördert (Lamnek 2005). Darüber hinaus führt die Konversationssituation mit anderen Teilnehmern zu tieferen Einsichten in Motivationen und Rechtfertigungen und stimuliert neue Gedanken (Finch und Lewis 2003).

Die insgesamt sechs Gruppendiskussionen für die vorliegende Studie wurden im Juni 2016 mit jeweils sechs bis acht Teilnehmern in Berlin, München und Cloppenburg $^{13}$ durchgeführt. Die Teilnehmer wurden von einem Marktforschungsunternehmen rekrutiert und waren Konsumenten von Hühnerfleisch und Eiern. Personen mit einem beruflichen Hintergrund im Bereich Landwirtschaft, Lebensmittelindustrie oder Marktforschung wurden von den Diskussionen ausgeschlossen.

\footnotetext{
${ }^{13}$ Kreisstadt in einer Region mit intensiver Geflügelhaltung in Niedersachsen.
} 
Darüber hinaus wurden Quoten für das Alter (zwischen 20 und 70 Jahren), Geschlecht (Anteil der Männer und Frauen zwischen 33,3 \% und 66,6 \%) und Beschäftigung (Anteil der Beschäftigten ca. $67 \%$ ) berücksichtigt. Das genaue Thema war im Vorfeld nicht bekannt und während der Diskussionen wurden keine Informationen gegeben oder Fragen beantwortet. Der Leitfaden enthielt zuerst Fragen zum Eier- und Hühnchenfleischkonsum sowie zum Einkaufsverhalten. Anschließend wurden die Vorstellungen zur derzeitigen Legehennenund Masthühnchenhaltung erfasst und über die Eintagskükentötung sowie mögliche Alternativen zu dieser Praxis gesprochen. Anschließend wurde zum Konzept des Zweinutzungshuhns übergeleitet, Vor- und Nachteile diskutiert und wichtige Kaufkriterien für die Produkte erfasst. Abschließend wurde noch über das Thema einer möglichen Mehrzahlungsbereitschaft gesprochen. Die Diskussionen dauerten 90 Minuten und wurden audiound videogestützt aufgezeichnet und anschließend transkribiert. Die Transkripte wurden schließlich inhaltsanalytisch nach Mayring (2015) ausgewertet.

\section{Ergebnisse}

\subsection{Kaufkriterien für Hühnerfleisch und Eier sowie Konsumgewohnheiten}

Zu Beginn der Diskussionen wurden die Teilnehmer nach ihren Konsumgewohnheiten hinsichtlich Hühnerfleisch und Eiern gefragt. Die am häufigsten genannten Kaufkriterien bei Hühnerfleisch waren die Fleischfarbe, das Mindesthaltbarkeitsdatum und eine regionale Herkunft. Auch eine ökologische Tierhaltung war ein Aspekt, der mehrfach genannt wurde. Einige Teilnehmer erklärten, dass sie nicht in der Lage seien, sich Bio-Hühnerfleisch zu leisten und daher konventionell produziertes Hühnerfleisch kaufen würden. In diesem Zuge wurde auch erwähnt, dass die Haltungsbedingungen aufgrund der Angaben auf der Verpackung schwierig nachzuvollziehen seien. In Bezug auf die Konsumgewohnheiten wurde überdies deutlich, dass die Diskutanten Teilstücke wie Hühnerbrust oder -flügel gegenüber einem ganzen Huhn bevorzugen. Nur sehr wenige Diskussionsteilnehmer gaben an, gelegentlich ein ganzes Huhn zu kaufen und viele hatten noch nie ein ganzes Huhn zubereitet.

Im Hinblick auf den Kauf von Eiern gaben einige Diskussionsteilnehmer an, nicht auf das Haltungssystem zu achten. Für andere Diskutanten war dieser Aspekt sehr wichtig, sie würden vor allem Freilandoder Bio-Eier einkaufen. Ein weiterer Punkt war, dass einige Teilnehmer zwischen Eiern zum Kochen und Backen oder Frühstückseiern unterscheiden und daher bevorzugt Freiland- oder Bio-Eier als Frühstückseier verwenden würden, wobei das Haltungssystem bei Eiern für verarbeitete Lebensmittel als weniger bedeutend eingestuft wurde. Nur wenige Diskussionsteilnehmer erklärten, dass sie ausdrücklich weiße oder braune Eier wegen ihrer Assoziationen mit den Haltungsbedingungen der Hühner kaufen würden. Es wurde erwähnt, dass zu Ostern bevorzugt weiße Eier gekauft würden, um diese besser färben zu können. In Bezug auf die Ei-Größe waren die Meinungen vielfältiger. Einige Diskussionsteilnehmer erläuterten, dass sie nicht auf die Ei-Größe achten würden, wohingegen andere sagten, dass sie vorzugsweise große Eier auswählen würden. Zusammengefasst wurden das Haltungssystem, eine regionale Herkunft, das Mindesthaltbarkeitsdatum und die Unversehrtheit als wichtigste Kaufkriterien bei Eiern identifiziert.

\subsection{Allgemeine Wahrnehmungen der Hühnerhaltung}

Die spontanen Äußerungen der Teilnehmer zur Hühnerhaltung wurden von Begriffen wie "Tierfabriken", “Mangel an Transparenz" und "Profitgier” dominiert. In Bezug auf die Haltung der Legehennen waren die Diskussionsteilnehmer vor allem über die Qualität des Futters besorgt. Es wurde vermutet, dass die Hühner mit "Müll” gefüttert würden und sich dies in der Eiqualität widerspiegelt. In diesem Zusammenhang wurde auch das Kürzen der Schnäbel thematisiert. Das Bild von Hühnern in Käfigen, in welchen diese zusammengepfercht gehalten würden und keinen Platz für Bewegung hätten, war noch präsent, obwohl die konventionelle Käfighaltung seit 2010 in Deutschland verboten ist. Die Vorstellung der Haltung von Masthühnchen war von der Idee geprägt, dass diese den ganzen Tag fressen müssten, um an Gewicht zuzunehmen. Die Diskussionsteilnehmer vermuteten auch, dass es keine akzeptable Mensch-Tier-Beziehung geben würde und dass die Stallarbeiter die Tiere nicht angemessen behandeln würden. Die Vermutung der prophylaktischen Gabe von Antibiotika wurde von den Diskutanten wiederholt kritisch thematisiert. Zudem wurde überwiegend die Auffassung vertreten, dass Freilandhaltung am ehesten den Erwartungen an eine artgerechte Tierhaltung entspräche.

\subsection{Moralische Bedenken und Alternativen zum Kükentöten}

Das Thema des Tötens männlicher Eintagsküken wurde in jeder Gruppendiskussion von den 
Diskutanten ohne konkrete Nachfrage durch die Diskussionsleitung angesprochen. Den meisten Teilnehmern war diese gängige Praxis in der Legehennenhaltung bewusst. Dies könnte auch auf die Tatsache zurückzuführen sein, dass das Thema zu diesem Zeitpunkt in den Medien aufgrund des Gerichtsurteils zum Eintagskükentöten sehr präsent war. Viele Diskussionsteilnehmer drückten ihre Empörung über das Töten der Eintagsküken aus. Aussagen wie "Wenn man sich vorstellt, das sind Menschen. Die Jungen werden geschreddert und an Tiere verfüttert. Das ist ja ekelhaft” oder "Alle Männer bringt man um" demonstrieren, wie die moralische Urteilsbildung durch Einfühlung in die Betroffenen (Empathie) in der Praxis häufig abläuft. Viele Teilnehmer haben sich offenbar selbst in die Rolle der betroffenen Tiere versetzt und beurteilen das Eintagskükentöten nun aus dieser Perspektive. Die meisten Diskussionsteilnehmer waren sich einig, dass das Töten von Küken aus moralischer Sicht nicht akzeptabel sei und forderten, dass die Praxis gestoppt werden müsse. Nur wenige argumentierten, dass das Huhn sowieso getötet werden würde und es somit egal sei, ob früher oder später. Bei der Diskussion über die Gründe wurde davon ausgegangen, dass es aus „Profitgründen” sei und dass die Hähne nicht genug Fleischwachstum hätten. Ergänzend wurde gelegentlich geäußert, dass die Verbraucher diese Situation bislang nicht beeinflussen könnten.

Alle drei Alternativen zum Kükentöten waren nur wenigen Diskutanten bekannt. Die Geschlechtsbestimmung im Ei war eine Option, die mehrmals angesprochen wurde. Einige Teilnehmer nannten auch die Mast der Hähne der Legehennenlinien als eine mögliche Alternative zur Tötung von Eintagsküken, während die Verwendung von Zweinutzungshühnern überhaupt nicht erwähnt wurde. Als die Teilnehmer gefragt wurden, ob sie eine Idee hätten, was unter Zweinutzungshühnern zu verstehen sei, konnten sich nur wenige darunter etwas vorstellen. Die Teilnehmer antworteten zum Beispiel: "Ich habe keine Ahnung, was gemeint sein könnte. Haben die zwei Köpfe?" oder "Das klingt wie in der Roboterie hergestellt”. In Bezug auf den Begriff „Zweinutzungshuhn“ waren sich fast alle Teilnehmer darüber einig, dass die Benennung unangemessen sei und irreführende Vorstellungen verursache.

\subsection{Das Konzept Zweinutzungshuhn}

Da der Schwerpunkt unserer Untersuchung auf der Verbraucherperspektive liegt, wurde den Teilnehmern im Laufe der Diskussion das Konzept des
Zweinutzungshuhns erklärt. Die Reaktionen waren überwiegend positiv, aber es wurden auch Bedenken geäußert. Der am häufigsten genannte negative Aspekt war der höhere Preis für Fleisch und Eier von Zweinutzungshühnern. Die positiven Aspekte betrafen in erster Linie ethische Aspekte, da die männlichen Küken der Zweinutzungsrassen nicht als Eintagsküken getötet werden. Einige Teilnehmer vermuteten, dass die Fleischqualität aufgrund einer längeren Mastdauer und eines langsameren Fleischwachstums besser sein könnte. Andere empfanden die Situation als Dilemma zwischen dem Überleben der männlichen Küken und dem höheren Preis für Hühnerfleisch und Eier. Neben dem höheren Preis wurde von einem Diskutanten auch die ineffiziente Ressourcennutzung (u.a. aufgrund des erhöhten Futterbedarfs) der Zweinutzungshühner kritisch angemerkt. Ein weiterer Punkt, der von den Diskutanten betont wurde, betraf die Angst, dass Gentechnik für die Zucht von Zweinutzungshühnern verwendet werden könnte.

\subsection{Potenzielle Kaufkriterien}

Während der Diskussionen wurde deutlich, dass für viele Teilnehmer die alleinige Vermeidung des Kükentötens nicht ausreicht, um Produkte von Zweinutzungshühnern zu kaufen. Dies würden sie nur tun, wenn gleichzeitig auch die Haltungsbedingungen dieser Tiere verbessert werden. Als Beispiele für bessere Haltungsbedingungen wurden "gutes Futter", "keine Antibiotika", "viel mehr Platz" und "Einstreu" genannt. Auch die Kennzeichnung der Herkunft stellt für einige Diskutanten ein wichtiges Kaufkriterium dar. In diesem Zusammenhang wurde vorgeschlagen, die Adresse des Landwirtes auf der Verpackung anzugeben. Wie zu erwarten war, wurde auch der Preis als wichtiges Kaufkriterium genannt. Die Mehrheit der Diskussionsteilnehmer gab an, dass sie einen Aufpreis für Fleisch und Eier aus "Sympathie mit den Küken" oder um "Fleisch mit reinem Gewissen zu essen”, zahlen würden. Allerdings wurde diese Mehrzahlungsbereitschaft von einigen Diskutanten relativiert: "Es hängt davon ab, wie viel mehr ich bezahlen muss”. Im Fall von Eiern gaben einige Teilnehmer an, dass sie bereit seien, einen Aufpreis von bis zu $50 \%$ zu zahlen. Für Fleisch schien die Bereitschaft, einen Aufpreis zu zahlen, nicht so hoch zu sein, wie folgende Aussagen verdeutlichen: "Ich würde einen Aufpreis von $20 \%$ bezahlen, wenn das Fleisch besser schmeckt" und "ich würde nicht $10 €$ mehr bezahlen”. Mehr Geld für Fleisch auszugeben und gleichzeitig den Fleischverzehr zu reduzieren, 
wurde von mehreren Diskutanten als Lösung gesehen. Nur wenige Teilnehmer gaben an, dass sie nicht bereit seien, einen Aufpreis zu zahlen.

\section{Diskussion}

Die Ergebnisse dieser qualitativen Untersuchungen deuten an, dass es zu dem jetzigen Zeitpunkt schwierig werden könnte, die Produkte des Zweinutzungshuhns flächendeckend zu vermarkten. Die Eier der Hennen könnten den notwendigen Preis erzielen, da derzeit ein Preisaufschlag von zwei bis vier Cents pro Ei kalkuliert wird. Bei gleichzeitig verbesserter Haltung könnten sie durchaus in der Gunst der Konsumenten stehen. Für die männlichen Tiere sieht es dagegen problematisch aus. Neben dem, von den meisten Teilnehmern als zu hoch beschriebenen Preis $(7 €$ Preisaufschlag/kg Fleisch könnten laut Experten realistisch sein) kommt erschwerend hinzu, dass bisher keine Bereitschaft besteht, das eigene Kaufverhalten zu ändern.

Die Resultate aus den Gruppendiskussionen verdeutlichen, dass den Haltungsbedingungen eine herausragende Rolle im Hinblick auf die Mehrzahlungsbereitschaft der Verbraucher zukommt. Den meisten Diskussionsteilnehmern würde allein der Einsatz von Zweinutzungshühnern in einem sonst unveränderten Haltungssystem nicht genügen. Sie wünschen sich vorrangig eine verbesserte Haltung der Tiere. Auch wurde eine deutliche Kennzeichnung der geografischen Herkunft als potenzielles Kaufkriterium für Produkte von Zweinutzungshühnern hervorgehoben. Dies sind Aspekte, denen bei der Vermarktung von Produkten der Zweinutzungshühner Rechnung getragen werden sollte. Zudem zeigte sich, dass die Bezeichnung „Zweinutzungshuhn“ als ungeeignet und irreführend empfunden wurde, da sie in erster Linie negative Assoziationen hervorrief. Einige Diskussionsteilnehmer äußerten in diesem Zusammenhang Bedenken hinsichtlich des Einsatzes von Gentechnik beim Zweinutzungshuhn. Hier hat sich die Methodik der Gruppendiskussionen als sehr nützlich erwiesen, um Einblicke in die Bedenken der Verbraucher zu bekommen und um künftig möglichen Verwirrungen und Missverständnissen vorzubeugen. Diese Erkenntnisse hätten allein durch quantitative Erhebungsmethoden nicht erzielt werden können.

Den Teilnehmern wurde im Diskussionsverlauf eine Möglichkeit aufgezeigt, wie sie selbst Einfluss auf den Markt und damit auf die Verbreitung der Eintagskükentötung nehmen könnten. Sie bestand darin, sich vorzustellen, wie sie auf das Angebot von
Fleisch und Eiern von Zweinutzungshühnern reagieren würden. Dazu wurde ihnen mitgeteilt, dass diese Lebensmittel sich im Preis und in verschiedenen Eigenschaften von den derzeit marktüblichen Produkten unterscheiden würden. Das interessante Ergebnis besteht darin, dass die Verbraucher anscheinend nicht willens oder nicht in der Lage sind, eine rechtfertigungsfähige Auswahlentscheidung zwischen zwei jeweils unterschiedlich defizitären Produkten zu treffen. Sie forderten sehr deutlich Eier und Fleisch von Hühnern, deren Haltung keine Defizite aufweist. Nur unter dieser gedanklichen Prämisse waren die Teilnehmer bereit, über einen Aufpreis nachzudenken. Insgesamt fiel die Zustimmung zu dieser Option kleiner aus, als die Empörung zuvor erwarten ließ. Als Hauptgrund dafür wurden die übrigen vom Verbraucher bereits identifizierten Defizite in der Geflügelhaltung angeführt. Hier wurden insbesondere das Platzangebot pro Tier und fehlende Einstreu erwähnt. Dazu kamen aus der Sicht der Diskussionsteilnehmer die als problematisch wahrgenommene Behandlung der Tiere mit Antibiotika sowie Skepsis in Bezug auf die Futterqualität. Die Geflügelhaltung wurde als nicht transparent genug wahrgenommen.

In ethischer Hinsicht lieferten unsere Diskussionen mit Verbrauchern interessante Hinweise darauf, wie und wann Verletzungen des Moral- und Gerechtigkeitsempfindens $\mathrm{zu}$ einer Veränderung des Konsumverhaltens führen können. Die Diskussionen fanden zeitnah zur medialen Berichterstattung über das Gerichtsurteil zur Zulässigkeit der Eintagskükentötung statt. Darin kann einerseits ein glücklicher Zufall und andererseits eine Verzerrung der Untersuchung gesehen werden, da die Berichterstattung teilweise kritisch war. Bei den Diskussionsteilnehmern war die Empörung über die Praxis und das Urteil noch deutlich spürbar. Mehrheitlich wurde von ihnen die Praxis der Eintagskükentötung als nicht akzeptabel bewertet und ein gesetzliches Verbot gefordert.

Aufgrund der hier vorgestellten qualitativen Ergebnisse kann gegenwärtig damit gerechnet werden, dass sich Fleisch und Eier vom Zweinutzungshuhn nur als Nischenprodukt für bestimmte Käufersegmente eignen werden. Der nächste Schritt besteht darin, eine deutschlandweite Onlinebefragung mit 1500 Konsumenten von Hühnchenfleisch und Eiern auszuwerten und die Zahlungsbereitschaft für Eier und Fleisch von Zweinutzungshühnern zu ermitteln. Im SocialLab-Projekt werden diese Daten dann mit den Ergebnissen aus Interviews mit dem Lebensmitteleinzelhandel und 
Tierhaltern bezüglich möglicher Förder- und Hemmfaktoren im Hinblick auf die Haltung und Vermarktung von Zweinutzungshühnern und ihren Produkten zusammengeführt.

\section{Literatur}

Beckmann M (2016) Über den vernünftigen Grund im Sinne des §1 S. 2 TierSchG bei der Tötung von männlichen Eintagsküken. NuR 38(6):384-390

Bruijnis MRN, Blok V, Stassen EN, Gremmen HGJ (2015) Moral "Lock-In" in responsible innovation: the ethical and social aspects of killing day-old chicks and its alternatives. J Agric Environ Ethics 28(5):939-960

Damme K (2015) Economics of dual-purpose breeds a comparison of meat and egg production using dual purpose breeds versus conventional broiler and layer strains. LOHMANN Inf 50(2):4-9

Finch H, Lewis J (2003) Focus groups. In: Ritchie J, Lewis J (2003) Qualitative Research Practice, Sage Publications, London, pp 170-197

Flick U (2009) An introduction to qualitative research. Sage Publications, London

Grashorn M (2013) Verwendung der männlichen Küken der Legeherkünfte. http://www.wingvechta.de/ themen/verwendung_der_maennlichen_legeherk uenfte/verwendung_der_m_nnlichen_k_ken_der_ legeherk_nfte.html. Abgerufen 15.05.2016

Kaufmann F, Nehrenhaus U, Andersson R (2016) Das Duale Huhn. Der Verbraucher müsste umdenken. DGS 35:22-25

Koenig M, Hahn G, Damme K, Schmutz M (2012) Utilization of laying-type cockerels as „coquelets“: influence of genotype and diet characteristics on growth performance and carcass composition. Arch Geflugelkd 76(3):197-202

Kühn T, Koschel K (2011) Gruppendiskussionen. Ein Praxis-Handbuch. VS Verlag für Sozialwissenschaften, Wiesbaden

Lamnek S (2005) Gruppendiskussion. Theorie und Praxis. UTB, Weinheim

Leenstra FR, Munnichs G, Beekman V, Heuvel-Vromans E van den, Aramyan LH, Woelders H (2011) Killing day old chicks? Public opinion regarding potential alternatives. Animal Welf 20:37-45

Mayring P (2015) Qualitative Inhaltsanalyse. Grundlagen und Techniken. Beltz Verlag, Weinheim

Morgan DL (1997) Focus groups as qualitative research. Sage Publications, California

Rautenschlein S (2016) Einsatz des Zweinutzungshuhns in Mast und Eierproduktion: Ansätze für ein integriertes Haltungskonzept. Rundsch Fleischhygiene Lebensmittelüberwachung 68(8):276-278 


\section{Nutztierhaltung im Spiegel der Gesellschaft}

\section{Zusammenfassung und erste Schlussfolgerungen}

Inken Christoph-Schulz, Monika Hartmann², Peter Kenning ${ }^{3}$, Jörg Luy ${ }^{4}$, Marcus Mergenthaler ${ }^{5}$, Lucia Reisch $^{6}$, Jutta Roosen ${ }^{7}$, Achim Spiller ${ }^{8}$

${ }^{1}$ Thünen-Institut für Marktanalyse, Braunschweig

${ }^{2}$ Rheinische Friedrich-Wilhelm-Universität, Bonn

${ }^{3}$ Heinrich-Heine-Universität, Düsseldorf

${ }^{4}$ Privates Forschungs- und Beratungsinstitut für angewandte Ethik und Tierschutz INSTET gGmbH, Berlin

${ }^{5}$ Fachhochschule Südwestfalen, Soest

${ }^{6}$ Copenhagen Business School; Zeppelin Universität, Friedrichshafen

${ }^{7}$ Technische Universität München

${ }^{8}$ Georg-August-Universität, Göttingen

inken.christoph@thuenen.de

\section{Landwirte, Konsumenten ${ }^{14}$ und Bürger $^{15}$}

Wie Simons et al. in ihrem Beitrag zeigen, erfolgt bereits die Wahrnehmung der Nutztierhaltung in einem polarisierenden Wertungsrahmen. Die Wertungen sind implizit in den Bildern enthalten, die die Vorstellung der Studienteilnehmer zur Tierhaltung prägen und werden weniger oder gar nicht durch konkretes Wissen beeinflusst. Diese aus realen, medialen oder nur vorgestellten Erlebnissen resultierenden Bilder zeigen ein ausgeprägtes Spannungsfeld zwischen einer akzeptierten, schönen und ethisch unbedenklichen sowie einer nicht akzeptierten, unschönen und ethisch problematischen Nutztierhaltung. Insbesondere die Bilder einer idyllischen Welt, in der die Bauernfamilie mit den Tieren zusammen auf dem Hof im Rahmen einer kleinstrukturierten Landwirtschaft lebt, bilden den Maßstab für das, was als „gute Tierhaltung“ bezeichnet und dessen Umsetzung gewünscht wird. Landwirte und Tiere leben im Sinne eines „fairen Deals“ zusammen und sind aufeinander angewiesen. Dem Idealbild stehen Schreckensbilder gegenüber, die von den Studienteilnehmern von sich aus als „Massentierhaltung“

\footnotetext{
${ }_{14}$ Im Folgenden sind die Begriffe Konsument und Verbraucher als äquivalent anzusehen.

15 Im Folgenden wird sowohl von Konsumenten als auch von Bürgern gesprochen. Der Grund ist, dass in manchen Studien Konsumenten als Nachfrager auf Märkten, in anderen Studien Menschen in ihrer Rolle als Staatsbürger und „Verbraucherbürger“ (consumer citizen) untersucht wurden. Dieser unterschiedlichen Perspektive gilt es, Rechnung zu tragen.
}

bezeichnet werden. Diese dichotome Wahrnehmung der Nutztierhaltung wird in mehreren Beiträgen dieses Heftes angesprochen und detaillierter dargestellt. Sie prägt die individuelle und die öffentliche Wahrnehmung unabhängig vom Realitätsbezug und von der Realisierbarkeit der beschriebenen Bilder.

In Hinblick auf diese verbreiteten Bildmuster, die in vielen Bürgern tief verwurzelt zu sein scheinen, ist es nicht verwunderlich, dass sich zwischen Landwirten und Bürgern bzw. Verbrauchern teils große Unterschiede in der Wahrnehmung der Nutztierhaltung ergeben. Während viele Landwirte die in den vergangenen Jahrzehnten eingeführten Maßnahmen zur Effizienzsteigerung eher als erforderlich und daher als Verbesserung wahrnehmen, erkennen die befragten Bürger eine primär an einzelbetrieblicher Gewinnorientierung ausgerichtete Entwicklung. Für das einzelne Tier und seine natürliche Lebensweise wird diese Entwicklung als eher abträglich erachtet. Wie unterschiedlich dabei die Vorstellungen sind, zeigt sich am Beispiel der zunehmenden Technisierung in der Tierhaltung: Landwirte betonen die Vorzüge des hohen Technisierungsgrades, weil sie neben den damit verbundenen wirtschaftlichen Vorteilen und der Arbeitserleichterung mehr Zeit für die Tiere haben. Dagegen gehen die befragten Bürger davon aus, dass Landwirte aufgrund des hohen Technisierungsgrades seltener direkten Kontakt zu ihren Tieren haben. Allerdings halten es Landwirte und Bürger für notwendig, dass Landwirte Zeit mit ihren Tieren verbringen, bspw. um frühzeitig Krankheiten zu erkennen (Rovers et al.; Wildraut et al.; Wildraut und Mergenthaler).

Die Stallhaltung ist ein weiteres, kontrovers diskutiertes Thema. So betonen Landwirte, dass sie eher einen positiven Effekt der Stallhaltung hinsichtlich der Tiergesundheit sehen und daher der Weidehaltung bei Rindern bzw. der Freilandhaltung bei Schweinen und Hühnern kritisch gegenüberstehen (Wildraut und Mergenthaler). Die Verbraucher und Bürger sind gegenteiliger Auffassung (Kühl et al.; Rovers et al.) und lehnen eine reine Stallhaltung fast immer ab. Die Weide- bzw. Freilandhaltung wird präferiert. Dabei ist die Vermutung, dass die Tiere dadurch ihr natürliches Verhalten ausleben können, ein Hauptargument. Allerdings kann bereits die Gewährung einer Auslaufmöglichkeit die Akzeptanz erhöhen (Kühl et al.).

Wie relevant Haltungsaspekte der Tiere für die Zahlungsbereitschaft sind, zeigen Brümmer et al. und Roosen et al. Bei Betrachtung unterschiedlicher Maßnahmen in der Legehennen- und Masthühnchenhaltung wird deutlich, dass die Befragten das 
Töten der männlichen Eintagsküken in der Legehennenhaltung ablehnen. Als Lösungsansatz werden von den Befragten beider Studien die Züchtung von Zweinutzungsrassen und die Geschlechtsbestimmung im Ei befürwortet. Die von Brümmer et al. untersuchten Konsumenten machten außerdem deutlich, dass eine Mehrzahlungsbereitschaft daran gebunden ist, nicht nur die Eintagskükentötung zu vermeiden, sondern auch die Haltungsbedingungen zu verbessern. Roosen et al. kommen zu einem ähnlichen Ergebnis. Beide Studien beruhen auf hypothetischen Entscheidungen der Befragten. Allerdings lassen sich hieran die Prioritäten der Bürger und Verbraucher ablesen, mit denen in ihren Augen kritische Aspekte der Tierhaltung zu ändern sind.

\section{Verbraucherkommunikation und - informationen}

Sonntag et al. zeigen in ihren Untersuchungen zur Bewertung von Zielkonflikten, dass zusätzliche Informationen über die Tierhaltung eine besonders durchdachte Vorgehensweise benötigen. Trotz neutraler Informationen durch Bilder und Texte verbessert sich die Wahrnehmung von als negativ empfundenen Haltungssystemen nicht. Außerdem führt die Konfrontation der Studienteilnehmer mit verschiedenen Nachteilen der von ihnen als positiv empfundenen Auslaufhaltung nicht dazu, dass sie diese Haltungsform kritischer sehen. Seitens der Verbraucher herrscht zudem größtenteils Übereinstimmung, dass es Aufgabe der Landwirte ist, existierende Zielkonflikte zu lösen. Dies sollte mit Hilfe von Innovationen erfolgen. Zudem wird deutlich, dass sich die befragten Verbraucher im Falle eines Zielkonfliktes zwischen Tierwohl und Umweltschutz bzw. zwischen Tierverhaltensoptionen und Tiergesundheit für ein Mehr an Tierwohl und für Natürlichkeit entscheiden.

Gier, Krampe et al. untersuchen die Wirkung verschiedener Kommunikationsmaßnahmen auf die Verbraucherwahrnehmung. Sie kommen zu dem Ergebnis, dass unterschiedliche Darstellungsweisen der Tierhaltung eine spezifische neuropsychologische Wirkung entfalten, die sich in unterschiedlichen expliziten Bewertungen der kommunizierten Maßnahmen manifestiert. Die Autoren folgern, dass es grundsätzlich möglich erscheint, eine die subjektiven, impliziten Wertungen beeinflussende, akzeptanzfördernde Kommunikationsstrategie zu entwickeln. Auf Basis ihrer Ergebnisse plädieren sie für entsprechend gestaltete
„Verbraucherinformationssysteme“, die Konsumenten unter Berücksichtigung neuropsychologischer Erkenntnisse individuell nach situativer und persönlicher Relevanz und Involvement über Produkte informieren, um ihnen eine bewusste auf ihre Bedürfnisse zugeschnittene Kaufentscheidung zu ermöglichen. In Hinblick auf Verbraucherinformationen und ihre Auswirkungen auf befragte Verbraucher analysieren Groß und Roosen den Einfluss negativ ${ }^{16}$ und positiv ${ }^{17}$ formulierter Nachrichtentexte auf das Vertrauen. So haben negative Nachrichten generell einen größeren Einfluss auf das Verbrauchervertrauen als positive.

Gier, Krampe et al. zeigen außerdem, wie bedeutsam die implizite Wirkungsweise von Darstellungsvarianten der Tierhaltungsverfahren ist. Demzufolge kann eine Information, welche sich auf die Errungenschaften sowie Erfolge einer Tierhaltungsmaßnahme bezieht, implizit die Bewertung dieser beeinflussen. Des Weiteren spielt das Entscheidungsumfeld eine wesentliche Rolle, denn Bildkommunikationsmaßnahmen über die Nutztierhaltung haben offenbar nur im direkten Entscheidungskontext, z.B. am Point-of-Sale, einen Einfluss auf die implizite und subjektive Wahrnehmung bzw. Bewertung der Information.

\section{Handel}

Krampe et al. betrachten in ihrem Beitrag den Handel als Gatekeeper zwischen Lieferant und Verbraucher. Sie beleuchten drei Schlüsselaspekte: die Listungsentscheidung, die Sortimentsvielfalt sowie die Regulierung des Marktes. Aus den Untersuchungen geht hervor, dass in Bezug auf die Listungsentscheidung versucht wird, durch standardisierte Prüfungen der Lieferanten, Verbrauchern einen vertrauensvollen Fleischkauf und -konsum zu gewährleisten. Dabei wird offenbar vor allem auf langfristige Partnerschaften gesetzt. Hinsichtlich der Sortimentsvielfalt und Produktauswahl wird das Kaufverhalten der Verbraucher als entscheidend beschrieben. Ein Versuch als „moralischer Wahlhelfer $^{“}$ zu agieren, erfolgt dagegen nicht. Bezüglich möglicher Regulierungen werden zwar durchaus Vorteile gesehen, allerdings wird der Markt als ein grundsätzlich effektives System betrachtet, das eigentlich keiner weiteren Regulierung bedarf. Label,

\footnotetext{
$\overline{16}$ Beispiel negative Information: Tierhaltung muss sich verbessern.

17 Beispiel positive Information: Erzielen von Fortschritten im Sachen Tierhaltung.
} 
seien sie staatlich oder privatwirtschaftlich getragen, werden eher kritisch gesehen. Hier wird die Gefahr einer Überlastung der Verbraucher mit zusätzlichen Informationen beschrieben, die das eigentliche Ziel, die Verbraucher beim Einkauf $\mathrm{zu}$ unterstützen, verfehlt.

\section{Erste Schlussfolgerung und Perspektiven}

Aus den vorliegenden Arbeiten können die folgenden ersten Schlussfolgerungen gezogen werden. $\mathrm{Zu}$ beachten ist, dass aus den Werthaltungen, Einstellungen und Präferenzen der verschiedenen, im Projekt untersuchten, gesellschaftlichen Gruppen (Bürger, Konsumenten, Landwirte, Händler) keine direkten Handlungsempfehlungen abgeleitet werden können. Die Ausgestaltung einer zukunftsfähigen Tierhaltung ist vielmehr das Ergebnis eines gesellschaftlichen Diskurses, in den neben den verschiedenen gesellschaftlichen Positionen auch naturwissenschaftlich-technische sowie verbraucherwissenschaftliche Aspekte einfließen. Der innovative Charakter von SocialLab liegt darin, in systematischer Form Politik, Zivilgesellschaft und Wirtschaft über die Positionen der Beteiligten aufzuklären und den notwendigen Diskurs damit transparenter und informationshaltiger zu gestalten.

\section{Landwirte}

In offenen Märkten können landwirtschaftliche Familienbetriebe und Unternehmen nur bestehen, wenn sie wettbewerbsfähig sind. Deshalb können sie nicht losgelöst von wirtschaftlichen und politischen Rahmenbedingungen agieren. Dies gilt folglich auch in Hinblick auf Innovationen, die ein höheres Maß an Tierwohl gewährleisten. Im Rahmen der Diskussion um Verbesserungen in der Tierhaltung ist es außerdem entscheidend, wie schnell verschiedene Maßnahmen umgesetzt werden können. Die befragten Landwirte betonen, dass neue Stallbaukonzepte nicht kurzfristig umzusetzen sind, da Stallbauten auf lange Abschreibungsfristen ausgelegt sind und sich als Investition mit hoher Spezifität und wenig Nutzungsalternativen ausweisen. Mehr Möglichkeiten werden bei der spezifischen Ausgestaltung der Ställe gesehen. Zu nennen sind in diesem Zusammenhang u.a. die Implementierung verschiedener Funktionsbereiche (z.B. Liege-, Fress- Kot-, Aktivitätsbereich), um den Tieren ein Mehr an artgerechtem Leben zu ermöglichen. Auch die Einführung bautechnisch flexiblerer Systeme, wie z.B. flexible Liegeboxen und Freiflächen zum Liegen in der Milchviehhaltung oder flexible Kastenstände in der Sauenhaltung, um tierindividuelle Größenunterschiede zu berücksichtigen, kann helfen, vorherrschende Anforderungen von Konsumenten und Bürgern umzusetzen. Zudem sollte auf Beschäftigungsmöglichkeiten geachtet werden die in bereits bestehende Stallsysteme integriert werden können. Außerdem wird die stärkere Betonung der Zuchtziele Tiergesundheit und Robustheit als ein wichtiger, von Stallbausystemen losgelöster Aspekt für ein Mehr an Tierwohl gesehen.

Aus Sicht der Landwirte wären technisch flexible Systeme und langfristig verlässliche ökonomische und politische Rahmenbedingungen auf der einen Seite und weniger ordnungspolitische Vorgaben auf der anderen Seite wünschenswert. Begründet wird dies neben dem Schutz der unternehmerischen Freiheit auch damit, dass Tierwohl stärker vom Tier her und ergebnisorientiert betrachtet werden sollte. Landwirte sehen darin die Voraussetzung, um in offenen Märkten und im Wettbewerb mit dem Lebensmitteleinzelhandel die Zukunftsfähigkeit der eigenen Familienbetriebe zu sichern. Dabei ist auch zu bedenken, dass die Einführung eines Mehr an Tierwohl auf Seiten der Landwirte eine größere Chance haben wird, wenn die Veränderungen im Einklang mit der Wahrnehmung, den Werten und dem beruflichen Selbstverständnis der Betriebsleiter sind und die Aussicht gegeben ist, durch die Veränderungen brancheninterne Anerkennung und gesellschaftliche Wertschätzung zu erfahren. Die Weiterentwicklung der Rahmenbedingungen für mehr Tierwohl sollten also zum einen persönliche Erfolge der Betriebsleiter und zum anderen finanzielle Erfolge der veränderungsbereiten Betriebe ermöglichen. Einflussfaktoren auf die einzelbetrieblichen Entscheidungen zur Übernahme und Weiterentwicklung technischer und organisatorischer Innovationen für mehr Tierwohl sollten hierbei stärker in den Fokus zukünftiger Forschung rücken. In diesem Zusammenhang sollten explizit auch verhaltensökonomische Untersuchungsdesigns Anwendung finden, die praxisnah Wirkzusammenhänge erforschen. Darauf basierend lassen sich spezifische Informationen für politische Entscheidungen ableiten, die eine Weiterentwicklung zu einer in der Breite akzeptierten Tierhaltung ermöglichen.

\section{Bürger bzw. Verbraucher}

Zusätzlich zu den Bedürfnissen der Landwirte ist die Berücksichtigung gesellschaftlicher Ansprüche und Erwartungen von hoher Bedeutung. Bürger entscheiden in ihrer Doppelrolle als Verbraucher und als Wähler langfristig mit, was am Markt existieren kann und was nicht (Allen et al. 1991; Buller und Roe 2012; 
Weary et al. 2016). Die bisher vorliegenden Arbeiten des SocialLab zeigen, dass sich Studienteilnehmer mitunter gar nicht mit der Thematik ,Tierwohl in der Nutztierhaltung“ beschäftigen möchten. Andere wiederum beschreiben ihre Vorstellungen dagegen ausführlich und formulieren umfangreiche Erwartungen. Die Vorstellungen sind dabei häufig durch die mediale Berichterstattung geprägt und nur in seltenen Fällen liegen spezifische Kenntnisse über die Tierhaltung vor.

In den meisten Fällen fühlen sich die befragten Teilnehmer in ihrer Rolle als Konsument überfordert. Einerseits akzeptieren sie das, was sie über die Nutztierhaltung zu wissen glauben, nicht, andererseits tolerieren viele von ihnen die Zustände in ihrem alltäglichen Einkaufsverhalten. Die Gründe für dieses Verhalten sind vielschichtig. Generell gilt jedoch zu berücksichtigen, dass der Lebensmitteleinkauf nicht die einzige Herausforderung im Alltag der Verbraucher ist. Werden Bürger bzw. Verbraucher explizit mit Fragen der Nutztierhaltung konfrontiert, zeigen sie sich zumeist auch dann ausgesprochen kritisch, wenn sie sich zuvor nicht mit dem Thema beschäftigt haben. Zahlungsbereitschaftsstudien ermitteln immer wieder eine Mehrzahlungsbereitschaft für Tierwohlaspekte und es wird auch zukünftig wichtig sein, die Präferenzstruktur zu kennen und bei der Verbraucherkommunikation zu berücksichtigen. Diskrepanzen zwischen am Point-of-Sale gezeigten und in Befragungen geäußerten Zahlungsbereitschaften können aber auch ein Hinweis auf eine Trittbrettfahrer-Problematik sein, wie sie bei der Bereitstellung öffentlicher Güter auftritt. Hier wäre zu klären, wie ein mögliches Marktversagen durch politische Eingriffe oder organisatorisch-institutionelle Innovationen behoben werden kann und Verbrauchern Konsumentscheidungen ermöglicht werden können, die in Einklang mit ihren Präferenzen stehen.

\section{Kommunikation}

Für eine Verbesserung der Akzeptanz der Tierhaltung sind Änderungen und Weiterentwicklungen hin zu tiergerechteren Verfahren notwendig, aber nicht hinreichend. Die hier vorliegenden Ergebnisse zeigen, dass Verbesserungen in der Tierhaltung Bürgern und Verbrauchern gegenüber unter Berücksichtigung (neuro-)psychologischer und verhaltensökonomischer Zusammenhänge kommuniziert werden müssen. Hierbei kommt der Kommunikation eine Sonderstellung zu. Es ist dabei notwendig, die Empfindungskategorien Mitgefühl, Gerechtigkeit und Respekt beim Bürger/Verbraucher anzusprechen, da mit größerer Akzeptanz durch den Bürger bzw. Verbraucher zu rechnen ist, wenn der Eindruck eines „fairen Deals“ zwischen Mensch und Tier entsteht (Nutzung tierischer Produkte gegen gutes Leben des Tieres). Kommunikation ist somit ein entscheidender Bestimmungsfaktor für die Entwicklung von Akzeptanz. Dabei scheint der oftmals impliziten Wirkungsweise von Darstellungsvarianten der Tierhaltungsverfahren eine besondere Bedeutung zuzukommen. Im Rahmen der bisher vorliegenden Arbeiten des SocialLab spielt der Präsentationsrahmen - das sogenannte Framing - eine entscheidende Rolle in der Kommunikationswahrnehmung und -verarbeitung der Verbraucher. Es ist daher wichtig, diese Aspekte bei der Gestaltung von Kommunikation im Bereich der Nutztierhaltung künftig noch stärker zu beachten. Das „Wie?“ der Gestaltung würde dann stärker betont werden müssen. Gleichwohl gilt es zu vermeiden, dass entsprechende Maßnahmen als schönfärbend wahrgenommen werden, um einen möglichen weiteren Glaubwürdigkeitsverlust des Sektors zu vermeiden.

In Hinblick auf eine zielgerichtete Verbraucherinformation wären unter Berücksichtigung informationslogistischer Erkenntnisse u.a. folgende Maßnahmen denkbar: stärkere Nutzung sozialer Netzwerke, die bereits heute in vielen Branchen genutzt werden. Sie könnten ein flexibles Element eines modernen „Verbraucherinformationssystems“ bilden, welches auch in Hinblick auf die gesellschaftliche Akzeptanz der landwirtschaftlichen Nutztierhaltung wünschenswert wäre. Mit Hilfe einer Moderation und Experten (Landwirte, Händler und Wissenschaftler) können verifizierte Nutzer (Verbraucher) über für sie relevante Themen im Bedarfsfalle untereinander und mit unabhängigen Experten diskutieren und Erfahrungen austauschen. Dadurch könnten relevante Informationen ohne (große) Überforderung den interessierten Verbrauchern zur Verfügung gestellt werden. Der Informationsgegenstand könnte dabei gezielt durch den Verbraucher erbeten werden, aber zugleich auch aktiv von den Experten vermittelt werden. Die aktuell an vielen Stellen beobachtbaren Informationsineffizienzen könnten so reduziert werden. Durch das Gewährleisten der Unabhängigkeit der Inhalte und eines glaubwürdigen Monitorings würde Vertrauen in das Verbraucherinformationssystem aufgebaut. Die verwendeten Daten sollten von öffentlichen Institutionen wie z.B. der Bundesanstalt für Landwirtschaft und Ernährung (BLE) verwaltet werden. Auch wenn Label mitunter als die einfachste Möglichkeit der Verbraucherinformation angesehen 
werden, stoßen sie regelmäßig an ihre Grenzen. Bedarfsgerechte, differenziertere, horizontal und vertikal organisierte Alternativansätze könnten zumindest für einige Verbrauchersegmente zu einer Lösung beitragen. Dabei ist es erforderlich, die Heterogenität der Verbraucher besser als bisher abzudecken, ihren Informationsbedarf flexibel $\mathrm{zu}$ bedienen und so einer Überforderung entgegenzuwirken.

\section{Handel}

Bei Listungsentscheidungen für Produkte mit einem höheren Tierwohlstandard setzen der Lebensmitteleinzelhandel (LEH) und weitere handelsnahe Akteure vor allem auf standardisierte Prozesse und langfristige Partnerschaften. Oft werden relativ einfache Heuristiken benutzt, die wissenschaftlich noch wenig durchdrungen sind. Durch eine Aufklärung ihres Gebrauchs könnte die Qualität der Listungsentscheidung von Tierwohlprodukten verbessert werden. Des Weiteren werden Label kritisch vom LEH und weiteren handelsnahen Akteuren betrachtet. Hier wird die Gefahr einer Überlastung der Verbraucher mit zusätzlichen Informationen erkannt, die das eigentliche Ziel der Verbrauchererleichterung beim Einkauf verfehlt. Um die Überlastung der Verbraucher zu verringern, sollten weitere Anreize für den LEH zur Beteiligung an der bereits genannten systemischen Lösung (Gier et al.) geschaffen werden. Förderlich wären hier auch Anreize, die eine weiterführende Entwicklung entsprechender Marktbzw. Marketinginnovationen unterstützen (z.B. im Hinblick auf handels- und steuerrechtliche Aspekte).

\section{Summa summarum}

Die Nutztierhaltung hat in den vergangenen Jahrzehnten an gesellschaftlicher Akzeptanz verloren. Um diese Akzeptanz zurückzugewinnen, wird es notwendig sein, Veränderungen herbeizuführen, die vom Laien (Verbraucher, Bürger) als Verbesserung wahrgenommen werden. Die SocialLab-Zwischenergebnisse deuten allerdings darauf hin, dass Landwirte und Laien die Nutztierhaltung unterschiedlich wahrnehmen. Für zukünftige Forschungsarbeiten bezüglich innovativer Haltungssysteme ist es daher erforderlich, diese Arbeiten in einem großen disziplinübergreifenden Verbund durchzuführen, um die Thematik ganzheitlich zu untersuchen und existierende Problemfelder zu lösen bzw. neu entstehende frühzeitig zu entdecken. Im besten Falle würden Prototypen und innovative Ansätze für bspw. neue Stallsysteme entwickelt werden, die anschließend aus unterschiedlichsten
Blickwinkeln beurteilt und bei Bedarf angepasst und weiterentwickelt werden. Dabei muss bei Tierwohlaspekten auch eine betriebswirtschaftliche Betrachtung unter Berücksichtigung ökonomischer, politischer und gesellschaftlichen Rahmenbedingungen erfolgen. Landwirte und berufsständische Vertretungen müssen frühzeitig eingebunden werden, um die Umsetzbarkeit, auch unter Berücksichtigung von Aspekten wie der Arbeitssicherheit, zu klären. Parallel bedarf es der frühzeitigen Untersuchung der Wahrnehmung und Akzeptanz innovativer Ansätze durch die Gesellschaft, um zu vermeiden, dass aufgrund von Fehlinterpretationen etwaige Innovationen von vornherein abgelehnt werden. Wie oben dargestellt, geben die Verbraucher im Falle von Zielkonflikten zwischen Tierwohl und bspw. Umweltschutz dem Tierwohl Vorrang. Und auch aus der Nationalen Nutztierstrategie (BMEL 2017) geht hervor, dass bei Unvereinbarkeiten bspw. hinsichtlich des Umweltschutzes, dem Tierwohl Vorrang zu geben ist.

Ein derart multidisziplinärer Ansatz entspricht zum einen dem Innovationscharakter des SocialLab, das sich auch zum Ziel gesetzt hat, Innovationen, wie beispielsweise neue Formen der Tierhaltung oder züchterische Veränderungen aus der Perspektive unterschiedlicher gesellschaftlicher Gruppen zu untersuchen. Zum anderen deckt er sich mit Plänen des BMEL in Bezug auf die Entwicklung eines „Stalls der Zukunft“ (BMEL 2017).

Das BMEL fördert mit SocialLab einen ungewöhnlich breiten und methodisch vielfältigen Forschungsverbund. Die in diesem Heft vorgestellten Zwischenergebnisse bieten facettenreiche Informationen zum Status quo. Der Transformationsprozess in der Tierhaltung wird allerdings ein langfristiger Weg werden. Entwürfe für den „Stall der Zukunft“ werden in den nächsten Jahren im Hinblick auf ihre Wahrnehmung und gesellschaftliche Akzeptanz zu prüfen sein. Hierfür bietet SocialLab neben der Entwicklung innovativer Ansätze auch die Chance, die vorliegenden ersten Evidenzen zukünftig in Form einer Längsschnittstudie und begleitet durch ad-hocStudien zu zentralen Einzelfragen systematisch weiterzuführen und $\mathrm{zu}$ einem wissenschaftlichen Monitoring des Transformationsprozesses auszubauen. 


\section{Literatur}

Allen P, van Dusen D, Lundy J, Gliessmann S (1991) Integrating social, environmental, and economic issues in sustainable agriculture. Am J Altern Agric 6:34-39

BMEL (2017) Nutztierhaltungsstrategie. Zukunftsfähige Tierhaltung in Deutschland. http://www. bmel.de/DE/Tier/_texte/Nutztierhaltungsstrategie. html Abgerufen 19.10.2017

Buller H, Roe E (2012) Modifying and commodifying farm animal welfare: the economisation of layer chickens. J Rural Stud 33:141-149
Porter ME, Kramer MR (2011) Creating shared value. Harvard Bus Rev Jan./Febr, 63-70

Weary DM, Ventura BA, von Keyserlingk MAG (2016) Societal views and animal welfare science: understanding why the modified cage may fail and other stories. Animal 10(2):309-317

Open Access This article is distributed under the terms of the Creative Commons Attribution 4.0 International License (http://creativecommons.org/licenses/by/4.0/), which permits unrestricted use, distribution, and reproduction in any medium, provided you give appropriate credit to the original author(s) and the source, provide a link to the Creative Commons license, and indicate if changes were made. 